\title{
Systematic comparison of the molecular mechanisms underlying the spreading of pathology in different neurodegenerative diseases
}

\author{
Dissertation \\ for the award of the degree \\ "Doctor rerum naturalium" (Dr. rer. nat.) \\ of the Georg-August-Universität Göttingen, \\ Faculty of Biology
}

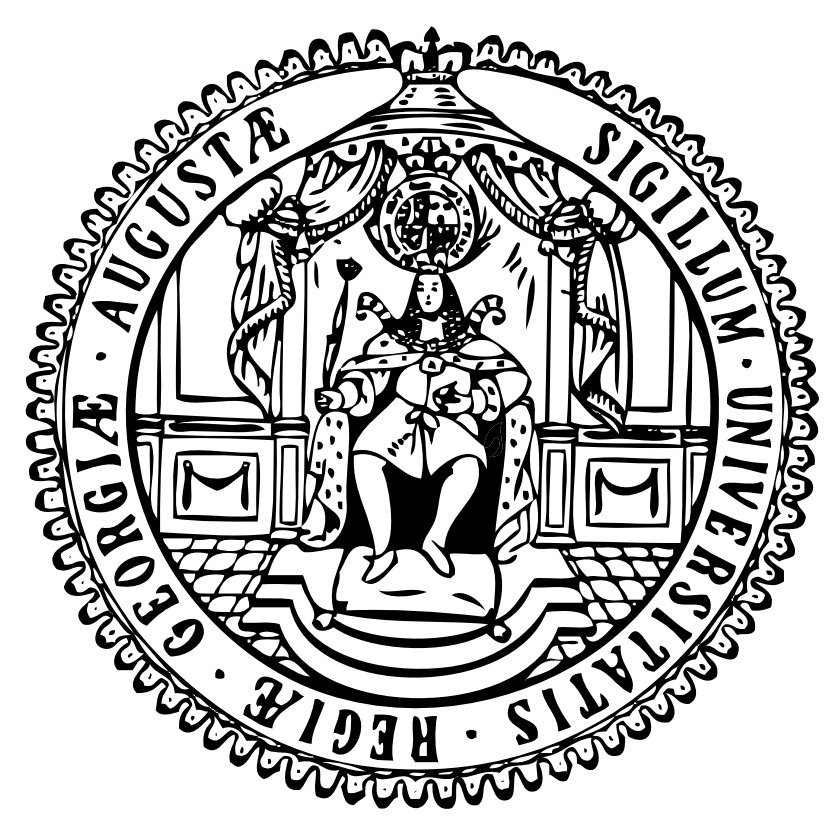

within the doctoral program Molecular Physiology of the Brain

of the Georg-August University School of Science (GAUSS)

submitted by

Inês Caldeira Brás

from Figueira da Foz, Portugal

Göttingen 2021 



\section{Thesis Committee}

Prof. Dr. Tiago Outeiro (1 ${ }^{\text {st }}$ Reviewer)

Dept. of Experimental Neurodegeneration, University Medical Center Göttingen, Göttingen.

Prof. Dr. Gerhard Braus (2 ${ }^{\text {nd }}$ Reviewer)

Dept. of Molecular Microbiology and Genetics, University Medical Center Göttingen, Göttingen.

Prof. Dr. Markus Zweckstetter

German Center for Neurodegenerative Diseases (DZNE), Göttingen

\section{Extended Thesis Committee Members}

Prof. Dr. Nils Brose

Dept. of Molecular Neurobiology, Max Planck Institute for Experimental Medicine, Göttingen.

Prof. Dr. Silvio Rizzoli

Department of Neuro- and Sensory Physiology, University Medical Center Göttingen, Göttingen.

Prof. Dr. Henning Urlaub

Bioanalytical Mass Spectrometry group, Max Planck Institute for Biophysical Chemistry, Göttingen.

Date of oral examination: October $15^{\text {th }}, 2021$ 


\section{List of publications integrated in the thesis}

1. Inês Caldeira Brás, Mohammad Hossein Khani, Dietmar Riedel, Iwan Parfentev, Ellen Gerhardt, Christoph van Riesen, Henning Urlaub, Tim Gollisch, Tiago Fleming Outeiro. (2021) Ectosomes and exosomes are distinct proteomic entities that modulate spontaneous activity in neuronal cells. BioRxiv. doi: 10.1101/2021.06.24.449731. In revision.

2. Inês Caldeira Brás, Mohammad Hossein Khani, Eftychia Vasili, Wiebke Möbius, Dietmar Riedel, Iwan Parfentev, Ellen Gerhardt, Christiane Fahlbusch, Henning Urlaub, Markus Zweckstetter, Tim Gollisch, Tiago F. Outeiro. (2021) Common molecular mechanisms underlie the transfer of alpha-synuclein, Tau and huntingtin and modulate spontaneous activity in neuronal cells. BioRxiv doi: 10.1101/2021.07.18.452825. In revision.

The work included in this thesis was conducted in the Department of Experimental Neurodegeneration, Georg-August-Universität Göttingen, in the group of Prof. Dr. Tiago Outeiro. 


\section{Acknowledgements}

"With work and determination, you can accomplish all that you want, the important is to have health to fight for it"

Graça Brás

First and foremost, I would like to express my sincere gratitude to my supervisor Prof. Dr. Tiago Fleming Outeiro for his guidance and support during my doctoral degree, and the opportunity given to be part of his group. It was a pleasure and a rewarding journey for my desire to become an excellent scientist.

To Prof. Dr. Gerhard Braus and Prof. Dr. Markus Zweckstetter, thank you for the positive discussions and comments during the thesis committee meetings, and time dedicated to this work. I would also like to thank all the collaborators that contributed for the development of this project.

To the SynDegen consortium coordinators and members, I want to express my gratefulness for the opportunity to be part of such an excellent group of scientists, with whom I had a great pleasure to meet and work. A special thank you for the principal investigators and respective groups that received me during the secondments, giving me the opportunity to learn beyond my field.

To the colleagues in the Department of Experimental Neurodegeneration, thank you for the insightful comments, encouragement and help during the development of my research.

Finally, a very special thanks to my friends and family, without whose motivation, immense knowledge, and precious support it would not be possible to conduct this research and reach this moment.

After all, it is the spark of science that make us build our own path in the scientific world. 


\section{Table of Contents}

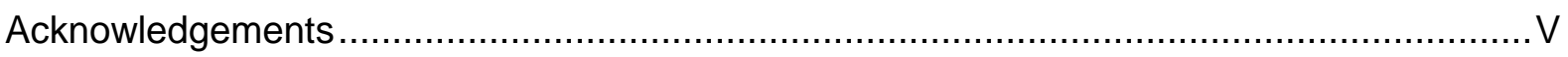

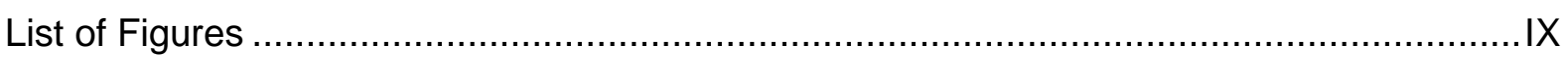

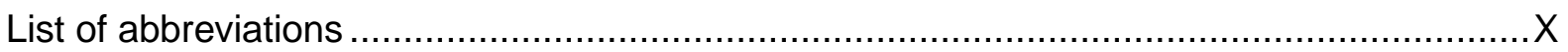

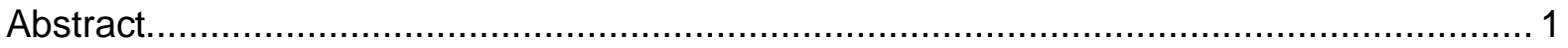

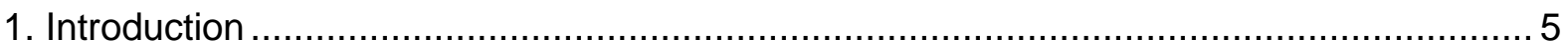

1.1. Alpha-synuclein in Parkinson's disease and other synucleinopathies ...................... 6

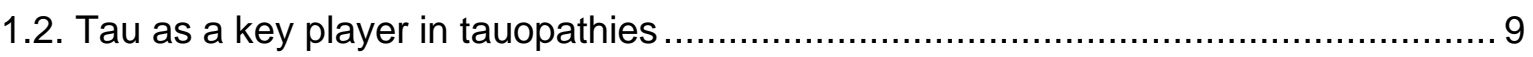

1.3. Polyglutamine repeats in Huntington's disease and other monogenic

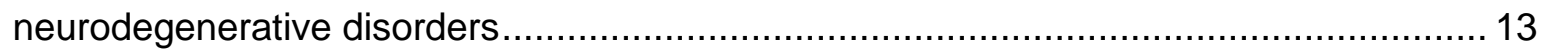

1.4. Prion diseases, protein misfolding disorders and prionoids ................................. 16

1.5. The role of prion-like spreading mechanisms in the progression of neurodegenerative diseases.

1.5.1. Extracellular vesicles as relevant mediators in intercellular communication in physiological and pathological conditions ....................................................... 21

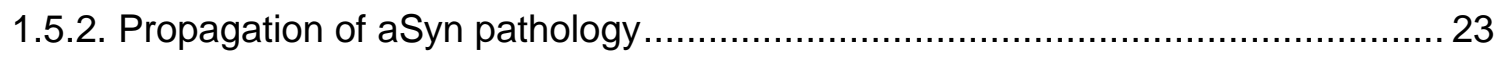

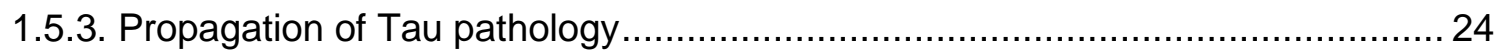

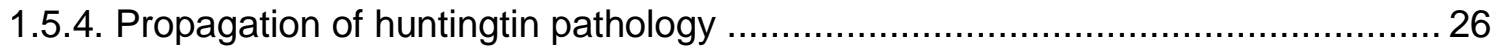

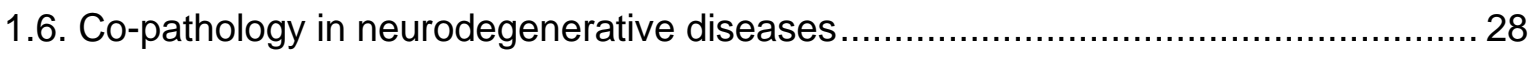

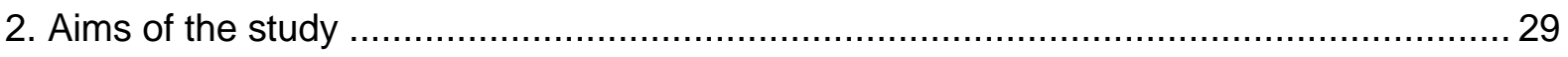

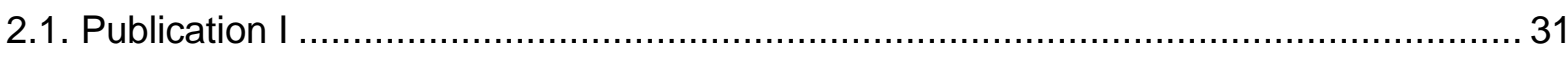

Ectosomes and exosomes are distinct proteomic entities that modulate spontaneous

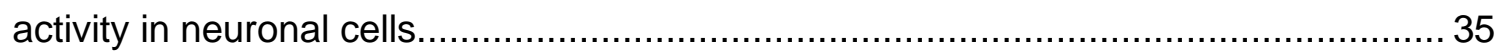

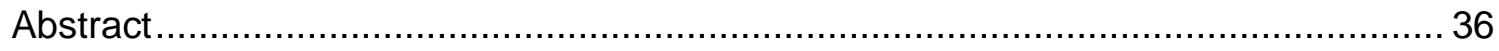

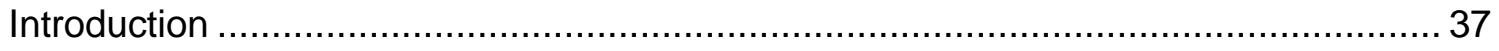

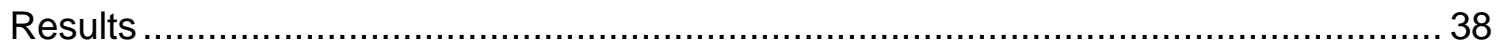

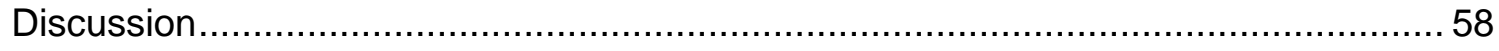

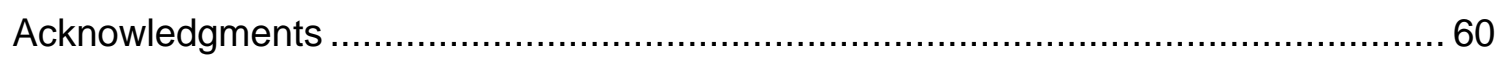

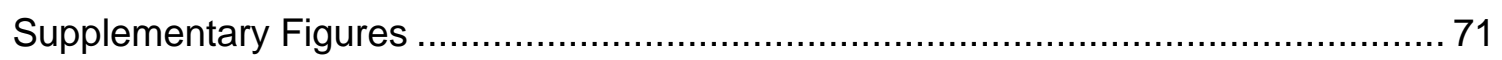

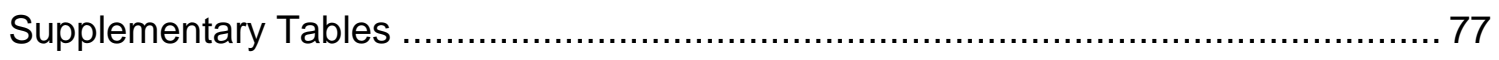

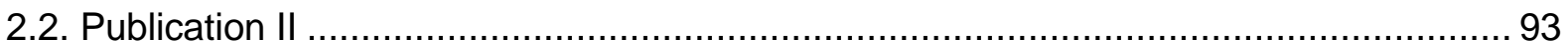


Common molecular mechanisms underlie the transfer of alpha-synuclein, Tau and huntingtin and modulate spontaneous activity in neuronal cells. 97

Abstract. 98

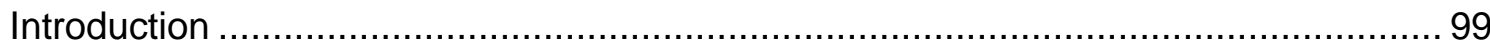

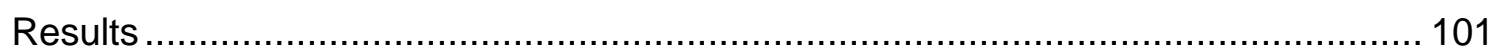

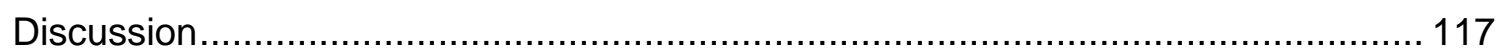

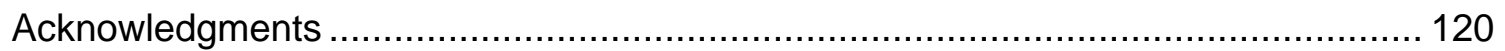

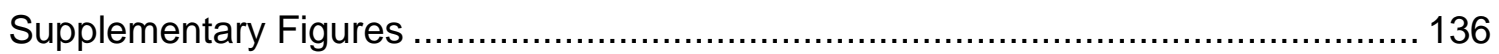

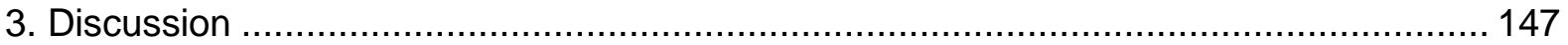

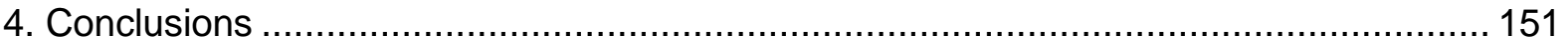

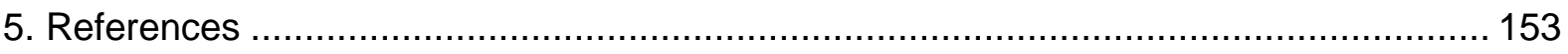

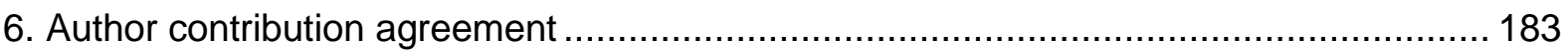

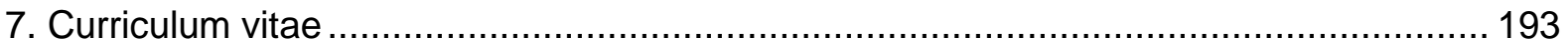




\section{List of Figures}

Figure 1. Schematic representation of aSyn structural domains and familial mutations........ 7

Figure 2. Several pathways are affected by aSyn misfolding and aggregation in

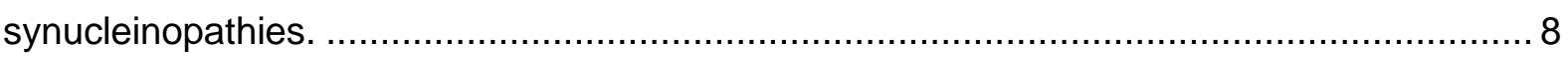

Figure 3. Physiological and pathological functions of human Tau. .................................. 10

Figure 4. Cellular mechanisms involved in the pathogenicity of mutant huntingtin (HTT) in

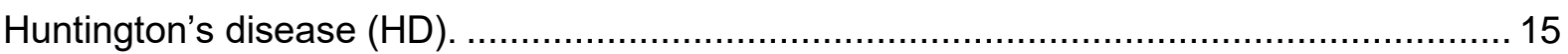

Figure 5. Proposed templated misfolding of disease-related proteins in neurodegenerative

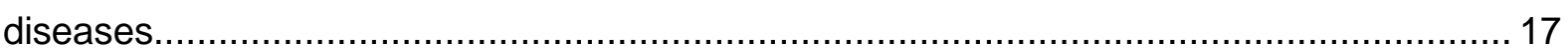

Figure 6. Protein misfolding disorders, prionoids and prions. ....................................... 18

Figure 7. Proposed mechanisms involved with transmission of disease-related proteins

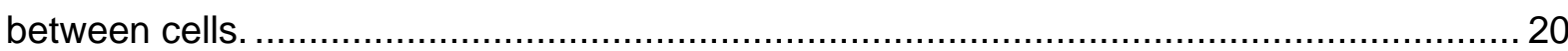

Figure 8. Exosomes and microvesicles transport numerous cargoes, as lipids, proteins and nucleic acids. 21 


\section{List of abbreviations}

Alpha-synuclein- aSyn

Alzheimer's disease- AD

Beta-synuclein- bSyn

Cerebrospinal fluid- CSF

Central nervous system- CNS

Endoplasmic reticulum- ER

Enteric nervous system- ENS

Extracellular vesicles- EVs

Frontotemporal dementia with parkinsonism linked to chromosome 17- FTDP-17

Frontotemporal lobar degeneration- FTLD

Gamma-synuclein- gSyn

Glycosylphosphatidylinositol- GPI

Heparan sulphate proteoglycans- HSPGs

Huntingtin- $\mathrm{Htt}$

Huntington's disease- HD

Lewy bodies- LBs

Lewy neurites- LNs

Microtubule-associated protein- MAP

Misfolding-associated protein secretion pathway- MAPS

Multivesicular bodies- MVBs

Mutant huntingtin- $\mathrm{mHtt}$

Neurofibrillary tangles- NFTs

Non-amyloid $\beta$ component- NAC

Nuclear export signal- NES

Nuclear localization signal- NLS

Parkinson's disease- PD 
Polyglutamine-polyQ

Post-translational modifications- PTMs

Prion protein- $\operatorname{PrPC}$

Proline-rich domain- PRD

Scrapie prion protein- PrPSc

Serine 129- S129

Transmissible spongiform encephalopathies- TSE

Tunnelling nanotubes- TNTs 



\section{Abstract}

Misfolding and accumulation of disease-related proteins are common hallmarks among several neurodegenerative diseases. This phenomenon causes the progressive loss of cognitive and motor functions correlated with specific cell loss in the brain. Despite the different clinical manifestations, these disorders share common features and molecular mechanisms, such as aggregation and accumulation of disease-related proteins in specific regions of the brain. These include alpha-synuclein aggregates in Parkinson's disease and other synucleinopathies, inclusions of hyperphosphorylated microtubule-binding Tau in tauopathies, and extended polyglutamine protein aggregates (huntingtin) in Huntington's disease.

The association between the progression of clinical symptoms and the topographical distribution of pathology in neurodegenerative diseases has led to the hypothesis that specific pathological disease-related proteins can be transferred intercellularly in a prion-like manner. This hypothesis was created after the observation Lewy body pathology, a characteristic hallmark in synucleinopathies, within fetal dopaminergic neurons grafts in Parkinson's disease patients. Later, injection of Tau aggregates into animal models was shown to induce pathology. More recently, similar mechanisms were proposed to occur in monogenic forms of neurodegenerative diseases after the observation of mutant huntingtin aggregates within fetal striatal allografts in the brain of Huntington's disease patients. Furthermore, the presence of co-pathology in the brain of patients with distinct neurodegenerative diseases has implied that several proteins may overlap to contribute to the neuropathophysiology and can share common molecular mechanisms in neurodegeneration.

Several mechanisms have been suggested to contribute to the spreading of alphasynuclein, Tau and huntingtin pathology. These include diffusion through the plasma membrane, release via extracellular vesicles (as ectosomes and exosomes), misfoldedassociated protein secretion pathway, membrane carriers, membrane pores, tunnelling nanotubes, endocytosis, and receptor-mediated endocytosis. To date, it is unclear if alphasynuclein, Tau and huntingtin release to the extracellular milieu occurs through similar cellular mechanisms and their effect in receptor cells. Also, the role of protein secretion and their involvement in neuronal dysfunction and disease progression remains elusive. Further elucidation of these questions will permit a better understanding of protein propagation on disease pathogenesis in neurodegenerative diseases.

In recent years, extracellular vesicles have emerged as central mediators in intercellular communication under physiological and pathological conditions. Their heterogeneity and presence in several human biofluids have led to extensive research 
regarding their content and functional properties as relevant biomarkers in neurodegenerative diseases. In particular, ectosomes and exosomes are considered important carriers of misfolded proteins between cells in disease. These can be internalized in diverse cell types, although their effect in neuronal activity is unclear. Discernment between ectosomes and exosomes has been difficult due to the moderate differences in their physical properties and absence of reliable markers. While exosomes have been extensively studied in the field, the role of ectosomes in the pathogenesis of neurodegenerative diseases has not been explored. Additional studies focusing in the role of ectosomes and other types of extracellular vesicles in neurodegenerative diseases will open new avenues to uncover potential disease biomarkers and therapeutic targets.

In our first study, we developed a detailed differential ultracentrifugation protocol to efficiently purify ectosomes and exosomes, and provided a proteomic and functional characterization of these vesicles subtypes. Comprehensive proteomic analysis revealed specific protein composition and pathways enrichment for ectosomes and exosomes. Interestingly, ectosomes isolated from human cerebrospinal fluid and from cell media displayed enrichment in annexin-A2, suggesting this protein as an important marker for ectosomes characterization. Furthermore, treatment of neuronal cortical cultures with ectosomes and exosomes resulted in their internalization at similar ratios. Using multielectrode array technology, we further demonstrated that extracellular vesicles internalization affects differently the spontaneous activity of neuronal cells.

In our second study, we demonstrated that common cellular mechanisms are used for the transfer of alpha-synuclein, Tau and huntingtin exon-1 fragments between cells. Interestingly, we observed that these proteins are handled in different ways depending on the receptor cells. Our results reveal the release of the different disease-related proteins to the cell media at different levels in a free form and in extracellular vesicles. Overall, alphasynuclein, Tau and normal huntingtin exon-1 were found in higher levels in the cell media in contrast to mutant huntingtin exon-1. We further observed discernible alterations in the spontaneous firing activity in primary cortical neurons after treatment with the different recombinant proteins, suggesting that the effects of alpha-synuclein, Tau and huntingtin in the extracellular space and on neuronal activity are dependent of the protein properties and not only correlated with their secretion levels. Interestingly, alpha-synuclein, Tau and huntingtin exon-1 were present in higher levels in ectosomes than in exosomes. We revealed that these vesicles could be internalized in microglial and astrocytic cells, and resulted in the production of pro-inflammatory cytokines and autophagy activation. Neuronal cells also internalized ectosomes and exosomes enriched with alpha-synuclein, Tau or huntingtin, and exhibited irregularity in the cell bursting properties that overall was correlated with the vesicles subtype. 
Overall, our work indicates that extracellular vesicles cargoes likely reflect core pathophysiological intracellular processes in their origin cells. A clear understanding of the specific functional properties of different vesicles subtypes might represent a step forward in the search of novel biomarkers. Furthermore, our results propose that common cellular mechanisms are used for the transfer of alpha-synuclein, Tau and huntingtin between cells. These similarities could suggest common therapeutic targets for neurodegenerative diseases, and the need to target several cellular mechanisms to halt the detrimental effects of protein transmission and pathology progression. 


\section{Introduction}

The longer-life expectancy is increasing the prevalence of neurodegenerative diseases in the world population [1]. At present, several therapeutic interventions are available to prevent the onset of the symptoms and slow down the disease progression. However, the absence of a deep understanding of the causes and mechanisms of each disease and lack of diagnostic biomarkers hinders the early detection of these disorders [2]. Hence, the search of specific biomarkers in human biofluids and individual targets are a priority for the development of novel therapies.

Neurodegenerative disorders are generally classified by their clinical presentations, as cognitive and movement symptoms, with most patients having mixed clinical features [3]. Furthermore, these disorders share several central pathways associated with gradual cell dysfunction and death, such as oxidative stress, proteasomal and autophagosomal/ lysosomal degradation systems, and neuroinflammation [4]. Nonetheless, the protein abnormalities that characterize neurodegenerative diseases are typically developed before the onset of clinical features and more than one neurodegenerative process can be found in an individual $[5,6]$.

The most common neurodegenerative diseases include amyloidosis, tauopathies, synucleinopathies, and polyglutamine (polyQ) proteinopathies [7]. Individual neuropathologic diagnosis is performed through the observation of specific abnormal protein conformations and their cellular and anatomical distribution in the brain of patients with these disorders [4]. Assessment of post-mortem human brain tissue from patients with a variety of clinical and pathological features founded the elaboration of staging systems that describe the stereotypic progression of many neurodegenerative disorders. These include Alzheimer's disease (AD) [8, 9], Parkinson's disease (PD) [10, 11], and Huntington's disease (HD) staging schemes [12].

Most proteins have an inherent tendency to convert from their native functional state into a pathological intractable amyloid aggregate [7]. These include proteins known to have a physiological role in the cells and to be deposited as amyloid or other aggregate types in human disorders. Protein accumulations that constitute the major histopathologic features in neurodegenerative disorders include hyperphosphorylated tau in neurofibrillary tangles (NFTs), alpha-synuclein (aSyn) in Lewy bodies (LBs) and Lewy neurites (LNs), and mutant huntingtin ( $\mathrm{mHtt}$ ) in neuronal intranuclear and cytoplasmic inclusions. 


\subsection{Alpha-synuclein in Parkinson's disease and other synucleinopathies}

Neurodegenerative diseases are commonly associated with accumulation of pathological proteins, as aSyn in synucleinopathies [13, 14]. These diseases include PD, dementia with Lewy bodies, multiple system atrophy, neurodegeneration with brain iron accumulation type I, diffuse Lewy body disease and Lewy body variant of AD [15-18].

James Parkinson presented the first clinical description of PD over 200 years ago [19], with patients exhibiting motor symptoms as resting tremor, slow movements, rigidity and postural instability $[20,21]$. These symptoms result from a deregulation in the activity of the basal ganglia activity, due to the loss of dopaminergic neurons in the substantia nigra pars compacta [22]. Furthermore, patients also display numerous non-motor symptoms as cognitive impairment, hyposmia, rapid eye movement sleep disorder and constipation [23, 24]. Friedrich Lewy in 1912 described intracellular inclusion bodies as neuropathological hallmarks in PD [25]. Currently, it is known that LBs and LNs are enriched with aSyn and are involved in the pathogenesis of synucleinopathies $[15,26]$. aSyn has been the focus of intense investigation due to its central role in synucleinopathies since it is the main component of Lewy pathology, but it is also implicated in several cellular and molecular processes that contribute to PD pathology progression [14].

The synuclein family is composed with 3 members, alpha-, beta- and gamma-synuclein (a-, b- and gSyn, respectively) [27]. These small soluble proteins bind to phospholipid membranes and share a highly conserved alpha-helical lipid-binding domain [28]. Despite the similarity in their sequence, these proteins have different roles in the cells and display different biochemical properties. Synucleins are ubiquitously expressed in the human body and are particularly enriched in neurons. In particular, aSyn is predominantly expressed in the hippocampus, striatum, neocortex, cerebellum and thalamus [29, 30]. Intriguingly, knockout of the synuclein family in animal models did not result in major brain morphological changes [3135]. Nonetheless, the triple knockout model exhibited neuronal dysfunction, alterations in synaptic structure and transmission, and reduced survival, implying that synucleins contribute to the long-term synaptic function.

aSyn was firstly identified in the electric organ of Torpedo californica, being distributed in the nuclear envelope and in the presynaptic terminal [36]. In the brain, this protein is initially localized in the soma of immature neurons and afterwards is concentrated in the synapses at the presynaptic terminals in mature neurons [27, 37-40]. During aging, aSyn changes its localization to the soma, and is often interpreted as a pathology development marker in PD 
[41, 42]. Indeed, aSyn has been associated with numerous cellular and molecular processes that contribute to synucleinopathies, but whether it is a culprit or a bystander is still unknown [14]. Furthermore, several studies propose that aSyn has a physiological role in the maintenance of the synaptic function, particularly in the recycling of synaptic vesicles and in SNARE-complex assembly [35, 43, 44].

aSyn is a $14.5 \mathrm{kDa}$ acidic protein comprising 140 amino acid residues (Figure 1) [45]. Structurally, it is divided in three main domains: residues 1- 60 comprise the lipid binding alpha-helix $\mathrm{N}$-terminal, residues 61- 95 delimit the non-amyloid beta-component (NAC) domain, and residues 96- 140 define the unstructured C-terminal. Rare familial forms of PD are associated with missense mutations in the SNCA gene encoding for aSyn (A30P, A30G, E46K, H50Q, G51D, A53E, A53T) [46-52], as well as gene duplications and triplications (Figure 1) [53-55]. Additionally, polymorphisms in regulatory elements of the SNCA gene increase the predisposition for development of PD and have earlier disease onset [56]. aSyn missense mutations are located within the membrane-binding domain in the N-terminal and the lipid binding is only affected by A30P, G51D and A53E mutations [57-59]. The NAC region is relatively hydrophobic and prone to aggregation [60]. The acidic C-terminal is associated in multiple protein interactions, as with as ions and metals [61-64]. This terminal is unstructured and important for protection of aSyn aggregation $[65,66]$.

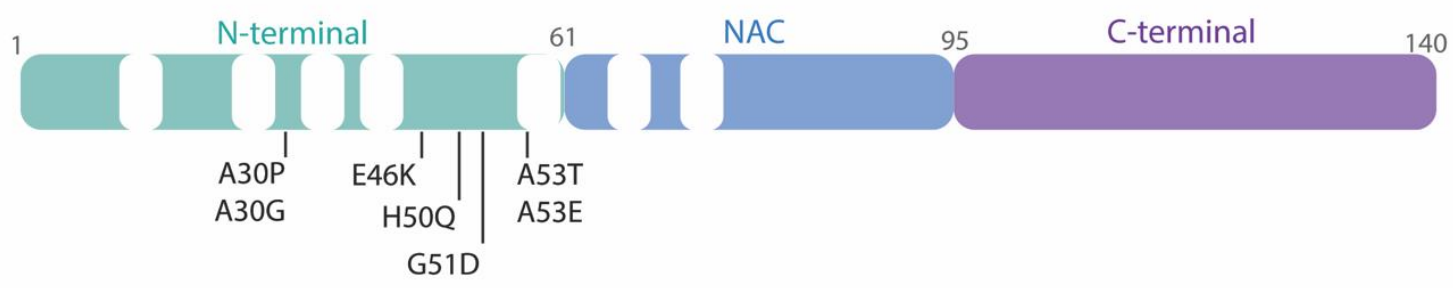

Figure 1. Schematic representation of aSyn structural domains and familial mutations. The N-terminal domain (amino acids 1- 60, in turquoise) displays the KTKEGV motifs (white rectangles) and contains the mutations sites associated with familial PD (A30P, A30G, E46K, H50Q, G51D, A53E, and A53T). Residues 61- 95 (blue) represent the non-amyloid- $\beta$ component (NAC) that is mainly hydrophobic and is involved in aSyn oligomerization process. The C-terminal (amino acids 96-140, purple) is highly acidic and is involved in the interaction with other proteins and metals. Also, truncations in this domain are linked with enhanced aggregation. Adapted from Brás IC, Outeiro TF. (2021) Alpha-synuclein spreading mechanisms in Parkinson's Disease: the role of membrane receptors. Elsevier. In revision.

Numerous post-translational modifications (PTMs) have been described in aSyn within the C-terminal. This includes truncation, phosphorylation, glycation, acetylation, glycosylation, oxidation, ubiquitination and nitration [67]. These modifications alter the protein charge and 
structure, resulting in alterations in the binding affinities with other proteins and lipids. Particularly, membrane binding, oligomerization and neurotoxicity are regulated through aSyn phosphorylation. The major phosphorylation sites are serine 87 and 129 (S87 and S129) [6873]. Furthermore, LBs are enriched in hyperphosphorylated aSyn at S129 and this PTM has been considered a pathological hallmark in PD [74-76].

Generally, aSyn exists in an equilibrium between a soluble and a membrane-bound state. Cytosolic aSyn is natively unfolded and soluble in cells [77-79], while when bound to membranes adopts an alpha-helical structure [80-83]. aSyn was also described to exist as a soluble tetrameric form, though this finding was not confirmed in other studies [84-86]. Additionally, aSyn oligomerizes into multimers upon membrane binding [87, 88]. Under pathological conditions, aSyn adopts a beta-sheet amyloid conformation commonly associated with its aggregation and neurotoxicity in cells (Figure 2) [89-93].

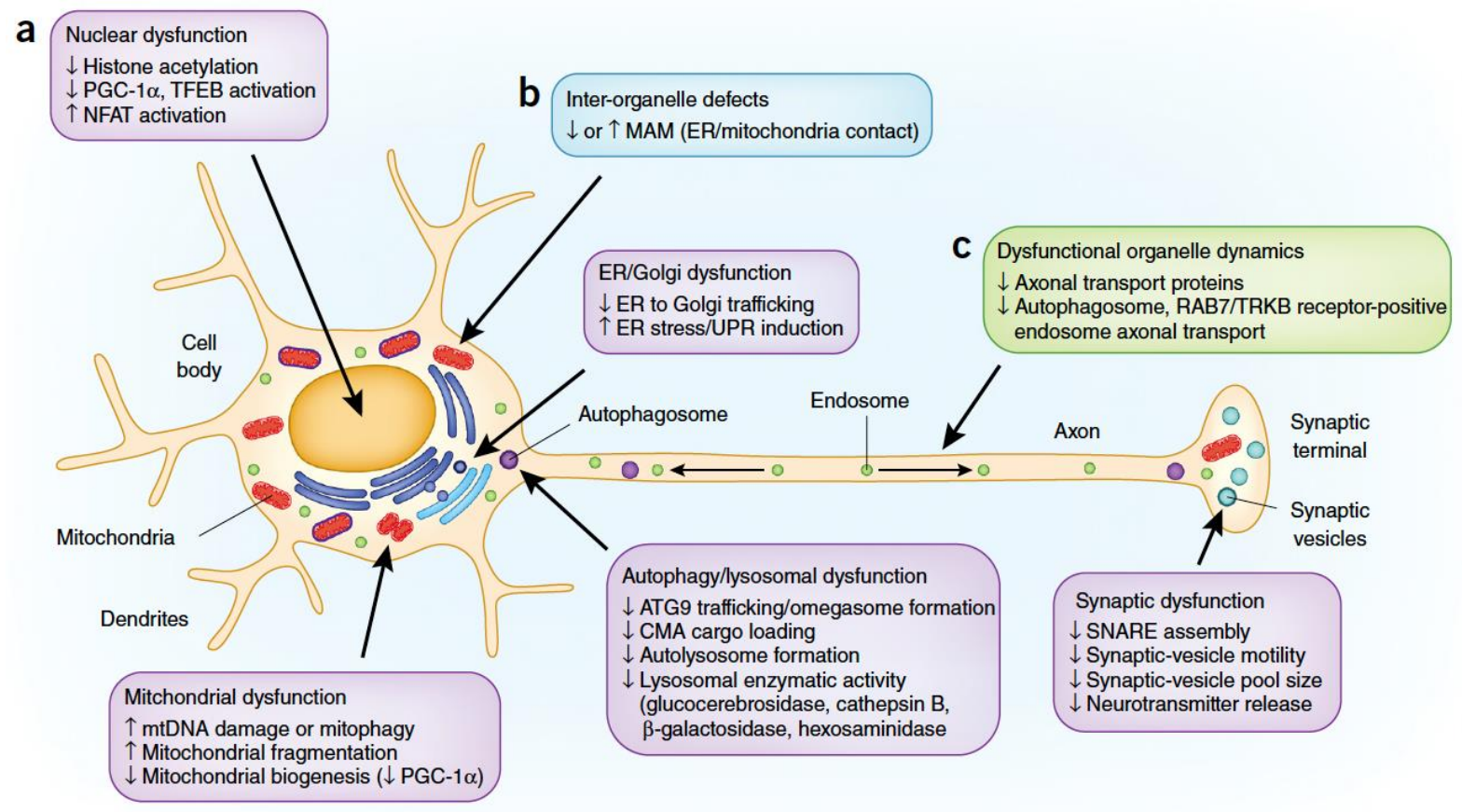

Figure 2. Several pathways are affected by aSyn misfolding and aggregation in synucleinopathies. aSyn toxicity in cells results in (A) organelle dysfunction (purple boxes), (B) alterations in inter-organelle connections (blue box) and (C) dysfunction in organelle dynamics (green box). Adapted from [94]. 


\subsection{Tau as a key player in tauopathies}

In 1907, Alois Alzheimer and Oskar Fischer discovered the characteristic amyloid plaques and neurofibrillary lesions in AD [95-99]. The structure and composition of these lesions remained unidentified until nearly 80 years later, when it was revealed that these NFTs were composed of Tau filaments with atypical phosphorylation [100-102]. Bernardino Ghetti and Michel Goedert introduced later the term tauopathies to categorize neurological disorders with deposition of Tau fibrils as their main neuropathological hallmark [103]. This group of heterogenous diseases includes Pick's disease, corticobasal degeneration, progressive supranuclear palsy, globular glial tauopathy and argyrophilic grain disease as primary tauopathies. $A D$ is considered a secondary tauopathy due to the deposition of Tau fibrils in combination with extracellular beta-amyloid plaques [104]. Primary tauopathies are a subgroup of the frontotemporal lobar degeneration (FTLD) disorders, a group of diseases that exhibit a predominant atrophy of the frontal and temporal lobes with accumulation of Tau inclusions [105]. Abundant filamentous Tau deposits often occur in neurons alongside with glial cells and are associated with severe neuronal toxicity and cell loss [106, 107]. Individuals with these disorders display diverse clinical symptoms and distinctive pathology distribution [104].

Tau was firstly perceived as a dull initiator of the toxic effects in AD pathology. Nowadays, this protein is considered a main factor in the development of several neurodegenerative diseases [104]. In AD, Braak proposed a disease staging system through the correlation of accumulation of Tau lesions with disease progression [8]. Development of Tau pathology follows in a hierarchical pattern of accumulation beginning from the layer II of entorhinal cortex and disseminating towards the cortical regions [8, 108]. Furthermore, advanced Tau pathology is often associated with increased cognitive impairment in tauopathies, and increased Tau levels in the cerebrospinal fluid (CSF) are associated with faster cognitive decline and worse clinical outcome in AD [9, 109-112].

Tau is a microtubule-associated protein (MAP) present in six major isoforms in the human brain which span from 352 to 441 amino acids (Figure 3) [113]. This protein is encoded by the MAPT gene and can undergo alternative splicing of exon 2 and 3 producing proteins with three amino terminal variants $(\mathrm{N})$, whereas alternative splicing of exon 10 generates isoforms with either 3 or 4 microtubule binding domains $(R)$ in the carboxyl terminal (ON3R, 1N3R, 2N3R, 0N4R, 1N4R, 2N4R) [104]. Only 0N3R Tau is expressed in the fetal human brain, in contrast with the adult brain where all the six isoforms are present with a $1: 1$ ratio of the $3 \mathrm{R}$ and $4 \mathrm{R}$ isoforms. Alterations in the proportion of the Tau isoforms can lead to its dysfunction and pathology development in cells $[114,115]$. In tauopathies, 3R Tau isoforms 
accumulate in Pick's disease while 4R Tau isoforms accumulate mainly in progressive supranuclear palsy, corticobasal degeneration, globular glial tauopathy and argyrophilic grain disease. Furthermore, both Tau isoforms accumulate in $\mathrm{AD}[116,117]$. Tau isoforms containing exon 2 and exon 10 are more prone to aggregation due to the existence of the VQIINK hexapeptide in exon 10 that mediates oligomerization $[118,119]$.

(a)
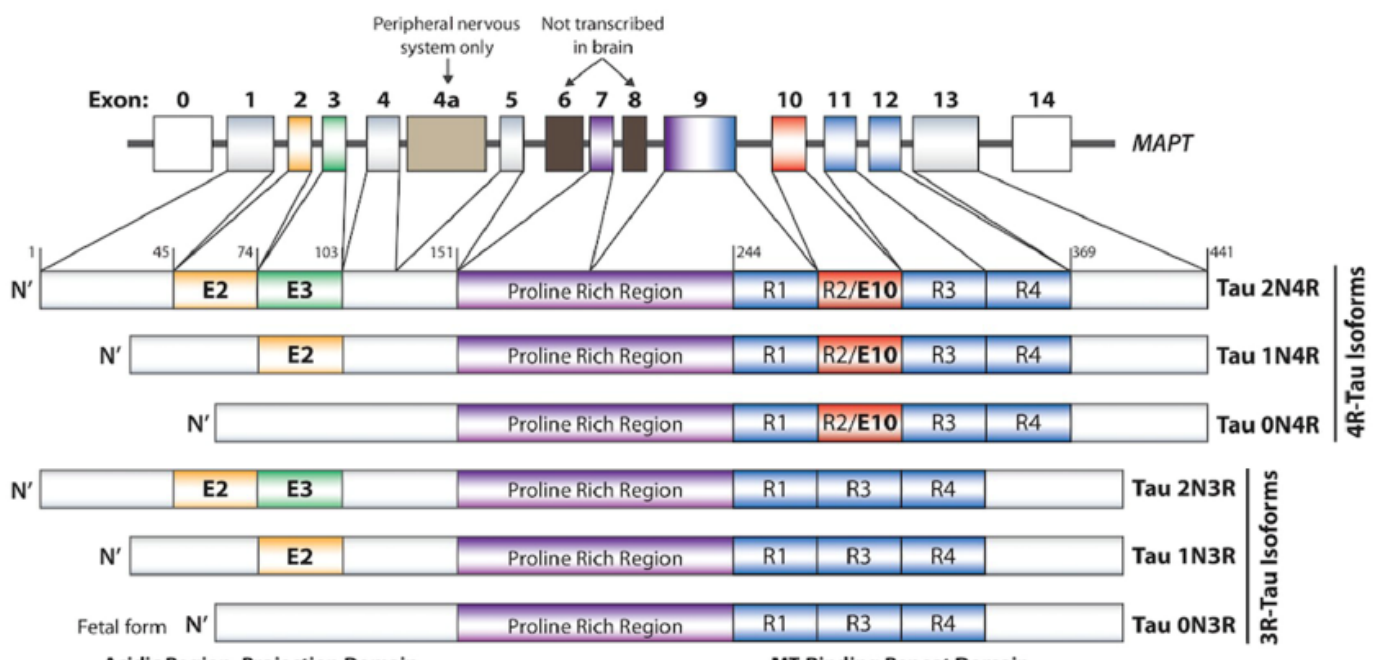

Acidic Region, Projection Domain MT-Binding Repeat Domain

(b)

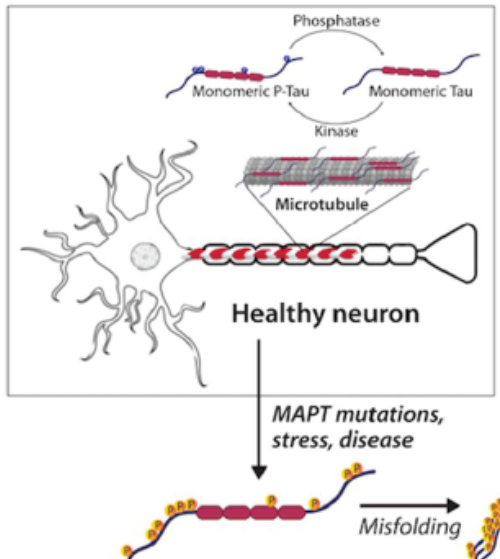

Hyperphosphorylated Tau
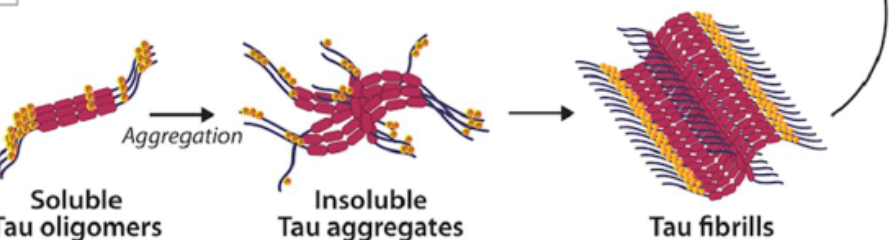

(c)

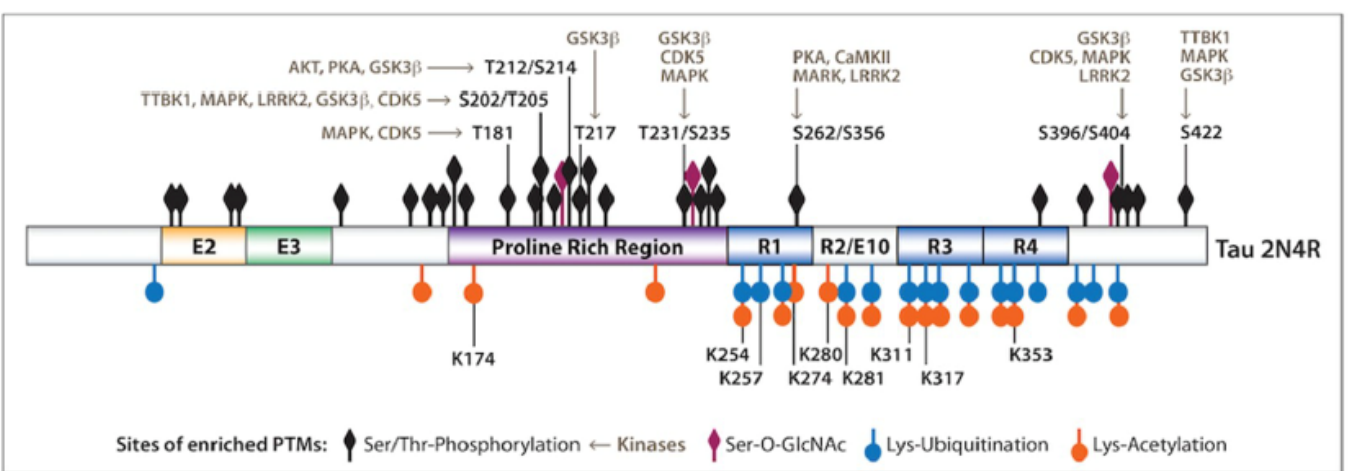

Figure 3. Physiological and pathological functions of human Tau. (A) MAPT gene undergoes alternative splicing and generates six Tau isoforms, comprising three (3R) or four (4R) microtubule (MT)-binding domains in 
the C-terminal, and zero- two N-terminal domains. (B) Tau regulates MT stability and dynamics in neuronal cells. Tau binding to the MT is regulated through its phosphorylation levels that are regulated by kinases and phosphatases. In pathological conditions, increase in Tau phosphorylation levels leads to its detachment from the MT, resulting in axonal dysfunction. The increase in other post-translational modifications (PTMs) drives Tau misfolding and aggregation into large insoluble fibrils termed neurofibrillary tangles (NFTs). (C) Tau contains numerous PTMs under physiological and pathological conditions, as phosphorylation, O-GlcNAcylation, acetylation, and ubiquitination. In particular, phosphorylation is intensified in tauopathies and is regulated by key regulatory kinases. Adapted from [120].

Structurally, Tau comprises four functional domains: the N-terminal projection domain, a proline-rich region, the microtubule binding domain, and the $\mathrm{C}$-terminal region (Figure 3 ). The N-terminal regulates microtubule binding despite not being directly involved in the physical interaction (residues 1-150 of the longest isoform), and when absent promotes Tau relocalization from the cytosol to the nucleus [121, 122]. This domain also mediates Tau localization in the plasma membrane through annexin-A2 interaction [123]. The proline-rich domain is the disordered central domain of the protein (residues 151-243) and this region interacts with Src homology-3 (SH3) proteins and nucleic acids [124-126]. The microtubulebinding repeat domains regulate Tau binding affinity to microtubules and actin for cytoskeleton stability (residues $244-441$ of the longest isoform). Several disease-associated proteins, as aSyn, FUS, TIA-1 and presenilin-1 interact with the microtubule-binding repeat and the prolinerich domains, suggesting that protein interactions with Tau can have a relevant role in pathology development [127]. In last, the C-terminal region is involved in the inhibition of Tau polymerization [128, 129].

In neurons, Tau is distributed throughout the neuron and it becomes enriched in the axon during maturation [130]. It can also be found in the plasma membrane, dendrites, synapses, nucleus, and mitochondria [131-135]. Tau has an important role in cytoskeletal organization and trafficking during microtubules formation, which is critical for the transport of cellular cargo between axonal and dendritic peripheries [136, 137]. Dysregulation of Tau interaction with microtubules leads to their disassembly and subsequent impairment in cell polarity and viability [138-140]. Several Tau domains interact with lipids and membranes, and this protein can also act as a signalling regulator via its interaction with membrane receptors [141-144]. In the synapse, Tau has been reported to be involved in synaptic development and neuronal activity [134, 145]. During pathology, Tau induces synaptic dysfunction and alterations in neuronal excitability [146-150]. Indeed, higher levels of phosphorylated Tau induced a higher depolarized threshold for action potential and reduced firing in neuronal cells [151, 152]. 
In 1997, the first familial mutations in the MAPT gene were associated to frontotemporal dementia and parkinsonism linked to chromosome 17 (FTDP-17), indicating that Tau mutations can lead to its aggregation and neurodegeneration in cells (Figure 3) [153155]. Nowadays, more than 50 pathogenic mutations in the MAPT gene were described to exist mostly located in exons 9- 12 [156]. These can modulate Tau microtubule binding properties (as K257T, G272V, P301L, V337M and R406W mutations), and change alternative splicing towards overproduction of the $4 \mathrm{R}$ isoform (as N279K, N296H, P301S and S305I mutations) [157, 158].

In physiological conditions, Tau is a natively unfolded protein and can adopt various structural states relevant to its function in cells [159, 160]. During pathological conditions, the protein conformation changes to beta-sheet and leads to the formation of filamentous structures [161, 162].

Tau undergoes a variety of PTMs, including phosphorylation, ubiquitination, oxidation, glycation, truncation, and glycosylation under physiological and pathological conditions (Figure 3) [163]. Enhancement of these modifications in tauopathies leads to Tau accumulation and aggregation [164]. Abnormal phosphorylation is considered the first step for Tau oligomerization and formation of NFTs [100, 165-167]. 


\subsection{Polyglutamine repeats in Huntington's disease and other monogenic neurodegenerative disorders}

Polyglutamine (polyQ) diseases are a diverse group of inherited neurodegenerative disorders caused by expansions of CAG repeats encoding polyQ tracts in specific genes [168, 169]. These include HD, spinocerebellar ataxia 1, 2, 3, 6 and 7, dentatorubral-pallidoluysian atrophy and spinal-bulbal muscular atrophy [170-179]. Disease onset is determined by the pathogenic polyQ length threshold and lead to slowly progressive phenotypes. In each case, the expanded repeat is unstable and can increase in length as it is passed from one generation to the next, resulting in earlier manifestation of the disease at younger ages in the subsequent generations. These disorders also share various clinical and pathological features despite having only in common the genetic pathogenic repeat expansion in differently affected genes $[180,181]$. A common pathological hallmark in polyQ diseases is the accumulation of the polyQ proteins as intracellular aggregates predominantly in the nucleus [168, 182, 183].

In 1872, George Huntington described an progressive hereditary chorea with the phrase "Once it begins it clings to the bitter end" [184]. Nowadays, this disease is known as $H D$, a dominantly inherited genetic neurodegenerative disorder usually manifested during adulthood and that progressively worsens over a period of $10-30$ years $[185,186]$. The cardinal neuropathological hallmark of this disease is a massive atrophy of the caudate and putamen regions culminating in neuronal dysfunction and cell loss [187, 188]. Striatal medium spiny neurons are most vulnerable to the $\mathrm{mHtt}$ toxicity, though considerable neuronal dysfunction and cell death also occurs in the cortex [189, 190]. Patients often exhibit alterations in personality, cognitive deficits, and involuntary movements [191-194]. Also, several studies have described peripheral symptoms including muscle degeneration, weight loss, and activation of inflammatory processes [195]. Due to the absence of treatments that can prevent or slow down HD progression, the clinical care of patients focuses on management of the symptoms through medical treatments to extend function and quality of life [186].

The length of the CAG repeats in the exon 1 of the huntingtin gene $(H T T)$ on chromosome 4, which encodes for the protein huntingtin $(\mathrm{Htt})$, determines whether an individual will develop or not HD $[196,197]$. The CAG repeat is polymorphic in the normal population with the range between 6 - 35 repeats. Individuals with 36- 39 CAG repeats show reduced penetrance, as some individuals live a normal lifespan without being clinically diagnosed or they can manifest the disorder [198]. The disease pathology is exhibited when an individual possesses a CAG expansion higher than 40 , that leads to the production of the toxic and mutant form of the huntingtin protein with an expanded polyglutamine stretch at the 
$\mathrm{N}$-terminal, which is prone to misfold and aggregate in cells [193, 199]. Numerous proteases cleave $\mathrm{mHtt}$ and generate exon $1 \mathrm{~N}$-terminal polyglutamine fragments, which subsequently interact with other proteins and lead to the formation of toxic $\mathrm{mHtt}$ aggregates [186, 200-203].

Normal $\mathrm{Htt}$ protein has polyQ repeat length of 23 glutamines with a total of 3144 amino acids and a molecular weight of $348 \mathrm{kDa}$ (Figure 4) [204]. The full-length $\mathrm{Htt}$ exhibits a compact shape with three domains: N-terminal, a central domain and C-terminal [204]. The N-terminal domain contains the expandable polyQ stretch, preceded of 17 amino acids, and followed by a proline-rich domain (PRD). The 17 amino acids region consists of an amphipathic alphahelix, important for Htt retention in the ER [205, 206]. Furthermore, it functions as a nuclear export signal (NES) and is subject to several PTMs as acetylation, ubiquitination, and sumoylation that modulate $\mathrm{Htt}$ degradation and subcellular localization [205, 207-209]. The PRD domain seems to be important for mediating protein-protein interaction [210]. The central domain consists mainly of supercoiled alpha-helical structures termed HEAT repeats important for protein-protein interaction and mediating inter- and intra-molecular interactions [211]. The HEAT repeats are separated by regions with disordered structure known to undergo several PTMs, as proteolytic cleavage, phosphorylation, and glycosylation [212, 213]. Lastly, $\mathrm{Htt}$ also contains other functional motifs such as nuclear localization signal (NLS) and NES for protein shuttling between nucleus and cytoplasm via active transport [214-216].

Htt is involved in the development of the central nervous system (CNS), as neural tube formation and neuroblast migration, and animal models with $\mathrm{Htt}$ deletion die before birth [217, 218]. This protein is involved in several cellular and biological functions such as transcription, cell division, cell survival, axonal transport, vesicular trafficking, synaptic function, and energy metabolism [204, 219]. Htt is expressed throughout the body, with higher levels in the brain. In cells, it localizes in the nucleus, cytoplasm, mitochondria, endoplasmic reticulum (ER), Golgi complex, endosomes, and synaptic vesicles [219, 220]. The polyQ tract length affects the PTMs distribution, which influences the stability, cleavage, subcellular distribution, and function of $\mathrm{Htt}$ (Figure 4) [221]. This protein also binds and interacts with DNA, and the expanded polyQ tract results in transcriptional dysregulation [222]. This leads to upregulation of the immune response and mRNA processing, and downregulation of metabolic processes and synaptic function [223]. 


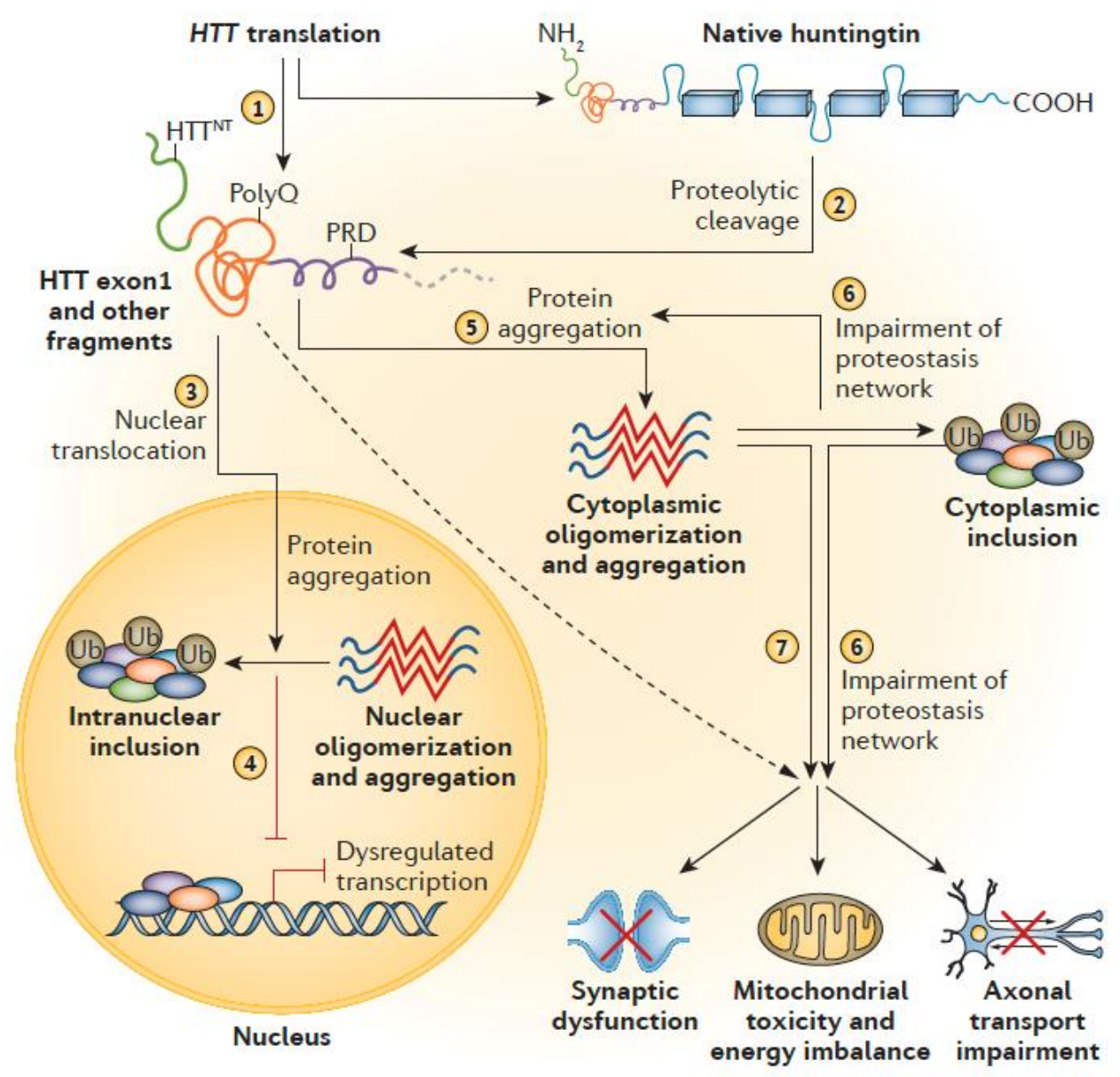

Figure 4. Cellular mechanisms involved in the pathogenicity of mutant huntingtin (HTT) in Huntington's disease (HD). (1) Translation of HTT gene generates the full-length or the N-terminal exon1 fragment HTT protein (as a result of alternative splicing). (2) Proteolytic cleavage of full-length huntingtin generates protein fragments that can (3) enter the nucleus. (4) Formation of HTT inclusions in the nucleus, through protein oligomerisation and aggregation, causes transcriptional dysregulation. (5) HTT aggregates in the cytoplasm that exacerbated due to (6) impairment in the proteostasis network, which leads to (7) impairment in synaptic dysfunction, mitochondrial toxicity and axonal transport. PRD- proline-rich domain; Ub- ubiquitin. Adapted from [186].

In HD, the expanded polyQ tract causes abnormal folding of Htt, which leads to the formation of oligomers that act as seeds to the formation of fibrils and inclusions in cells (Figure 4) [224, 225]. The fibrillar aggregates have amyloid-like properties and a central beta-strand rich core [226-228]. More recently, studies demonstrated that $\mathrm{N}$-terminal $\mathrm{mHtt}$ oligomers are toxic and that the consequent inclusions formation can be protective in cells [229-231]. Also, large $\mathrm{mHtt}$ inclusions can occur without cell death [229, 232, 233]. Aggregation of $\mathrm{N}$-terminal $\mathrm{mHtt}$ fragments produced by proteolytic cleavage or abnormal splicing aggregate faster than the full-length protein in the brain of HD patients [234-236]. 


\subsection{Prion diseases, protein misfolding disorders and prionoids}

The concept of prion as disease triggering agent was created after the observation of neurodegeneration in animals exposed to "small proteinaceous infectious particle which is resistant to inactivation" [237]. Prions are infectious protein assemblies capable of transmitting and propagating a disease throughout the brain, and can be transmitted among individuals from the same or different species [237].

Transmissible spongiform encephalopathies (TSE) are a group of neurodegenerative diseases characterized by the misfolding and accumulation of the scrapie prion protein ( $\left.\mathrm{PrPs}^{\mathrm{Sc}}\right)$ [238]. PrPSc results from the aberrant folding of the endogenous prion protein $\left(\mathrm{PrP}^{\mathrm{C}}\right)$, or alternatively from mutations, repetitions, or truncations in the PRNP gene [239, 240]. This was the first protein described to have infectious properties and has exhibited the highest capacity to propagate neurodegeneration [241]. Creutzfeldt-Jakob disease is a well-known TSE as it can be initiated by consumption of beef from animal infected with bovine spongiform encephalopathy (also identified as "mad cow disease"). In addition, numerous genetic mutations in the PRNP gene are associated with distinctive TSE, as familial CreutzfeldtJakob's disease, fatal familial insomnia, prion systemic amyloidosis and GerstmannSträussler- Scheinker syndrome [240, 242]. PrPsc are highly stable proteins that accumulate in the CNS and lead to spongiform degeneration and neuronal loss [243]. Although the period of incubation can be from months to years, the clinical symptoms progress quickly and comprise motor dysfunction, ataxia, behavioural abnormalities and cognitive impairment [244].

Cellular $\mathrm{PrP}^{\mathrm{C}}$ is a glycosylphosphatidylinositol (GPI) anchored protein and contains two N-linked glycosylation sites [245]. Mature $\mathrm{PrP}^{\mathrm{C}}$ is present at the surface of the plasma membrane in lipid-enriched microdomains, and structurally can be divided in a disordered $\mathrm{N}$ terminal domain and a globular C-terminal domain composed of three alpha-helices and a short anti-parallel beta-pleated sheet [246, 247]. Several functions have been attributed to $\operatorname{PrP}^{\mathrm{C}}$ [248], as neural development, synapse formation, myelin maintenance, and signalling [249-252]. Also, post-natal deletion of $\operatorname{PrP}^{\mathrm{C}}$ does not lead to neuronal death [253].

Since PrPsc is the origin of distinct TSE, the clinical variability in these disorders has been considered to arise from unique misfolded protein conformations, termed as strains (Figure 5). These protein species display different degrees of infectivity and can induce a conformational change in the endogenous protein, perpetuating the pathology throughout the brain $[254,255]$. Furthermore, peripheral infection can contribute to the development of the disease in the CNS [256]. 


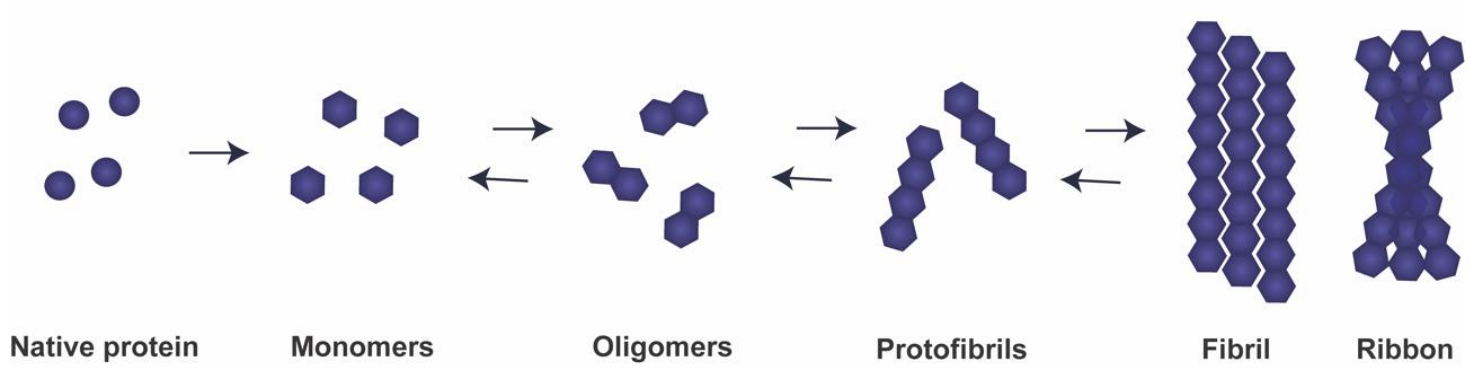

Figure 5. Proposed templated misfolding of disease-related proteins in neurodegenerative diseases. Due to stress, environmental factors, or genetic mutations, native protein monomers can self-aggregate into pathological oligomers. These species can be extended into protofibrils and other mature species as fibrils or ribbons that deposit into inclusions in the cells. These diverse assemblies coexist in a dynamic equilibrium and can be converted into higher- or lower-order conformations. Adapted from [257].

A key event in the pathophysiology of prion diseases is the template-directed misfolding of $\mathrm{PrP}^{\mathrm{C}}$ into a pathogenic beta-sheet-rich form ( $\mathrm{PrP}^{\mathrm{Sc}}$ ) [258]. The pathogenic protein conformation is a crucial element for the infectivity and transmission of prion diseases. However, the mechanism that underlies $\mathrm{PrP}^{\mathrm{C}}$ conversion into $\mathrm{PrP}^{\mathrm{Sc}}$ remains unknown [259]. It has been proposed that short segments of $\mathrm{PrPSc}^{\mathrm{Sc}}$ can interact with $\mathrm{PrP}^{\mathrm{C}}$ in a steric zipper, in which complementary amino acid side chains from two beta-sheets are tightly interdigitated and efficiently stabilize growing fibrils, mainly through hydrogen bonds [260, 261]. In fact, differences within the sequence of the steric zipper segments blocks the $\mathrm{PrP}^{\mathrm{C}}$ conversion between species $[262,263]$. Conversion mainly occurs on the plasma membrane or within the endocytic pathway in the multivesicular bodies (MVBs) [264]. In the brain, PrPsc spreads throughout anatomically connected brain regions [265]. However, the mechanisms involved in the transmission are poorly understood. In vitro studies propose that prions can be transferred from cell-to-cell via several cellular mechanisms, as extracellular vesicles (EVs) [266-270].

In 1987, Stanley Prusiner proposed that other neurodegenerative diseases could have prion-like properties [271, 272]. More recently, several studies demonstrated that protein strains are not unique to $\mathrm{PrP}^{\mathrm{Sc}}$, as similar findings have been observed for aSyn [273-276], Tau [277, 278], and Htt [279, 280] (Figure 5- 6). 


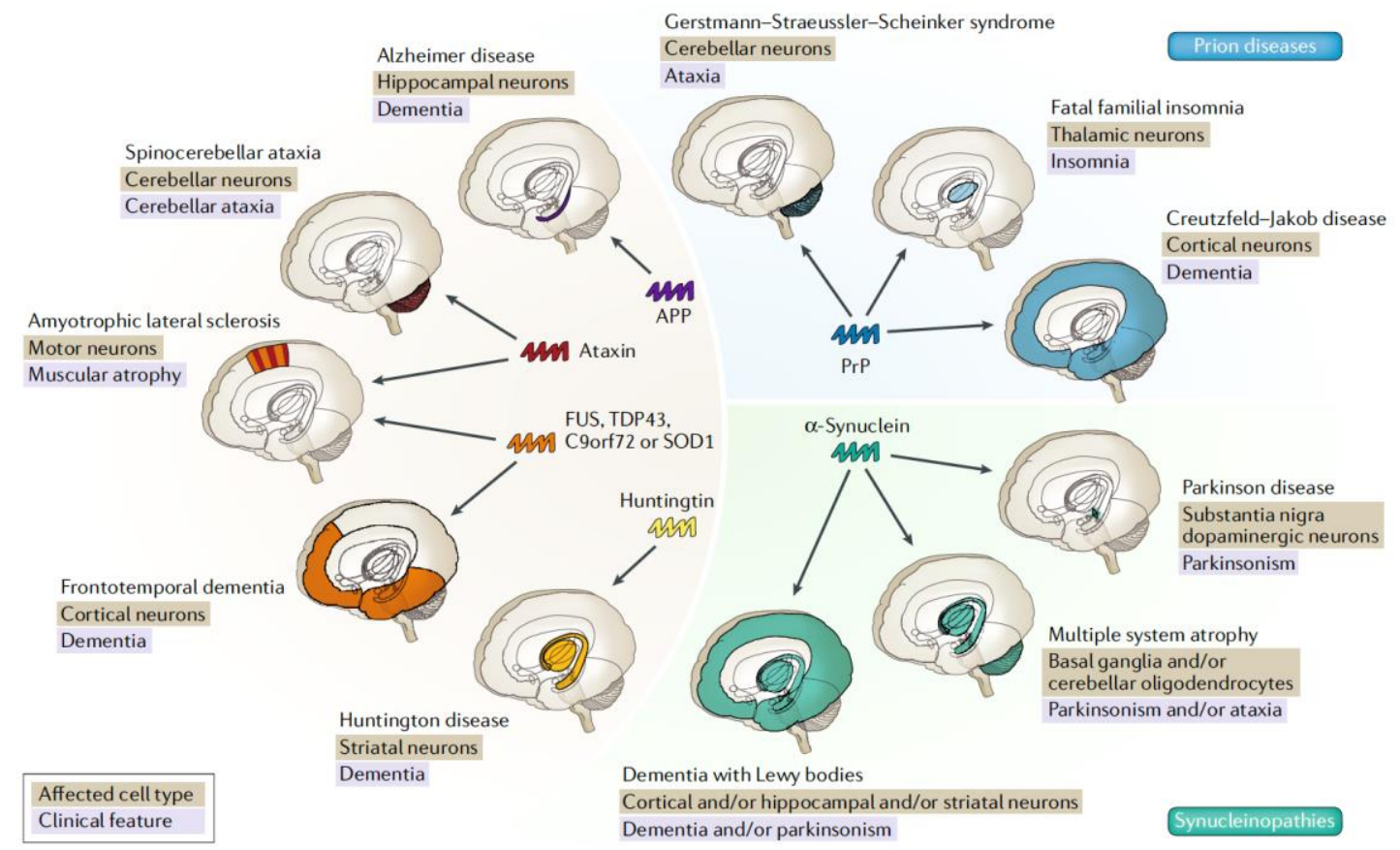

Figure 6. Protein misfolding disorders, prionoids and prions. Misfolding and accumulation of disease-related proteins is a common feature in several neurodegenerative diseases. This includes cellular prion protein (PrP), alpha-synuclein ( $\alpha$-Synuclein), amyloid precursor protein (APP), ataxin, RNA- binding protein (FUS), TAR DNAbinding protein 43 (TDP43), guanine nucleotide exchange (C9orf72), superoxide dismutase (SOD1), huntingtin and Tau. Aging, stress, and environmental conditions can trigger the misfolding of these proteins into toxic species. Remarkably, the same protein can be involved in different diseases exhibiting a spectrum of neuropathological and clinical symptoms, indicating the existence of diverse protein strains that exert toxicity in distinct manners. Adapted from [272].

Similarities between several neurodegenerative diseases with prion disorders led to their designation as prion-like and the respective disease-related proteins as prionoid proteins $[272,281]$. This includes the abnormal folding of endogenous protein into different strains via a template protein, transfer of misfolded proteins between cells and pathology propagation in the brain throughout interconnected regions (Figure 5- 6) [257, 282, 283]. However, the infectivity of these aggregates by successive transmission across hosts has not been proven yet. 


\subsection{The role of prion-like spreading mechanisms in the progression of neurodegenerative diseases}

Proteins involved in neurodegenerative diseases have been proposed to acquire their pathogenicity by a prion-like mechanism [284, 285]. Their self-propagation and transmission cell-to-cell seem to play an important role in disease progression, but it may also have a biological function that is not yet known [258, 286]. Histopathological analyses indicate that proteinaceous lesions develop in a disease-specific spatiotemporal pattern and through connected regions [265, 287].

Several mechanisms have been associated with transmission of aSyn, Tau and $\mathrm{Htt}$ between cells, as passive diffusion through the plasma membrane, misfolding-associated protein secretion pathway (MAPS), tunnelling nanotubes (TNTs), EVs as ectosomes and exosomes, membrane carrier proteins and release via pore-like structures [257, 282, 283]. Seeds can also be released by cells with damaged cell membrane (Figure 7).

The MAPS pathway was recently described to export aberrant cytosolic proteins using the ER-associated deubiquitylase USP19 [288]. Misfolded proteins are recruited to the ER surface for deubiquitylation, and later its encapsulated into late endosomes and secreted to the extracellular space (Figure 7). This protein quality control pathway promotes protein homeostasis by exporting misfolded proteins through an unconventional protein secretion process [288].

TNTs are F-actin membranous structures that allow the direct communication between the cytoplasm of distant cells (Figure 7) [289, 290]. These connections can be either connexinpositive gap junction-ended protrusions or open-ended [291], and allow the transfer of several cargoes, as proteins and cellular components [292]. However, it is unknown if these structures exist in the human brain or in vivo in animal models.

The misfolded proteins are then internalized by other cells via direct diffusion through the plasma membrane, endocytosis, or receptor- mediated endocytosis, or fusion of the EVs with the cell membrane (Figure 7). Pathological seeds can also interact with membrane receptors that can activate downstream signalling pathways or stimulate their internalization in the cells. Despite intensive research, the factors that modulate the molecular mechanisms involved in proteins transmission are not well understood. 


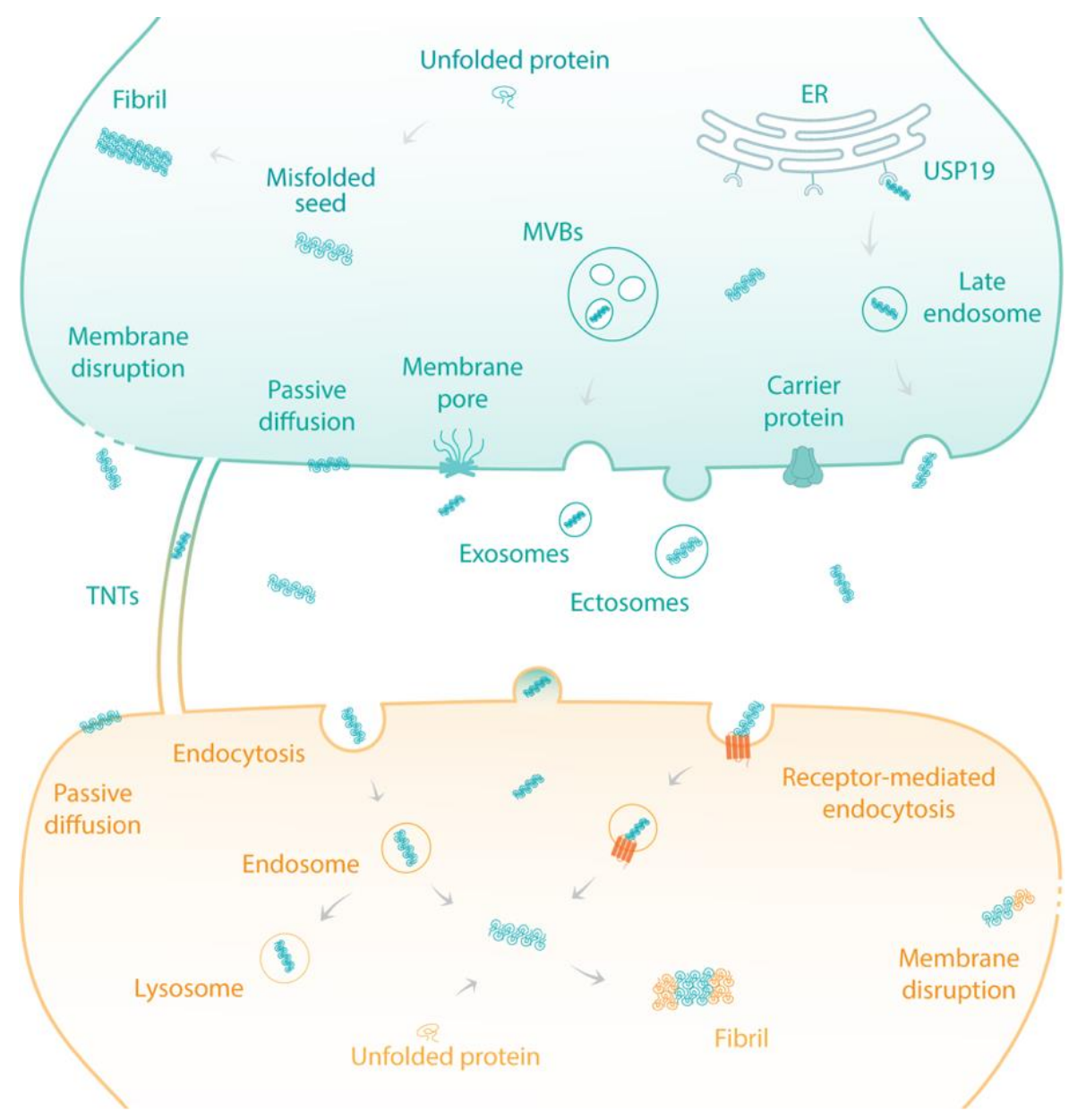

Figure 7. Proposed mechanisms involved with transmission of disease-related proteins between cells. Under pathological conditions, monomeric proteins can self-aggregate into pathological misfolded seeds and accumulate as mature fibrils in cells. Several mechanisms have been proposed to be involved in the release and internalization of aSyn, Tau and Htt assemblies. Misfolded proteins can be released via passive diffusion through the plasma membrane but can also be directed to the ER to be deubiquitylated by USP19 and encapsulated into late endosomes to be secreted to the cell exterior [misfolding-associated protein secretion pathway (MAPS)]. Misfolded seeds were described to be transferred using tunnelling nanotubes, secreted into extracellular vesicles (as ectosomes and exosomes), or be released by cells with damaged cell membrane. In particular, aSyn was described to be released via pore-like structures that penetrate in the cell membrane (there is no evidence that these structures exist in vivo) or use membrane carrier proteins. In the extracellular space, the released seeds can then be internalized by diverse cell types. This includes direct diffusion through the plasma membrane, endocytosis or receptor- mediated endocytosis, or fusion of the exosomal/ ectosomal vesicles with the cell membrane. Misfolded proteins can also interact with membrane receptors that might activate downstream signalling pathways or stimulate its internalization in the cells. After internalization via endocytosis, pathological seeds can disrupt the endosomal membrane to gain access to the cytosol or be carried for degradation. Free seeds can interact with endogenous proteins and template its fibrillization. This interaction can lead to potential disruption of the cell membrane and seeds release to the extracellular space, restarting the toxic vicious cycle. Adapted from Brás IC, Outeiro TF. (2021) Alpha-synuclein spreading mechanisms in Parkinson's Disease: the role of membrane receptors. Elsevier. In revision. 


\subsubsection{Extracellular vesicles as relevant mediators in intercellular communication in physiological and pathological conditions}

EVs mediate long range signalling events in the CNS and are essential vehicles in intercellular communication (Figure 7) [293-296]. These vesicles are secreted by different cell types and can be found in several biofluids, such as blood and CSF $[297,298]$. Nuclei acids, proteins, and lipids are incorporated into EVs and often used for the search of novel biomarkers in diseases (Figure 8) [299-301]. The three main EVs subtypes are apoptotic bodies, microvesicles (as ectosomes) and exosomes, which differ in their mechanisms of biogenesis, function, content and size [302, 303]. Their diversity suggests the possibility that cells secrete numerous different types of vesicles [304].

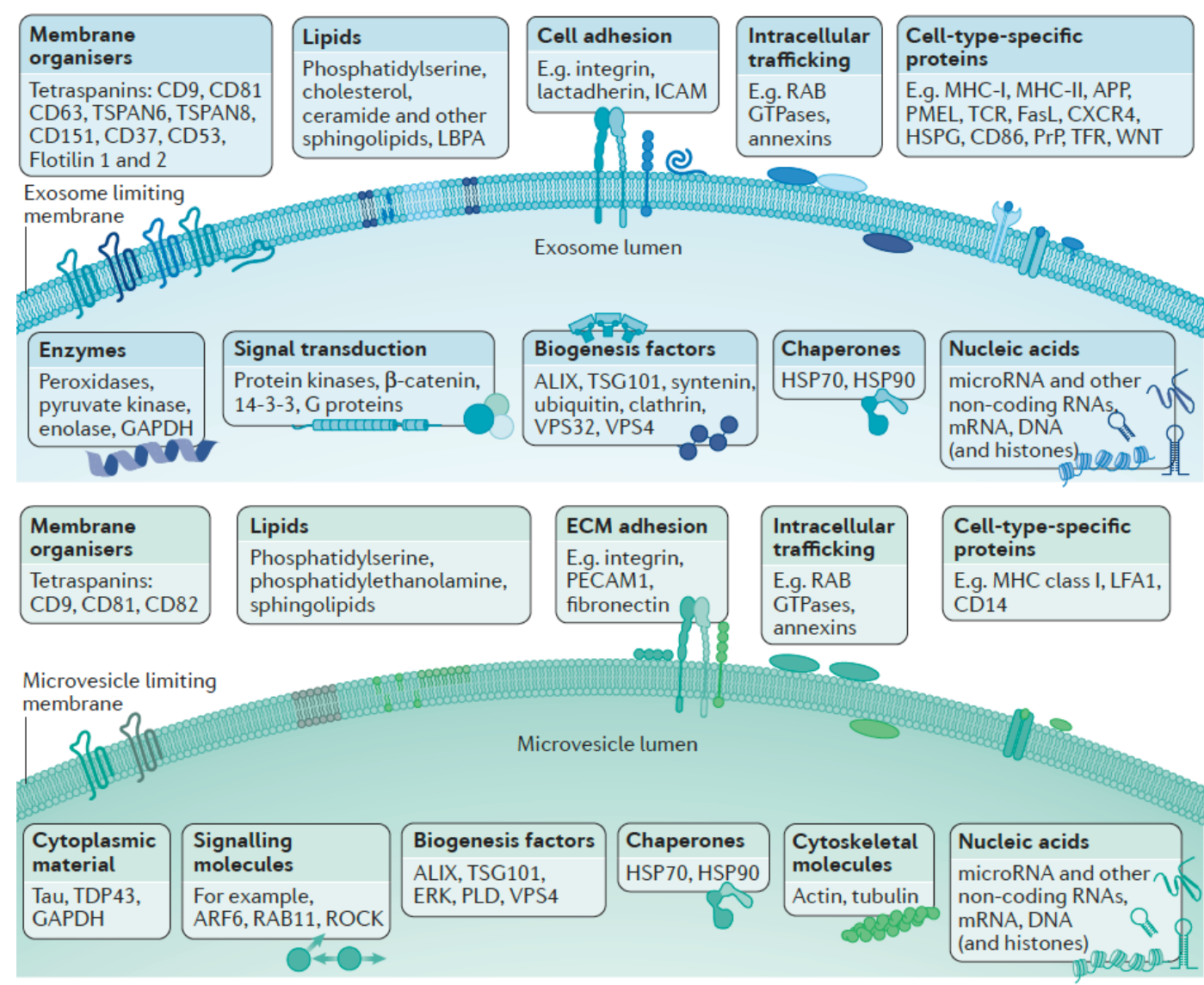

Figure 8. Exosomes and microvesicles transport numerous cargoes, as lipids, proteins and nucleic acids. The specific composition of extracellular vesicles (EVs) determines their function and fate, and changes in accordance with the cellular environment and cell type. This includes exosomes (top image, blue) and microvesicles (bottom image, green). The existence of varied EVs subtypes emphasizes the importance of selective cargo-sorting mechanisms that allow the transfer of different cargoes between cells. Despite distinctive mechanisms of biogenesis, exosomes and microvesicles contain several proteins in common, highlighting the importance of deeper characterization of EVs subtypes. APP- amyloid precursor protein; ARF6- ADP-ribosylation factor 6; ARMMs-arrestin-domain-containing protein 1-mediated microvesicles; CXCR4, CXC-chemokine receptor 
4; GAPDH, glyceraldehyde-3-phosphate dehydrogenase; HSP70- heat shock 70 kDa protein; HSPG- heparan sulfate proteoglycan; ICAM- intercellular adhesion molecule; LBPA- lyso-bis-phosphatidyl acid; LFA1- lymphocyte function-associated antigen 1; MHC- major histocompatibility complex; PECAM1- platelet endothelial cell adhesion molecule; PLD- phospholipase D; PrP- prion protein; ROCK- RHO-associated protein kinase; TCR- T cell receptor; TDP43- TAR DNA-binding protein 43; TFR- transferrin receptor; TSG101- tumour susceptibility gene 101 protein; TSPAN- tetraspanin; VPS- vacuolar protein sorting-associated protein. Adapted from [295].

Exosomes (30 to $150 \mathrm{~nm}$ in diameter) originate from endosomes released from MVBs after fusion with the plasma membrane [305, 306]. In contrast, microvesicles (also termed ectosomes), have a diameter between 100 to $300 \mathrm{~nm}$ and are generated by direct shedding from the plasma membrane [307, 308]. While exosomes have been extensively characterized in multiple studies, ectosomes proteomic and functional characterization remains understudied. Additionally, discrepancies in the purification strategies and lack of reliable protein markers that can discriminate between EVs types limits the understanding regarding their physiological and pathological functions [295, 299, 309].

The nature and abundance of EVs cargoes are determined by the cell type of origin and can be modified according to the physiological or pathological conditions in the cells [310]. Furthermore, several of the proteins that are considered as cargoes are involved in the regulation of EVs biogenesis (Figure 8) [295]. Once released into the extracellular space, EVs reach the recipient cells and deliver their content to promote physiological or pathological responses [295].

The presence of disease-related proteins in EVs highlight their potential roles in the spreading of the pathology in neurodegenerative disease. In contrast, these vesicles can act as vehicles for clearing of the toxic proteins associated with disease pathophysiology. They can further transport other pathogenic/ toxicity-inducing elements correlated with the disease. The functional duality between protective and pathological roles of EVs has not been fully addressed in the field [311].

Recently, EVs have been considered potential non-invasive biomarkers in several neurodegenerative diseases. Their presence in diverse human biofluids and stability highlights EVs as attractive targets for disease diagnostics [312, 313]. Furthermore, alterations in their content with aging might reflect the different stages of disease progression, providing the possibility of early diagnostic or monitoring the effect of current therapies [314-316]. To date, only a few studies addressed ectosomes and their composition in the search for biomarkers $[317,318]$.

Additionally, due to their ability to cross the blood-brain barrier, promising therapeutic drug carriers are being developed based on EVs into the CNS [319-321]. For example, the 
loading of nucleic acids, protein fragments or chemical compounds that can rescue the neurotoxic processes occurring in the disease context [322, 323].

Further research regarding the biogenesis, structure, cargo, and target cells of different EVs subtypes in physiological conditions and their alteration in neurodegenerative diseases will provide novel insights into their role in disease modulation and importantly clarify their potential as a source of disease biomarkers [324].

\subsubsection{Propagation of aSyn pathology}

The initial seeding capacity of misfolded aSyn was described in reports where PD patients who had received fetal nigral tissue grafts in the striatum exhibited Lewy pathology identical to the one observed in the patient tissue [325-333]. These results implied that grafted neurons inherited aSyn pathology from the host neurons.

Assessment of post-mortem brain samples of PD patients showed that Lewy pathology develops in a temporally and spatially expected manner. At early disease stages, aSyn pathology develops in the dorsal motor nucleus of the vagus nerve, which is connected to the enteric nervous system (ENS), and in the olfactory bulb. Afterwards, pathology progresses to the brainstem and cortical areas [10, 11, 334]. Further observations lead to the suggestion that PD could be initiated in the olfactory bulb or via the gastric route, and move upwards to the dorsal motor nucleus of the vagus nerve and finally travel through the CNS [335]. This proposal was also supported with the observation of Lewy pathology in the enteric and peripheral nervous systems [336, 337].

Experimental studies in vitro and in vivo subsequently provided further support for the self-propagation of aSyn seeds [273-276, 338-341]. Injection of brain-derived or recombinant aSyn seeds recapitulated some of the features observed in PD patients [341-344]. In addition, aSyn pathology was observed to propagate through the brain along anatomically connected regions, suggesting selective neuronal transport [341, 343, 345]. Peripheral injection of aSyn seeds can also induce pathology in the brain [346-348]. aSyn can also be found in the media of cell lines and primary neuronal cultures expressing this protein [349, 350].

aSyn was originally considered to be an exclusively intracellular protein due to the absence of a classical secretory signal peptide at the N-terminal. Later, aSyn was found in CSF and plasma in PD patients [351-353]. Therefore, it uses unconventional exocytosis pathways to be release to the extracellular space [354, 355]. Numerous studies are consistent with the use of prion-like spreading mechanisms for the transmission of aSyn pathology [257, 356]. These include passive diffusion [357-359], MAPS pathway [288], exosomes [349, 360- 
364], tunnelling nanotubes [365, 366], membrane pores [367], membrane carrier proteins [368], exo- and endocytosis [349] and receptor-mediated endocytosis [369]. The interaction of aSyn assemblies with lipid membranes can create annular pore-like structure in the plasma membrane [92, 367, 370, 371]. However, it is not known if these structures exist in vivo. Furthermore, membrane carrier proteins regulate membrane trafficking in cells and were described to participate in the release of aSyn [368].

Exosomes have been widely studied in PD. The transport of aSyn in these vesicles was shown to induce aSyn aggregation due to their lipid and protein composition [362, 364, 372, 373]. Exosomes internalization can induce protein aggregation along with a neuroinflammatory response [374]. Also, aSyn associated with exosomes appears to be preferentially internalized by cells when compared with free aSyn [375]. Injection of exosomes derived from various biofluids results in different functional effects in animal models, emphasizing that EVs content might change depending on their biofluid of origin [376, 377]. However, the correlation between the levels of aSyn in exosomes and disease severity has been inconsistent. While vesicles purified from CSF of early-stage PD patients exhibited reduced levels of aSyn compared to controls, exosomes purified from plasma displayed elevated levels of aSyn [373, 378].

Different aSyn strains can be spread between cells, conceiving a possible explanation for the heterogeneity observed in synucleinopathies [379]. However, there are several observations that contradict the prion-like hypothesis. For example, PD cases that do not present a typical pattern of Lewy pathology consistent with Braak staging [380-382]. Also, distribution of aSyn aggregates without correlation between the stage of Lewy pathology and clinical progression in patients [383-385]. Moreover, aSyn capacity to propagate from cell-tocell and between individuals is a matter of debate, since there is still no evidence of disease propagation between individuals, and several studies do not show pathology in grafted cells $[386,387]$. Other possible explanations for the manifestation of pathology in transplanted cells are stress conditions or alterations in the cellular milieu surrounding the graft that may trigger aSyn pathology [388].

\subsubsection{Propagation of Tau pathology}

Tau, the major component of NFTs in AD and other tauopathies, has also the capacity to self-assemble and propagate through prion-like molecular processes [277, 278, 389-399].

Intracerebral injection of Tau seeds into transgenic mice expressing human Tau or wild-type animals leads to the accumulation of hyperphosphorylated Tau [277, 389, 390, 400, 
401]. Tauopathy then spreads systematically from the site of injection to linked brain regions [402-404], implying endocytosis, amplification, transport, and release of Tau seeds between cells [278, 391, 405]. Expression of human Tau in the projection neurons of the entorhinal cortex can subsequently spread to other connected areas [392, 393]. Furthermore, peripheral administration of Tau aggregates can trigger intracerebral tauopathy in transgenic mice [406]. In $\mathrm{AD}$, Tau pathology appears downstream of beta-amyloid aggregation [407, 408]. Additionally, tauopathy is stereotypically distributed through interconnected brain regions, indicating that neuronal trafficking mechanisms contribute to the dissemination of Tau seeds within the CNS $[8,409]$.

Recently, several studies suggested that Tau may adopt distinct conformations and their injection in animal models recapitulated the characteristic hallmark lesions observed in each tauopathy via a prion-like process [278, 389, 399, 410-412].

Despite Tau being a microtubule-associated and cytosolic protein, its release and presence in the extracellular space can be associated with a physiological role. Indeed, neuronal activity can influence Tau release at the synaptic terminal and does not seem connected to a propagation mechanism $[413,414]$. Furthermore, CSF of AD patients display high levels of phosphorylated Tau species that seem to correlate with disease severity [415, 416]. These findings suggest the existence of active cellular processes involved in Tau secretion relevant for its role in disease pathogenesis and progression.

Tau can be secreted and internalized via multiple routes [283, 417]. Release in a free form involves its secretion directly through the plasma membrane [418, 419]. Tau interacts with specific lipids-rich membrane microdomains that allows its penetration and release to the extracellular space facilitated by cell surface heparan sulphate proteoglycans (HSPGs) [420, 421]. Furthermore, this process is partially mediated by interaction with proteins localized in the plasma membrane, such as annexin-A2 and A6 [422]. Misfolded Tau seeds were shown to disrupt membranes by forming pore-like amyloid structures, allowing their release to the extracellular space [423, 424]. Cell-to-cell transfer of Tau seeds can occur via tunnelling nanotubes [425], and the MAPS pathway also promotes the secretion of cytosolic misfolded Tau to the extracellular space $[288,426]$.

Studies have also demonstrated that EVs, in particular ectosomes and exosomes, can incorporate Tau and then fused or be endocytosed by receptor cells [317, 318, 427, 428]. Exosomes isolated from both CSF and brain of AD patients contain Tau [429-432]. Also, injection of these vesicles led to an increase in Tau phosphorylation and formation of inclusions in animal models [433, 434]. Interestingly, Tau release in ectosomes was suggested 
to have a physiological role, while secretion in exosomes may predominate under pathological conditions [317, 318].

After being secreted to the extracellular space, pathological Tau seeds enter in receptor cells through cellular uptake, as dynamin-dependent and receptor-mediated endocytosis [391, 405, 435]. After Tau seeds reach the cytosol of the recipient cells, the templated seeding of the endogenous Tau occurs and leads to further pathology propagation.

\subsubsection{Propagation of huntingtin pathology}

Recently, the observation of $\mathrm{mHtt}$ aggregates within fetal striatal allografts in HD patients supported the possibility of protein spreading mechanisms in monogenic disorders [436]. These clinical pathological observations have been further supported by several in vitro and in vivo studies that demonstrate internalization and transmission of $\mathrm{Htt}$ between cells [279, $280,437-449]$.

To date, the importance of prion-like mechanisms in HD has been questioned due to the genetic origin of the disease and the ubiquitous expression of $\mathrm{Htt}$ throughout the body. However, $\mathrm{Htt}$ is detected in significant quantities in plasma, CSF and extracellular matrix, suggesting that this protein can be exported from the cells of origin [436, 441, 447, 450-454]. Although the precise mechanisms of cell-to-cell transfer are not completely understood, several pathways have been suggested to occur in $\mathrm{HD}$ [282]. Both $\mathrm{Htt}$ and $\mathrm{mHtt}$ can be released to the extracellular space through synaptic transmission, vesicular transport, in a free form, exosomes and tunnelling nanotubes [439-442, 455-458]. More recently, administration of recombinant $\mathrm{mHtt}$ was shown to be taken up by several cell models, as well as to generate disease phenotypes in animal models injected with protein seeds [280].

The transfer of $\mathrm{mHtt}$ between cells through EVs has been a complex and not yet clear mechanism. Interestingly, exosomes have been proposed to be a propagation mechanism for both RNA and $\mathrm{mHtt}$ protein between cells [459]. However, it was also reported the absence of $\mathrm{mHtt}$ in exosomes isolated from platelets of HD patients [460]. Injection of exosomes derived from HD patients in animal models triggered the formation of HD pathology and demonstration of HD-related behaviour [441, 456]. In contrast, exosomes secreted from astrocytes and stem cells have been described to have a neuroprotective role with the reduction of $\mathrm{mHtt}$ aggregates [461, 462].

Once released, secreted $\mathrm{mHtt}$ can be internalized in receptor cells and amplify the disease due to its propensity to seed aggregation of the soluble protein [438, 454, 463]. 
The existence of protein strains and acquisition of sporadic forms of the disease have not been yet addressed in the field. However, there is evidence supporting their potential existence in HD [193, 449, 464]. For example, disease manifestation of patients with an identical CAG repeat length can develop at different ages and follow diverse clinical courses for unknown reasons [197, 465-468]. Allelic variations and environmental factors have been suggested to contribute to the discrepancies in age of onset and disease severity. Furthermore, the existence of different protein strains might explain the high clinical variability within the HD population [282]. These findings highlight a possible role of prion-like mechanisms in HD progression, and the need of further research regarding the factors governing these pathways. 


\subsection{Co-pathology in neurodegenerative diseases}

A relevant topic in neurodegenerative diseases is the co-occurrence of protein inclusions composed of diverse proteins in the same patient, and their participation in the neurodegenerative process [469]. The presence of co-pathology is often accompanied by overlapping clinical symptoms, faster cognitive decline, and shortened lifespan. The correlation between clinical and pathological features in co-pathology has prompted several studies to understand the mechanisms by which diverse pathological protein aggregates accumulate in the brain of patients.

In $A D$, it was observed synergistic effects between beta-amyloid and Tau [470]. Although beta-amyloid is known to originate Tau pathology, beta-amyloid toxicity has been shown to be Tau dependent $[133,149,471]$. Furthermore, Tau levels in the CSF are correlated with disease progression [472, 473]. Also, AD patients exhibit significant LB pathology together with beta-amyloid plaques and NFTs [474-477]. In synucleinopathies, the presence of beta-amyloid plaques is associated with aSyn aggregation and spreading [478, 479].

Tau and aSyn are distinct proteins that contribute to singular disease-specific pathologies, yet several studies suggest that they interact, modulate the aggregation of each other and co-occur in pathological inclusions in the human brain [480]. Tau aggregates have been implicated in PD [396, 481-485]. aSyn enhances Tau phosphorylation, triggering Tau pathogenicity, and these proteins can seed aggregation of each other possibly accelerating the neuropathological cascade [486-490]. Also, recombinant aSyn strains have been shown to promote Tau aggregation [489, 491]. Interestingly, grafted tissue in HD patients exhibited abundant Tau-related pathology [492-495].

These results suggest that co-pathology is a feature in several neurodegenerative diseases. Furthermore, it implies that this phenomenon may be common to several neurodegenerative diseases and a number of proteins may overlap to contribute to the complex pathophysiology of neurodegenerative diseases. Moreover, it suggests the existence of common molecular pathways that bring together singular proteins with very distinctive cellular functions, possibly linking the development of distinct pathological features and resulting in synergistic modes of toxicity. 


\section{Aims of the study}

Several disease-related proteins appear to be transferred from cell-to-cell, contributing for the dissemination of pathology in the brain and disease progression in neurodegenerative diseases. However, the molecular mechanisms involved in the release of these proteins that are typically present in the cytosol are still unclear. Similarly, their effect in neuronal function is also unknown. Therefore, it is important to establish differences and similarities in the ways that disease-related proteins are handled in order to identify specific therapeutic targets for each disease. Hence, we investigated some of the pathways through which cells transfer proteins, but also the consequences of the presence of normal and pathological forms of disease-associated proteins in the extracellular space and after internalization in diverse receptor cell types.

Aim 1 (Publication I). Investigation and characterization of ectosomes and exosomes, assessment of specific protein markers, and functional relevance in neuronal networks in vitro.

Aim 2 (Publication II). Systematic comparison of the molecular mechanisms involved in the release of proteins associated with distinct neurodegenerative disorders, and their consequences in spontaneous neuronal activity. 


\subsection{Publication I}

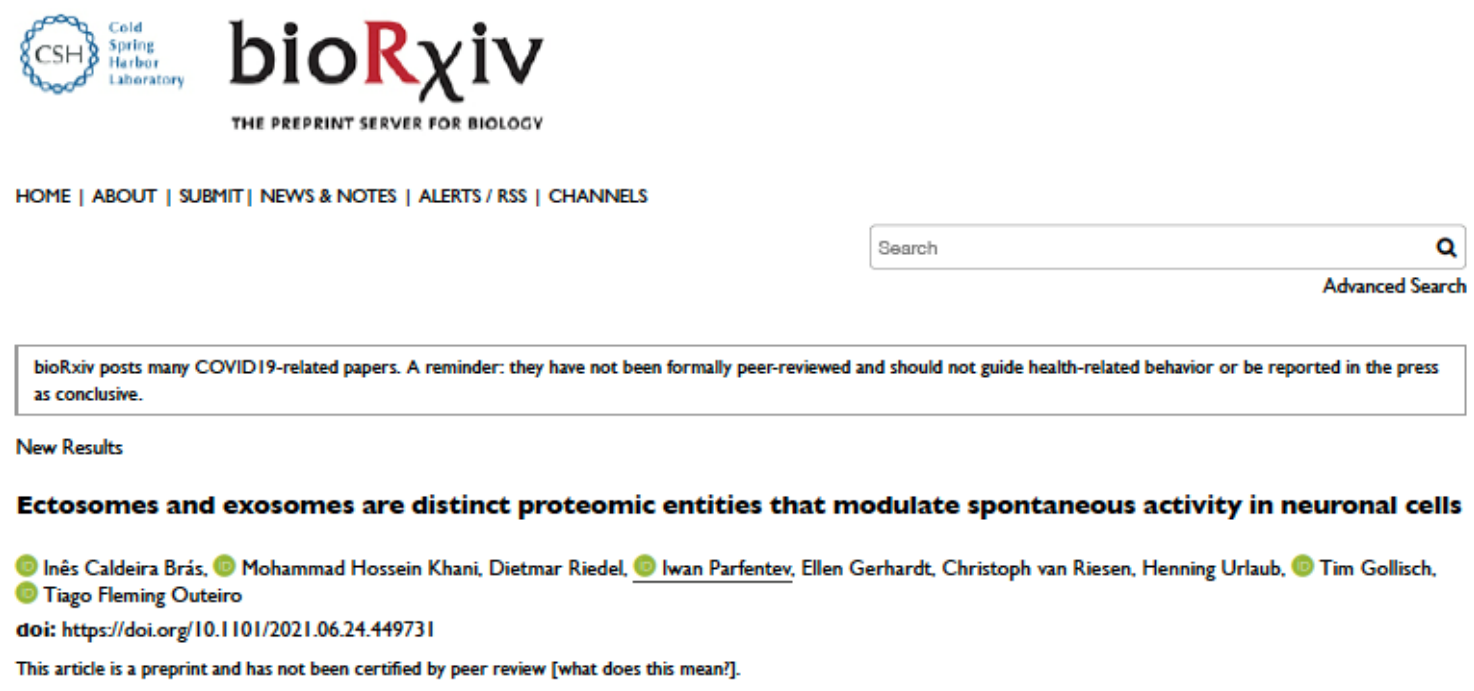

Link: https://www.biorxiv.org/content/10.1101/2021.06.24.449731v1

\section{Author contributions}

Inês Caldeira Brás and Tiago Fleming Outeiro conceived the study. Inês Caldeira Brás performed all the cell culture, molecular biology and imaging experiments. Inês Caldeira Brás and Mohammad Hossein Khani stablished the spike sorting framework, performed multielectrode array experiments and data analysis. Dietmar Riedel performed the electron microscopy experiments. Iwan Parfentev and Henning Urlaub performed the mass spectrometry experiments. Ellen Gerhardt prepared the lentiviral vectors used in the study. Christoph van Riesen provided the cerebrospinal fluid material for the study. Tim Gollisch provided methodology and resources for the multi-electrode array experiments. Inês Caldeira Brás analysed and interpreted the data. Inês Caldeira Brás generated the graphs and figures. Inês Caldeira Brás and Tiago Fleming Outeiro wrote the manuscript. Tiago Fleming Outeiro supervised the work. 


\section{Graphical abstract}

\section{Exosomes}

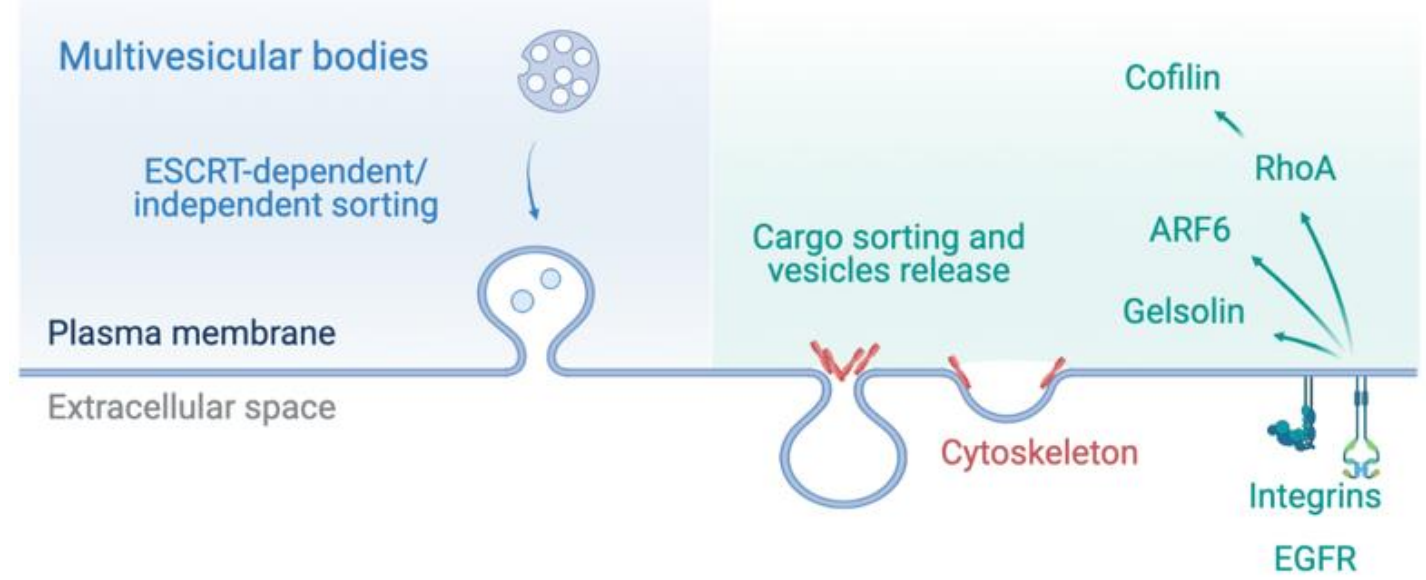

\section{Ectosomes}

EGFR
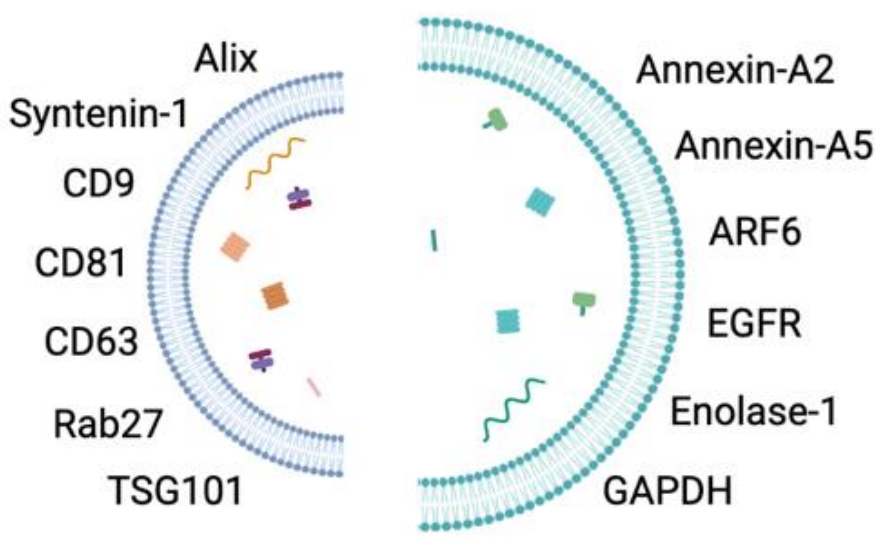


\section{Ectosomes and exosomes are distinct proteomic entities that modulate spontaneous activity in neuronal cells}

Inês Caldeira Brás ${ }^{1}$, Mohammad Hossein Khani², Dietmar Riedel ${ }^{3}$, Iwan Parfentev $^{4}$, Ellen Gerhardt ${ }^{1}$, Christoph van Riesen ${ }^{5,6}$, Henning Urlaub ${ }^{4,7}$, Tim Gollisch², Tiago Fleming Outeiro ${ }^{1,8,9,10,11 \text { * }}$

1Department of Experimental Neurodegeneration, Center for Biostructural Imaging of Neurodegeneration, University Medical Center Göttingen, 37073 Göttingen, Germany.

${ }^{2}$ Department of Ophthalmology, University Medical Center Göttingen, 37073 Göttingen, Germany.

${ }^{3}$ Laboratory of Electron Microscopy, Max Planck Institute for Biophysical Chemistry, 37075 Göttingen, Germany.

${ }^{4}$ Research Group Bioanalytical Mass Spectrometry, Max-Planck-Institute for Biophysical Chemistry, 37077 Göttingen, Germany.

${ }^{5}$ Department of Neurology, University Medical Center Göttingen, 37075 Göttingen, Germany. ${ }^{6}$ German Center for Neurodegenerative Diseases (DZNE), 53127 Bonn, Germany.

${ }^{7}$ Bioanalytics, Institute of Clinical Chemistry, University Medical Center Göttingen, 37075 Göttingen, Germany.

${ }^{8}$ Max Planck Institute for Experimental Medicine, 37075 Göttingen, Germany.

${ }^{9}$ Translational and Clinical Research Institute, Faculty of Medical Sciences, Newcastle University, NE2 4HH, United Kingdom.

${ }^{10}$ Scientific employee with an honorary contract at German Center for Neurodegenerative Diseases (DZNE), 37075 Göttingen, Germany.

"Correspondence: touteir@gwdg.de

${ }^{11}$ Lead contact:

Prof. Dr. Tiago Fleming Outeiro

Department of Experimental Neurodegeneration, University Medical Center Göttingen, 37073

Göttingen, Germany

E-mail: touteir@gwdg.de

Office Phone: $+49(0) 5513913544$

Fax: $+49(0) 5513922693$ 


\begin{abstract}
Extracellular vesicles (EVs) are important mediators in intercellular communication. However, understanding the biological origin and functional effects of EVs subtypes has been challenging due to the moderate differences in their physical properties and absence of reliable markers. Here, we characterize the proteomes of ectosomes and exosomes using an improved differential ultracentrifugation protocol and quantitative proteomics. Cytoskeleton and glycolytic proteins are distinctively present in ectosomes, while endosomal sorting complexes proteins and tetraspanins are enriched in exosomes. Furthermore, annexin-A2 was identified as a specific marker for ectosomes derived from cell media and human cerebrospinal fluid. Expression of EGFP as a cytosolic reporter leads to its incorporation in EVs and enables their imaging with higher resolution. Importantly, ectosomes and exosomes internalization in neuronal cells results in the modulation of neuronal spontaneous activity. Our findings suggest that EVs cargoes reflect core intracellular processes, and their functional properties might regulate basic biological and pathological processes.
\end{abstract}

Keywords: Extracellular vesicles, ectosomes, microvesicles, exosomes, spreading, proteomics, multi-electrode array, neuronal activity 


\section{Introduction}

Extracellular vesicles (EVs) are important vehicles in intercellular communication, mediating long range signalling events [293-296]. These vesicles are important in the central nervous system (CNS), where they are secreted by diverse cell types, appearing also in the cerebrospinal fluid (CSF) [297, 298]. Proteins, RNA, and lipids are actively and selectively incorporated into EVs, justifying the importance of these vesicles not only in normal biology but also in disease, as they may report on pathological alterations [299-301].

Size and mechanisms of biogenesis are the conventional classification approaches for EVs [302, 303, 310]. Exosomes (30 -150nm in diameter) are derived from endosomes released from multivesicular bodies (MVBs) after fusion with the plasma membrane [305, 306]. In contrast, microvesicles (also termed ectosomes, $100-1000 \mathrm{~nm}$ in diameter) are larger EVs generated by direct shedding from the plasma membrane [307, 308].

While exosomes have been characterized in multiple studies, ectosomes remain understudied. Furthermore, disparities in purification strategies and lack of reliable protein markers that can discriminate between these EVs types limits our knowledge regarding ectosomes [295, 299, 309]. Therefore, further characterization of ectosomes may provide valuable information on biogenesis, cargo sorting and functional roles of these vesicles in physiological and pathological conditions.

Herein, we provide an in-depth molecular and functional characterization of ectosomes and exosomes based on differential ultracentrifugation. Comprehensive proteomic analysis revealed specific protein composition and pathway enrichment for each EV subtype. Exosomes are composed of endosomal sorting proteins required for transport (ESCRT) and tetraspanins [496]. In contrast, ectosomes are enriched in cytoskeletal proteins, glycolytic enzymes, integrins and annexins. Interestingly, ectosomes isolated from human CSF and from cell media are enriched in annexin-A2, suggesting this protein can be exploited as an important marker for ectosomes characterization. EGFP incorporation in both ectosomes and exosomes enabled their imaging at higher resolution when compared with the use of the thiolbased dye Alexa Fluor 633 C5-maleimide. Remarkably, we demonstrate that EVs internalization affects the spontaneous activity of primary cortical neurons. Our work provides novel insight into the cell biology of intercellular communication via EVs, demonstrating they transfer cargoes that can modulate cellular function. Ultimately, our study also forms the foundation for future biomarker studies and for the understanding of the molecular basis of different diseases. 


\section{Results}

\section{Separation of ectosomes and exosomes by differential ultracentrifugation}

Biological samples contain a heterogeneous mixture of EVs. To understand their composition and functional properties, ectosomes and exosomes were isolated from the media of human HEK cells using an improved differential ultracentrifugation protocol (Figure 1A) [317, 497]. To avoid possible contamination with EVs from fetal bovine serum (FBS) present in the cell media, cells were incubated with conditioned media (previously depleted of EVs) for 24 hours, as previously described [497, 498].

A

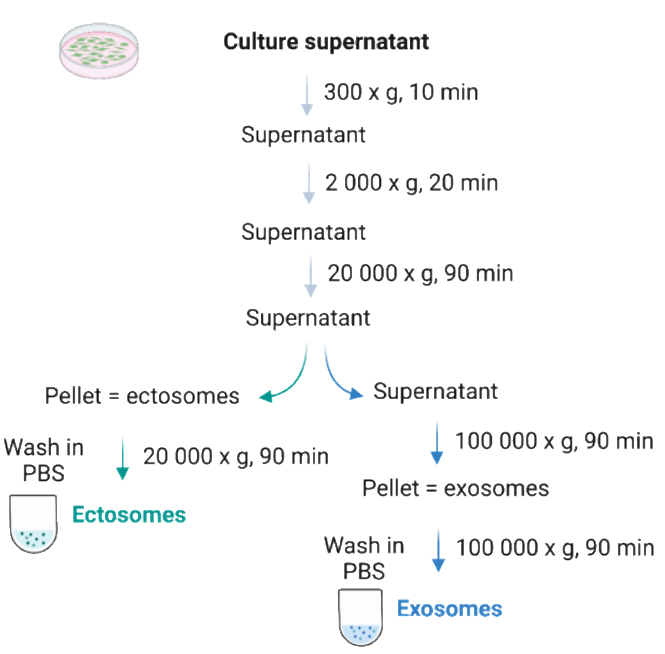

Label-free quantitative Biochemical characterisation mass spectrometry

Biochemical characterisation
of ectosomes and exosomes

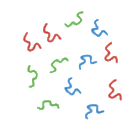

In vitro studies

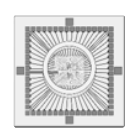

B
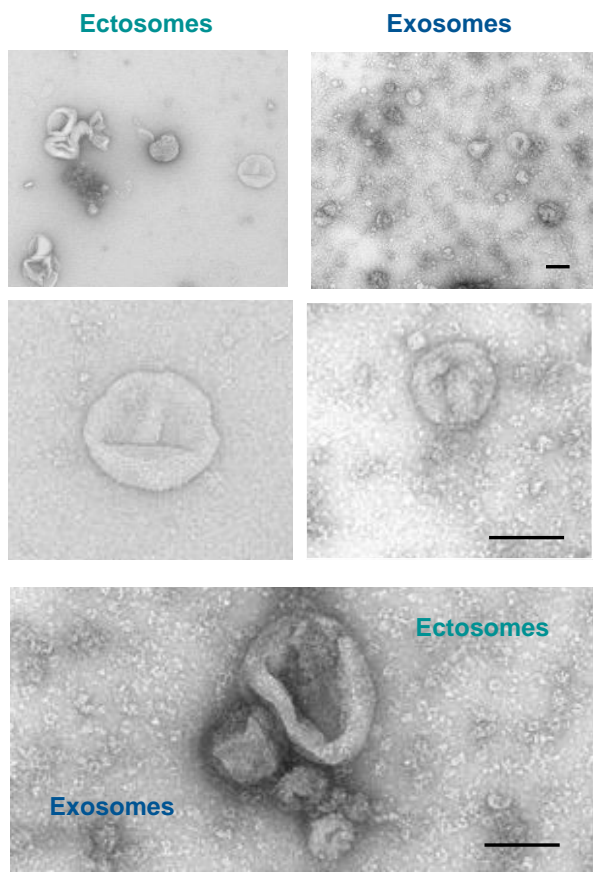

C
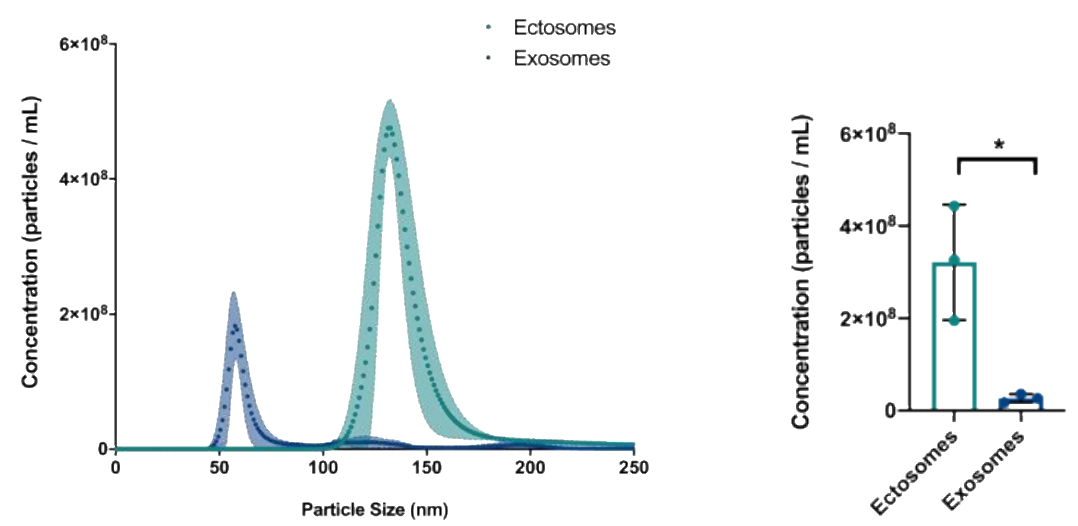

Figure 1. Purification and characterization of secreted EVs using differential centrifugation. (A) Schematic overview of the EVs purification protocol. Conditioned cell media was collected from HEK cells after 24 hours and subsequently centrifuged at different speeds. After isolation, EVs were used for label-free quantitative mass spectrometry, immunoblot and MEA recordings. (B) Whole-mount EM analysis of each pellet showing 
representative images of ectosomes and exosomes (scale bar 100nm). (C) NTA measurements of particle concentrations and average size distributions of ectosomes and exosomes. Data from at least three independent experiments. Significant differences were assessed by two-tailed unpaired t-test comparison and are expressed as mean $\pm S D,{ }^{*} p<0.05$.

Both EVs subtypes exhibited a cap-shaped morphology, typical for negative stained vesicular structures, and presented the expected size differences by electron microscopy (EM) (Figure 1B). Nanoparticle tracking analysis (NTA) was used to determine the size and to quantity of ectosomes and exosomes based on Brownian motion (Figure 1C). The diameter distribution for ectosomes was considerably larger and peaked at 140nm, while exosomes had an average diameter of 60nm (Figure 1C). Furthermore, the concentration of ectosomes was significantly greater when compared to exosomes (Figure 1C).

\section{Ectosomes and exosomes exhibit characteristic proteomic profiles}

To determine the protein composition of ectosomes and exosomes, label-free quantitative mass spectrometry was performed (Figure 2). In total, 2216 proteins were identified in our study, with 371 proteins exclusively recognized in ectosomes, and 193 proteins enriched in exosomes (Figure 2A, Supplementary Tables 1 and 2).

A

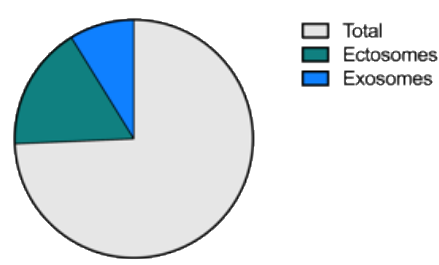

Total number of proteins identified $\mathbf{2} \mathbf{2 2 1 6}$ Number of proteins enriched in ectosomes $=371$ Number of proteins enriched in exosomes $=193$
$=193$

B

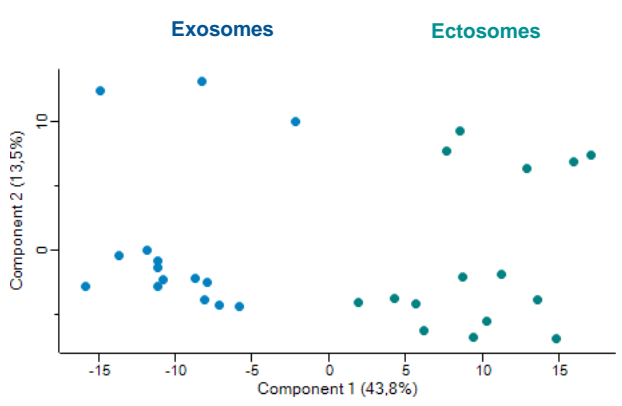

C
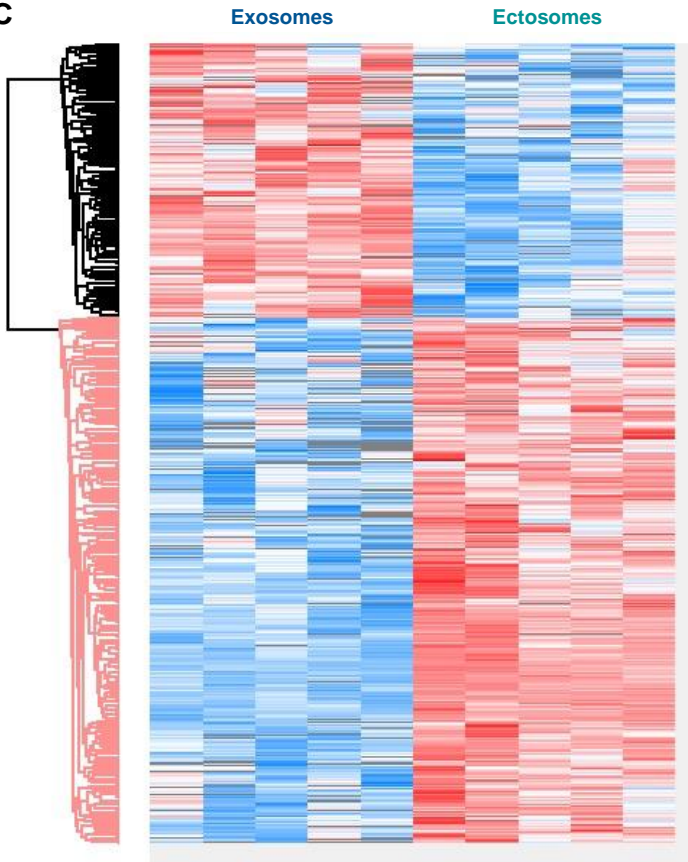

Figure 2. Proteomic analyses of ectosomes and exosomes using label-free quantitative mass spectrometry. (A) Diagram representing the number of unique proteins identified in each EV type and total number of proteins identified in the study. (B) PCA of the quantitative differences in spectral counts between ectosomes (turquoise) and exosomes (blue) in the biological replicates. (C) Heatmap of the significantly different identified 
proteins from proteomic profiling of in ectosomes and exosomes. High and low expression are shown in red and blue, respectively (protein intensity z-score -2 for dark blue, protein intensity z-score for 2 dark red). Data represented from five independent samples. Data analyses were performed using Perseus software.

Principal component analysis (PCA) of protein composition revealed a clear separation of the two EVs subtypes (Figure 2B). Despite some overlap in identifiable proteins, which was to be expected, hierarchical clustering of the protein datasets uncovered two separate fractions with distinct proteomic profiles (Figure 2C).

These results indicate individual proteomic profiles of ectosomes and exosomes, supporting functional biological differences between the two EVs subtypes.

\section{Exosomes are enriched with tetraspanins and ESCRT-related proteins, while ectosomes contain cytoskeletal proteins and glycolytic enzymes}

Next, we searched for specific markers of exosomes and ectosomes among the proteins uniquely present in each EV subtype [310]. Proteomic profiling identified a number of known exosomal proteins, including ESCRT and vacuolar protein sorting-associated (VPS) proteins (Supplementary Table 1). This included alix (PDCD6IP), syntenin-1 (SDCBP), vesicle-associated membrane protein 2 (VAMP2) and VPS25/28/37B (VPS25/28/37B) (Figure 3A, Table 1). Since the ESCRT machinery is important for the sorting of ubiquitinated cargo and regulation of exosomal biogenesis, their identification in our exosomal fraction is consistent with previous studies [496, 497, 499-501]. Tetraspanins are highly enriched in exosomes and are associated with exosome biogenesis [502]. Consistently, our exosomal fraction comprised CD63 antigen (CD63), CD81 antigen (CD81) and tetraspanin-4, 6, 7, 9 (TSPAN4/6/ 7/9) (Figure 3B, Supplementary Table 1). Furthermore, our proteomic analysis also highlighted the presence of lipid raft components in exosomes, such as flotillin-1 and 2 (FLOT1/ 2). However, their levels were not statistically different from the ones found in ectosomes. Similarly, TSG101 (TSG101) and CD9 (CD9) were also slightly enriched in exosomes, although this did not reach statistical significance in comparison to the levels found in ectosomes. Importantly, our results indicate that certain proteins commonly considered exosomal can also occur in ectosomes, emphasizing the need for using multiple protein markers to distinguish EVs.

In our study, we identified 11 proteins in exosomes that are frequently identified in EVs, of the 100 most frequently reported proteins in Vesiclepedia and ExoCarta databases [503, 504] (Table 2, Supplementary Table 3). This indicates the specificity in proteins in exosomes and the existence of common exosomal protein markers across different samples. 
Table 1. List of top 30 up-regulated proteins in exosomes identified in this study organized according to the significance values (full list in Supplementary Table 1).

\begin{tabular}{|c|c|c|c|}
\hline Protein names & Gene names & Biological process & $\begin{array}{l}-\log _{10}(p- \\
\text { value })\end{array}$ \\
\hline Alix & $P D C D 6 I P$ & Cell cycle & 4,836 \\
\hline Glycogen phosphorylase & $P Y G M / L$ & Alcohol metabolic process & 4,300 \\
\hline Proteasome subunit alpha/ beta & $P S M A / B$ & Protein catabolic process & 4,098 \\
\hline Asporin & $A S P N$ & Anatomical structure development & 3,734 \\
\hline Homogentisate 1,2-dioxygenase & $H G D$ & Amine metabolic process & 3,611 \\
\hline Receptor-type tyrosine-protein phosphatase & PTPRM & Axon guidance & 3,610 \\
\hline $\begin{array}{c}\begin{array}{c}\text { Vacuolar protein sorting-associated protein } 28 \\
\text { homolog }\end{array} \\
\end{array}$ & VPS28 & Catabolic process & 3,525 \\
\hline Cation-independent mannose-6-phosphate receptor & IGF2R & $\begin{array}{c}\text { Anatomical structure } \\
\text { morphogenesis }\end{array}$ & 3,377 \\
\hline Eukaryotic initiation factor $4 \mathrm{~A}-\mathrm{II}$ & EIF4A2 & Biological regulation & 3,345 \\
\hline Protein kinase C-binding protein NELL2 & NELL2 & Cellular homeostasis & 3,340 \\
\hline 3-mercaptopyruvate sulfurtransferase & MPST & Biosynthetic process & 3,327 \\
\hline Mannose-1-phosphate guanyltransferase beta & GMPPB & Alcohol biosynthetic process & 3,264 \\
\hline Cytosolic 10-formyltetrahydrofolate dehydrogenase & ALDH1L1 & $\begin{array}{c}10 \text {-formyltetrahydrofolate catabolic } \\
\text { process }\end{array}$ & 3,226 \\
\hline Cytoplasmic aconitate hydratase & ACO1 & Acetyl-CoA catabolic process & 3,172 \\
\hline S-adenosylmethionine synthase isoform type-1 & MAT1A & Amine biosynthetic process & 3,097 \\
\hline Delta-aminolevulinic acid dehydratase & $A L A D$ & Biosynthetic process & 3,039 \\
\hline Tubulin alpha-4A chain & TUBA4A & Cell cycle & 3,033 \\
\hline Vacuolar protein sorting-associated protein 37B & VPS37B & Cellular component assembly & 3,012 \\
\hline Adenosylhomocysteinase & $A H C Y$ & Amine metabolic process & 2,989 \\
\hline Regucalcin & $R G N$ & Calcium ion homeostasis & 2,989 \\
\hline Fructose-bisphosphate aldolase B & $A L D O B$ & Alcohol biosynthetic process & 2,989 \\
\hline 4-trimethylaminobutyraldehyde dehydrogenase & ALDH9A1 & Amine biosynthetic process & 2,960 \\
\hline $\begin{array}{l}\text { Microtubule-associated protein RP/EB family member } \\
2\end{array}$ & MAPRE2 & Cell cycle & 2,933 \\
\hline Uroporphyrinogen decarboxylase & $U R O D$ & Cellular metabolic process & 2,927 \\
\hline Coronin-1A & CORO1A & Actin cytoskeleton organization & 2,918 \\
\hline Haptoglobin & $H P$ & Inflammatory response & 2,859 \\
\hline Collagen alpha-3(VI) chain & COL6A3 & Axon guidance & 2,814 \\
\hline Argininosuccinate synthase & ASS1 & Amide biosynthetic process & 2,791 \\
\hline Galectin-3-binding protein & LGALS3BP & Biological adhesion & 2,774 \\
\hline von Willebrand factor & VWF & Biological adhesion & 2,736 \\
\hline
\end{tabular}

Table 2. Top 11 proteins identified in this study and present in the Top 100 proteins list of often identified in EVs (in total our study found 11 proteins enriched in exosomes that are referred in the Top 100 list in vesiclepedia (Supplementary Table 3).

\begin{tabular}{|c|c|c|c|}
\hline Protein names & Gene names & Biological process & $\begin{array}{c}\text { - } \text {-og }_{10}(\mathbf{p}- \\
\text { value })\end{array}$ \\
\hline Alix & $P D C D 6 I P$ & Cell cycle & 4,836 \\
\hline Adenosylhomocysteinase & $A H C Y$ & Amine metabolic process & 2,989 \\
\hline Galectin-3-binding protein & $L G A L S 3 B P$ & Biological adhesion & 2,774 \\
\hline Syntenin-1 & $S D C B P$ & Actin cytoskeleton organization & 2,464 \\
\hline GTP-binding nuclear protein Ran & $R A N$ & Actin cytoskeleton organization & 1,877 \\
\hline CD63 antigen & $C D 63$ & Biological adhesion & 1,589 \\
\hline Talin-1 & $T L N 1$ & Actin cytoskeleton organization & 1,479 \\
\hline Gelsolin & $G S N$ & Actin cytoskeleton organization & 1,477 \\
\hline CD81 antigen & $C D 81$ & Activation of MAPK activity & 1,454 \\
\hline Complement C3 & $C 3$ & Activation of immune response & 1,344 \\
\hline Alpha-2-macroglobulin & $A 2 M$ & Biological regulation & 1,112 \\
\hline
\end{tabular}


In ectosomes, we detected several proteins commonly identified in larger vesicles, such as plexin-A1 (PLXA1), glutathione S-transferase (GSTP1), the plasma membrane marker sodium/potassium-transporting ATPase (ATP1A1) and epidermal growth factor receptor (EGFR) (Figure 3C, Table 3 and Supplementary Table 2) [299, 309, 500]. Importantly, ADP-ribosylation factor $6(A R F 6)$ is thought to regulate ectosome biogenesis, and was also identified in our dataset (Figure 3C) [505].

Actin, tubulin, and keratins are highly abundant cellular cytoskeletal proteins observed in EVs preparations [305, 499, 500]. In our proteomic dataset for ectosomes, we found an enrichment in cytoskeletal proteins as profilin-1 (PFN1), cofilin-1/ 2 (CFL1/2), vimentin (VIM), ezrin (EZR), moesin (MSN), tubulin beta chain (TUBB) and actin (ACTG1) (Figure 3D, Table 3 and Supplementary Table 2). The absence of other cytoskeletal-associated proteins in our ectosomes and exosomes, such as those involved in actin filament polymerization, associated with microtubules, or intermediate filaments, may be related with their exclusion as potential contaminants in our hit list using Perseus software. The removal of these proteins avoided the identification of proteins that might be present in the cell media but not specifically in our vesicle fractions (Supplementary Table 1 and 2).

Interestingly, our analyses also revealed a significant enrichment in metabolic enzymes in ectosomes, such as peroxiredoxin-1/2/ 6 (PRDX1/2/6), pyruvate kinase (PKM), alpha-enolase (ENO1) and glyceraldehyde-3-phosphate dehydrogenase (GAPDH) (Figure 3E, Supplementary Table 2). The presence of annexin family members in EVs fractions has been previously documented and, importantly, perturbations in the levels of their secretion are associated with disease [506]. Remarkably, we found several annexin proteins enriched in ectosomes, including annexin-A1, A2, A3, A4, A5, A6, A7, A8, A11 (ANXA1/ 2/ 3/ 4/ 5/ 6/ 7) 8/ 11) (Figure 3F, Table 3 and Supplementary Table 2). Integrins have been reported in microvesicles $[505,507]$ and we found them abundantly in ectosomes [alpha-1/ 2/ 5/ 6 (ITGA1) 2/5/6)] (Supplementary Figure 1A, Supplementary Table 2). Furthermore, other proteins were enriched in ectosomes as clathrin $(C L T C)$, MHC class I $(H L A-A / B)$, cell division control protein 42 (CDC42), 14-3-3 proteins (YWHAZ/ E/ B/ G/ H/ Q), histones H1.4 / H1.0/ H2A (HIST1H1E / H1FO/ HIST2H2AC), heat shock protein HSP90 (HSP90AB1/ AA1), heat shock $70 \mathrm{kDa}$ protein (HSPA1A B, HSPA4) and heat shock protein $105 \mathrm{kDa}$ (HSPH1) (Supplementary Figure 1A, Supplementary Table 2). Matrix metalloproteinase 2 (MMP2) is a protease in the extracellular matrix and it was previously described as an ectosomal marker [508, 509]. However, in our study we did not find an enrichment in matrix metalloproteinases proteins (Supplementary Table 2).

Rabs are small GTPases that regulate numerous vesicle docking and fusion events, including the sorting and trafficking of MVBs to the plasma membrane [295]. Rabs form 
complexes with proteins involved in membrane trafficking through the endocytic system and are usually used as markers of different endocytic compartments. Despite being frequently identified in exosomes, we also identified several Rab proteins in ectosomes (Supplementary Figure 1B, Supplementary Tables 1 and 2). In particular, Rab-1A/ 1B/4A/5B/27B (RAB1A $1 B / 4 A / 5 B / 27 B$ ) were enriched in exosomes, while Rab-2A/5C/6A/6B/7A/ 8A/ 8B/ 9A/ 10/ 11B/ 13/21/ 23/ 35 (RAB2A/5C/6A/6B/7A/8A/ 8B/9A/ 10/11B/ 13/21/23/35) were present in ectosomes (Supplementary Figure 1B, Supplementary Tables 1-2). Our findings suggest that Rab proteins may play unique roles in the biogenesis of distinct types of EVs.

A

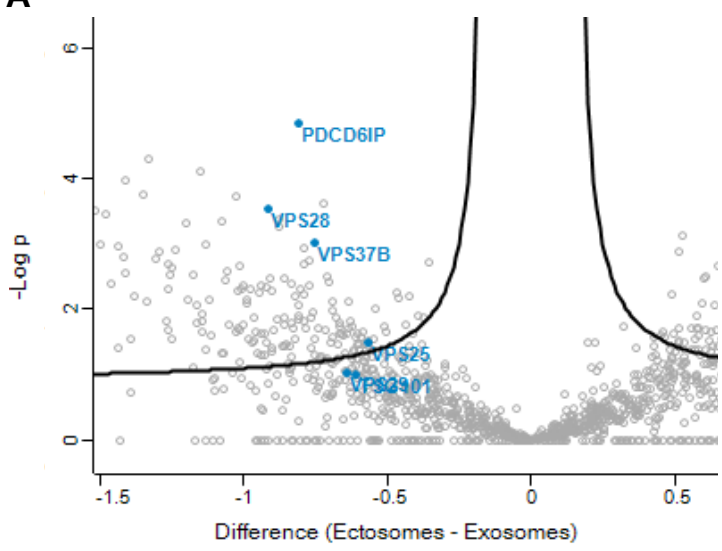

B

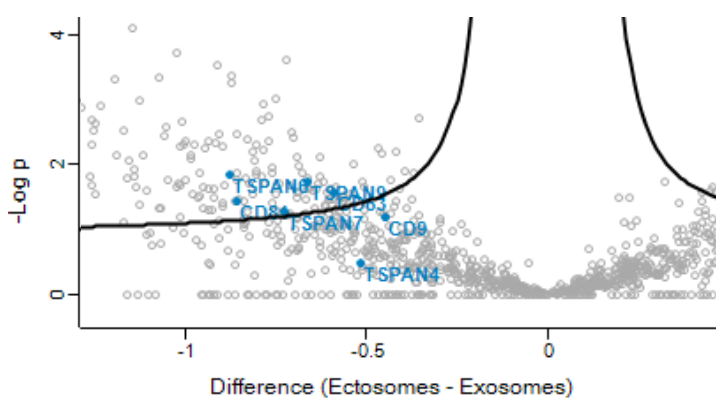

C

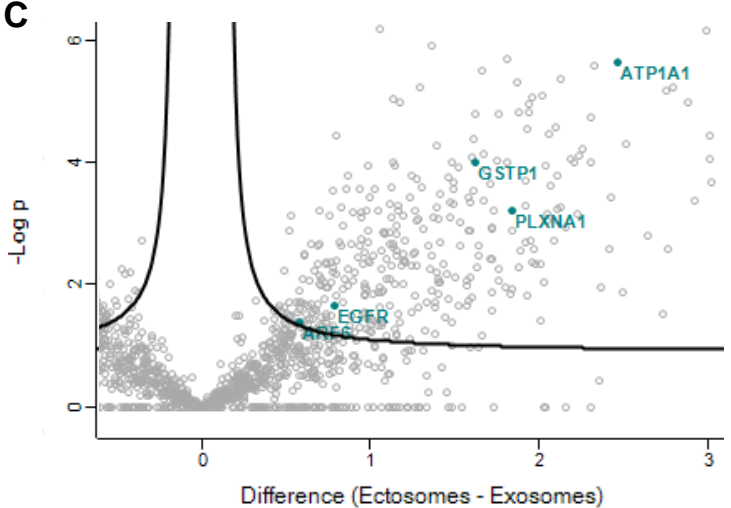

D

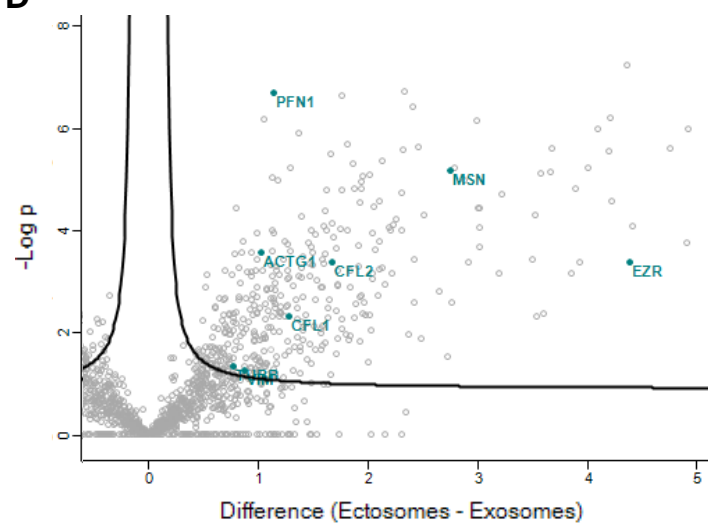

E

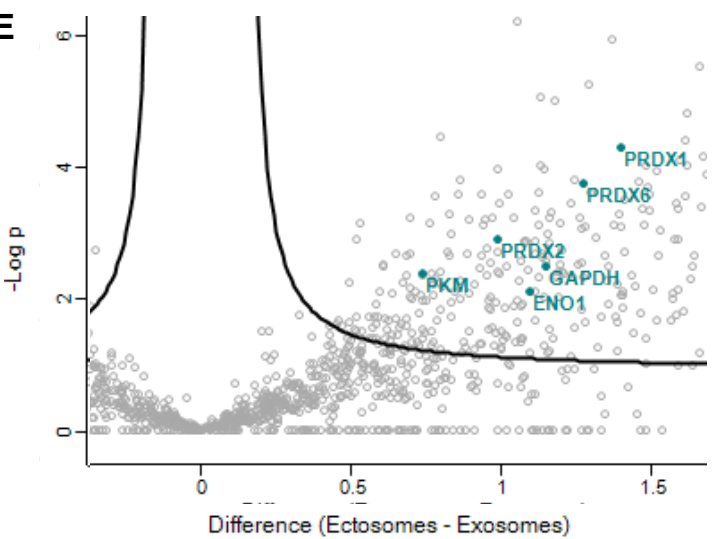

$\mathbf{F}$

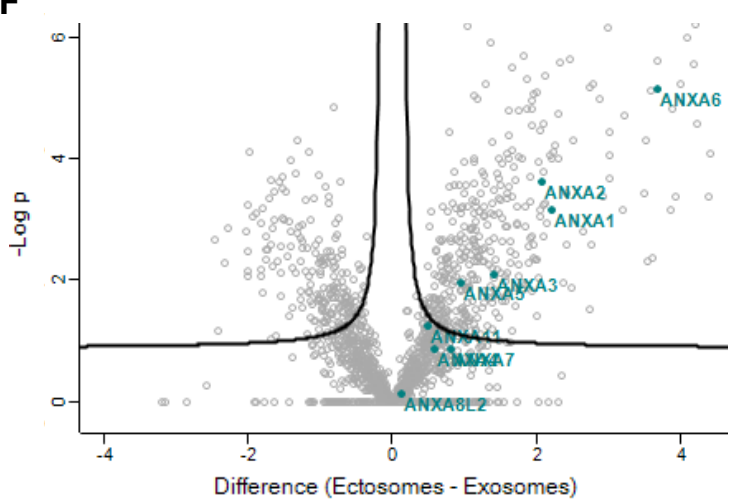

Figure 3. Protein hits enriched in ectosomes and exosomes. Volcano plots of quantitative differences in proteins in EVs fractions. (A) Endosomal sorting complexes required for transport (ESCRT) proteins present in 
exosomes - alix (PDCD6IP), tumor susceptibility 101 (TSG101) and vacuolar protein sorting-associated proteins 25/28/29/37B (VPS25/28/29/37B). (B) Tetraspanins proteins enriched in exosomes - CD9 antigen (CD9), CD63 antigen (CD63), CD81 antigen (CD81) and tetraspanin-4, 6, 7, 9 (TSPAN4/6/ 7/ 9). (C) Commonly identified proteins in larger vesicles, as ectosomes - plexin-A1 (PLXA1), glutathione S-transferase (GSTP1), sodium/potassium-transporting ATPase (ATP1A1), ADP-ribosylation factor 6 (ARF6) and Epidermal growth factor receptor (EGFR). (D) Cytoskeletal proteins enriched in ectosomes - profilin-1 (PFN1), cofilin-1/ 2 (CFL1/ 2), vimentin (VIM), ezrin (EZR), moesin (MSN) tubulin beta chain (TUBB) and actin (ACTG1). (E) Metabolic enzymes identified in ectosomes - peroxiredoxin-1/2/ 6 (PRDX1/2/6), pyruvate kinase (PKM), alpha-enolase (ENO1) and glyceraldehyde-3-phosphate dehydrogenase (GAPDH). (F) Annexin proteins enriched in ectosomes - annexin A1, A2, A3, A4, A5, A6, A7, A8, A11 ( $A N X A 1 / 2 / 3 / 4 / 5 / 6 / 7 / 8 / 11)$. Blue dots represent the proteins enrichment in exosomes, while turquoise dots represent enrichment in ectosomes. Proteins in the plots are identified with their gene name. Dots above the dashed line represent proteins for which differences were significant (false discovery rate $[F D R]<0.1)$. Data represented in "t-test Difference (Ectosomes - Exosomes)" vs. "-Log t-test p-value" from five independent samples for each group. Data analyses were performed using Perseus software. See also Supplementary Figure 1.

Table 3. List of top 30 up-regulated proteins in ectosomes identified in this study organized according to the significance values (full list in Supplementary Table 2).

\begin{tabular}{|c|c|c|c|}
\hline Protein names & Gene names & Biological process & $-\log _{10}(p-v a l u e)$ \\
\hline Band 4.1-like protein 2/ 3 & $E P B 41 L 2 / L 3$ & Actin cytoskeleton organization & 9,852 \\
\hline Plasma membrane calcium-transporting ATPase 1 & ATP2B1 & Ion transmembrane transport & 7,567 \\
\hline Phosphatidylinositol 4-kinase alpha & $P / 4 K A$ & Actin cytoskeleton organization & 7,239 \\
\hline Tubulin-specific chaperone A & $T B C A$ & Cellular component assembly & 6,731 \\
\hline Profilin-1 & PFN1 & Actin cytoskeleton organization & 6,708 \\
\hline MARCKS-related protein & MARCKSL1 & Regulation of cell proliferation & 6,644 \\
\hline 4F2 cell-surface antigen heavy chain & $S L C 3 A 2$ & Amine transport & 6,412 \\
\hline Cytoplasmic FMR1-interacting protein 1 & CYFIP1 & Axon extension & 6,209 \\
\hline Myelin protein zero-like protein 1 & $M P Z L 1$ & Cell communication & 6,167 \\
\hline $\mathrm{Na}(+) / \mathrm{H}(+)$ exchange regulatory cofactor NHE-RF1 & SLC9A3R1 & Actin cytoskeleton organization & 6,151 \\
\hline Unconventional myosin-lc & MYO1C & Cellular component organization & 5,986 \\
\hline Protein scribble homolog & SCRIB & Anatomical structure development & 5,985 \\
\hline $\mathrm{N}(\mathrm{G}), \mathrm{N}(\mathrm{G})$-dimethylarginine dimethylaminohydrolase 1 & $D D A H 1$ & Amine catabolic process & 5,900 \\
\hline 2,3-cyclic-nucleotide 3-phosphodiesterase & CNP & Aging, synaptic transmission & 5,696 \\
\hline Sodium/potassium-transporting ATPase subunit alpha-1 & ATP1A1 & Cation homeostasis & 5,640 \\
\hline FERM, RhoGEF and pleckstrin domain-containing protein 1 & FARP1 & Actin cytoskeleton organization & 5,604 \\
\hline Integrin alpha-5 & ITGA5 & Cell projection organization & 5,589 \\
\hline Catenin delta-1 & CTNND1 & Axon guidance & 5,556 \\
\hline Charged multivesicular body protein 6 & CHMP6 & Adherens junction organization & 5,507 \\
\hline Basigin & $B S G$ & Cellular component organization & 5,349 \\
\hline PDZ domain-containing protein GIPC1 & GIPC1 & $\begin{array}{c}\text { Anatomical structure } \\
\text { morphogenesis }\end{array}$ & 5,304 \\
\hline Plastin-3 & PLS3 & Cell communication & 5,244 \\
\hline Peripheral plasma membrane protein CASK & CASK & Anatomical structure development & 5,235 \\
\hline Trifunctional purine biosynthetic protein adenosine- 3 & GART & $\begin{array}{c}\text { Calcium ion transmembrane } \\
\text { transport }\end{array}$ & 5,214 \\
\hline 14-3-3 protein epsilon & YWHAE & Protein binding & 5,213 \\
\hline Moesin & MSN & Cell migration & 5,169 \\
\hline Annexin-A6 & ANXA6 & Calcium ion binding & 5,150 \\
\hline Myristoylated alanine-rich C-kinase substrate & MARCKS & Calcium ion transport & 5,105 \\
\hline Guanine nucleotide-binding protein subunit alpha-13 & GNA13 & Activation of adenylate cyclase & 5,054 \\
\hline Ubiquitin-40S ribosomal protein S27a & $R P S 27 A$ & Activation of MAPK activity & 5,027 \\
\hline
\end{tabular}


We also assessed if the proteins we identified in ectosomes are among the 100 most commonly identified EVs proteins, reported in the Vesiclepedia and ExoCarta databases (Supplementary Table 2 and 3) [503, 510]. Interestingly, we found 52 proteins in ectosomes that are common to this list (Table 4, Supplementary Table 2). Strikingly, cytoskeleton proteins and cytosolic glycolytic enzymes were absent from our exosomal fraction and were only identified in ectosomes (Supplementary Table 2).

In summary, proteomic profiling revealed a diverse protein content in both ectosomes and exosomes but enabled us to identify a unique protein signature that enables their distinction.

Table 4. Top 20 proteins identified in this study and present in the Top 100 proteins list of often identified in EVs (in total our study found 52 proteins enriched in ectosomes that are referred in the Top 100 list in vesiclepedia (Supplementary Table 3).

\begin{tabular}{|c|c|c|c|}
\hline Protein names & Gene names & Biological process & $-\log _{10}(p$-value) \\
\hline Profilin-1 & PFN1 & Actin cytoskeleton organization & 6,708 \\
\hline 4F2 cell-surface antigen heavy chain & SLC3A2 & Amine transport & 6,412 \\
\hline Sodium/potassium-transporting ATPase subunit alpha-1 & ATP1A1 & Cation homeostasis & 5,640 \\
\hline 14-3-3 protein epsilon, beta/alpha, theta, zeta/delta & $Y W H A E / A B / Q / Z$ & Amine metabolic process & 5,213 \\
\hline Moesin & $M S N$ & Cell migration & 5,169 \\
\hline Annexin-A6 & ANXA6 & Calcium ion binding & 5,150 \\
\hline Peroxiredoxin-1 & $P R D X 1$ & Anatomical structure homeostasis & 4,263 \\
\hline Fatty acid synthase & FASN & Acetyl-CoA metabolic process & 4,138 \\
\hline Guanine nucleotide-binding protein $\mathrm{G}$ & GNAS & Activation of adenylate cyclase & 4,055 \\
\hline Ras GTPase-activating-like protein & IQGAP1 & Biological regulation & 3,751 \\
\hline Annexin-A2 & ANXA2 & Calcium binding & 3,628 \\
\hline Integrin beta-1 & ITGB1 & Actin cytoskeleton organization & 3,579 \\
\hline Ezrin & $E Z R$ & Actin cytoskeleton organization & 3,375 \\
\hline Heat shock protein HSP 90-beta & HSP90AB1 & Cellular response to stress & 3,294 \\
\hline Ras-related protein Rab-10 & $R A B 10$ & $\begin{array}{c}\text { Anatomical structure } \\
\text { morphogenesis }\end{array}$ & 3,255 \\
\hline T-complex protein 1 subunit alpha & TCP1 & Cell recognition & 3,167 \\
\hline Annexin-A1 & ANXA1 & Innate immune response & 3,162 \\
\hline Ras-related protein Rab-7a & $R A B 7 A$ & $\begin{array}{l}\text { Antigen processing and } \\
\text { presentation }\end{array}$ & 3,137 \\
\hline L-lactate dehydrogenase $\mathrm{A}$ chain & $L D H A$ & Alcohol metabolic process & 2,965 \\
\hline Elongation factor 1 -alpha 1 & EEF1A1 & Biosynthetic process & 2,761 \\
\hline
\end{tabular}

\section{Gene ontology enrichment terms are unique for ectosomes and exosomes}

To further understand the distinct biological roles of exosomes and ectosomes, we performed (GO) enrichment analysis using Perseus software (Figures 4 and 5). Signalling processes, immune response, as well as proteasomal and ubiquitination biological processes were enriched in exosomes (Figures 4A). Furthermore, most of the proteins identified were associated with proteasome, organelle, and vesicle cellular components, correlating with cytosolic molecular functions of these proteins (Figure 4B and C). Using STRING, we next assessed the physical and functional protein association networks, in order to identify known 
and predicted protein-protein interactions [511, 512]. These analyses exhibited a cluster of protein-protein interactions involving proteasomal proteins, as highlighted in the KEGG pathway analyses (Figure 4D and E, Supplementary Figure 2).

In ectosomes, several of the enriched biological processes were related with the regulation of biological and cellular processes, ion transport, actin regulation related pathways and signal transduction (Figure 5A). As expected, our molecular function hits included protein binding, enzymes, and cytoskeletal proteins (Figure 5B). Also, we found a significant enrichment of both glycolysis and gluconeogenesis, key processes of energy metabolism. A large fraction of the proteins identified were associated with plasma membrane, vesicles, cytoplasm and cell interaction and communication processes, thus enabling ectosomes to act as putative intercellular transporters (Figure 5C). Furthermore, KEGG pathway analyses revealed an enrichment of ectosomes in actin cytoskeleton processes and tight junctions, compatible with their plasma membrane origin and enrichment in membrane and cytoplasmic proteins (Figure 5D). STRING association network analyses revealed a large protein-protein interaction network, showing the diversity of proteins enclosed in the vesicles (Supplementary Figure 3).

Overall, these findings suggest that ectosomes may be involved in cell communication, regulating cellular metabolism and organization, and transferring immune or pathological signals between cells. 
A

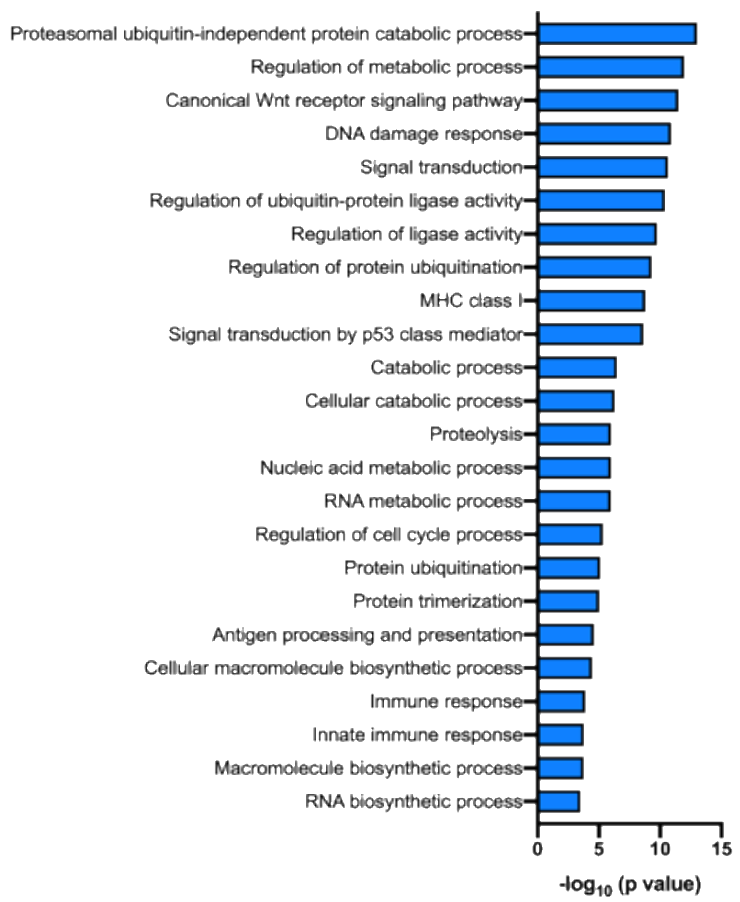

B

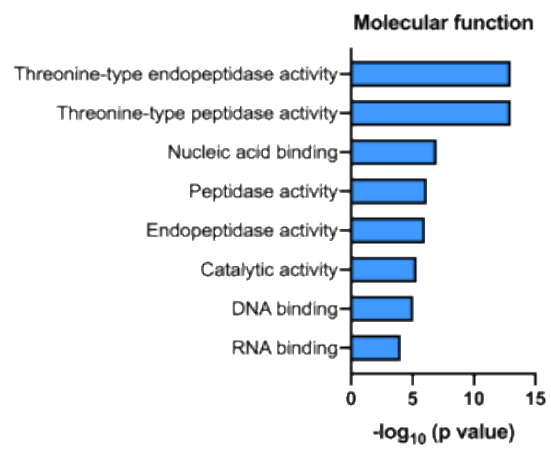

C

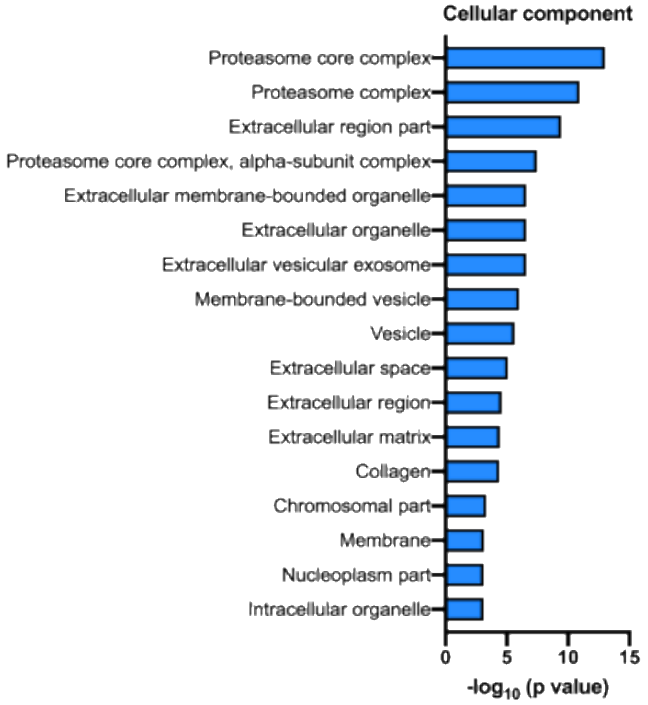

D

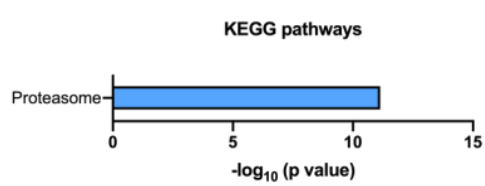

Figure 4. GO terms for proteins enriched in exosomes. The following categories were evaluated: biological process (A), molecular function (B), cellular component (C), and KEGG pathways (D). Data from five independent samples for each group was analysed using Perseus software. See also Supplementary Figure 2. 
A

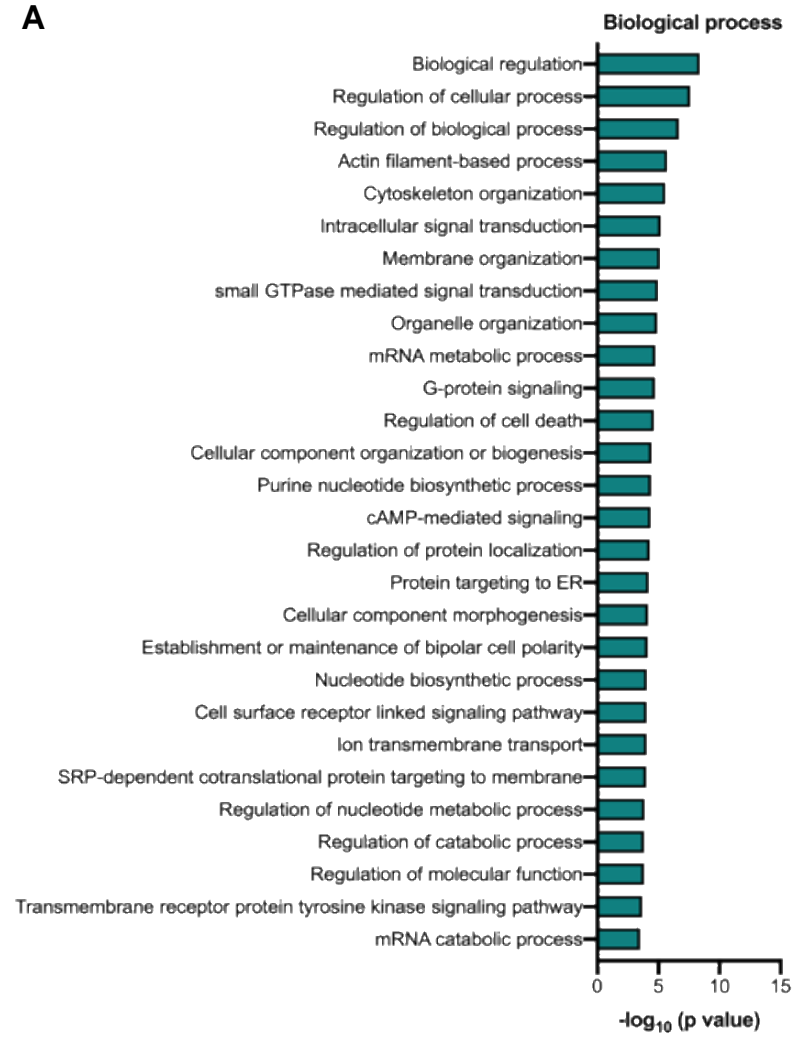

B

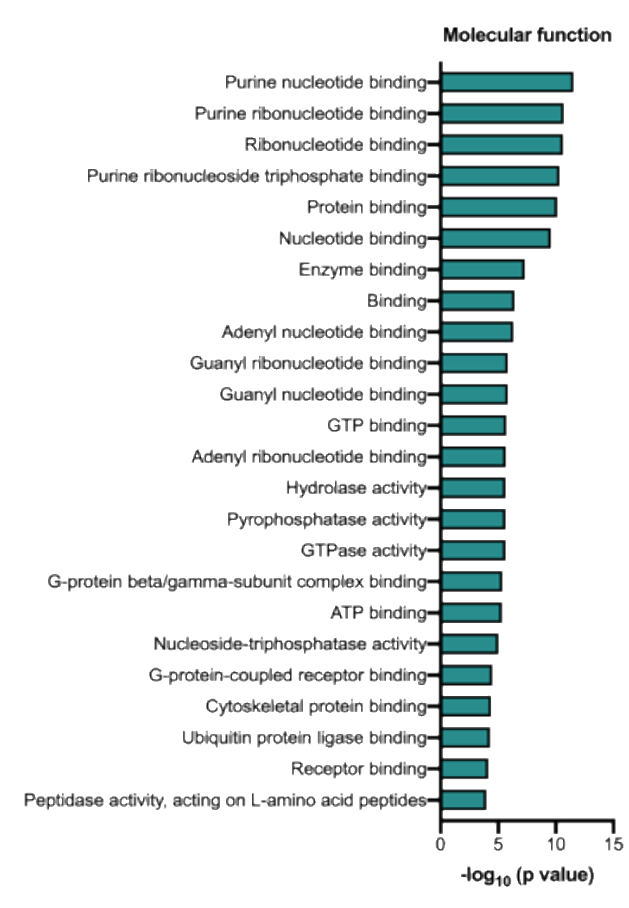

C

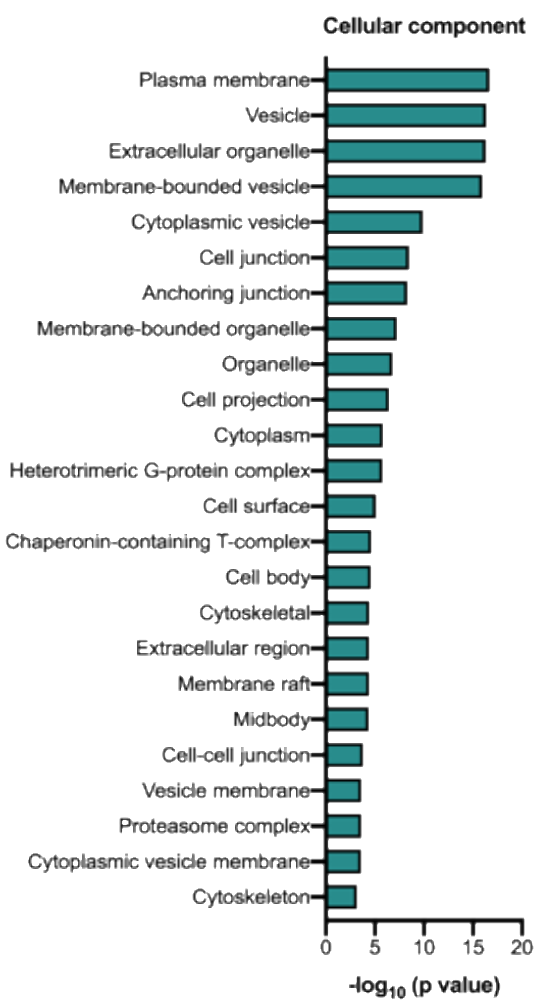

D

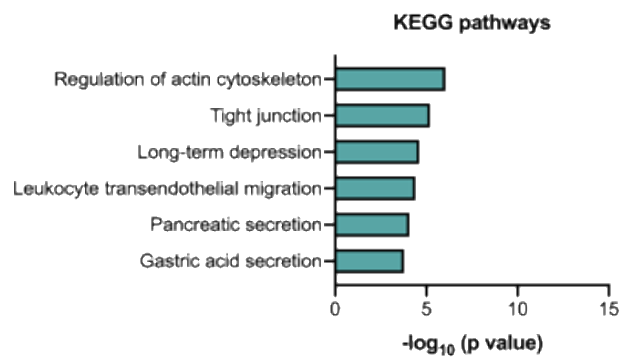

Figure 5. GO terms for proteins enriched in ectosomes. The following categories were evaluated: biological process (A), molecular function (B), cellular component (C), and KEGG pathways (D). Data from five independent samples for each group was analysed using Perseus software. See also Supplementary Figure 3. 


\section{Validation of protein markers for ectosomes and exosomes}

To confirm the protein markers identified in the proteomic datasets, ectosomes and exosomes fractions were applied into immunoblots and analysed for several of the protein hits. Staining of ectosomes and exosomes fractions showed a similar total protein profile (Figure 6A, Supplementary Figure 4A). Notably, this was markedly different from what we observed in whole the cell lysates, and we also confirmed that the conditioned media used to collect EVs did not carry residual EVs from the FBS (Supplementary Figure 4B).

In order to evaluate the EVs diversity in each fraction, the vesicles were applied into a sucrose gradient (Figure 6B). This enabled us to separate the EVs according to their floatation speed and equilibrium density, and to uncover the protein markers in each EVs type [513]. We found alix was uniformly distributed in the EVs, although in higher levels in exosomes. Interestingly, flotillin-1 was present in higher levels in light exosomes, and was slightly enriched in heavier ectosomes. Interestingly, annexin-A2 was specifically incorporated in lighter ectosomes, and was almost not detected in exosomes (Figure 6B).

Additionally, we assessed the presence of several proteins in the proteomic analyses by immunoblot and compared their distribution among EVs (Figure 6C). Consistent with previous data, alix, flotillin-1, TSG101 and CD9 were significantly enriched in exosomes (Figure 6C) [496, 497, 499]. Conversely, annexin-A2 and annexin-A5 were significantly increased in ectosomes [496] (Figure 6C). MMP2 and CD54 are commonly identified in EVs, but in our samples we did not detect significant differences in their levels in the immunoblot analyses or in the proteomic results [508, 509, 514]. Importantly, calnexin and GM130, endoplasmic reticulum and Golgi apparatus markers, respectively, were absent from our preparations, confirming no cross-contamination in the fractions due to cell death (Figure 6C) [515]. 
A

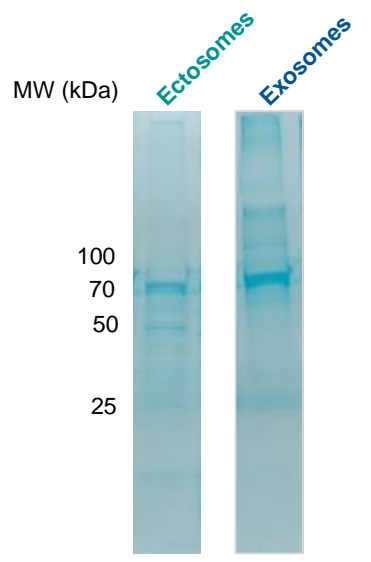

C

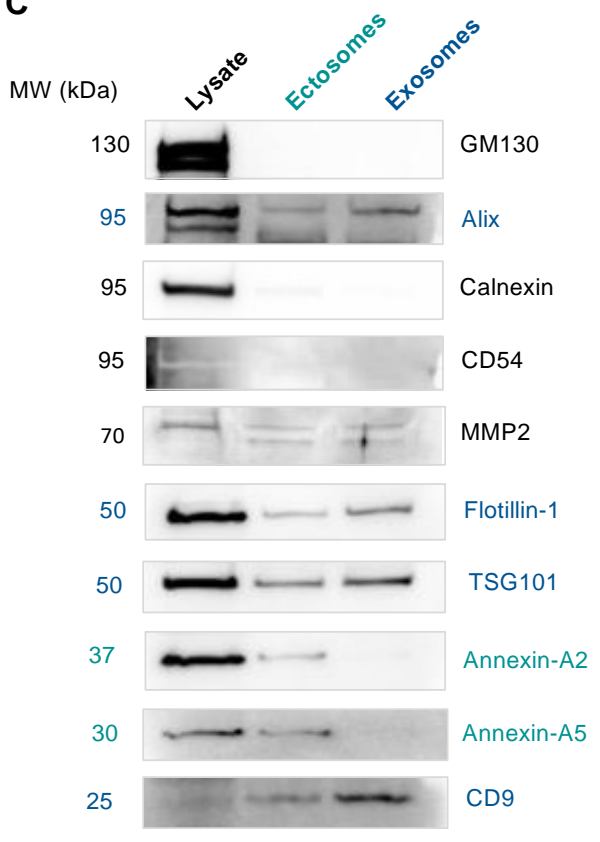

B

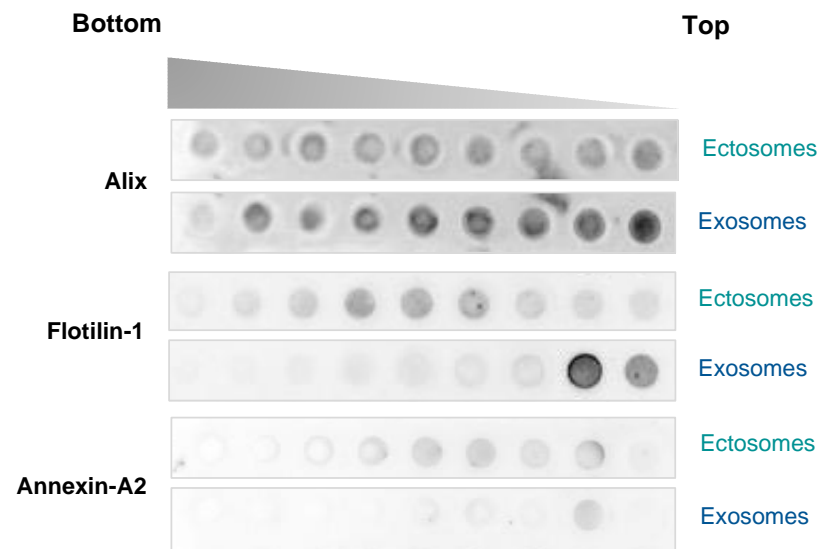

Figure 6. Validation of the proteomic profiling in ectosomes and exosomes. (A) MemCode staining demonstrates the total protein levels present in each fraction. (B) Ectosomes and exosomes were covered with a discontinuous sucrose step-gradient and fractions were applied into a Dot-Blot system and incubated with antibodies for alix, flotillin-1 and annexin-A2. (C) Immunoblots of HEK whole-cell lysates, ectosomes and exosomes fractions. Equal quantities of protein were separated on SDS-PAGE gels, and membranes were blotted with the indicated antibodies. Protein levels were normalized to total protein levels. Data from at least three independent experiments. Significant differences were assessed by one-way ANOVA followed by multiple comparisons with significance between groups corrected by Bonferroni procedure. Differences were considered to be significant for values of $p<0.05$ and are expressed as mean $\pm S D,{ }^{* \star *} p<0.001,{ }^{* \star *} p<0.0001$. See also Supplementary Figure 4 . 


\section{Markers of cell-derived EVs distinguish ectosomes and exosomes in human CSF}

CSF, the fluid bathing the central nervous system, is considered a relevant and accessible window into brain function and homeostasis. Therefore, we next asked whether the markers identified in our cell-derived EVs would enable us to distinguish EVs present in human CSF. Interestingly, ectosomes and exosomes purified from human CSF showed the presence of the same protein markers identified in EVs purified from the cell media (Figure 7). These results indicate that the protocol we used can be applied to different biofluids and confirm that the protein markers we identified are valid for EVs of different origins.

A

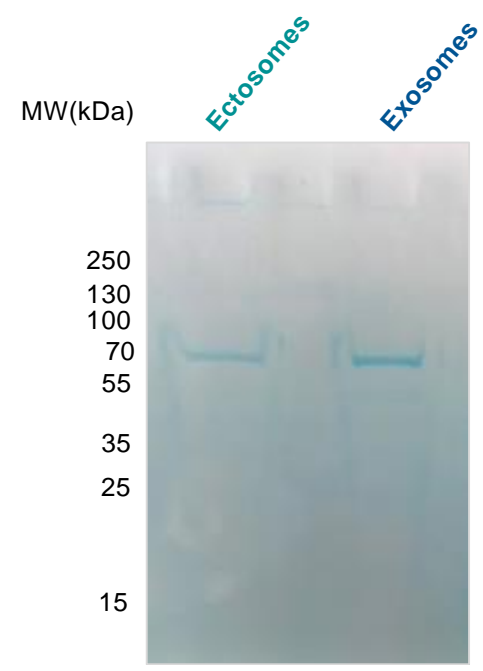

B

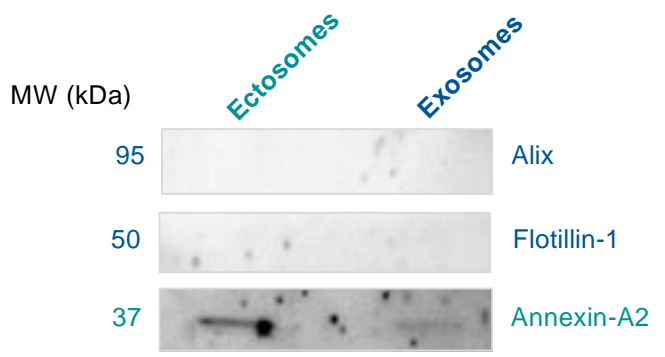

Figure 7. Presence of ectosomal and exosomal markers in human CSF. (A) Total protein levels were visualized by staining with MemCode. (B) Immunoblot validation of annexin-A2 as ectosomes marker. Alix and flotillin-1 were also evaluated in the blot.

\section{Purification and visualization of EVs using high-resolution microscopy}

Several strategies have been established to enable the visualization of EVs in internalization experiments. These include incubation with dyes that bind to the membrane, or the expression of EVs-related proteins fused to fluorescent tags [516-520]. However, these strategies present several limitations, such as the leakage of the dye to the plasma membrane after labelled-EVs are internalized, or the incomplete labelling of fluorescent vesicles due to the heterogeneity of protein markers in EVs.

Therefore, to overcome these obstacles, we developed a labelling strategy by stably expressing the green fluorescent protein (EGFP) in HEK cells and purifying ectosomes and exosomes from the cell media. As comparison, we labelled EVs using the thiol-based dye Alexa Fluor 633 C5-maleimide [521]. As expected, the EGFP signal co-localized with the 
signal of labelled-EVs (Figure 8A). Interestingly, EGFP-labelled EVs could be imaged at higher resolution when compared with dye-labelled EVs.

A
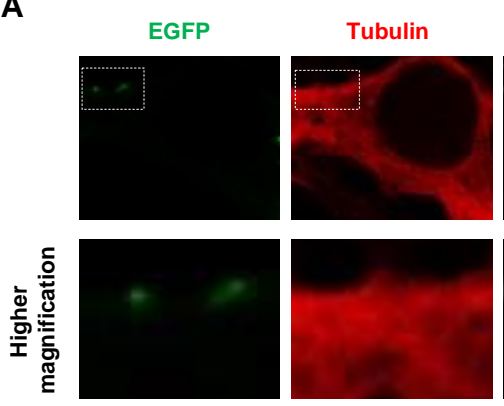

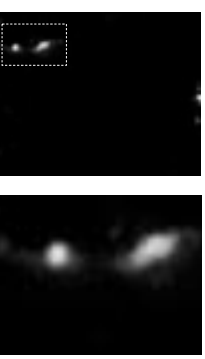

C

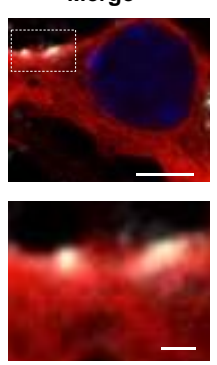

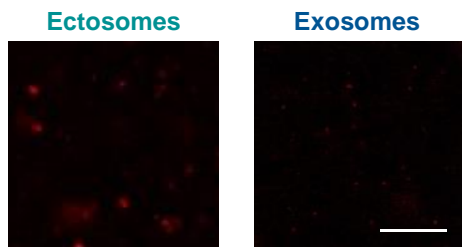

D

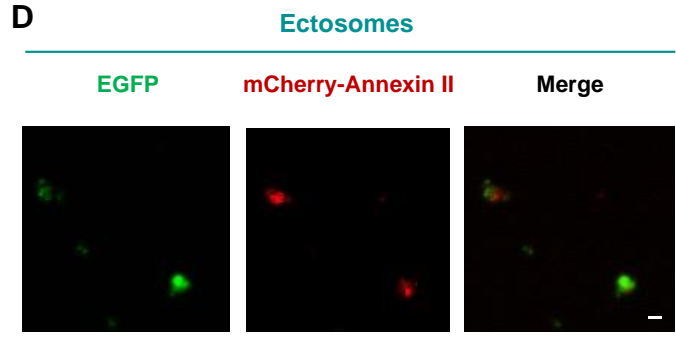

Exosomes

EGFP mCherry-Annexin II Merge
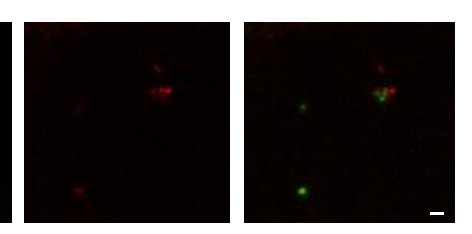

GFP
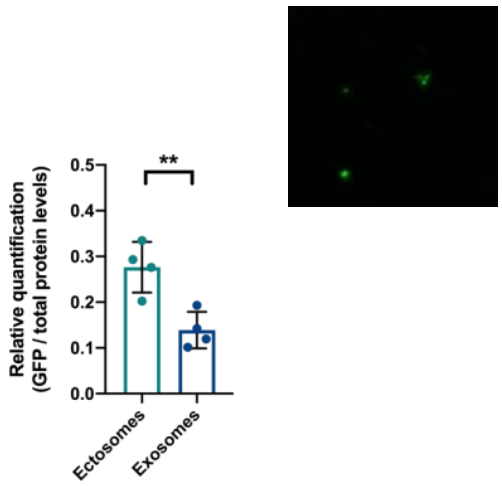

Alix

Annexin-A2

Figure 8. Visualization of ectosomes and exosomes using STED microscopy. (A) Representative image of primary cortical neurons treated with exosomes containing EGFP and labelled with thiol-based dye Alexa Fluor $633 \mathrm{C} 5$-maleimide (scale bar $5 \mu \mathrm{m}$ ). Higher magnification images of the vesicles on the cell plasma membrane (dashed boxes in white) show the colocalization signal of the double labelling strategies (scale bar $1 \mu \mathrm{m}$ ). (B) On the top, $X Y$ and $Z X$ axis STED imaging of purified ectosomes and exosomes from HEK cells expressing EGFP tag (scale bars represent $1 \mu \mathrm{m}$ in $X Y$ and $Z X$ axis). On the bottom, immunoblot of the vesicles displaying the incorporated levels of EGFP and respective vesicle protein markers. The immunoblot lanes represent a montage from the same blot. (C) STED imaging of purified ectosomes and exosomes from HEK cells expressing mCherryAnnexin-A2 (scale bar $5 \mu \mathrm{m}$ ). (D) STED images of purified ectosomes and exosomes containing EGFP and mCherry-Annexin-A2 (scale bar $1 \mu \mathrm{m}$ ). See also Supplementary Figure 5. 
When the EGFP-positive vesicles were visualized by stimulated emission depletion (STED) microscopy, ectosomes exhibited stronger fluorescence than exosomes (Figure 8B). Additionally, ectosomes incorporated higher levels of EGFP compared with exosomes, independent of their size or total protein levels (quantifications were normalized to total protein levels) (Figure 8B, Supplementary Figure 5A). The higher incorporation of EGFP in ectosomes might be related with its cytosolic expression, making it more available for incorporation into ectosomes. This is consistent with our finding of a greater number of cytoplasmatic proteins in ectosomes than in exosomes.

Next, we further evaluated this labelling strategy and constructed an mCherry-annexinA2 stable cell line. Ectosomes showed stronger fluorescence intensity for annexin-A2 when compared with exosomes, further validating the enrichment of annexin-A2 in these vesicles (Figure 8C, Supplementary Figure 5B). Additionally, ectosomes purified from cells coexpressing EGFP and mCherry-annexin-A2 exhibited the co-localization of both signals (Figure 8D). mCherry-annexin-A2 was also detected in exosomes using STED microscopy, albeit at lower levels (Figure 8D, Supplementary Figure 5B).

In summary, stable expression of EGFP in cells allows the purification of fluorescently labelled EVs from media of cultured cells for high-resolution imaging studies without changing the levels of specific protein markers.

\section{Annexin-A2 as specific marker for ectosomes}

Annexins are abundant membrane-associated proteins that have been identified as EVs constituents [295, 496, 499, 500]. In our proteomic analyses, we found several annexin proteins enriched in ectosomes, such as annexin-A2 (Figure 3F, Supplementary Table 2). Using sucrose gradients and immunoblot analyses, we observed that annexin-A2 is markedly present in ectosomes (Figure 6B and C). Expression of mCherry-annexin-A2 in cells showed its higher incorporation in ectosomes using STED microscopy (Figure 8C-D, Supplementary Figure 5). Remarkably, ectosomes purified from human CSF also demonstrated an enrichment in annexin-A2 (Figure 7). Together, these results indicate that annexin-A2 is a specific marker of ectosomes from different origins.

\section{Neuronal cells take up ectosomes and exosomes at similar levels}

The uptake of EVs by recipient cells is a key step for intercellular communication. Therefore, we next investigated the internalization and intercellular transfer of both ectosomes and exosomes in vitro. Primary cortical neurons were seeded in microfluidic devices in order 
to separate neuronal cell bodies from their axons and from second-order neurons that would be contacted by those axons (Figure 9A, Supplementary Figure $6 \mathrm{~A}$ and $\mathrm{B}$ ).

Fluidic isolation was achieved using a volume difference between the soma and axon chambers [522]. At DIV14, neurons were treated with an equal amount of exosomes or ectosomes enriched with EGFP $(20 \mu \mathrm{g} / \mathrm{mL})$ in the upper left well (input) (Figure 9A, Supplementary Figure $6 \mathrm{~A}$ and $\mathrm{B}$ ). Both ectosomes and exosomes were taken up and transferred between neurons. Visualization of the axons in the microgrooves revealed the colocalization of EGFP from the EVs with tubulin staining, demonstrating their internalization and transport in the cells (Figure 9A).

To determine the internalization levels of ectosomes and exosomes in primary cortical neurons, Alexa Fluor 633 C5-maleimide-labelled EVs, with and without EGFP, were added to the medium at DIV14 to a final concentration of $20 \mu \mathrm{g} / \mathrm{mL}$. After 24 hours, cells were fixed, immunostained for MAP2 and analysed by confocal microscopy. Labelled EVs were observed as puncta predominantly in the cytoplasm of cells, confirming the internalization of ectosomes and exosomes (Figure 9B). PBS-treated neurons did not show positive signal for the dye, indicating that the dye efficiently bound to EVs (Figure 9B). Interestingly, ectosomes and exosomes were internalized at similar levels and the signal of EGFP and dye co-localized for double-labelled EVs (Figure 9B). Additionally, EVs treatment was not toxic to the neurons (Supplementary Figure 6C). Interestingly, after internalization, the EVs-EGFP signal was surrounded by LC3 staining, suggesting a possible engulfment and degradation of EVs in primary cortical neurons (Figure 9C). 
A
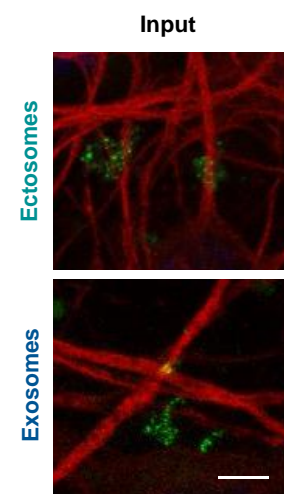

B
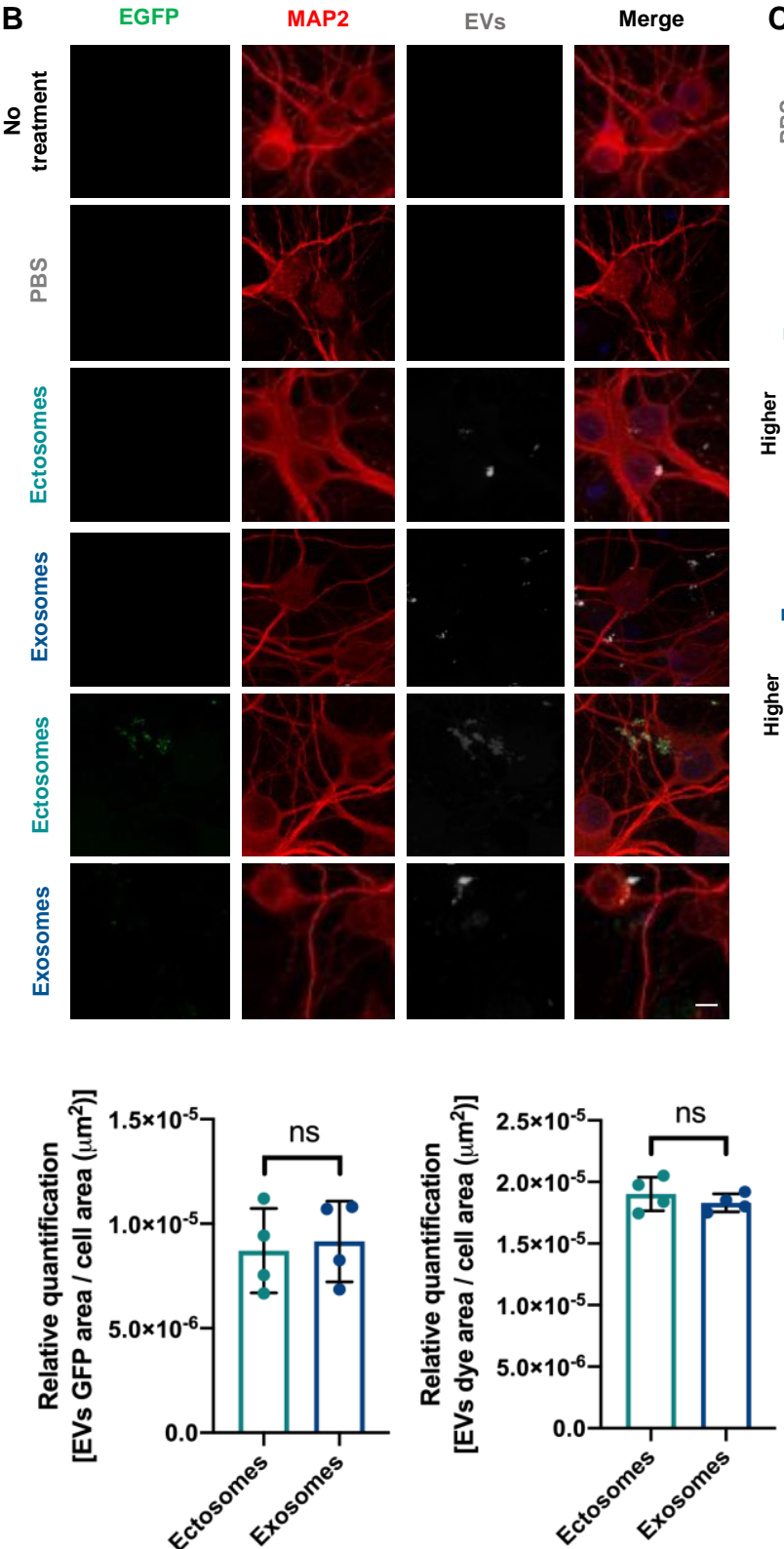

Figure 9. Internalization and axonal transport of EVs by primary cortical neurons. (A) Primary cortical neurons were seeded in microfluidic devices to separate neuronal cell bodies. On the left, EVs containing EGFP were added to primary cortical neurons at DIV14 for 24 hours in the upper left input well $(20 \mu \mathrm{g} / \mathrm{mL})$. On the right, 
representative images of cortical neurons immunostained for tubulin demonstrating cytoplasmic internalization and axonal transport of ectosomes and exosomes. White arrowheads show the fluorescent signals within the cell (scale bar $5 \mu \mathrm{m}$ ). (B) Primary cortical neurons were treated with $20 \mu \mathrm{g} / \mathrm{mL}$ EVs at DIV14 for 24 hours. Ectosomes and exosomes without and with EGFP were previously labelled with the fluorescent dye Alexa Fluor 633 C5-maleimide. As a control, dye was incubated with PBS and monitored in the same manner. Representative images of cortical neurons immunostained for MAP2 to demonstrate cytoplasmic internalization of different EVs subtypes (scale bar $5 \mu \mathrm{m})$. Quantification of the internalized vesicles was performed by measuring the EVs dye or EGFP signal area per cell area internalized by neuronal cells. Data from at least three independent experiments. Significant differences were assessed by two-tailed unpaired t-test comparison and are expressed as mean \pm SD. (C) Primary cortical neurons were treated with $20 \mu \mathrm{g} / \mathrm{mL}$ of EGFP-enriched EVs at DIV14 for 24 hours. Representative images of cortical neurons immunostained for LC3 and cell membrane was labelled using WGA dye. White arrowhead indicates the fluorescent signals within the cell and higher magnification images are presented below each panel (scale bar $5 \mu \mathrm{m}$ ). See also Supplementary Figure 6.

\section{Ectosomes modulate spontaneous activity of cortical neuronal networks}

Finally, we assessed the functional relevance of EVs. Since several cell types in the brain actively release $\mathrm{EVs}$ to the extracellular space, we hypothesized that neuronal function might be modulated by ectosomes and/or exosomes [523-526]. Therefore, we used multielectrode arrays (MEAs) to evaluate the effect of EVs internalization on spontaneous activity in primary cortical neuronal cultures [527]. Cells were cultured in MEA chambers until DIV14 to allow the establishment of mature neuronal networks, and spontaneous firing activity was recorded 24 hours after incubation with $20 \mu \mathrm{g} / \mathrm{mL}$ of EVs (Figure 10, Supplementary Figure 7). Representative raster plots and voltage traces show the typical firing activity and bursts events in neuronal cultures treated with PBS, ectosomes or exosomes (Figure 10A). Interestingly, assessment of bursting activity parameters showed that under EVs treatment, spikes bursts came more irregularly and showed increased duration (Figure 10B). Exosomes internalization also resulted in longer inter-burst intervals, decreased intra-burst spiking frequency, and a smaller percentage of spikes within the bursts, in contrast to ectosomes (Figure 10B). Although not significant, EVs treatment caused a slight reduction in the burst rate (Supplementary Figure 7C). In our cultures, we also observed a reduction in the mean firing rate after EVs internalization, with decrease of the average spike amplitude for the neurons treated with ectosomes (Supplementary Figure 7D). 
A
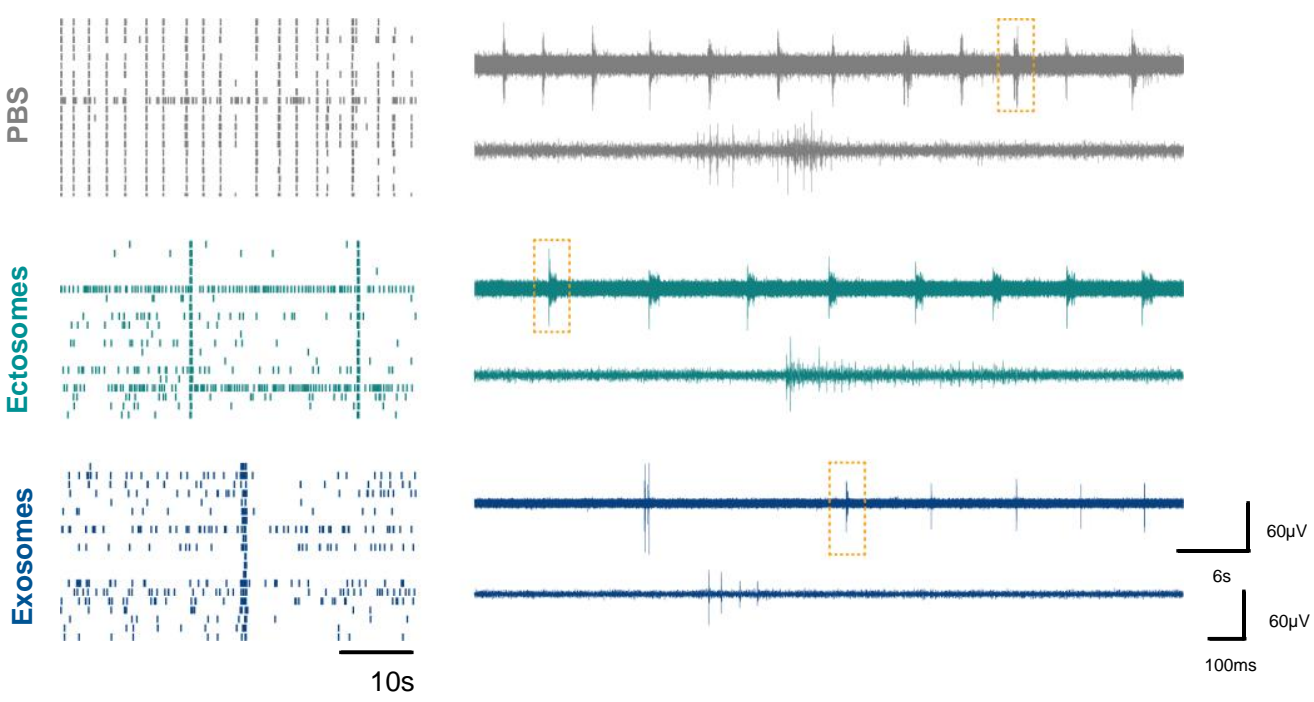

B
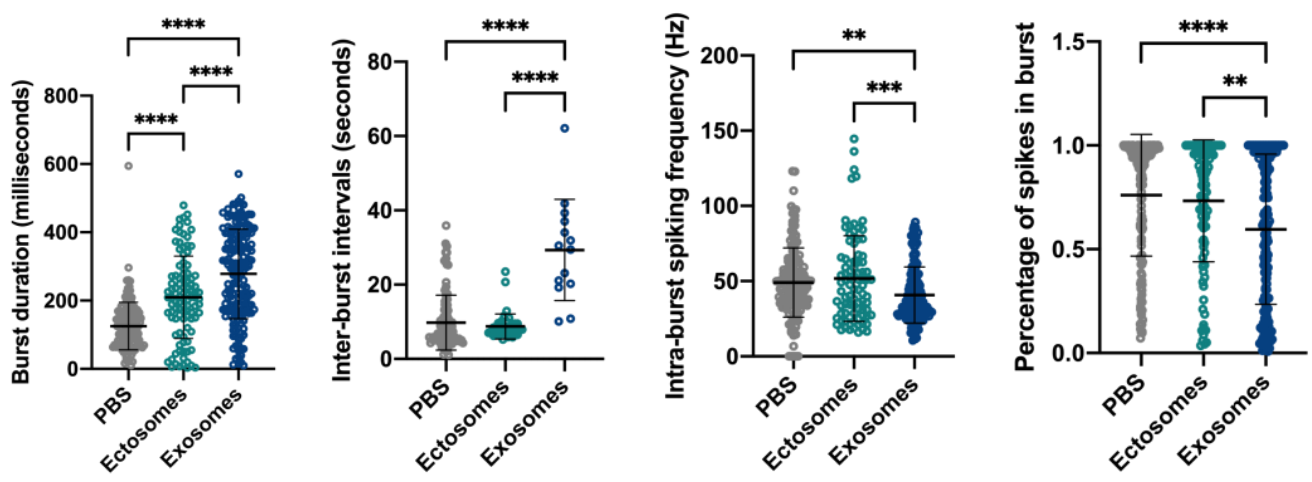

Figure 10. EVs modulate spontaneous activity in primary cortical neurons. (A) On the left, representative raster plots of the spontaneous firing activities recorded from cortical neurons after incubation with $20 \mu \mathrm{g} / \mathrm{mL}$ of $E V s$ for 24 hours at DIV14, recorded using 60-electrode MEAs. Each row represents one single cell (20 cells shown) and each vertical line represents a single spike obtained on DIV15 [scale bar represents 10 seconds (s)]. On the right, representative voltage traces showing the typical firing activity and bursts events in neuronal cultures treated with PBS, ectosomes or exosomes (upper traces, scale bars represent $60 \mu \mathrm{V}$ and $6 \mathrm{~s}$ ). Closeups of the dashed boxes represent the spikes occurring within a burst ([lower traces, scale bars represent $60 \mu \mathrm{V}$ and 100 milliseconds (ms)]. (B) Bursting properties of the cortical neurons treated with EVs (burst duration, inter-burst intervals, intraburst spiking frequency and percentage of spikes in bursts). Data from at least three independent experiments for each condition. Significant differences were assessed by one-way ANOVA followed by multiple comparisons with significance between groups corrected by Bonferroni procedure. Differences were considered to be significant for values of $p<0.05$ and are expressed as mean $\pm S D,{ }^{* *} p<0.01,{ }^{* * *} p<0.001^{* * * *}, p<0.0001$. See also Supplementary Figure 7.

Altogether, these results suggest that ectosomes and exosomes can modulate important parameters of neuronal spontaneous activity and make the cultured neurons fire in a less synchronized and more irregular fashion. 


\section{Discussion}

EVs are important vehicles for remote intercellular communication and signalling. These vesicles encapsulate proteins, RNAs, lipids and signalling molecules that reproduce the cellular content of the origin cell and can modulate several processes in the recipient cells [528-530]. It is unclear how many different types of EVs are secreted by each cell type. The diversity in their size and content suggests that cells may secrete a large number of different types of vesicles, reflecting distinct physiological roles [310].

The use of EVs as biomarkers has attracted significant interest in several areas of study. However, the use of different EVs purification methods, the absence of reliable markers, and the lack of comprehensive characterization, caused an accumulation of contradictory data and challenges in the study of EVs biology [295, 499, 500, 531]. Numerous studies have focused on the characterization and study of the biological function of exosomes. However, ectosomes remain largely understudied [528, 530, 532, 533]. Thus, understanding the role of ectosomes in intercellular communication, the mechanisms involved in their biogenesis, and the characterization of their content will shed light into their biological function and into how they may be used as disease biomarkers.

In our study, we developed a differential ultracentrifugation protocol to efficiently and reproducibly isolate ectosomes and exosomes from diverse biofluids, including human CSF. We observed that ectosomes are larger and are released from cells in higher quantities when compared to exosomes.

Inclusion or exclusion of cellular proteins into EVs appears to be based on controlled protein-sorting mechanisms during their biogenesis, rather than simply on protein abundance in the cell, in agreement with unique protein signatures for each EV subtype. For example, it is known that exosomes are distributed in subpopulations displaying distinct compositions and/or functions [306, 499]. Slight differences in protein content are also expected depending on the isolation protocol and cell type/tissue of origin. Our comprehensive proteomic analyses revealed singular proteomic profiles for ectosomes and exosomes that enabled us to establish protein markers that can be used for their biochemical distinction [309, 496, 499]. We have identified several membrane-associated proteins, such as annexin-A2 and annexin-A5, as markers for ectosomes. Furthermore, we confirmed that human CSF-derived ectosomes are also enriched in annexin-A2. As previously shown, CD9 and alix are specific markers for cell and CSF-derived exosomes [299, 496, 499].

EVs in any biofluid comprise diverse subpopulations that can differ in composition and biogenic mechanisms. Therefore, the variety of machineries involved in the generation of EVs will result in differences in protein composition, lipids and RNA, thereby determining their 
function and fate. In our study, we demonstrate that ectosomes and exosomes reflect distinct biological processes in the cells. The assessment of ectosomal content highlighted proteins and enzymes involved in processes occurring in the cytosol and plasma membrane. In contrast, exosomal content revealed proteins involved in precise biological processes, such as proteasomal activity. These results indicate that evaluation of different EVs from the same biofluid will provide comprehensive information on biological processes and on the status of the cells. Also, their composition might differ under normal and stress conditions, informing on the signals and cargoes transferred between cells, and reflecting physiological and pathological statuses.

Fluorescent labelling of EVs to investigate their internalization in cells remains challenging due to possible changes in their functionality. The majority of labelling strategies include the use of dyes which bind non-covalently to the membrane bilayer. However, dyes can aggregate or transfer signal to the cell plasma membrane after treatment with labelledEVs, resulting in misleading results from uptake experiments [516-518]. Other labelling methods include the use of stable cell lines that fuse GFP to protein markers in EVs [519, 520]. Subsequently, only a subpopulation of EVs will be fluorescent due to EVs heterogeneity. Therefore, we fluorescently labelled EVs through the stable expression of EGFP in cells. EGFP signal co-localized with the signal of labelled-EVs using a thiol-based dye [521]. Interestingly, the EGFP signal allowed the imaging of EVs internalization with high-resolution, which will be useful for future studies of EVs biology. Furthermore, EGFP is more incorporated in ectosomes than in exosomes, likely as a result of EGFP availability in the cytoplasm and due to the distinct origins of the EVs. We also observed that stable expression of mCherryannexin-A2 resulted in its incorporation in higher levels in ectosomes than in exosomes, further confirming the use of annexin-A2 as an ectosomal marker. In summary, in our study we developed a simple and practical tool to fluorescently label EVs derived from cultured cells. This strategy takes advantage of the cytosolic expression of EGFP, without compromising the protein composition of the EVs.

After the detailed biochemical characterization of both ectosomes and exosomes, we evaluated their internalization and functional implications in neuronal activity by treating primary cortical neurons with the different EVs. Neurons take up and transport both ectosomes and exosomes between cells and do not display any detectable signs of neurotoxic effects. The LC3 staining surrounding the EVs after internalization suggests at least a fraction enters in the cells as intact vesicles and are sorted for degradation by autophagy.

Ectosomes and exosomes carry a complex assortment of biomolecules with distinct activities that become internalized by receptor cells, most probably producing distinct functional outputs. EVs have been hypothesized to play important roles in the nervous system 
[295]. However, the potential role of ectosomes and exosomes in neural function is unclear. Assessment of neuronal network activity using MEA recordings demonstrated that spontaneous neuronal activity can be modulated by ectosomes and exosomes. Most importantly, EVs treatment disrupted the regular synchronized bursting activity neuronal cultures, resulting in overall lower and more disorganized spiking activity. We hypothesize that this reorganization of concerted neuronal activity might result from EVs acting on the presynaptic site, where they may decrease the release of synaptic vesicles. Previous studies showed that exosomes purified from microglial and oligodendroglial cells can also modulate neuronal activity $[523,526]$. However, the impact of EVs on neuronal excitability in vivo remains understudied.

Altogether, our study forms the foundation for future studies of EVs biology, demonstrates distinct biogenesis and, importantly, confirms distinct functional effects of exosomes and ectosomes in neuronal networks in vitro. Moreover, our work will aid efforts to discover future biomarkers for different human pathological conditions.

\section{Acknowledgments}

We thank Sabine König and Uwe Plessmann from Max Planck Institute for Biophysical Chemistry (Göttingen) and Christof Lenz from the Core Facility Proteomics at University Medical Center Göttingen (Göttingen) for helping with mass spectrometry analysis. TFO is supported by European Union's Horizon 2020 research and innovation program under grant agreement No. 721802 (SynDegen), and by the Deutsche Forschungsgemeinschaft (DFG, German Research Foundation) under Germany's Excellence Strategy - EXC 2067/1390729940, by SFB1286 (B8). TG is supported by the European Research Council (ERC) under the European Union's Horizon 2020 research and innovation programme (grant agreement number 724822). This work was partly supported by the Göttingen Graduate School for Neurosciences, Biophysics, and Molecular Biosciences (DFG grant GSC 226/4). Annexin-A2-GFP plasmid was kindly provided by Volker Gerke \& Ursula Rescher (Plasmid \#107196, Addgene) (Rescher, Zobiack et al. 2000). Figures were created with BioRender.com.

\section{Author contributions}

T.F.O. and I.C.B. conceived the study. I.C.B performed all the cell culture, molecular biology and imaging experiments. M.H.K and I.C.B stablished the spike sorting framework, performed MEA experiments and data analysis. D.R performed the EM experiments. I.P. evaluated the mass spectrometry data using MaxQuant. E.G prepared the lentiviral vectors 
used in the study. C.V.R provided the CSF material for the study. T.G. provided methodology and resources for the MEA experiments. I.C.B analysed and interpreted the data. I.C.B generated the graphs and figures. I.C.B. and T.F.O. wrote the manuscript. T.F.O. supervised the work.

\section{Declaration of Interests}

The authors declare no competing interests.

\section{Materials and Methods}

\section{Resource availability}

\section{Lead contact for reagent and resource sharing}

Further information and requests for resources and reagents should be directed to and will be fulfilled by the Lead Contact, Tiago F. Outeiro (touteir@gwdg.de).

\section{Data and code availability}

Original data generated by this study are available upon request.

\section{Experimental model and subject details}

\section{Human cerebrospinal fluid samples}

Human cerebrospinal fluid (CSF) samples were obtained with informed consent from adult volunteers and all experiments were performed in accordance with relevant guidelines and regulations. Samples were obtained from the CSF bank of the University Medical Center Göttingen (Göttingen, Germany). Samples were classified according to the disease diagnostics and information about sex and age were anonymized. CSF samples were stored in $0.5,1.0$ and $1.5 \mathrm{~mL}$ aliquots at $-80^{\circ} \mathrm{C}$ prior to analysis and were pooled for extracellular vesicles (EVs) isolation (10mL final volume).

\section{Neuronal culture}

Primary cortical neuronal cultures were prepared from C57BL6/J\#00245 wild-type E15.5 embryos from the animal facility of the University Medical Center Göttingen (Göttingen, Germany), as previously described [534]. C57BL6/J\#00245 background mice were bred and maintained under specific pathogen free conditions in the animal facility of the University Medical Center Göttingen (Göttingen, Germany). Animal procedures were performed in accordance with the European Community (Directive 2010/63/EU), institutional and national 
guidelines (license number 19.3213). In detail, pregnant animals were sacrificed by carbon dioxide intoxication and the embryos extracted from the uterus. The meninges were removed, and the cortex was dissected under a stereomicroscope, and afterwards the tissue was transferred to ice-cold $1 \times$ Hanks balanced salt solution ( $\mathrm{CaCl}_{2}$ and $\mathrm{MgCl}_{2}$ free; HBSS) (Gibco Invitrogen, CA, USA) supplemented with $0.5 \%$ sodium bicarbonate solution (Sigma-Aldrich, MO, USA). After trypsinization at $37^{\circ} \mathrm{C}$ for 15 minutes (min) $(100 \mu \mathrm{L}$ of $0.25 \%$ trypsin; Gibco Invitrogen, CA, USA), the reaction was stopped by adding $100 \mu \mathrm{L}$ DNase I $(0.5 \mathrm{mg} / \mathrm{mL}$, Roche, Basel, Switzerland) and 100 $\mathrm{LL}$ fetal bovine serum (FBS) (Anprotec, Bruckberg, Germany). The tissue was gently dissociated using a glass pipette. After centrifugation at $300 \times \mathrm{x}$ for 5 min, cells were resuspended in pre-warmed neurobasal medium (Gibco Invitrogen, CA, USA) supplemented with 2\% B27 supplement (Gibco Invitrogen, CA, USA), 1\% penicillinstreptomycin (PAN Biotech, Aidenbach, Germany) and 0.25\% GlutaMAX (Gibco Invitrogen, CA, USA). Cells were seeded on coverslips coated with poly-L-ornithine $(0.1 \mathrm{mg} / \mathrm{mL}$ in borate buffer; PLO) (Sigma-Aldrich, MO, USA) or culture plates (Corning, Merck, Darmstadt, Germany) for immunocytochemistry and western blot experiments. Cells were maintained at $37^{\circ} \mathrm{C}$ with $5 \% \mathrm{CO}^{2}$, and fresh medium was added every 3-4 days.

\section{Human embryonic kidney cells}

Human embryonic kidney (HEK) 293 cells (ATTC, VA, USA) were maintained in DMEM medium (PAN Biotech, Aidenbach, Germany) supplemented with 10\% FBS (Anprotec, Bruckberg, Germany) and 1\% penicillin- streptomycin (PAN Biotech, Aidenbach, Germany) at $37^{\circ} \mathrm{C}$ in a $5 \% \mathrm{CO}^{2}$ atmosphere. Stable cell lines expressing pRRL-CMV-EGFP-PRE-SIN, FUGW-mCherry-Annexin-A2 or FUGW-mCherry were developed by lentiviral infection of HEK 293 cells. Cells were incubated during 5 days with the virus and after three passages the infection rate was confirmed by microscopy (more than $90 \%$ of positive cells).

\section{Method details}

\section{Lentivirus production protocol}

Production of lentivirus pRRL-CMV-EGFP-PRE-SIN, FUGW-mCherry-Annexin-A2 and FUGW-mCherry was performed as previously described [535]. HEK 293 cells were seeded in culture plates (Corning, Merck, Darmstadt, Germany) and incubated overnight at $37^{\circ} \mathrm{C}$ with $5 \% \mathrm{CO}^{2}$ in DMEM (PAN Biotech, Aidenbach, Germany) supplemented with 10\% FBS (Anprotec, Bruckberg, Germany) and 1\% penicillin-streptomycin (PAN Biotech, Aidenbach, Germany). On the next day, cells were incubated with transfection medium containing DMEM with 2\% FBS (Anprotec, Bruckberg, Germany) for 5 hours (h) before 
transfection. Calcium phosphate $\left(\mathrm{CaPO}_{4}\right)$ precipitation method was used for transfection with

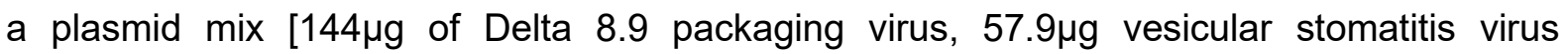
glycoprotein (VSVG) packing virus and 160 $\mu$ g of the plasmid of interest]. DNA mix was added to $6 \mathrm{~mL}$ of $1 \times$ BBS (50 mM BES, $280 \mathrm{mM} \mathrm{NaCl}, 1.5 \mathrm{mM} \mathrm{Na}_{2} \mathrm{HPO}_{4}$ ) and $0.36 \mathrm{~mL} \mathrm{CaCl}_{2}$ (2.5M $\mathrm{CaCl}_{2}$ ) was added to the mixture in a vortex shaker. Solution was incubated $20 \mathrm{~min}$ in the dark before adding to the cells. On the next day, the medium was changed to Panserin (PAN Biotech, Aidenbach, Germany) supplemented with $1 \%$ of non-essential amino acids (MEM, Gibco Invitrogen, CA, USA) and 1\% penicillin- streptomycin (PAN Biotech, Aidenbach, Germany). Viruses were harvested $72 \mathrm{~h}$ after transfection, centrifuged at $3000 \mathrm{xg}$ for $15 \mathrm{~min}$ at $4^{\circ} \mathrm{C}$. The supernatant was cleared of cell debris by filtering through a $0.45 \mu \mathrm{m}$ filter (Corning, Merck, Darmstadt, Germany) and mixed with 1x PEG solution (SBI System Bioscience, CA, USA) to pellet the virus. After 2 days of incubation at $4^{\circ} \mathrm{C}$, viruses were spin down by centrifugation at $1500 \mathrm{xg}$ during $30 \mathrm{~min}$ at $4{ }^{\circ} \mathrm{C}$. Supernatant was discarded and the pellet was resuspended in Panserin (PAN Biotech, Aidenbach, Germany).

\section{Microfluidic chambers}

Triple compartment neuron silicone devices (TCND1000) with 2 microgroove barriers of $500 \mu \mathrm{m}$ with a $1000 \mu \mathrm{m}$ central compartment were obtained from Xona microfluidics and prepared for neuronal cell culture as previously described $[522,536]$ and following the manufacturer's instructions (Xona microfluidics, NC, USA). Experiments were performed as previously described [537]. Cover glass $24 \mathrm{~mm}$ x 40mm (Corning, Merck, Darmstadt, Germany) and microfluidic devices were rinsed with 70\% ethanol (Sigma-Aldrich, MO, USA) and water under sterile conditions. Cover glass were coated with poly-L-lysine $(0.5 \mathrm{mg} / \mathrm{mL}$ in borate buffer; PLL) (Sigma-Aldrich, MO, USA). After bonding the microfluidic devices to glass coverslips, neurons were seeded in the soma channels and incubated at $37^{\circ} \mathrm{C}, 5 \% \mathrm{CO}^{2}$, in a humidified incubator. Furthermore, the second and third chambers were filled with more neuronal cell media than in the first chambers. The volume difference between the chambers resulted in continuous hydrostatic pressure barrier, which also prevented diffusion of EVs from the treated chamber into others. Treatment was performed at days in vitro (DIV) 14 and the cells fixed for immunocytochemistry at DIV15 (see immunocytochemistry section).

\section{Immunocytochemistry experiments}

After treatment, primary and cell lines cultures were first washed with 1x PBS (PAN Biotech, Aidenbach, Germany) and fixed with $4 \%$ of paraformaldehyde solution (PFA) for $20 \mathrm{~min}$ at room temperature (RT). In order to quench PFA autofluorescence, samples were incubated with $50 \mathrm{mM}$ of ammonium chloride $\left(\mathrm{NH}_{4} \mathrm{Cl}\right)$ solution for $30 \mathrm{~min}$. In order to label the 
plasma membrane, cells were incubated during 10 min with wheat germ agglutinin (WGA) Alexa FluorTM 633 conjugate in 1x HBSS at RT (5.0 $\mathrm{gg} / \mathrm{mL}$; W21404, Invitrogen, CA, USA). Cells were washed with 1x PBS (PAN Biotech, Aidenbach, Germany). Cells were permeabilized with $0.1 \%$ Triton X-100 (Sigma-Aldrich, MO, USA) for 10min. Following permeabilization, cells were blocked with $2 \%$ bovine serum albumin (BSA) in 1x PBS (SigmaAldrich, MO, USA) for $1 \mathrm{~h}$ at RT and then incubated with the primary antibodies overnight at $4^{\circ} \mathrm{C}$ [alpha-tubulin (1:500, T9026, Sigma-Aldrich), MAP2 (1:500, 17490-1-AP, Proteintech), LC3 (1:500, PM036, MBL International)]. Afterwards, the cells were washed with 1x PBS (PAN Biotech, Aidenbach, Germany) and then with fluorescence conjugated secondary antibodies for $2 \mathrm{~h}$ at RT [Alexa Fluor 555 donkey anti-rabbit (1:1000, A31572, Invitrogen), Alexa Fluor 555 donkey anti-mouse (1:1000, A31570, Invitrogen)]. Finally, nuclei were counter-stained with DAPI (Carl Roth, Karlsruhe, Germany) and mounted with mowiol for microscopy.

\section{Western blots}

Cultured cells were collected and lysed in RIPA buffer (50 mM Tris, pH 8.0, 0.15 M $\mathrm{NaCl}, 0.1 \%$ SDS, $1.0 \%$ NP-40, 0.5\% Na-Deoxycholate, 2mM EDTA, supplemented with protease and phosphatase inhibitors cocktail (cOmplete ${ }^{\mathrm{TM}}$ protease inhibitor and PhosSTOPTM phosphatase inhibitor; Roche, Basel, Switzerland). Protein concentrations in the lysates were determined by the Bradford protein assay (Bio-Rad, CA, USA). $30 \mu \mathrm{g}$ of protein were denaturated $5 \mathrm{~min}$ at $95^{\circ} \mathrm{C}$, loaded into $12 \%$ SDS-PAGE gels and transferred to nitrocellulose membranes using iBlot2 (Invitrogen, CA, USA). Membranes were blocked with 5\% skim milk (Sigma-Aldrich, MO, USA) in Tris-buffered saline (pH 8) with $0.05 \%$ Tween 20 (TBS-T) and then incubated with the appropriate primary antibody overnight in 5\% BSA (Sigma-Aldrich, MO, USA) in TBS-T at $4^{\circ} \mathrm{C}$ [alix (1:1000, ab117600, Abcam), annexin-A5 (1:1000, 8555, Cell signaling), annexin-A2 (1:1000, ab178677, Abcam), calnexin (1:1000, ab22595, Abcam), CD54 (1:1000, 4915, Cell signaling), CD9 (1:1000, 13174, Cell signaling), flotilin-1 (1:1000, 18634, Cell signaling), GFP (1:1000, 11814460001, Roche), GM130 (1:1000, 12480, Cell signaling), MMP2 (1:1000, sc-13595, Santa Cruz), TSG101 (1:1000, ab30871, Abcam), mCherry (1:1000, ab167453, Abcam)]. After three washes with TBS-T, membranes were incubated for $2 \mathrm{~h}$ with horseradish peroxidase (HRP) conjugated secondary antibodies [ECL ${ }^{\mathrm{TM}}$ Mouse IgG (1:10000, NXA931, Amersham), ECL ${ }^{\text {TM }}$ Rabbit IgG (1:10000, NA934V, Amersham)]. After incubation with the secondary antibody, membranes were washed three times with TBS-T and developed in a chemiluminescence system (Fusion FX Vilber Lourmat, Vilber, France) using chemiluminescent HRP substrate (Millipore, MA, USA). Intensities of specific bands were normalized to a protein loading control or to the total protein levels marked using MemCode ${ }^{\mathrm{TM}}$ Reversible Protein (Thermo Fisher Scientific, MA, USA). 


\section{Isolation of extracellular vesicles}

Isolation of ectosomes and exosomes was performed using an adapted protocol from previous studies [317, 497]. HEK 293 cells were grown in conditioned medium (depleted of FBS-derived exosomes), produced as previously described [497]. Briefly, DMEM (PAN Biotech, Aidenbach, Germany) supplemented with 20\% FBS (Anprotec, Bruckberg, Germany) and $2 \%$ penicillin-streptomycin (PAN Biotech, Aidenbach, Germany) was centrifuged in polypropylene tubes (Optiseal; Beckman Coulter, CA, USA) in a fixed rotor (type $70 \mathrm{Ti}$; Beckman Coulter, CA, USA) during $16 \mathrm{~h}$ at $100000 \mathrm{xg}, 4^{\circ} \mathrm{C}$. The depleted medium was then diluted with DMEM medium (PAN Biotech, Aidenbach, Germany) in order to reach the final supplements concentration required to make the conditioned medium. The cells were seeded in $T 75 \mathrm{~cm}^{2}$ flasks (Corning, Merck, Darmstadt, Germany) and grew until 80\% confluency, then were washed with warm 1x PBS (PAN Biotech, Aidenbach, Germany) and incubated with $14 \mathrm{~mL}$ of fresh conditioned media during $24 \mathrm{~h}$. The media was collected and placed on ice before centrifuging for $10 \mathrm{~min}$ at $300 \mathrm{xg}$ at $4^{\circ} \mathrm{C}$ to pellet cell debris. The supernatant was collected for new tubes and frozen in $13 \mathrm{~mL}$ aliquots at $-80^{\circ} \mathrm{C}$ for further analyses. To isolate EVs, the supernatant was thawed in ice and centrifuged $20 \mathrm{~min}$ at $2000 \mathrm{xg}, 4^{\circ} \mathrm{C}$. All the isolation protocol was performed in the cold room at $4^{\circ} \mathrm{C}$ and the samples were maintained in ice. $12 \mathrm{~mL}$ of the supernatant was transferred into ultra-clear tubes (Beckman Coulter, CA, USA) and centrifuged in a swing rotor (TH-641 Sorvall; Thermo Fisher Scientific, MA, USA) during $90 \mathrm{~min}$ at $20000 \mathrm{xg}, 4^{\circ} \mathrm{C} .11 \mathrm{~mL}$ of the medium was carefully transferred into a new centrifuge tube with a sterile pipette and the pellet containing ectosomes was resuspended in ice cold 1x PBS (PAN Biotech, Aidenbach, Germany). The medium was again centrifuged in a swing rotor (TH-641 Sorvall; Thermo Fisher Scientific, MA, USA) during 90min at $100000 \times \mathrm{g}$ $\left(4^{\circ} \mathrm{C}\right)$ to generate exosomes. The supernatant was discarded and the pellet containing exosomes was resuspended in ice cold 1x PBS (PAN Biotech, Aidenbach, Germany) with protease and phosphatase inhibitors (cOmplete ${ }^{\mathrm{TM}}$ protease inhibitor and PhosSTOPTM phosphatase inhibitor; Roche, Basel, Switzerland). Both ectosomes and exosomes pellets were again centrifuged at the correspondent velocities in order to concentrate and clean the pellets. Afterwards the supernatant was removed completely by inverting the tubes and the pellets were resuspended in 100 $\mu$ l of 1x PBS (PAN Biotech, Aidenbach, Germany). Protein concentrations were determined by the BCA Protein assay (Thermo Fisher Scientific, MA, USA).

\section{Labelling of the EVs}

Labelling of EVs was performed as previously described [521]. Briefly, Alexa Fluor 633

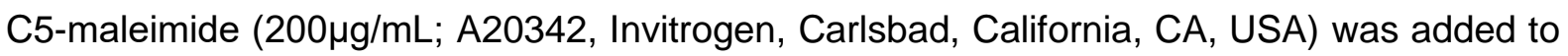


an aliquot of EVs purified after ultracentrifugation containing $60-100 \mu g$ of total protein for a final volume of $50 \mu \mathrm{L}$ in 1x PBS (PAN Biotech, Aidenbach, Germany). Samples were incubated for $1 \mathrm{~h}$ with no agitation in the dark at RT. Non-incorporated and excess of dye was removed by washing the sample in 1x PBS (PAN Biotech, Aidenbach, Germany) and centrifugation during $90 \mathrm{~min}$ at $20000 \mathrm{xg}\left(4^{\circ} \mathrm{C}\right)$ for ectosomes and at $100000 \mathrm{xg}$ for $90 \mathrm{~min}$ for exosomes $\left(4^{\circ} \mathrm{C}\right)$ in a swing rotor (TH-641 Sorvall; Thermo Fisher Scientific, MA, USA). As a control, 1x PBS (PAN Biotech, Aidenbach, Germany) without EVs was also incubated with the dye and performed in parallel to confirm dye removal by washing with 1x PBS (PAN Biotech, Aidenbach, Germany) and ultracentrifugation. For microscopy analysis, labelled EVs were gently mixed with mowiol and applied into PLO-coated coverslips and glass slides.

\section{Sucrose gradient}

Sucrose gradient centrifugation was performed from crude vesicle pellets adapted from previous studies $[497,538]$. Sucrose stock solutions $(10,16,22,28,34,40,46,52,58$, 64, 70 and 90\%) (Sigma-Aldrich, MO, USA) were prepared in 1x PBS (PAN Biotech, Aidenbach, Germany). The crude exosome and ectosome preparations (in 100 $\mu \mathrm{L}$ PBS) were resuspended in $1 \mathrm{~mL}$ of $90 \%$ sucrose stock solution ( $82 \%$ final sucrose concentration). Each sample was transferred to an ultra-clear centrifuge tube (Beckman Coulter, CA, USA) and overlayed with the rest of the gradient stocks on top starting with $1 \mathrm{~mL}$ of $70 \%$ sucrose solution and the last with $10 \%$ solution, in order to make a gradient going from the highest to lowest sucrose concentration. Samples were centrifuged for $16 \mathrm{~h}$ at $100000 \times \mathrm{g}$ at $4^{\circ} \mathrm{C}(\mathrm{TH}-641$ Sorvall; Thermo Fisher Scientific, MA, USA) and afterwards $1 \mathrm{~mL}$ fractions were collected starting from the top to bottom. Fractions were resuspended in 1x PBS (PAN Biotech, Aidenbach, Germany) and centrifuged $100000 \times g$ at $4^{\circ} \mathrm{C}$ for $90 \mathrm{~min}$ (TH-641 Sorvall; Thermo Fisher Scientific, MA, USA). The final pellets were resuspended in 1x PBS (PAN Biotech, Aidenbach, Germany) and applied into a DotBlot system with a $0.2 \mu \mathrm{m}$ nitrocellulose membrane (Bio-Rad, CA, USA).

\section{NTA analysis}

Particle number and size distribution in ectosomes and exosomes samples were determined by nanoparticle tracking analysis (NTA) using a NanoSight LM10 instrument and a LM14 viewing unit equipped with a $532 \mathrm{~nm}$ laser (NanoSight, Salisbury, UK). Total EVs samples were diluted in 1x PBS (PAN Biotech, Aidenbach, Germany) to a final volume of $400 \mathrm{~mL}$ prior to analysis, according to the manufacturer recommendations. Data was recorded using NTA 2.3 software with a detection threshold of 5, captured with a camera level of 16 at 
$25^{\circ} \mathrm{C}$, in videos of $5 \times 60$ seconds (s) repeated 4 times and averaged for each biological replicate.

\section{Electron microscopy}

Electron microscopy images from ectosomes and exosomes was performed following a protocol previously described [497]. Isolated EVs were bound to a glow discharged carbon foil covered grids. After staining with $1 \%$ uranyl acetate ${ }_{(a q .)}$, the samples were evaluated at RT with a Talos L120C transmission electron microscope (Thermo Fisher Scientific, MA, USA).

\section{Proteomic analyses of EVs}

Samples were resuspended in Laemmli sample buffer and separated by SDS-PAGE. The entire lane was subsequently cut in 23 gel pieces and tryptically digested, as previously described [539]. Peptides extracted from the gel pieces were analysed in technical triplicates by liquid chromatography coupled to mass spectrometry (LC-MS) on a Dionex UltiMate 3000 RSLCnano system connected to an Orbitrap Fusion mass spectrometer (Thermo Fisher Scientific, MA, USA). Peptides were separated by a 43 min gradient ranging from $8 \%$ to $37 \%$ acetonitrile on an in-house packed C18 column $(75 \mu \mathrm{m} \times 30 \mathrm{~cm}$, Reprosil-Pur 120C18-AQ, $1.9 \mu \mathrm{m}$, Dr. Maisch $\mathrm{GmbH}$, Ammerbuch, Germany) at $300 \mathrm{nl} / \mathrm{min}$ flow rate. MS1 spectra were acquired with 120000 resolution (full width at half maximum, FWHM) and a scan range from 350 to $1,600 \mathrm{~m} / \mathrm{z}$. Within a cycle time of $3 \mathrm{~s}$, precursor ions with a charge state between +2 and +7 were selected individually with a $1.6 \mathrm{~m} / \mathrm{z}$ isolation window and were fragmented with a normalized collision energy of 35 by higher energy collisional dissociation (HCD). MS2 spectra were acquired in the ion trap with $20 \%$ normalized AGC and dynamic injection time. Once selected precursors were excluded from another fragmentation event for 30s. Raw acquisition files were subjected to database search with Maxquant (version 1.6.2.10) [540] against the reference proteome of Homo sapiens (downloaded on 19/2019). Default settings were used unless stated differently below. Fractions were defined according to the cutting of the gel lanes and experiments were defined on the level of technical replicates. Unique and razor peptides were used for label-free quantification.

Data analysis was performed with Perseus (version and 1.6.15.0) [541]. Reverse hits, potential contaminants and hits only identified by site were filtered out. Quantitative values were averaged across technical replicates ignoring missing values. A two-sample t-test was performed on biological replicates of ectosomes and exosomes with an artificial within groups variance (s0) of 0.1 and a permutation-based FDR of 0.1 for multiple testing correction. Results were visualized by volcano plots. To assess the reproducibility of the experiments 
within the biological replicates of each cell extracellular vesicle type, we employed principal component analysis (PCA), which was performed using the Perseus built-in tool. The EVs subtype-signature proteins were selected by extracting in Perseus the proteins with the most distinct expression profiles between the different subtypes. For hierarchical clustering, normalized intensities were first z-scored and then clustered using Euclidean as a distance measure for column and row clustering. GO enrichment analyses were performed using Perseus software and the human proteome as background ("mainAnnot.homosapiens.txt.gz, downloaded in 04/2021). Enrichment was considered statistically significant using Fisher exact test and corrected for multiple testing by the Benjamini-Hochberg FDR method with adjusted 0.02 threshold value (GO terms were plotted as -log10 of the p value). Gene ontology analyses were performed using the hit proteins identified in each EVs type.

\section{Internalization experiment with EVs}

Primary cortical neurons were treated with $20 \mu \mathrm{g} / \mathrm{mL}$ of ectosomes or exosomes resuspended in 1x PBS (Sigma-Aldrich, MO, USA). EVs were added to cortical neurons at DIV14 and collected for further analyses at DIV15, 24h after the treatment.

\section{Lactate DeHydrogenase assay}

Cytotoxicity in primary and cell line cultures was assessed using the cytotoxicity Lactate DeHydrogenase (LDH) detection kit according to the manufacturer's instructions (Roche, Basel, Switzerland). The culture medium was centrifuged at $500 \times \mathrm{g}$ for $5 \mathrm{~min}$ to pellet cell debris before used in the experiments.

\section{Multi-electrode array}

Multi-electrode array (MEA) experiments were performed following standard protocols for MEA recordings [523-526, 542, 543]. Primary cortical neuronal cultures were plated directly on 60MEA200/30iR-Ti-gr planar arrays (60 electrodes, 30 $\mu \mathrm{m}$ electrode diameter and $200 \mu \mathrm{m}$ electrode spacing; MultiChannel Systems, Reutlingen, Germany). The arrays were coated with PLL $\left(500 \mu \mathrm{g} / \mathrm{mL}\right.$ in borate buffer; Sigma-Aldrich, MO, USA) overnight at $4^{\circ} \mathrm{C}$. The arrays were rinsed three times with distilled water before coating with laminin $(5 \mu \mathrm{g} / \mathrm{mL}$ in distilled water; Sigma-Aldrich, MO, USA) for at least $1 \mathrm{~h}$ at RT. The solution was aspirated, and the cells were directly plated on top of the electrodes. Neurons were treated with $20 \mu \mathrm{g} / \mathrm{mL}$ of EVs at DIV14 and recorded at DIV15, 24h after the treatment. The neuronal activity over the array was recorded using the MultiChannel MEA2100 system (MultiChannel Systems, Reutlingen, Germany) and the cells were kept in their culture medium with temperature maintained at 35- 
$37^{\circ} \mathrm{C}$ during recordings. To avoid movement-induced artifacts, recordings started $10 \mathrm{~min}$ after translocation of arrays from the incubator to the recording stage. The spontaneous activity of the cultured neurons was recorded for 5 - $10 \mathrm{~min}$. The electrode signals were amplified, bandpass filtered $(200 \mathrm{~Hz}$ to $3 \mathrm{kHz})$ and recorded digitally at $25 \mathrm{kHz}$, using the MultiChannel Experimenter software (version 2.17.7.0, MultiChannel Systems, Reutlingen, Germany).

Spike sorting was carried out using a modified version of the Kilosort automatic sorting software [544, 545] (available at: https://github.com/MouseLand/Kilosort and https://github.com/dimokaramanlis/KiloSortMEA). The output of Kilosort was visually inspected and manually curated with the "Phy 2" software (https://github.com/cortex-lab/phy). Only those clusters of spikes ("units") with a well-separated spike waveform and a clear refractory period were included in the final analysis and considered as coming from individual cells. The spike clusters were pre-processed and analysed using custom-made MATLAB scripts (Version: 9.7.0, R2019b; Mathworks, MA, USA). The raster plots, average firing rate and spike amplitude were measured from the spontaneous activity of each recorded cell. In all our recordings, we observed frequent "bursts" - groups of spikes occurring rapidly and consecutively with short inter-spike intervals [less than few tens of milliseconds (ms)], followed by quiescent periods much longer than typical inter-spike intervals (in our recordings generally several seconds). The bursts typically occurred synchronously for multiple cells over the array and in our analysis, we focused on such population-wide synchronized bursts. To detect the concurrent bursts, we computed the population firing rate as a histogram (100ms bin size) of array-wide spiking activity. The peaks of the firing rate histogram were used to detect synchronous, array-wide bursts with at least $500 \mathrm{~ms}$ distance between two consecutive peaks. The peaks that were smaller than $1 / 5$ of the largest peak were excluded as they do not correspond to array-wide, synchronous activity. A time window of $650 \mathrm{~ms}$ around each peak (150ms before to $500 \mathrm{~ms}$ after) was defined as the burst window (onset and offset of each burst). For each recorded cell, the spikes belonging to bursts were measured during the defined burst windows, and cells with fewer than six spikes across all their detected bursts were excluded from this analysis. From the detected bursts, the following parameters were calculated: (1) burst rate of the culture as the number of bursts per time over the duration of each recording; (2) inter-burst-interval as the time between the measured offset of a burst and the onset of the following burst, calculated for each pair of successive bursts in a recording; (3) burst duration for each cell as the time between the cell's first and last spike during the burst window; (4) intra-burst-frequency as the rate of spikes occurring within a burst, averaged over all the detected bursts for each cell; and (5) percentage of spikes in bursts as the ratio of spikes occurring during bursts relative to the total number of spikes for each cell. 


\section{Confocal imaging}

Imaging was performed on a Leica SP5 confocal laser scanning microscope equipped with hybrid detectors using Application Suite $X$ software with 100x immersion objective lenses (Leica Biosystems, Wetzlar, Germany). Samples were excited using 405 Diode, argon and helium-neon 633 lasers, pinhole $=1,0.2 \mu \mathrm{m}$ thickness $Z$ stacks and 2 averaging line-by-line. The acquisition settings were optimized to avoid underexposure and oversaturation effects and kept equal throughout image acquisition of the samples.

\section{STED microscopy}

Samples were imaged using an inverse 4-channel Expert Line easy3D STED setup (Abberior Instruments, Göttingen, Germany). The setup was based on an Olympus IX83 microscope body equipped with a plan apochromat 100x 1.4 NA oil-immersion objective (Olympus). Fluorescence lasers $595 \mathrm{~nm}$ and $775 \mathrm{~nm}$ (Abberior Instruments, Göttingen, Germany) were utilized for the imaging. Fluorescence signal was detected using avalanche photodiodes detectors (Abberior Instruments, Göttingen, Germany) in predefined channels. The operation of the setup and the recording of images were performed with the Imspector software, version 0.14 (Abberior Instruments, Göttingen, Germany).

\section{Quantification and statistical analysis}

Images were analysed using ImageJ software (National Institutes of Health) [546]. To analyse the degree of EVs uptake in neuronal cells, measurement of EVs and cell area was performed from isolated areas chosen randomly within regions containing EVs signal. All data are presented as mean \pm SD. Data from at least three independent experiments and each replicate represents one independent experiment. To assess differences between two groups, two-tailed unpaired student t-test was performed using GraphPad Prism 9 software (GraphPad, CA, USA). To assess differences between more than two groups, significant differences were assessed by one-way ANOVA followed by multiple comparisons with significance between groups corrected by Bonferroni procedure using GraphPad Prism 9 software (GraphPad, CA, USA). Differences were considered to be significant for values of $\mathrm{p}<0.05$ and are expressed as mean $\pm \mathrm{SD}$. For mass spectrometry, the spectral count differences between samples were considered to be significant for FDR values $<0.1$ (see proteomic analyses section). 


\section{Supplementary Figures}

A

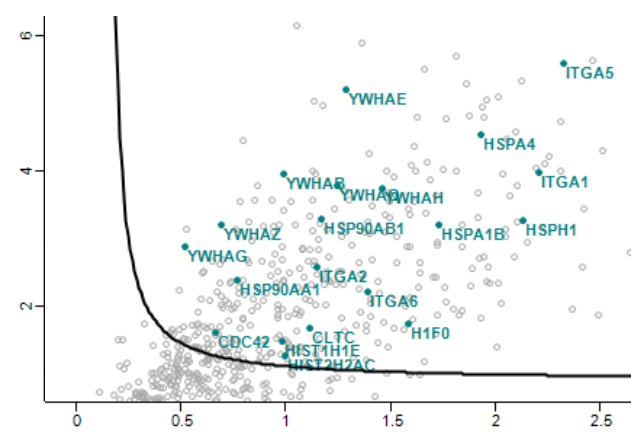

B

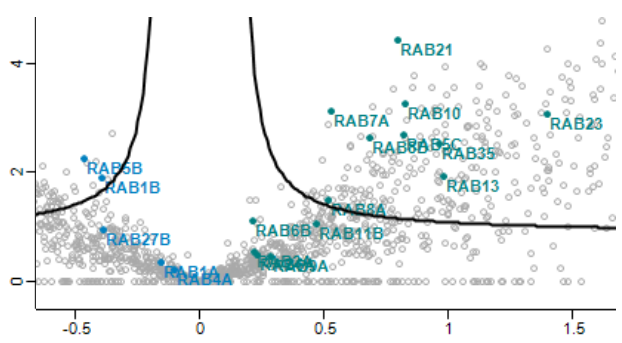

Supplementary Figure 1. Protein hits enriched in ectosomes and exosomes. Volcano plots of quantitative differences in proteins in EVs fractions. (A) Other proteins enriched in ectosomes - clathrin (CLTC), cell division control protein 42 (CDC42), integrin alpha-1/ 2/ 5/ 6 (ITGA1/ 2/ 5/ 6), 14-3-3 proteins (YWHAZ/ E/ B/ G/ H/ Q), histones H1.4 / H1.0/ H2A (HIST1H1E / H1FO/ HIST2H2AC), heat shock protein HSP90 (HSP9OAB1/ AA1), heat shock $70 \mathrm{kDa}$ protein (HSPA1A/ B, HSPA4) and heat shock protein $105 \mathrm{kDa}$ (HSPH1). (B) Ras-related proteins (Rabs) identified in EVs - in exosomes Rab-1A/ 1B/4A/5B/27B (RAB1A/ 1B/4A/5B/27B), in ectosomes Rab-2A/ $5 \mathrm{C} / 6 \mathrm{~A} / 6 \mathrm{~B} / \mathrm{7A} / 8 \mathrm{~A} / 8 \mathrm{~B} / 9 \mathrm{~A} / 10 / 11 \mathrm{~B} / 13 / 21 / 23 / 35$ (RAB2A/5C/ 6A/ 6B/ 7A/ 8A/ 8B/ 9A/ 10/ 11B/ 13/21/ 23/35). Blue dots represent the proteins enrichment in exosomes, while turquoise dots represent enrichment in ectosomes. Proteins in the plots are identified with their gene name. Dots above the dashed line represent proteins for which differences were significant (false discovery rate $[F D R]<0.1$ ). Data represented in "t-test Difference (Ectosomes Exosomes)" vs. "-Log t-test p-value" from five independent samples for each group. Data analyses were performed using Perseus software. 


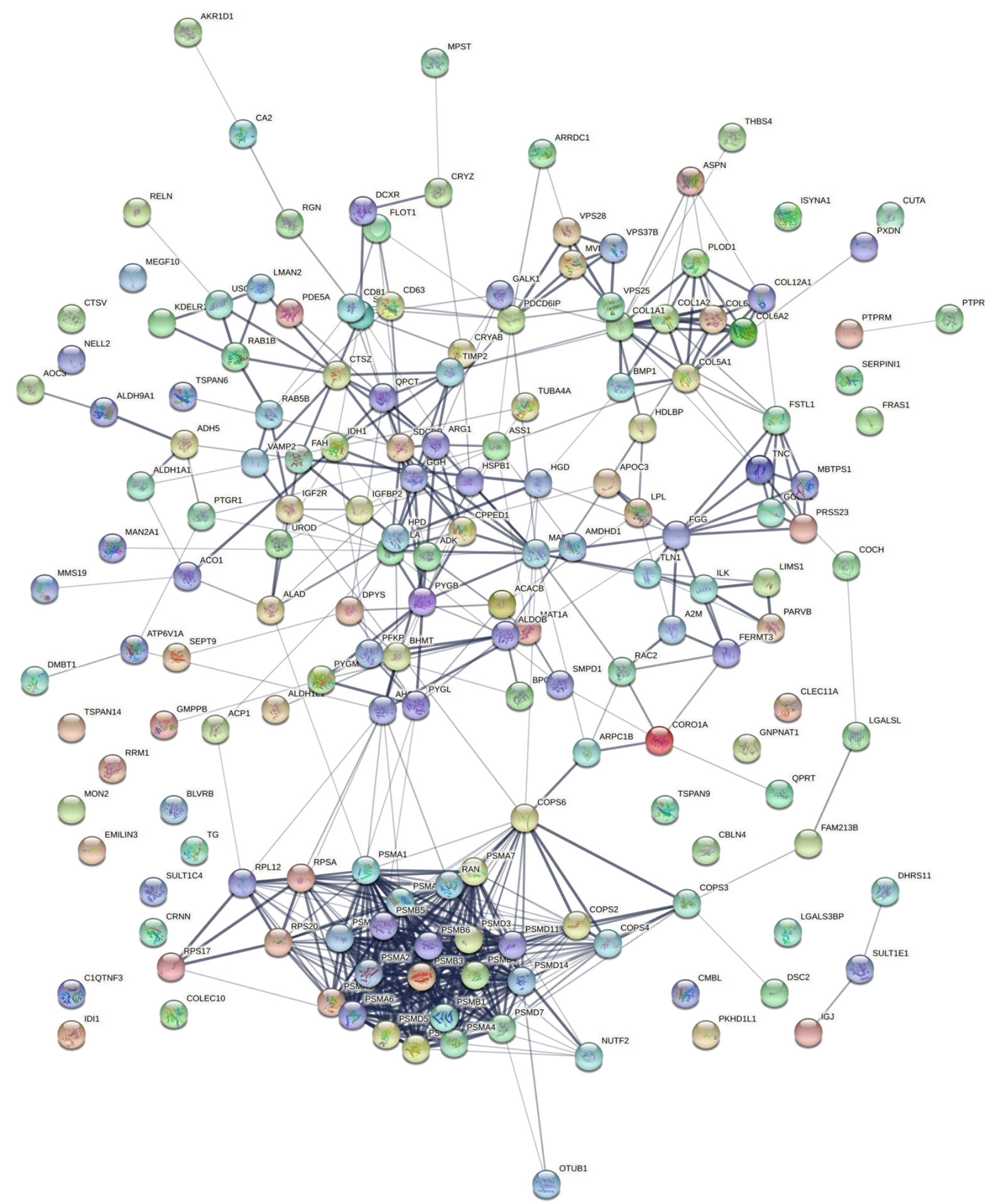

Supplementary Figure 2. Functional protein association networks were plotted by using the STRING database. The exosomal proteins significantly identified in our study show robust networks and significant overlap. The line thickness represents the confidence of the association (https://version-11-0b.stringdb.org/cgi/network?networkld=br68rv2GDNdl ). 


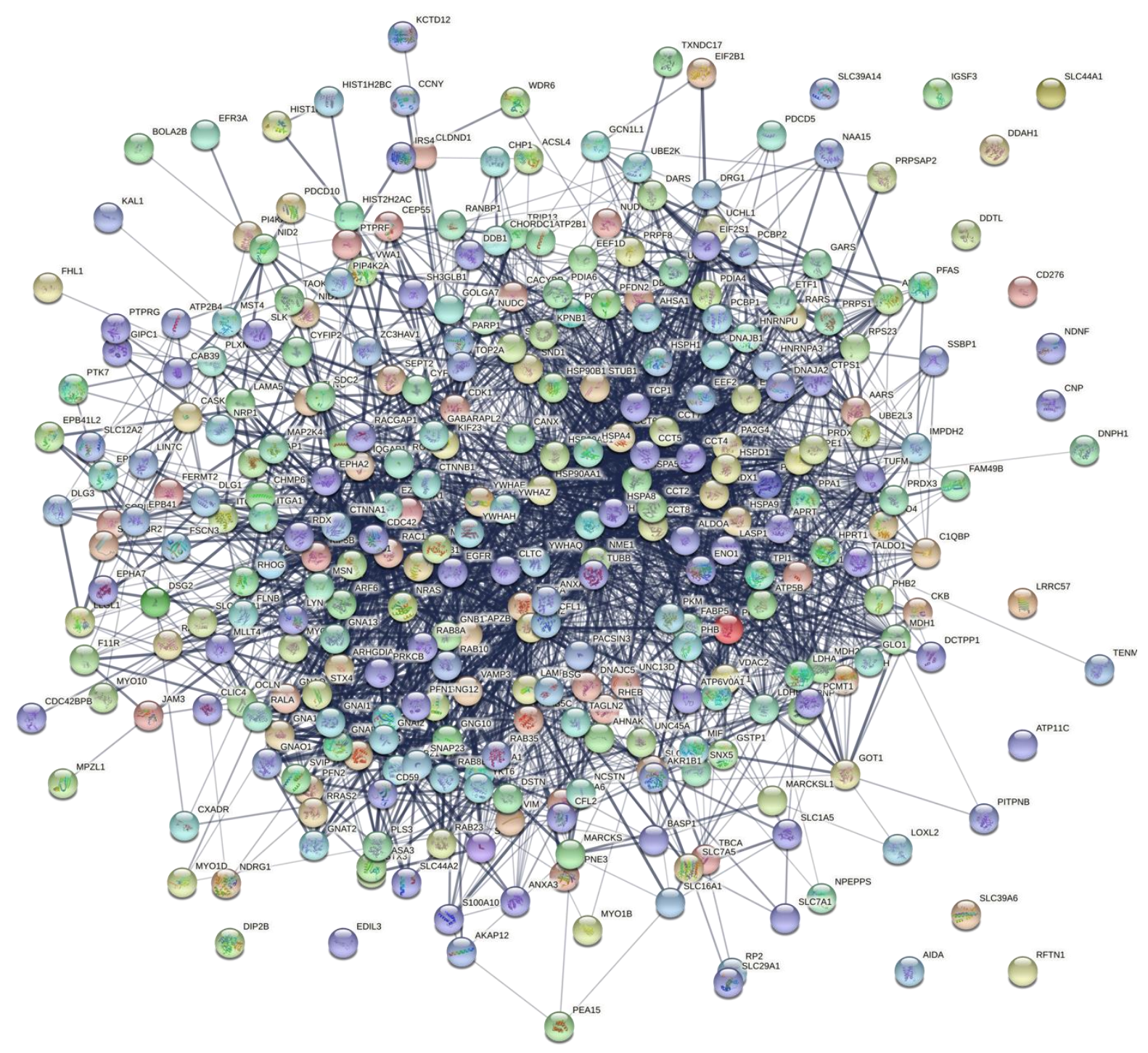

Supplementary Figure 3. Functional protein association networks were plotted by using the STRING database. The ectosome proteins significantly identified in our study show robust networks and significant overlap. The line thickness represents the confidence of the association (https://version-11-0b.stringdb.org/cgi/network?networkld=bwoR4jPEXhpC ). 
A

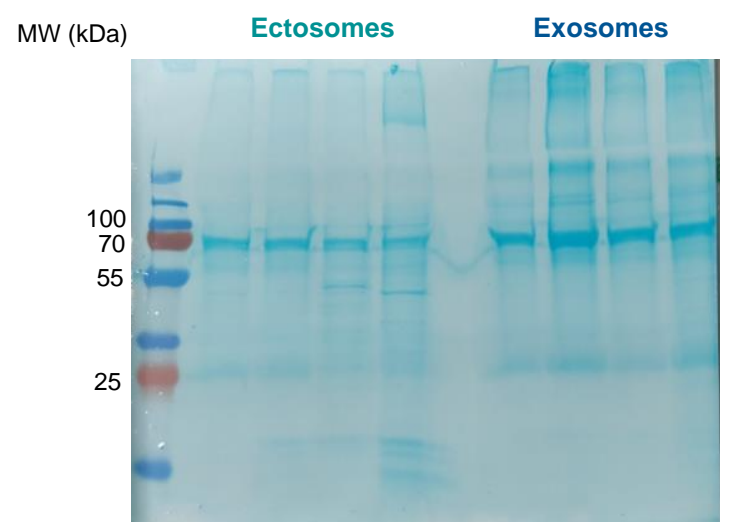

B

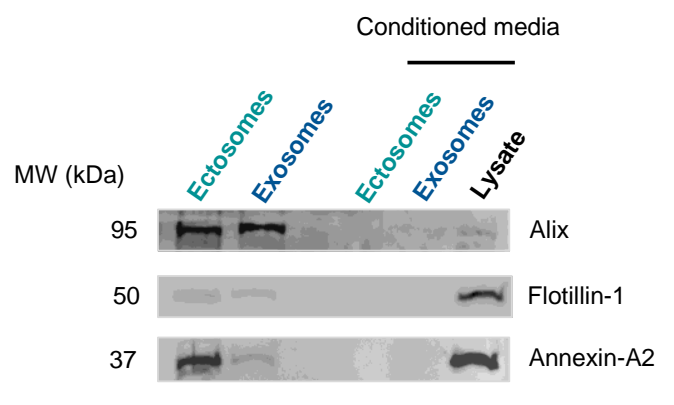

Conditioned media

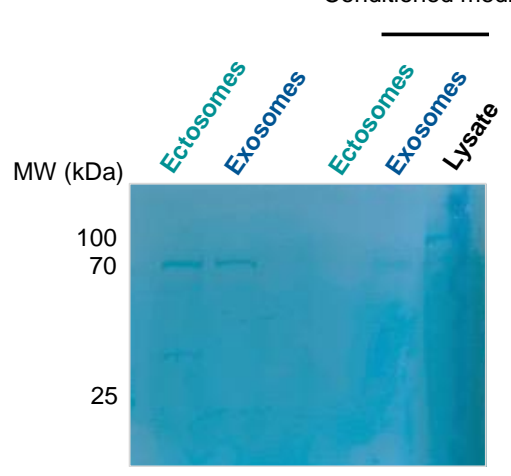

Supplementary Figure 4. Conditioned media used for the experiments does not contain residual EVs. (A) Memcode staining from four different replicates from ectosomes and exosomes pellets purified from different media collections showing the efficiency in the isolation protocol. (B) Immunoblot of ectosomes and exosomes released by HEK cells, EVs that are present in the conditioned cell media without incubation with the cells and whole-cell lysate. Equal quantities of protein were separated on SDS-PAGE gels, and membranes were blotted for alix, flotillin1 and Annexin-A2.

A
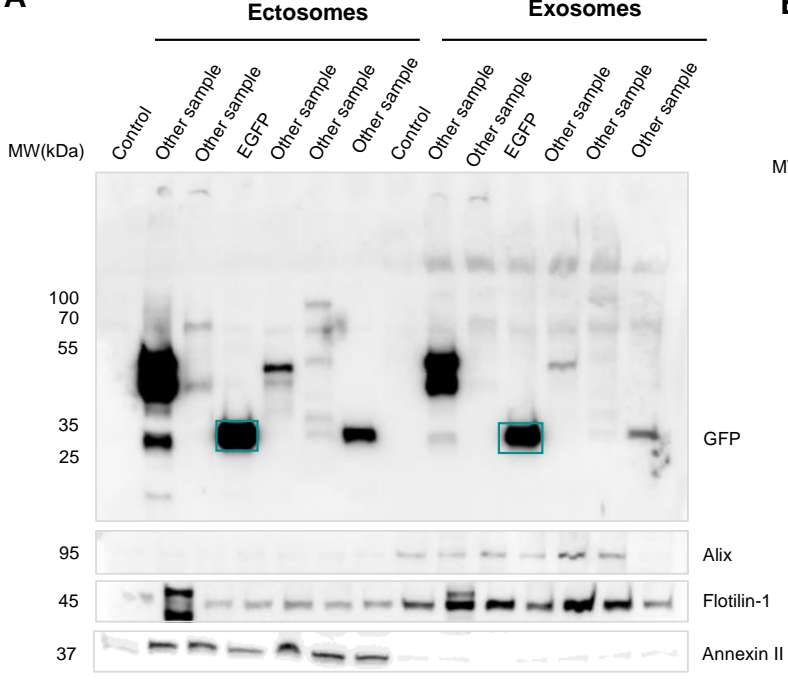

B

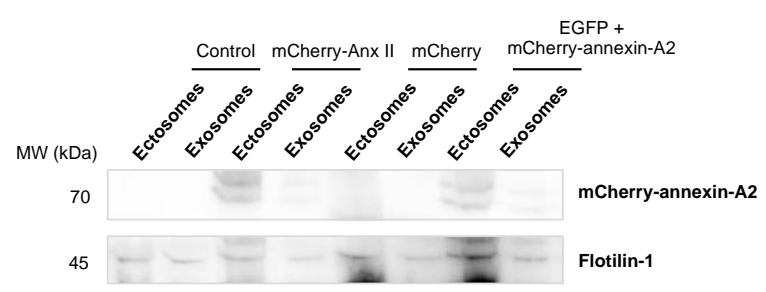

Supplementary Figure 5. Characterization of EGFP and annexin-A2 levels in EVs. (A) Immunoblot showing the levels of EGFP in ectosomes and exosomes and several EVs markers. (B) Immunoblot displaying the levels of mCherry-Annexin-A2 and flotillin-1 in EVs. 
A

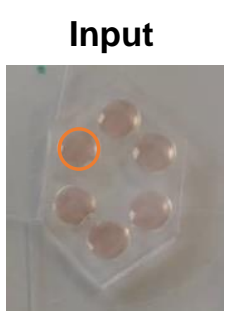

B

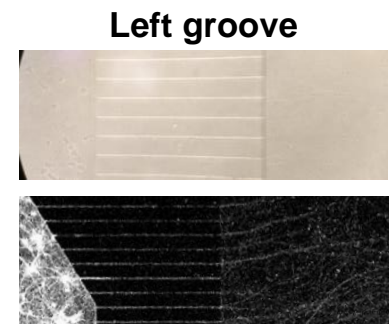

Middle groove

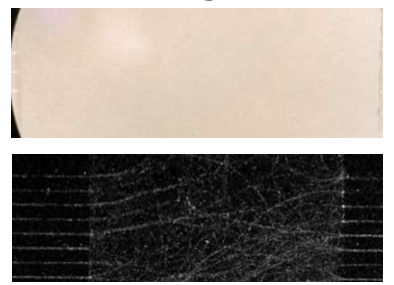

Right groove

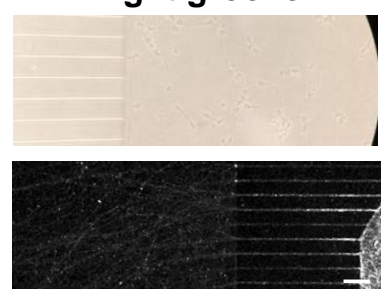

C
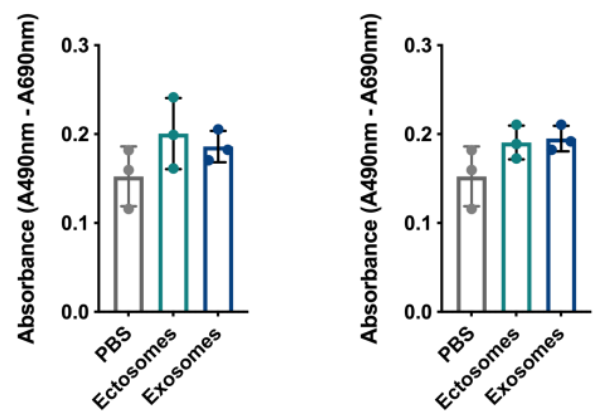

Supplementary Figure 6. Cellular uptake of EVs by primary cortical neurons. (A) Primary cortical neurons were seeded in microfluidic devices to separate neuronal cell bodies. EVs containing EGFP were added to primary cortical neurons at DIV14 with $20 \mu \mathrm{g} / \mathrm{mL}$ of ectosomes or exosomes for 24 hours in the upper left well of the device. (B) Top panel shows contrast light images of the primary cortical neurons growing in microfluidic devices. Bottom panel shows neurons cell bodies and axons labelled with membrane cell dye Alexa Fluor 633 C5-maleimide (scale bar $100 \mu \mathrm{m})$. (C) Lactate DeHydrogenase (LDH) measurements show that EVs enriched with EGFP or labelled with dye show similar low toxicity for the primary cortical neurons. 
A

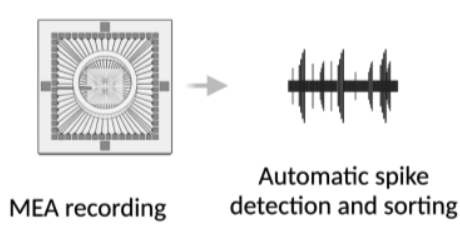

C

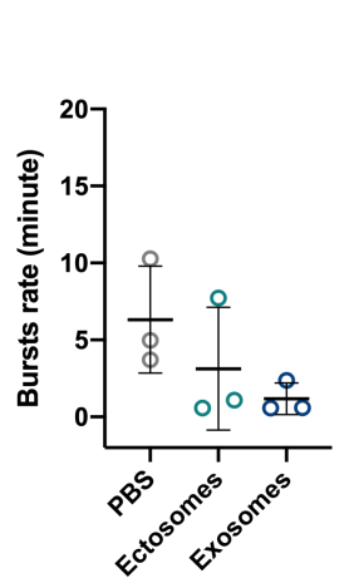

B

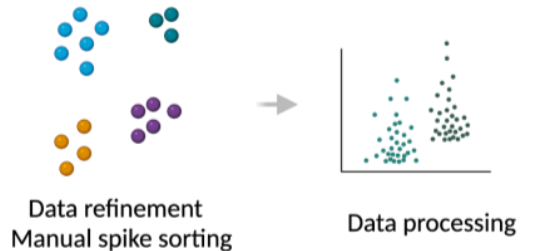

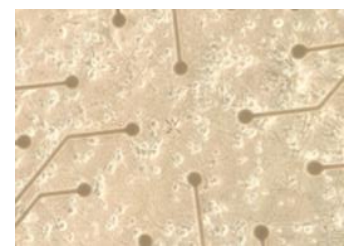

D

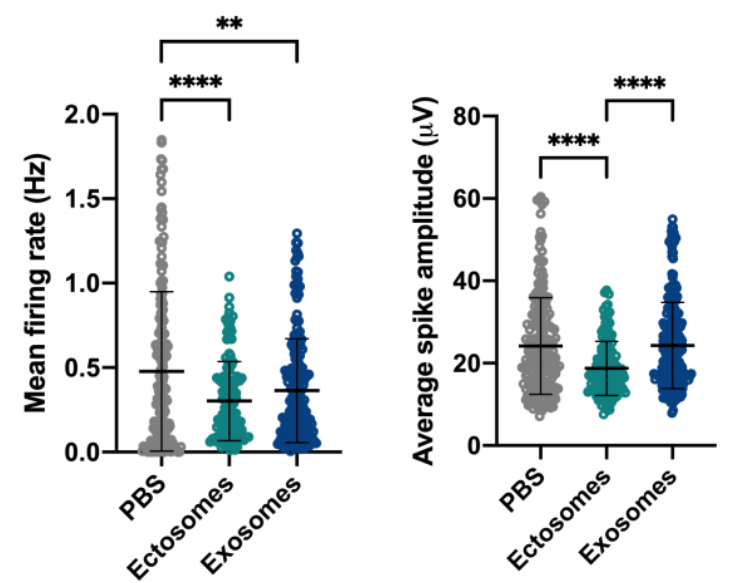

Supplementary Figure 7. EVs modulate spontaneous activity in primary cortical neurons. (A) MEA recordings were used to monitor neural spike firing after treating primary cortical neurons with $20 \mu \mathrm{g} / \mathrm{mL}$ of ectosomes or exosomes at DIV14 for 24 hours. Schematic representation shows the steps performed for the data analysis. (B) Phase-contrast image of primary cortical neurons cultured on an MEA chamber. (C) Bursts rate (per minute) of the cortical neurons treated with EVs. (D) Quantification of the mean firing rate and average spike amplitude of primary cortical neurons incubated with PBS, ectosomes or exosomes. Data from at least three independent experiments for each condition. Significant differences were assessed by one-way ANOVA followed by multiple comparisons with significance between groups corrected by Bonferroni procedure. Differences were considered to be significant for values of $p<0.05$ and are expressed as mean $\pm S D,{ }^{*} p<0.05,{ }^{* *} p<0.01$. 


\section{Supplementary Tables}

Supplementary Table 1. List of significantly altered proteins identified in exosomes. Common proteins (11) in the list of Top 100 proteins often identified in EVs are highlighted in bold (see Supplementary Table 3).

Protein names

\begin{tabular}{|c|c|c|}
\hline Alix & PDCD6IP & 4,836 \\
\hline Glycogen phosphorylase, muscle form & PYGM & 4,300 \\
\hline Proteasome subunit beta type-1 & PSMB1 & 4,098 \\
\hline Glycogen phosphorylase, liver form & PYGL & 4,097 \\
\hline Proteasome subunit alpha type-7 & PSMA7 & 3,984 \\
\hline Proteasome subunit beta type-7 & PSMB7 & 3,752 \\
\hline Asporin & $A S P N$ & 3,734 \\
\hline Proteasome subunit alpha type- 6 & PSMA6 & 3,695 \\
\hline Proteasome subunit beta type- 5 & PSMB5 & 3,652 \\
\hline Homogentisate 1,2-dioxygenase & $H G D$ & 3,611 \\
\hline Receptor-type tyrosine-protein phosphatase & PTPRM & 3,610 \\
\hline Vacuolar protein sorting-associated protein 28 homolog & VPS28 & 3,525 \\
\hline Proteasome subunit alpha type-2 & PSMA2 & 3,511 \\
\hline Proteasome subunit alpha type-4 & PSMA4 & 3,460 \\
\hline Proteasome subunit beta type- 4 & PSMB4 & 3,443 \\
\hline Cation-independent mannose-6-phosphate receptor & IGF2R & 3,377 \\
\hline Eukaryotic initiation factor $4 \mathrm{~A}-\mathrm{II}$ & EIF4A2 & 3,345 \\
\hline Protein kinase C-binding protein NELL2 & NELL2 & 3,340 \\
\hline 3-mercaptopyruvate sulfurtransferase & MPST & 3,327 \\
\hline Mannose-1-phosphate guanyltransferase beta & GMPPB & 3,264 \\
\hline Cytosolic 10 -formyltetrahydrofolate dehydrogenase & ALDH1L1 & 3,226 \\
\hline Cytoplasmic aconitate hydratase & ACO1 & 3,172 \\
\hline S-adenosylmethionine synthase isoform type-1 & MAT1A & 3,097 \\
\hline Delta-aminolevulinic acid dehydratase & $A L A D$ & 3,039 \\
\hline Tubulin alpha-4A chain & TUBA4A & 3,033 \\
\hline Vacuolar protein sorting-associated protein 37B & VPS37B & 3,012 \\
\hline Adenosylhomocysteinase & $A H C Y$ & 2,989 \\
\hline Regucalcin & $R G N$ & 2,989 \\
\hline Fructose-bisphosphate aldolase $\mathrm{B}$ & $A L D O B$ & 2,989 \\
\hline 4-trimethylaminobutyraldehyde dehydrogenase & $A L D H 9 A 1$ & 2,960 \\
\hline Microtubule-associated protein RP/EB family member 2 & MAPRE2 & 2,933 \\
\hline Uroporphyrinogen decarboxylase & UROD & 2,927 \\
\hline Coronin-1A & CORO1A & 2,918 \\
\hline Serine protease 23 & PRSS23 & 2,896 \\
\hline Proteasome subunit beta type-2 & PSMB2 & 2,860 \\
\hline Haptoglobin & $H P$ & 2,859 \\
\hline Collagen alpha-3(VI) chain & COL6A3 & 2,814 \\
\hline Argininosuccinate synthase & ASS1 & 2,791 \\
\hline Galectin-3-binding protein & LGALS3BP & 2,774 \\
\hline von Willebrand factor;von Willebrand antigen 2 & $V W F$ & 2,736 \\
\hline Lipoprotein lipase & $L P L$ & 2,730 \\
\hline Protein MON2 homolog & MON2 & 2,712 \\
\hline Coagulation factor $X$ & F10 & 2,700 \\
\hline
\end{tabular}


3-oxo-5-beta-steroid 4-dehydrogenase

Proteasome subunit alpha type-5

Reelin

Proteasome subunit alpha type-3

Prostaglandin reductase 1

Complement C4-A

Prostamide/prostaglandin F synthase

Collectin-10

26S proteasome non-ATPase regulatory subunit 5

Carbonic anhydrase 2

Ferritin heavy chain

COP9 signalosome complex subunit 7b

Clusterin

COP9 signalosome complex subunit 3

Syntenin-1

Immunoglobulin $\mathrm{J}$ chain

Retinal dehydrogenase 1

Ectonucleotide pyrophosphatase/phosphodiesterase family member 2

\begin{tabular}{|l|} 
AKR1D1 \\
\hline PSMA5 \\
RELN \\
\hline PSMA3
\end{tabular}

Alpha-crystallin B chain

Integrin-linked protein kinase

Ubiquitin thioesterase OTUB1

Tenascin

Neuroserpin

Quinone oxidoreductase

Prolow-density lipoprotein receptor-related protein 1

Proteasome subunit beta type-6

Membrane-bound transcription factor site-1 protease

Proteasome subunit alpha type-1

General vesicular transport factor p115

Alcohol dehydrogenase class-3

COP9 signalosome complex subunit 4

Sulfotransferase 1C2

Thyroglobulin

Ras-related protein Rab-5B

Collagen alpha-1(II) chain

Tetraspanin-14

Betaine--homocysteine S-methyltransferase 1

Collagen alpha-2(V) chain

Thrombospondin-4

Proteasome subunit beta type-3

Nicotinate-nucleotide pyrophosphorylase [carboxylating]

Golgi membrane protein 1

Flavin reductase (NADPH)

$26 \mathrm{~S}$ protease regulatory subunit $6 \mathrm{~A}$

Phenazine biosynthesis-like domain-containing protein

Glycogen phosphorylase, brain form

Galactokinase

Follistatin-related protein 1

PTGR

C4A;C4B

FAM213B

COLEC10

PSMD5

CA2

FTH1

COPS7B

CLU

COPS3

SDCBP

IGJ

ALDH1A1

ENPP2

$C R Y A B$

ILK

OTUB1

TNC

SERPINI1

CRYZ

LRP1

PSMB6

MBTPS1

PSMA1

USO1

ADH5

COPS4

SULT1C2

$T G$

RAB5B

COL2A1

TSPAN14

BHMT

COL5A2

THBS4

PSMB3

QPRT

GOLM1

BLVRB

PSMC3

PBLD

PYGB

GALK1

FSTL1
2,696

2,660

2,654

2,636

2,592

2,588

2,553

2,547

2,523

2,519

2,515

2,509

2,506

2,467

2,464

2,463

2,440

2,412

2,407

2,402

2,375

2,365

2,360

2,330

2,328

2,324

2,321

2,316

2,311

2,301

2,294

2,293

2,282

2,269

2,246

2,231

2,218

2,204

2,203

2,197

2,184

2,177

2,156

2,154

2,146

2,135

2,130

2,130 
Aflatoxin $\mathrm{B} 1$ aldehyde reductase member 4

Actin-related protein 2/3 complex subunit 1B

Ribonucleoside-diphosphate reductase large subunit

Protocadherin Fat 1

Septin-9

4-hydroxyphenylpyruvate dioxygenase

Complement C1q tumor necrosis factor-related protein 3

Vesicular integral-membrane protein VIP36

Procollagen-lysine,2-oxoglutarate 5-dioxygenase 1

Collagen alpha-1(I) chain

Fumarylacetoacetate hydrolase domain-containing protein $2 \mathrm{~A}$

Ras-related protein Rab-1B

Dehydrogenase/reductase SDR family member 11

Collagen alpha-2(I) chain

GTP-binding nuclear protein Ran

Collagen alpha-1(V) chain

Metalloproteinase inhibitor 2

Tetraspanin-6

Serine/threonine-protein phosphatase 2A catalytic subunit alpha isoform

Mitogen-activated protein kinase 1

Endothelial protein $\mathrm{C}$ receptor

Isocitrate dehydrogenase [NADP] cytoplasmic

Membrane primary amine oxidase

Low molecular weight phosphotyrosine protein phosphatase

Bone morphogenetic protein 1

60S ribosomal protein L12

\begin{tabular}{l|l}
\hline Adenosine kinase & AD \\
\hline Beta-parvin & $P A$ \\
\hline Peroxidasin homolog & $P X$ \\
\hline
\end{tabular}

\begin{tabular}{l|l}
\hline Arginase-1 & $A R$ \\
\hline Dihydropyrimidinase & $D P$
\end{tabular}

Fibrinogen gamma chain

\begin{tabular}{|c|c|c|}
\hline Tetraspanin-9 & TSPAN9 & 1,742 \\
\hline Transforming growth factor beta-1 & TGFB1 & 1,740 \\
\hline Fibrocystin-L & PKHD1L1 & 1,737 \\
\hline Glutaminyl-peptide cyclotransferase & QPCT & 1,720 \\
\hline Fumarylacetoacetase & $F A H$ & 1,715 \\
\hline Arrestin domain-containing protein 1 & $A R R D C 1$ & 1,706 \\
\hline Probable maltase-glucoamylase-like protein & LOC93432 & 1,703 \\
\hline V-type proton ATPase catalytic subunit A & ATP6V1A & 1,700 \\
\hline Alpha-galactosidase A & GLA & 1,698 \\
\hline Probable imidazolonepropionase & $A M D H D 1$ & 1,690 \\
\hline ProSAAS & PCSK1N & 1,685 \\
\hline Cerebellin-4 & CBLN4 & 1,685 \\
\hline Receptor-type tyrosine-protein phosphatase kappa & PTPRK & 1,671 \\
\hline Collagen alpha-2(VI) chain & COL6A2 & 1,671 \\
\hline Lysosomal alpha-mannosidase & MAN2B1 & 1,666 \\
\hline Cathepsin L2 & CTSV & 1,659 \\
\hline
\end{tabular}


Glucosamine 6-phosphate N-acetyltransferase

GNPNAT

Isopentenyl-diphosphate Delta-isomerase 1

Vesicle-associated membrane protein 2

cGMP-specific 3,5-cyclic phosphodiesterase

cAMP-dependent protein kinase type I-alpha regulatory subunit

Acetyl-CoA carboxylase 1

Alpha-mannosidase 2

Heat shock protein beta-1

Extracellular matrix protein FRAS1

CD63 antigen

MMS19 nucleotide excision repair protein homolog

Apolipoprotein C-III

40S ribosomal protein S20

L-xylulose reductase

Fermitin family homolog 3

Prolyl endopeptidase FAP

Estrogen sulfotransferase

Sphingomyelin phosphodiesterase

Ras-related C3 botulinum toxin substrate 2

40S ribosomal protein SA

Sorbitol dehydrogenase

Vacuolar protein-sorting-associated protein 25

Protein CutA

26S proteasome non-ATPase regulatory subunit 14

C-type lectin domain family 11 member $A$

Talin-1

Gelsolin

COP9 signalosome complex subunit 2

Putative RNA-binding protein Luc7-like 2

40S ribosomal protein S17

CD81 antigen

Transmembrane protein 132C

Nuclear transport factor 2

Inositol-3-phosphate synthase 1

26S proteasome non-ATPase regulatory subunit 7

Cochlin

ER lumen protein-retaining receptor 1

ATP-dependent 6-phosphofructokinase, platelet type

Carboxymethylenebutenolidase homolog

26S proteasome non-ATPase regulatory subunit 11

Deleted in malignant brain tumors 1 protein

Pleckstrin homology domain-containing family B member 2

Gamma-glutamyl hydrolase

Complement C3

LIM and senescent cell antigen-like-containing domain protein 1

Complement C1r subcomponent

Desmocollin-3

COP9 signalosome complex subunit 6

IDII

VAMP2

PDE5A

PRKAR1A

ACACA

MAN2A1

HSPB1

FRAS1

CD63

MMS19

APOC3

RPS20

$D C X R$

FERMT3

FAP

SULT1E1

SMPD1

RAC2

RPSA

SORD

VPS25

CUTA

PSMD14

CLEC11A

TLN1

GSN

COPS2

LUC7L2

RPS17

CD81

TMEM132C

NUTF2

ISYNA1

PSMD7

$\mathrm{COCH}$

KDELR1

PFKP

$C M B L$

PSMD11

DMBT1

PLEKHB2

GGH

C3

LIMS1

C1R

DSC3

COPS6 
26S proteasome non-ATPase regulatory subunit 3

\begin{tabular}{|c|c|c|}
\hline Bisphosphoglycerate mutase & BPGM & 1,301 \\
\hline Galectin-related protein & LGALSL & 1,282 \\
\hline Cathepsin Z & CTSZ & 1,275 \\
\hline Tetraspanin-7 & TSPAN7 & 1,272 \\
\hline Multiple epidermal growth factor-like domains protein 10 & MEGF10 & 1,242 \\
\hline EMILIN-3 & EMILIN3 & 1,232 \\
\hline Vigilin & $H D L B P$ & 1,225 \\
\hline Serine/threonine-protein phosphatase CPPED1 & CPPED1 & 1,189 \\
\hline Collagen alpha-1(XII) chain & COL12A1 & 1,181 \\
\hline Alpha-2-macroglobulin & A2M & 1,112 \\
\hline Ig gamma-4 chain $\mathrm{C}$ region & IGHG4 & 1,089 \\
\hline Cornulin & CRNN & 1,037 \\
\hline Hemoglobin subunit beta & $H B B$ & 1,016 \\
\hline
\end{tabular}

Supplementary Table 2. List of significantly altered proteins identified in ectosomes. Common proteins (52) in the list of Top 100 proteins often identified in EVs are highlighted in bold (see Supplementary Table 3).

\begin{tabular}{|c|c|c|}
\hline Protein names & Gene names & $-\log 10(p-v a l u e)$ \\
\hline Band 4.1-like protein 2 & EPB41L2 & 9,852 \\
\hline Band 4.1-like protein 3 & $E P B 41 L 3$ & 8,468 \\
\hline Plasma membrane calcium-transporting ATPase 1 & ATP2B1 & 7,567 \\
\hline Phosphatidylinositol 4-kinase alpha & $P / 4 K A$ & 7,239 \\
\hline Tubulin-specific chaperone A & $T B C A$ & 6,731 \\
\hline Profilin-1 & PFN1 & 6,708 \\
\hline MARCKS-related protein & MARCKSL1 & 6,644 \\
\hline 4F2 cell-surface antigen heavy chain & SLC3A2 & 6,412 \\
\hline Cytoplasmic FMR1-interacting protein 1 & CYFIP1 & 6,209 \\
\hline Myelin protein zero-like protein 1 & MPZL1 & 6,167 \\
\hline $\mathrm{Na}(+) / \mathrm{H}(+)$ exchange regulatory cofactor NHE-RF1 & SLC9A3R1 & 6,151 \\
\hline Unconventional myosin-Ic & MYO1C & 5,986 \\
\hline Protein scribble homolog & SCRIB & 5,985 \\
\hline $\mathrm{N}(\mathrm{G}), \mathrm{N}(\mathrm{G})$-dimethylarginine dimethylaminohydrolase 1 & $D D A H 1$ & 5,900 \\
\hline 2,3-cyclic-nucleotide 3-phosphodiesterase & CNP & 5,696 \\
\hline Sodium/potassium-transporting ATPase subunit alpha-1 & ATP1A1 & 5,640 \\
\hline Lethal(2) giant larvae protein homolog 1 & LLGL1 & 5,620 \\
\hline FERM, RhoGEF and pleckstrin domain-containing protein 1 & FARP1 & 5,604 \\
\hline Integrin alpha-5 & ITGA5 & 5,589 \\
\hline Catenin delta-1 & CTNND1 & 5,556 \\
\hline Charged multivesicular body protein 6 & CHMP6 & 5,507 \\
\hline Basigin & $B S G$ & 5,349 \\
\hline PDZ domain-containing protein GIPC1 & GIPC1 & 5,304 \\
\hline Plastin-3 & PLS3 & 5,244 \\
\hline Peripheral plasma membrane protein CASK & CASK & 5,235 \\
\hline Trifunctional purine biosynthetic protein adenosine-3 & GART & 5,214 \\
\hline 14-3-3 protein epsilon & YWHAE & 5,213 \\
\hline Moesin & MSN & 5,169 \\
\hline Annexin A6 & ANXA6 & 5,150 \\
\hline
\end{tabular}


Myristoylated alanine-rich C-kinase substrate

Protein lin-7 homolog C

Guanine nucleotide-binding protein subunit alpha-13

Ubiquitin-40S ribosomal protein S27a

Immunoglobulin superfamily member 3

EGF-like repeat and discoidin I-like domain-containing protein 3

Sodium/potassium-transporting ATPase subunit beta-1

Unconventional myosin-lb

WD repeat-containing protein 6

Guanine nucleotide-binding protein G(i) subunit alpha-1

Inactive tyrosine-protein kinase 7

CD276 antigen

Sodium/potassium-transporting ATPase subunit beta-3

Catenin beta-1

Unconventional myosin-Id

Calcyclin-binding protein

Heat shock 70 kDa protein 4

Guanine nucleotide-binding protein $\mathrm{G}(\mathrm{I}) / \mathrm{G}(\mathrm{S}) / \mathrm{G}(\mathrm{O})$ subunit gamma-12

Afadin

Catenin alpha-1

Ras-related protein Rab-21

Kinesin-like protein KIF23

A-kinase anchor protein 12

Complement component $1 \mathrm{Q}$ subcomponent-binding protein

Peroxiredoxin-1

Neutral amino acid transporter B(0)

Fatty acid synthase

Pachytene checkpoint protein 2 homolog

Radixin

Phospholipid-transporting ATPase IG

Guanine nucleotide-binding protein $\mathrm{G}(\mathrm{s})$ subunit alpha isoforms

Nck-associated protein 1

C-1-tetrahydrofolate synthase

Disks large homolog 1

Glutathione S-transferase P

Creatine kinase B-type

Ectonucleotide pyrophosphatase/phosphodiesterase family member 1

Collagen alpha-1(XVIII) chain

Integrin alpha-1

14-3-3 protein beta/alpha

Cyclin-dependent kinase 1

Zinc transporter ZIP6

Microtubule-associated protein RP/EB family member 1

Large neutral amino acids transporter small subunit 1

Translation initiation factor elF-2B subunit alpha

Monocarboxylate transporter 1

14-3-3 protein theta

Ras GTPase-activating-like protein

\begin{tabular}{|c|c|}
\hline MARCKS & 5,105 \\
\hline LIN7C & 5,092 \\
\hline GNA13 & 5,054 \\
\hline$R P S 27 A ; U B B / C$ & 5,027 \\
\hline IGSF3 & 4,988 \\
\hline EDIL3 & 4,974 \\
\hline ATP1B1 & 4,960 \\
\hline MYO1B & 4,885 \\
\hline WDR6 & 4,826 \\
\hline GNAI1 & 4,814 \\
\hline PTK7 & 4,790 \\
\hline CD276 & 4,781 \\
\hline ATP1B3 & 4,738 \\
\hline CTNNB1 & 4,699 \\
\hline MYO1D & 4,582 \\
\hline CACYBP & 4,579 \\
\hline HSPA4 & 4,550 \\
\hline GNG12 & 4,474 \\
\hline MLLT4 & 4,449 \\
\hline CTNNA1 & 4,449 \\
\hline RAB21 & 4,444 \\
\hline KIF23 & 4,376 \\
\hline AKAP12 & 4,302 \\
\hline$C 1 Q B P$ & 4,296 \\
\hline PRDX1 & 4,263 \\
\hline SLC1A5 & 4,221 \\
\hline FASN & 4,138 \\
\hline TRIP13 & 4,113 \\
\hline$R D X$ & 4,089 \\
\hline ATP11C & 4,077 \\
\hline GNAS & 4,055 \\
\hline NCKAP1 & 4,054 \\
\hline MTHFD1 & 4,019 \\
\hline$D L G 1$ & 4,010 \\
\hline GSTP1 & 4,008 \\
\hline$C K B$ & 4,007 \\
\hline ENPP1 & 3,995 \\
\hline COL18A1 & 3,995 \\
\hline ITGA1 & 3,987 \\
\hline YWHAB & 3,953 \\
\hline$C D K 1$ & 3,950 \\
\hline SLC39A6 & 3,947 \\
\hline MAPRE1 & 3,914 \\
\hline SLC7A5 & 3,865 \\
\hline EIF2B1 & 3,790 \\
\hline SLC16A1 & 3,788 \\
\hline$Y W H A Q$ & 3,784 \\
\hline IQGAP1 & 3,751 \\
\hline
\end{tabular}


14-3-3 protein eta

\begin{tabular}{|c|c|}
\hline YWHAH & 3,743 \\
\hline PRDX6 & 3,729 \\
\hline CYFIP2 & 3,687 \\
\hline KIF5B & 3,680 \\
\hline NCSTN & 3,677 \\
\hline GNAI3 & 3,644 \\
\hline ANXA2 & 3,628 \\
\hline DCTPP1 & 3,580 \\
\hline ITGB1 & 3,579 \\
\hline DNAJC5 & 3,575 \\
\hline FHL1 & 3,567 \\
\hline ACTG1 & 3,555 \\
\hline HSP90B1 & 3,554 \\
\hline BOLA2 & 3,550 \\
\hline$P N P$ & 3,538 \\
\hline HSPG2 & 3,487 \\
\hline PTPRF & 3,433 \\
\hline PHGDH & 3,433 \\
\hline UNC45A & 3,432 \\
\hline IRS4 & 3,430 \\
\hline GNAI2 & 3,417 \\
\hline$B A S P 1$ & 3,383 \\
\hline$E Z R$ & 3,375 \\
\hline CFL2 & 3,371 \\
\hline HSPE1 & 3,363 \\
\hline NDRG1 & 3,352 \\
\hline CXADR & 3,347 \\
\hline$P C B P 2$ & 3,321 \\
\hline UCHL1 & 3,321 \\
\hline GNA11 & 3,303 \\
\hline HSP90AB1 & 3,294 \\
\hline HSPH1 & 3,259 \\
\hline RAB10 & 3,255 \\
\hline RHEB & 3,252 \\
\hline$R P 2$ & 3,245 \\
\hline SLC29A1 & 3,219 \\
\hline UNC13D & 3,212 \\
\hline YWHAZ & 3,209 \\
\hline$P C B P 1$ & 3,208 \\
\hline EPHA2 & 3,206 \\
\hline PLXNA1 & 3,205 \\
\hline HSPA1B;HSPA1A & 3,197 \\
\hline HPRT1 & 3,188 \\
\hline PDCD5 & 3,183 \\
\hline TCP1 & 3,167 \\
\hline ANXA1 & 3,162 \\
\hline$P H B$ & 3,152 \\
\hline CLIC4 & 3,144 \\
\hline
\end{tabular}

Peroxiredoxin-6

Cytoplasmic FMR1-interacting protein 2

\begin{tabular}{l}
\hline Kinesin-1 heavy chain \\
\hline Nicastrin
\end{tabular}

Guanine nucleotide-binding protein $\mathrm{G}(\mathrm{k})$ subunit alpha

\begin{tabular}{l|l}
\hline Annexin A2 & A \\
\hline dCTP pyrophosphatase 1 & \\
\hline Integrin beta-1 &
\end{tabular}

DnaJ homolog subfamily C member 5

Four and a half LIM domains protein 1

Actin, cytoplasmic 2

Endoplasmin

BolA-like protein 2

Purine nucleoside phosphorylase

Basement membrane-specific heparan sulfate proteoglycan core protein

Receptor-type tyrosine-protein phosphatase $\mathrm{F}$

D-3-phosphoglycerate dehydrogenase

Protein unc-45 homolog $\mathrm{A}$

Insulin receptor substrate 4

Guanine nucleotide-binding protein $\mathrm{G}(\mathrm{i})$ subunit alpha-2

Brain acid soluble protein 1

Ezrin

Cofilin-2

$10 \mathrm{kDa}$ heat shock protein, mitochondrial

Protein NDRG1

Coxsackievirus and adenovirus receptor

Poly(rC)-binding protein 2

Ubiquitin carboxyl-terminal hydrolase isozyme L1

Guanine nucleotide-binding protein subunit alpha-11

Heat shock protein HSP 90-beta

Heat shock protein $105 \mathrm{kDa}$

Ras-related protein Rab-10

GTP-binding protein Rheb

Protein XRP2

Equilibrative nucleoside transporter 1

Protein unc-13 homolog $D$

14-3-3 protein zeta/delta

Poly(rC)-binding protein 1

Ephrin type-A receptor 2

Plexin-A1

Heat shock $70 \mathrm{kDa}$ protein 1B; Heat shock $70 \mathrm{kDa}$ protein $1 \mathrm{~A}$

Hypoxanthine-guanine phosphoribosyltransferase

Programmed cell death protein 5

T-complex protein 1 subunit alpha

\section{Annexin A1}

Prohibitin

Chloride intracellular channel protein 4 
Leucine-rich repeat-containing protein 57

Neurogenic locus notch homolog protein 2

Ras-related protein Rab-7a

Junctional adhesion molecule C

Nidogen-2

Small VCP/p97-interacting protein

MOB kinase activator 1A;MOB kinase activator 1B

Inorganic pyrophosphatase

Ras-related protein Rab-23

Zinc finger $\mathrm{CCCH}$-type antiviral protein 1

HLA class I histocompatibility antigen, A-2 alpha chain

Glypican-4;Secreted glypican-4

Phosphoribosylformylglycinamidine synthase

L-lactate dehydrogenase $A$ chain

T-complex protein 1 subunit epsilon

Single-stranded DNA-binding protein, mitochondrial

HLA class I histocompatibility antigen, B-7 alpha chain

Syntaxin-4

Integrin alpha-V

Synaptosomal-associated protein 23

Fascin

Peroxiredoxin-2

14-3-3 protein gamma

Guanine nucleotide-binding protein $\mathrm{G}(\mathrm{I}) / \mathrm{G}(\mathrm{S}) / \mathrm{G}(\mathrm{T})$ subunit beta-1

Rho GDP-dissociation inhibitor 1

Copine-3

Triosephosphate isomerase

ADP-sugar pyrophosphatase

Solute carrier family 12 member 2

Elongation factor 1-alpha 1;Putative elongation factor 1-alpha-like 3

I

\begin{tabular}{l|l} 
LRRC57 & \\
\hline NOTCH2 & \\
\hline RAB7A &
\end{tabular}

3,142

JAM3

3,137

NID2

3,108

SVIP

3,103

\begin{tabular}{l|l} 
MOB1A;MOB1B & 3,090 \\
\hline
\end{tabular}

\begin{tabular}{|l|r}
\hline$P P A 1$ & 3,088 \\
\hline
\end{tabular}

\begin{tabular}{|l|l}
\hline RAB23 & 3,071 \\
\hline
\end{tabular}

\begin{tabular}{|l|l}
\hline ZC3HAV1 & 3,039 \\
\hline
\end{tabular}

\begin{tabular}{l|l}
\hline HLA-A & 3,034 \\
\hline
\end{tabular}

\begin{tabular}{l|l}
\hline GPC4 & 3,033 \\
\hline
\end{tabular}

\begin{tabular}{|r|r}
\hline PFAS & 2,977 \\
\hline
\end{tabular}

\begin{tabular}{|r|r}
\hline LDHA & 2,965 \\
\hline
\end{tabular}

\begin{tabular}{l|r} 
CCT5 & 2,944 \\
\hline
\end{tabular}

\begin{tabular}{|l|r}
\hline$S S B P 1$ & 2,938 \\
\hline
\end{tabular}

\begin{tabular}{l|l}
\hline$H L A-B$ & 2,909 \\
\hline
\end{tabular}

\begin{tabular}{l|r}
\hline$S T X 4$ & 2,907 \\
\hline
\end{tabular}

\begin{tabular}{|r|r}
\hline ITGAV & 2,885 \\
\hline
\end{tabular}

\begin{tabular}{|r|r}
\hline SNAP23 & 2,884 \\
\hline
\end{tabular}

\begin{tabular}{|r|r}
\hline FSCN1 & 2,883 \\
\hline
\end{tabular}

\begin{tabular}{|l|r}
\hline PRDX2 & 2,876 \\
\hline
\end{tabular}

\begin{tabular}{|l|l}
\hline YWHAG & $\mathbf{2 , 8 7 4}$ \\
\hline
\end{tabular}

\begin{tabular}{l|r}
\hline GNB1 & 2,860 \\
\hline
\end{tabular}

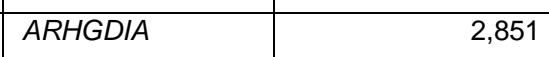

\begin{tabular}{|r|r}
\hline CPNE3 & 2,845 \\
\hline
\end{tabular}

\begin{tabular}{l|l} 
TPI1 & $\mathbf{2 , 8 4 2}$ \\
\hline
\end{tabular}

\begin{tabular}{l|r} 
NUDT5 & $\mathbf{2 , 8 4 2}$ \\
\hline SLC12A2 & 2,839 \\
\hline EEF1A1;EEF1A1 & 2,802 \\
\hline 5 & $\mathbf{2 , 7 6 1}$ \\
\hline
\end{tabular}

\begin{tabular}{|c|c|c|}
\hline Synaptic vesicle membrane protein VAT-1 homolog & VAT1 & 2,731 \\
\hline T-complex protein 1 subunit eta & CCT7 & 2,729 \\
\hline T-complex protein 1 subunit zeta & CCT6A & 2,728 \\
\hline Serine/threonine-protein kinase MRCK beta & $C D C 42 B P B$ & 2,699 \\
\hline Multifunctional protein ADE2 & PAICS & 2,688 \\
\hline Protein FAM49B & FAM49B & 2,686 \\
\hline Ras-related protein Rab-5C & RAB5C & 2,680 \\
\hline Receptor-type tyrosine-protein phosphatase gamma & PTPRG & 2,677 \\
\hline Myosin light polypeptide 6 & MYL6 & 2,673 \\
\hline Destrin & DSTN & 2,673 \\
\hline Fibronectin & FN1 & 2,672 \\
\hline Gap junction alpha-1 protein & GJA1 & 2,665 \\
\hline LIM and SH3 domain protein 1 & LASP1 & 2,647 \\
\hline Aspartate aminotransferase, cytoplasmic & GOT1 & 2,643 \\
\hline CTP synthase 1 & CTPS1 & 2,640 \\
\hline Guanine nucleotide-binding protein $G(I) / G(S) / G(T)$ subunit beta-2 & GNB2 & 2,631 \\
\hline Collagen alpha-1(IV) chain & COL4A1 & 2,630 \\
\hline
\end{tabular}


Ras-related protein Rab-8B

Elongation factor 1-delta

T-complex protein 1 subunit delta

Serine/threonine-protein kinase 26

$60 \mathrm{kDa}$ heat shock protein, mitochondrial

Integrin alpha-2

Disks large homolog 3

Calcineurin B homologous protein 1

Ras-related protein Rab-35

Protein disulfide-isomerase A6

Ras-related protein Ral-A

Elongation factor 1-beta

Nuclear migration protein nudC

Mitogen-activated protein kinase kinase kinase kinase 4

Choline transporter-like protein 2

Insulin-like growth factor 1 receptor

\section{Glyceraldehyde-3-phosphate dehydrogenase}

Ras-related protein R-Ras2

Protein deglycase DJ-1

Coatomer subunit alpha;Xenin;Proxenin

Importin subunit beta-1

T-complex protein 1 subunit beta

Ras GTPase-activating protein 3

Importin-4

Presenilin-1

2-deoxynucleoside 5-phosphate N-hydrolase 1

Prohibitin-2

Heat shock protein HSP 90-alpha

Elongation factor Tu, mitochondrial

Glycine-tRNA ligase

GTPase HRas

Pyruvate kinase PKM

\begin{tabular}{|c|c|c|}
\hline & & \\
\hline Guanine nucleotide-binding protein $\mathrm{G}(\mathrm{o})$ subunit alpha & GNAO1 & 2,351 \\
\hline Rac GTPase-activating protein 1 & RACGAP1 & 2,343 \\
\hline ATP-dependent RNA helicase DDX3X/ DDX3Y & $D D X 3 X ; D D X 3 Y$ & 2,341 \\
\hline Zinc transporter ZIP14 & SLC39A14 & 2,336 \\
\hline Sorting nexin-5 & SNX5 & 2,330 \\
\hline Ubiquitin-conjugating enzyme E2 K & UBE2K & 2,325 \\
\hline Thioredoxin domain-containing protein 17 & TXNDC17 & 2,317 \\
\hline Cofilin-1 & CFL1 & $2, \mathbf{3 1 3}$ \\
\hline Heterogeneous nuclear ribonucleoprotein A3 & HNRNPA3 & 2,310 \\
\hline DNA topoisomerase 2-alpha & TOP2A & 2,308 \\
\hline Calcium-binding protein 39 & CAB39 & 2,290 \\
\hline Collagen alpha-2(IV) chain;Canstatin & COL4A2 & 2,285 \\
\hline Lactoylglutathione lyase & GLO1 & 2,281 \\
\hline Rho-associated protein kinase 1 & ROCK1 & 2,271 \\
\hline Centrosomal protein of $55 \mathrm{kDa}$ & CEP55 & 2,268 \\
\hline Nucleoside diphosphate kinase B;Putative nucleoside diphosphate kinase & NME2;NME2P1 & 2,255 \\
\hline
\end{tabular}


Ras-related C3 botulinum toxin substrate 1

\begin{tabular}{|c|c|c|}
\hline Ras-related C3 botulinum toxin substrate 1 & RAC1 & 2,246 \\
\hline Neuropilin-1 & NRP1 & 2,227 \\
\hline $\begin{array}{l}\text { Phosphatidylethanolamine-binding protein 1; Hippocampal cholinergic } \\
\text { neurostimulating peptide }\end{array}$ & PEBP1 & 2,217 \\
\hline Integrin alpha-6 & ITGA6 & 2,203 \\
\hline Protein kinase $C$ beta type & $P R K C B$ & 2,196 \\
\hline Fatty acid-binding protein, epidermal & FABP5 & 2,190 \\
\hline T-complex protein 1 subunit theta & CCT8 & 2,180 \\
\hline Endophilin-B1 & SH3GLB1 & 2,168 \\
\hline AP-2 complex subunit beta & $A P 2 B 1$ & 2,147 \\
\hline Melanoma-associated antigen D2 & MAGED2 & 2,127 \\
\hline Stathmin & STMN1 & 2,123 \\
\hline Annexin A3 & ANXA3 & 2,100 \\
\hline Fructose-bisphosphate aldolase A & $A L D O A$ & 2,100 \\
\hline Heat shock cognate $71 \mathrm{kDa}$ protein & HSPA8 & 2,096 \\
\hline Alpha-enolase & ENO1 & 2,093 \\
\hline Guanine nucleotide-binding protein $\mathrm{G}(\mathrm{q})$ subunit alpha & GNAQ & 2,090 \\
\hline Phosphatidylinositol transfer protein beta isoform & PITPNB & 2,089 \\
\hline Junction plakoglobin & JUP & 2,075 \\
\hline Filamin-B & FLNB & 2,072 \\
\hline Golgin subfamily A member 7 & GOLGA7 & 2,071 \\
\hline Programmed cell death protein 10 & PDCD10 & 2,062 \\
\hline Neuroblast differentiation-associated protein AHNAK & AHNAK & 2,045 \\
\hline Puromycin-sensitive aminopeptidase & NPEPPS & 2,044 \\
\hline Lysyl oxidase homolog 2 & LOXL2 & 2,043 \\
\hline Transgelin-2 & TAGLN2 & 2,032 \\
\hline Anosmin-1 & KAL1 & 2,029 \\
\hline Cyclin-Y & CCNY & 2,016 \\
\hline Laminin subunit alpha-5 & LAMA5 & 2,015 \\
\hline Nucleoside diphosphate kinase A & NME1 & 2,011 \\
\hline Rho-related GTP-binding protein RhoG & $R H O G$ & 2,008 \\
\hline Serine/threonine-protein kinase TAO1 & TAOK1 & 1,998 \\
\hline Proliferating cell nuclear antigen & $P C N A$ & 1,991 \\
\hline BTB/POZ domain-containing protein KCTD12 & KCTD12 & 1,977 \\
\hline Staphylococcal nuclease domain-containing protein 1 & SND1 & 1,963 \\
\hline CD59 glycoprotein & CD59 & 1,961 \\
\hline Junctional adhesion molecule A & $F 11 R$ & 1,959 \\
\hline Annexin A5 & ANXA5 & 1,957 \\
\hline Protein NDNF & $N D N F$ & 1,956 \\
\hline NADH-cytochrome b5 reductase 3 & CYB5R3 & 1,953 \\
\hline Ras-related protein Rab-13 & RAB13 & 1,943 \\
\hline Occludin & OCLN & 1,938 \\
\hline Glypican-6;Secreted glypican-6 & GPC6 & 1,933 \\
\hline CAD protein & $C A D$ & 1,909 \\
\hline Lysosome-associated membrane glycoprotein 1 & LAMP1 & 1,899 \\
\hline Protein-L-isoaspartate(D-aspartate) O-methyltransferase & PCMT1 & 1,898 \\
\hline Activator of $90 \mathrm{kDa}$ heat shock protein ATPase homolog 1 & AHSA1 & 1,886 \\
\hline Fermitin family homolog 2 & FERMT2 & 1,875 \\
\hline
\end{tabular}


Band 4.1-like protein 5

\begin{tabular}{l}
\hline STE20-like serine/threonine-protein kinase \\
\hline Stress-70 protein, mitochondrial
\end{tabular}

DnaJ homolog subfamily B member 1

Malate dehydrogenase, mitochondrial

Protein EFR3 homolog A

\begin{tabular}{l|l}
\hline ATP synthase subunit alpha, mitochondrial & $A$ \\
\hline Agrin & $A$
\end{tabular}

\begin{tabular}{l|l}
\hline Voltage-dependent anion-selective channel protein 2 & \\
\hline Hsc70-interacting protein & \\
\hline F-actin-capping protein subunit beta & \\
\hline POTE ankyrin domain family member E / F & $P$
\end{tabular}

\begin{tabular}{l|l}
\hline Gamma-aminobutyric acid receptor-associated protein-like 2 & $G$ \\
\hline Teneurin-3
\end{tabular}

\begin{tabular}{l|l}
\hline Histone $\mathrm{H} 1.0$ & $H$
\end{tabular}

Ubiquitin-like modifier-activating enzyme 1

Eukaryotic translation initiation factor 2 subunit 1

Desmoglein-2

\section{Elongation factor 2}

GTPase NRas

ATP synthase subunit beta, mitochondrial

Aspartate--tRNA ligase, cytoplasmic

E3 ubiquitin-protein ligase CHIP

Developmentally-regulated GTP-binding protein 1

Protein-glutamine gamma-glutamyltransferase $\mathrm{E}$

Tyrosine-protein kinase Lyn

\section{Clathrin heavy chain 1}

Epidermal growth factor receptor

Serine/threonine-protein phosphatase PP1-alpha catalytic subunit

Thioredoxin-dependent peroxide reductase, mitochondrial

Malate dehydrogenase, cytoplasmic

Syntaxin-7

Inosine-5-monophosphate dehydrogenase 2

Ran-specific GTPase-activating protein

$78 \mathrm{kDa}$ glucose-regulated protein

Cell division control protein 42 homolog

DnaJ homolog subfamily A member 2

\section{L-lactate dehydrogenase $B$ chain}

\begin{tabular}{|c|c|c|}
\hline ADP/ATP translocase 2 & SLC25A5 & 1,585 \\
\hline Profilin-2 & PFN2 & 1,580 \\
\hline Phosphatidylinositol 5-phosphate 4-kinase type-2 alpha & PIP4K2A & 1,580 \\
\hline Alanine--tRNA ligase, cytoplasmic & $A A R S$ & 1,560 \\
\hline D-dopachrome decarboxylase;D-dopachrome decarboxylase-like protein & $D D T ; D D T L$ & 1,555 \\
\hline Protein 4.1 & EPB41 & 1,553 \\
\hline Histone $\mathrm{H} 2 \mathrm{~B}$ type $1-\mathrm{C} / \mathrm{E} / \mathrm{F} / \mathrm{G} / \mathrm{I}$ & HIST1H2BC & 1,537 \\
\hline Long-chain-fatty-acid--CoA ligase 4 & ACSL4 & 1,535 \\
\hline Rab GDP dissociation inhibitor beta & GDI2 & 1,524 \\
\hline Phosphoribosyl pyrophosphate synthase-associated protein 2 & PRPSAP2 & 1,523 \\
\hline
\end{tabular}


Ras-related protein Rap-2b

Choline transporter-like protein 1

NudC domain-containing protein 2

Plasma membrane calcium-transporting ATPase 4

Ras-related protein Rab-8A

Axin interactor, dorsalization-associated protein

High affinity cationic amino acid transporter 1

$\mathrm{N}$-alpha-acetyltransferase 15 , NatA auxiliary subunit

Histone $\mathrm{H} 1.4$

Translational activator GCN1

Caspase-3;Caspase-3 subunit p17;Caspase-3 subunit p12

Proliferation-associated protein $2 \mathrm{G} 4$

Histone H2A type 1C; Histone H2A type 3; Histone H2A type 1-B/E

Eukaryotic peptide chain release factor subunit 1

Ephrin type-A receptor 7

AP-2 complex subunit mu

Raftlin

ADP-ribosylation factor 6

Macrophage migration inhibitory factor

$\mathrm{Na}(+) / \mathrm{H}(+)$ exchange regulatory cofactor NHE-RF2

Unconventional myosin- $\mathrm{X}$

Aldose reductase

Ubiquitin-conjugating enzyme E2 L3

Synaptobrevin homolog YKT6

Transaldolase

Ras-related protein Ral-B

Barrier-to-autointegration factor

Nidogen-1

Protein kinase $\mathrm{C}$ and casein kinase substrate in neurons protein 3

V-type proton ATPase $116 \mathrm{kDa}$ subunit a isoform 1

Tubulin beta chain

Protein S100-A10

Ribose-phosphate pyrophosphokinase 1

Adenine phosphoribosyltransferase

Guanine nucleotide-binding protein $\mathrm{G}(\mathrm{I}) / \mathrm{G}(\mathrm{S}) / \mathrm{G}(\mathrm{O})$ subunit gamma-10

von Willebrand factor A domain-containing protein 1

Arginine--tRNA ligase, cytoplasmic

Pre-mRNA-processing-splicing factor 8

Polyadenylate-binding protein 1;Polyadenylate-binding protein 3

Prefoldin subunit 2

Syntaxin-3

Vesicle-associated membrane protein 3

Astrocytic phosphoprotein PEA-15

Cysteine and histidine-rich domain-containing protein 1

Histone H2A type 2-C;Histone H2A type 2-A

\begin{tabular}{|c|c|}
\hline$R A P 2 B$ & 1,517 \\
\hline SLC44A1 & 1,516 \\
\hline NUDCD2 & 1,513 \\
\hline ATP2B4 & 1,504 \\
\hline$R A B 8 A$ & 1,503 \\
\hline$A I D A$ & 1,492 \\
\hline SLC7A1 & 1,492 \\
\hline NAA15 & 1,488 \\
\hline HIST1H1E & 1,469 \\
\hline GCN1L1 & 1,462 \\
\hline CASP3 & 1,458 \\
\hline PA2G4 & 1,458 \\
\hline $\begin{array}{l}\text { HIST1H2AC;HIST } \\
3 H 2 A ; H I S T 1 H 2 A B\end{array}$ & 1,454 \\
\hline ETF1 & 1,436 \\
\hline EPHA7 & 1,434 \\
\hline$A P 2 M 1$ & 1,407 \\
\hline RFTN1 & 1,400 \\
\hline ARF6 & 1,400 \\
\hline MIF & 1,392 \\
\hline SLC9A3R2 & 1,391 \\
\hline MYO10 & 1,386 \\
\hline$A K R 1 B 1$ & 1,384 \\
\hline UBE2L3 & 1,381 \\
\hline YKT6 & 1,379 \\
\hline TALDO1 & 1,377 \\
\hline$R A L B$ & 1,377 \\
\hline BANF1 & 1,373 \\
\hline NID1 & 1,361 \\
\hline PACSIN3 & 1,360 \\
\hline$\overline{A T P 6 V 0 A 1}$ & 1,345 \\
\hline TUBB & 1,343 \\
\hline S100A10 & 1,340 \\
\hline$\overline{P R P S 1}$ & 1,337 \\
\hline$A P R T$ & 1,333 \\
\hline GNG10 & 1,333 \\
\hline VWA1 & 1,328 \\
\hline$R A R S$ & 1,308 \\
\hline PRPF8 & 1,298 \\
\hline$P A B P C 1 ; P A B P C 3$ & 1,286 \\
\hline PFDN2 & 1,286 \\
\hline STX3 & 1,282 \\
\hline$\overline{V A M P 3}$ & 1,276 \\
\hline PEA15 & 1,276 \\
\hline CHORDC1 & 1,275 \\
\hline $\begin{array}{l}\text { HIST2H2AC;HIST } \\
2 H 2 A A 3\end{array}$ & 1,251 \\
\hline VIM & 1,249 \\
\hline
\end{tabular}

Vimentin 
ATP-binding cassette sub-family E member 1

\begin{tabular}{|c|c|c|}
\hline Filamin-C & FLNC & 1,234 \\
\hline Syndecan-2 & SDC2 & 1,224 \\
\hline Disco-interacting protein 2 homolog $B$ & DIP2B & 1,211 \\
\hline Calnexin & CANX & 1,201 \\
\hline Heterogeneous nuclear ribonucleoprotein U & HNRNPU & 1,192 \\
\hline Probable ATP-dependent RNA helicase DDX5 & $D D \times 5$ & 1,176 \\
\hline DNA damage-binding protein 1 & $D D B 1$ & 1,170 \\
\hline $40 S$ ribosomal protein S23 & RPS23 & 1,166 \\
\hline Protein disulfide-isomerase A4 & PDIA4 & 1,165 \\
\hline Poly [ADP-ribose] polymerase 1 & PARP1 & 1,114 \\
\hline
\end{tabular}

Supplementary Table 3. Table of the Top 100 proteins often identified in EVs (source: vesiclepedia, http://microvesicles.org/extracellular vesicle markers ).

\begin{tabular}{|c|c|}
\hline Gene & Number of times identified \\
\hline PDCD6IP & 399 \\
\hline GAPDH & 377 \\
\hline HSPA8 & 363 \\
\hline ACTB & 350 \\
\hline ANXA2 & 337 \\
\hline CD9 & 328 \\
\hline$P K M$ & 327 \\
\hline HSP90AA1 & 327 \\
\hline ENO1 & 327 \\
\hline ANXA5 & 313 \\
\hline HSP90AB1 & 306 \\
\hline$C D 63$ & 306 \\
\hline YWHAZ & 301 \\
\hline YWHAE & 300 \\
\hline EEF1A1 & 295 \\
\hline PGK1 & 291 \\
\hline CLTC & 283 \\
\hline PPIA & 278 \\
\hline$S D C B P$ & 277 \\
\hline ALDOA & 275 \\
\hline$E E F 2$ & 274 \\
\hline$A L B$ & 274 \\
\hline TPI1 & 270 \\
\hline$V C P$ & 269 \\
\hline CFL1 & 268 \\
\hline$M S N$ & 266 \\
\hline ATP1A1 & 266 \\
\hline$P R D X 1$ & 263 \\
\hline MYH9 & 262 \\
\hline$E Z R$ & 262 \\
\hline$C D 81$ & 262 \\
\hline ANXA6 & 260 \\
\hline FLOT1 & 259 \\
\hline YWHAB & 258 \\
\hline
\end{tabular}




\begin{tabular}{|c|c|}
\hline$L D H B$ & 258 \\
\hline SLC3A2 & 257 \\
\hline GNB1 & 257 \\
\hline PFN1 & 256 \\
\hline TSG101 & 255 \\
\hline YWHAQ & 254 \\
\hline GNAI2 & 252 \\
\hline CLIC1 & 251 \\
\hline$A N X A 1$ & 251 \\
\hline ITGB1 & 250 \\
\hline LDHA & 249 \\
\hline FASN & 248 \\
\hline$C D C 42$ & 248 \\
\hline$R A P 1 B$ & 242 \\
\hline CCT2 & 242 \\
\hline YWHAG & 240 \\
\hline GNB2 & 240 \\
\hline ACTN4 & 240 \\
\hline$R A B 5 C$ & 239 \\
\hline C3 & 239 \\
\hline$R A B 10$ & 236 \\
\hline HIST1H4A & 234 \\
\hline KRT1 & 233 \\
\hline FN1 & 233 \\
\hline$A H C Y$ & 233 \\
\hline$A 2 M$ & 232 \\
\hline BSG & 230 \\
\hline$A C T N 1$ & 229 \\
\hline ANXA7 & 228 \\
\hline$A C L Y$ & 228 \\
\hline HIST1H4B & 227 \\
\hline GDI2 & 227 \\
\hline FLNA & 227 \\
\hline UBA1 & 226 \\
\hline GNAS & 226 \\
\hline GSN & 225 \\
\hline CCT4 & 225 \\
\hline$R A N$ & 222 \\
\hline PRDX2 & 222 \\
\hline RHOA & 220 \\
\hline CCT3 & 220 \\
\hline RAC1 & 219 \\
\hline LGALS3BP & 219 \\
\hline TCP1 & 218 \\
\hline KRT10 & 218 \\
\hline CAP1 & 218 \\
\hline RAB7A & 217 \\
\hline TUBB4B & 216 \\
\hline HSPA5 & 215 \\
\hline IQGAP1 & 214 \\
\hline GPI & 214 \\
\hline
\end{tabular}




\begin{tabular}{c|c} 
RALA & 213 \\
\hline KPNB1 & 212 \\
\hline HIST1H4I & 212 \\
\hline TFRC & 211 \\
\hline EIF4A1 & 211 \\
\hline HIST4H4 & 210 \\
\hline CCT8 & 210 \\
\hline TLN1 & 209 \\
\hline HIST1H4K & 209 \\
\hline HIST1H4H & 209 \\
\hline CCT6A & 209 \\
\hline ANXA11 & 209 \\
\hline HIST1H4J & 208 \\
\hline HIST1H4F & 208 \\
\hline HIST1H4D & 208 \\
\hline
\end{tabular}




\subsection{Publication II}

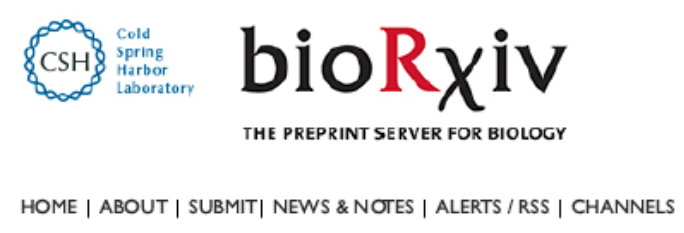

Advanced Search

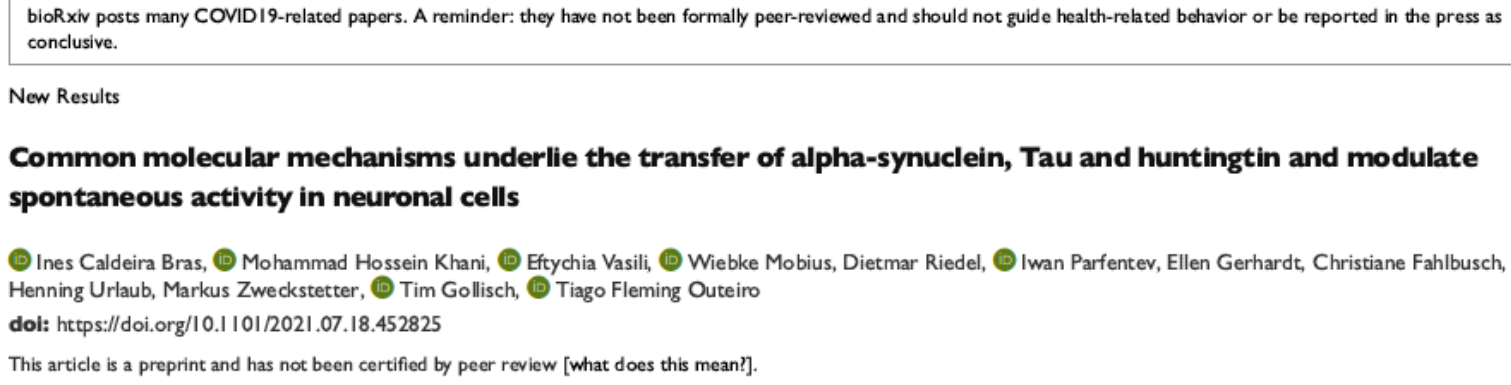

Ines Caldeira Bras, Mohammad Hossein Khani, Eftychia Vasili, Wiebke Mobius, Dietmar Riedel, Iwan Parfentev, Ellen Gerhardt, Christiane Fahlbusch, Henning Urlaub, Markus Zweckstetter, Tim Gollisch, Tiago Fleming Outeiro doi: https://doi.org/10.1 101/2021.07.18.452825

This article is a preprint and has not been certified by peer review [what does this mean?].

Link: https://www.biorxiv.org/content/10.1101/2021.07.18.452825v1

\section{Author contributions}

Inês Caldeira Brás and Tiago Fleming Outeiro conceived the study. Inês Caldeira Brás performed all the cell culture, molecular biology and imaging experiments. Inês Caldeira Brás and Mohammad Hossein Khani stablished the spike sorting framework, performed multielectrode array experiments and data analysis. Eftychia Vasili prepared the microglia samples for quantitative-PCR, imaging and recombinant alpha-synuclein. Wiebke Möbius performed the immuno-electron microscopy experiments in the stable cell lines. Dietmar Riedel performed the electron microscopy experiments with the extracellular vesicles. Ellen Gerhardt performed the cloning and prepared the lentiviral vectors. Christiane Fahlbusch prepared the lentiviral vectors. Iwan Parfentev and Henning Urlaub performed the mass spectrometry experiments. Markus Zweckstetter provided the recombinant full-length 2N4R Tau. Tim Gollisch provided methodology and resources for the multi-electrode array experiments. Inês Caldeira Brás analysed and interpreted the data. Inês Caldeira Brás generated the graphs and figures. Inês Caldeira Brás and Tiago Fleming Outeiro wrote the manuscript. Tiago Fleming Outeiro supervised the work. 


\section{Graphical abstract}
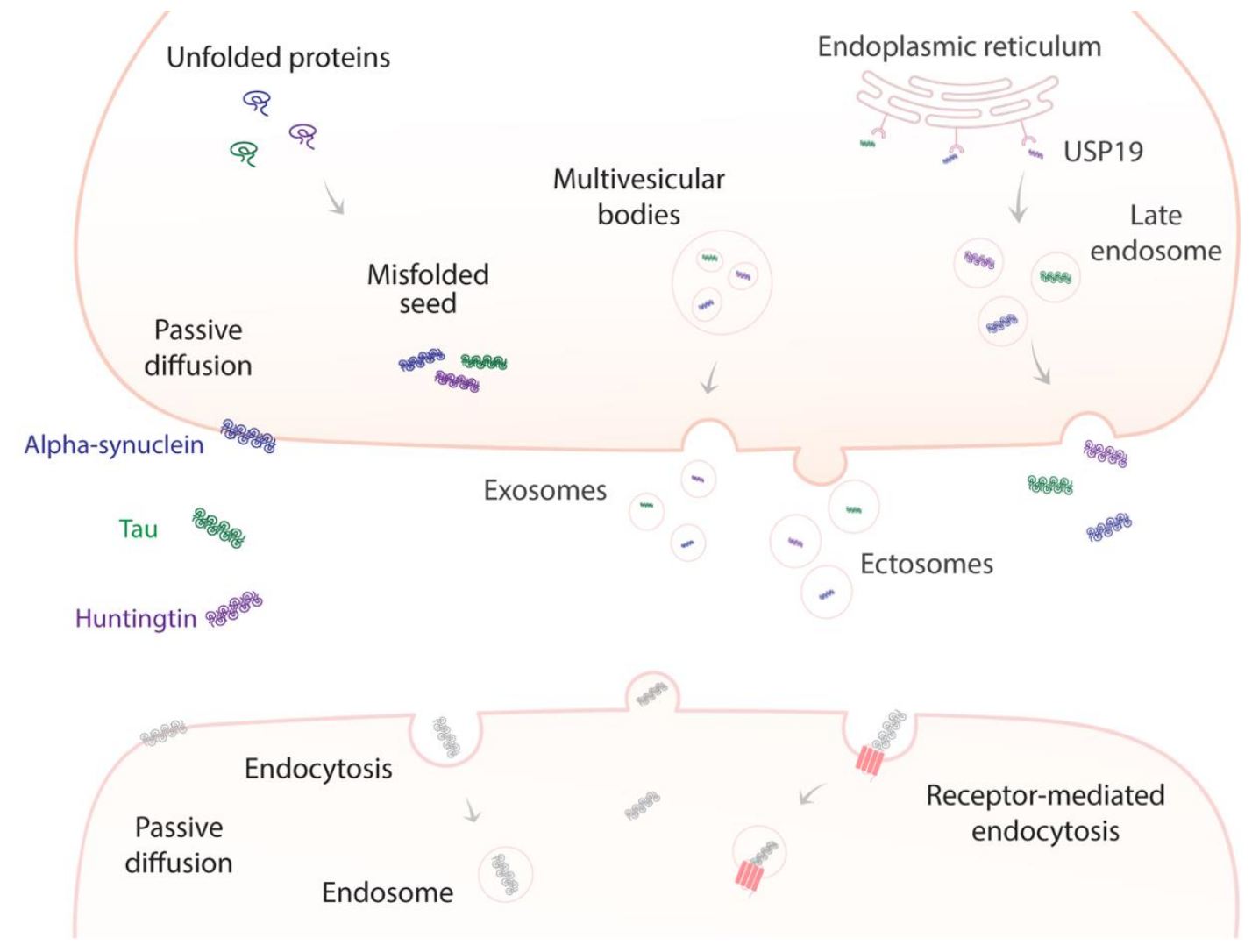


\section{Common molecular mechanisms underlie the transfer of alpha-synuclein, Tau and huntingtin and modulate spontaneous activity in neuronal cells}

Inês Caldeira Brás ${ }^{1}$, Mohammad Hossein Khani², Eftychia Vasili ${ }^{1}$, Wiebke Möbius ${ }^{3,4}$, Dietmar Riedel ${ }^{5}$, Iwan Parfentev ${ }^{6}$, Ellen Gerhardt ${ }^{1}$, Christiane Fahlbusch ${ }^{1}$, Henning Urlaub $^{6,7}$, Markus Zweckstetter $8,9,10$, Tim Gollisch², Tiago Fleming Outeiro ${ }^{1,11,12,13^{*}}$

1Department of Experimental Neurodegeneration, Center for Biostructural Imaging of Neurodegeneration, University Medical Center Göttingen, 37073 Göttingen, Germany.

2Department of Ophthalmology, University Medical Center Göttingen, 37073 Göttingen, Germany.

${ }^{3}$ Department of Neurogenetics, Max Planck Institute for Experimental Medicine, 37075 Göttingen, Germany.

${ }^{4}$ Electron Microscopy Core Unit, Max Planck Institute for Experimental Medicine, 37075 Göttingen, Germany.

${ }^{5}$ Laboratory of Electron Microscopy, Max Planck Institute for Biophysical Chemistry, 37075 Göttingen, Germany.

${ }^{6}$ Research Group Bioanalytical Mass Spectrometry, Max-Planck-Institute for Biophysical Chemistry, 37077 Göttingen, Germany.

${ }^{7}$ Bioanalytics, Institute of Clinical Chemistry, University Medical Center Göttingen, 37075 Göttingen, Germany.

${ }^{8}$ German Center for Neurodegenerative Diseases (DZNE), 37075 Göttingen, Germany.

9Department for NMR-based Structural Biology, Max Planck Institute for Biophysical Chemistry, 37077 Göttingen, Germany.

${ }^{10}$ Department of Neurology, University Medical Center Göttingen, 37073 Göttingen, Germany.

${ }^{11}$ Max Planck Institute for Experimental Medicine, 37075 Göttingen, Germany.

${ }^{12}$ Translational and Clinical Research Institute, Faculty of Medical Sciences, Newcastle University, NE2 4HH, United Kingdom.

${ }^{13}$ Scientific employee with an honorary contract at German Center for Neurodegenerative Diseases (DZNE), 37075 Göttingen, Germany.

\section{*Correspondence to:}

Prof. Dr. Tiago Fleming Outeiro

Department of Experimental Neurodegeneration, University Medical Center Göttingen, 37073

Göttingen, Germany

E-mail: touteir@gwdg.de

Office Phone: +49 (0) 5513913544

Fax: +49 (0) 5513922693 


\section{Abstract}

The misfolding and accumulation of disease-related proteins are common hallmarks among several neurodegenerative diseases. Alpha-synuclein (aSyn), Tau and huntingtin (wildtype and mutant, $25 \mathrm{QH} t \mathrm{tt}$ and $103 \mathrm{QH}$ tt, respectively) were recently shown to be transferred from cell-to-cell through different cellular pathways, thereby contributing to disease progression and neurodegeneration. However, the relative contribution of each of these mechanisms towards the spreading of these different proteins and the overall effect on neuronal function is still unclear.

To address this, we exploited different cell-based systems to conduct a systematic comparison of the mechanisms of release of aSyn, Tau and $\mathrm{Htt}$, and evaluated the effects of each protein upon internalization in microglial, astrocytic, and neuronal cells. In the models used, we demonstrate that $25 \mathrm{QH} t \mathrm{tt}$, aSyn and Tau are released to the extracellular space at higher levels than $103 \mathrm{QHtt}$, and their release can be further augmented with the co-expression of USP19. Furthermore, cortical neurons treated with recombinant monomeric $43 \mathrm{QHtt}$ exhibited alterations in neuronal activity that correlated with the toxicity of the polyglutamine expansion. Tau internalization resulted in an increase in neuronal activity, in contrast to slight effects observed with aSyn. Interestingly, all these disease-associated proteins were present at higher levels in ectosomes than in exosomes. The internalization of both types of extracellular vesicles (EVs) by microglial or astrocytic cells elicited the production of proinflammatory cytokines and promoted an increase in autophagy markers. Additionally, the uptake of the EVs modulated neuronal activity in cortical neurons.

Overall, our systematic study demonstrates the release of neurodegenerative diseaseassociated proteins through similar cellular pathways. Furthermore, it emphasizes that protein release, both in a free form or in EVs, might contribute to a variety of detrimental effects in receiving cells and to progression of pathology, suggesting they may be exploited as valid targets for therapeutic intervention in different neurodegenerative diseases.

Keywords: Alpha-synuclein, Tau, huntingtin, extracellular vesicles, ectosomes, microvesicles, exosomes, spreading, multi-electrode array, neuronal function 


\section{Introduction}

Neurodegenerative diseases are associated with the progressive loss of a variety of brain functions due to the loss of different types of neuronal cells. In spite of characteristic clinical manifestations, these disorders share common neuropathological features and cellular alterations, such as the aggregation and accumulation of disease-related proteins in relatively specific regions of the brain [547]. Alpha-synuclein (aSyn)-containing aggregates are typical in Parkinson's disease (PD) and other synucleinopathies, hyperphosphorylated Tau-containing inclusions are typical in tauopathies, and mutant huntingtin ( $\mathrm{Htt})$-containing inclusions are typical in Huntington's disease (HD) [14, 104, 223].

The progressive accumulation of protein pathology in different brain regions [8-10, 548, 549] and the observation of aSyn Lewy body (LB) pathology fetal dopaminergic neurons grafted in the brains of PD patients led to the hypothesis that the progression of different neurodegenerative diseases may be correlated with the transfer of pathological proteins from sick cells to healthy cells [327, 332]. Consistently, injection of Tau aggregates into the brains of transgenic animals induces pathology along connected brain networks [390, 392-394]. Furthermore, a similar process was hypothesized to occur also in monogenic forms of neurodegenerative diseases after the observation of mutant Htt aggregates within fetal striatal allografts in the brains of HD patients [436].

The old brain is characterized by multi-morbidity, with the simultaneous accumulation of different types of protein pathology [550-552]. In addition, abundant Tau-related pathology can be observed in the brains of PD and HD patients, suggesting that multiple proteins may jointly contribute to the pathophysiology of different neurodegenerative disorders [484, 492494]. Furthermore, the propagation of the pathological proteins between cells and across anatomical connected regions is consistent with the progression patterns described in different neurodegenerative diseases [257, 282, 553]. However, the precise molecular mechanisms underlying the spreading of pathology, and the relative contributions of each of them towards spreading, are still unclear. At a fundamental level, it is also unclear whether cells utilize similar pathways for releasing proteins, such as aSyn, Tau or $\mathrm{Htt}$, and how the released proteins affect neighbouring cells.

Several conventional and unconventional pathways have been implicated in the cellto-cell transfer of proteopathic seeds $[257,282,554]$. The conventional secretory pathway requires the presence of a signal peptide sequence in the secreted protein that is then translocated to the endoplasmic-reticulum (ER), and sorted through Golgi-derived vesicles that, ultimately, fuse with the plasma membrane, thereby releasing their content/cargoes to the extracellular space [555-558]. Alternatively, cargoes can be sorted and released through 
unconventional pathways, resulting in the release of proteins in free forms, in extracellular vesicles (EVs) [559], or via tunnelling nanotubes, structures that enable the direct transfer of cargoes between connected cells [560].

EVs have been shown to play various roles in the central nervous system (CNS), such as in intercellular communication, or the removal of toxic materials from the cell. In this context, they may also contribute to the transfer of pathogenic proteins in neurodegenerative diseases. EVs may be classified as exosomes or microvesicles (also known as ectosomes). They differ significantly in size, mechanism of biogenesis, and in protein, lipid, and nucleic acid content [295, 310]. Exosomes (30-100 $\mathrm{nm}$ in diameter) originate from the multivesicular bodies (MVBs) and are released upon the fusion of MVB with the plasma membrane. In contrast, ectosomes (100-500 $\mathrm{nm}$ in diameter) are formed by the outward budding of the plasma membrane [310]. Recently, several studies reported that exosomes can contain proteins associated with neurodegenerative diseases and, therefore, may be explored as disease biomarkers [293, $559,561]$. However, the role of ectosomes in the pathogenesis of neurodegenerative diseases, and the general effects of EVs in neuronal activity remain largely unknown [562].

More recently, another unconventional secretion mechanism known as misfoldingassociated protein secretion pathway (MAPS), was described to export misfolded proteins [288, 426]. This mechanism uses the ER-associated deubiquitylase USP19 to preferentially export misfolded cytosolic proteins through the recruitment of proteins to the ER surface for deubiquitylation. These cargoes are then encapsulated into late endosomes and secreted to the extracellular space [288]. After internalization, misfolded proteins may act as seeds to template the misfolding and aggregation of their physiological forms [563-565].

Here, we developed stable cell lines expressing aSyn, Tau and Htt exon 1 (carrying either 25 or 103 polyglutamines, $25 \mathrm{QH} t \mathrm{tt}$ and $103 \mathrm{QH} t \mathrm{tt}$, respectively) fused to EGFP in order to afford a systematic comparison of the various proteins. Our results demonstrate that the different disease-related proteins are released, as free forms and in EVs, at different levels. Overall, 25QHtt-EGFP, aSyn-EGFP and EGFP-Tau were found at higher levels in the cell media than $103 \mathrm{QH}$ tt-EGFP. We observed similar results when these proteins were expressed in primary cortical neurons or expressed without the EGFP tag, suggesting that the process of release to the extracellular space is mainly dependent on the protein properties. Furthermore, we modelled the occurrence of the proteins in the extracellular space, as when proteins are released from cells, and assessed their effect in the spontaneous firing activity and bursting events of mature primary cortical neurons using multi-electrode arrays (MEA). Monomeric 43QHtt induced discernible alterations in the bursting properties of the cells when compared with $23 \mathrm{QH} t \mathrm{tt}$ or with vehicle-treated cells, suggesting detrimental effects of the polyglutamine expansion on neuronal activity. Interestingly, Tau internalization resulted in increased neuronal 
activity, with cells exhibiting shorter bursts and higher intra-burst spike frequency, in contrast with only minor alterations observed with aSyn. These results suggest that intrinsic properties of aSyn, Tau or Htt present in the extracellular space, and not necessarily the levels of the proteins, modulate their effect on neuronal activity.

Interestingly, aSyn, Tau and Htt are present at higher levels in ectosomes than in exosomes, without altering the overall proteome of the vesicles. Additionally, the internalization of EVs by microglial or astrocytic cells elicited an increase in the levels of IL-6, IL-1 $\beta$ and TNF $\alpha$, pro-inflammatory cytokines. Microglial cells also displayed an increase in p62 and LC3 puncta, suggesting the activation of autophagy for digesting the EVs. Finally, neuronal cells also internalized ectosomes and exosomes enriched in aSyn, Tau or $\mathrm{Htt}$ and, consequently, exhibited cell bursting irregularities that, overall, correlated with the type of EV used.

Our results indicate that common cellular mechanisms may be used for the transfer of aSyn, Tau and Htt between different cell types. Interestingly, we report that these proteins are handled differently depending on the receptor cell. We posit that the identified similarities and differences between the release and extracellular effects of the three proteins suggest the need for careful consideration of possible targets for therapeutic intervention in different diseases.

\section{Results}

\section{aSyn, Tau and Htt are released to the extracellular space in different cell models}

To evaluate the release of aSyn, Tau and $\mathrm{Htt}$ to the extracellular space, we established stable HEK cell lines expressing the different disease-related proteins fused to EGFP (Figure 1). In particular, we expressed two biologically- relevant $\mathrm{N}$-terminal exon $1 \mathrm{Htt}$ fragments with either 25 or 103 polyglutamines (representing wild-type and mutant $\mathrm{Htt}, 25 \mathrm{QHtt}-\mathrm{EGFP}$ and 103QHtt-EGFP, respectively) (Figure 1A-B). While 25QHtt-EGFP, aSyn-EGFP and EGFP-Tau expression was mainly diffused in the cytoplasm, 103QHtt-EGFP accumulated in inclusions in the nucleus and throughout the cell (Figure 1A). Importantly, the use of the same cell type expressing the different proteins enabled us to directly compare the effect of the cellular machinery on protein release. We found that aSyn, Tau and Htt were differentially released to the extracellular space (Figure 1B). Cell lysates and conditioned media were assessed by SDS-PAGE, and protein levels were normalized to total protein levels using Memcode (Figure $1 \mathrm{~B})$. In particular, the levels of $25 \mathrm{QH} t \mathrm{tt}-\mathrm{EGFP}$ in the media were higher than those of $103 \mathrm{QH} t \mathrm{tt}-$ EGFP, aSyn-EGFP or EGFP-Tau (Figure 1B). 
A

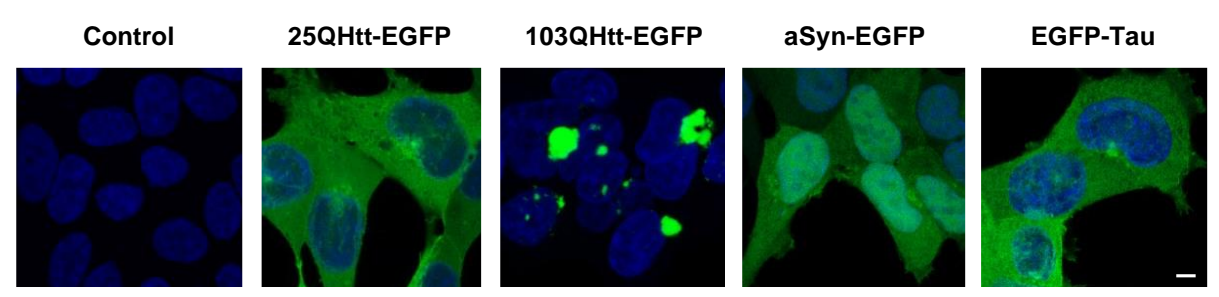

B
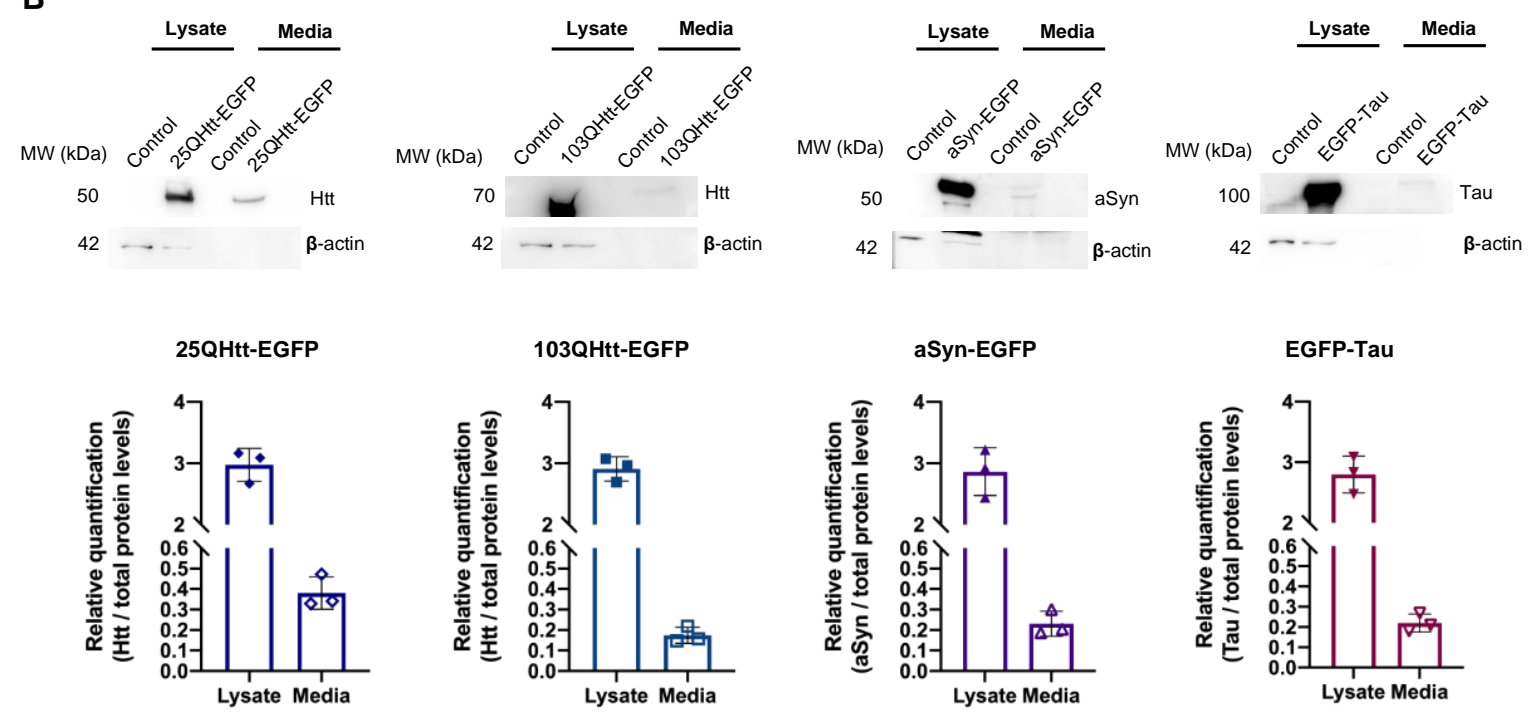

C
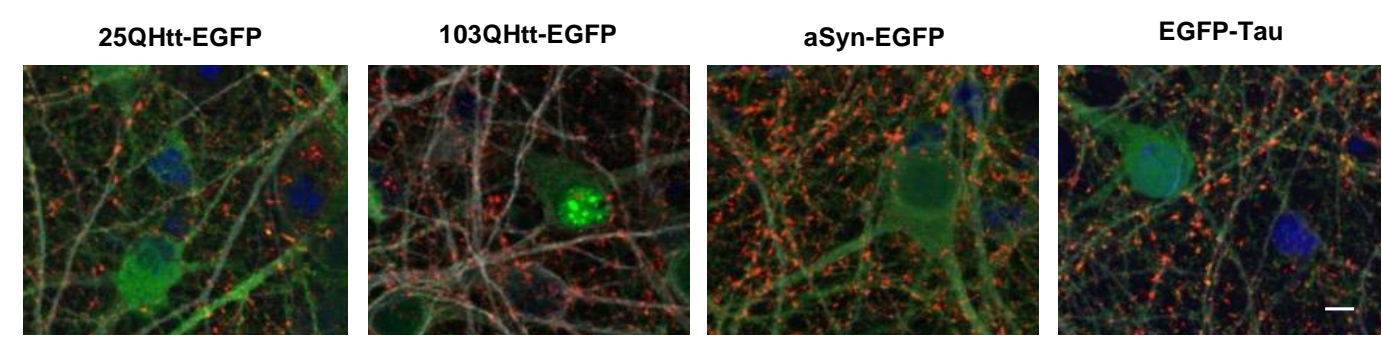

D

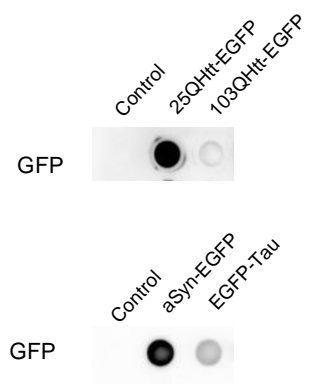

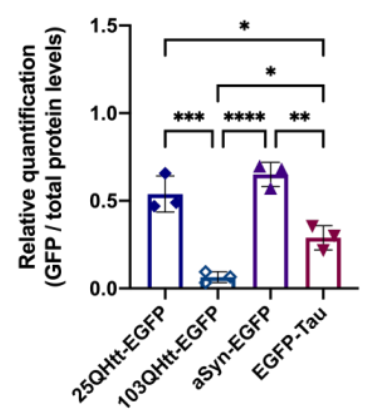

E

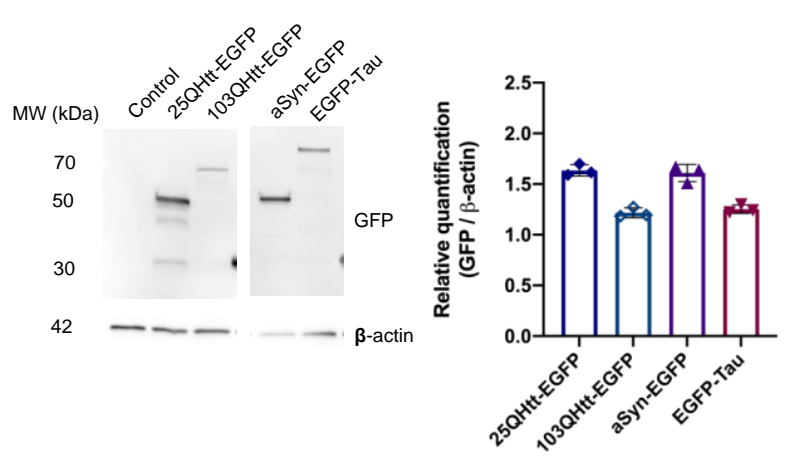

Figure 1. aSyn, Tau and $\mathrm{Htt}$ are released to the extracellular space. (A) Representative images of HEK cells stably expressing 25QHtt-EGFP, 103QHtt-EGFP, aSyn-EGFP, or EGFP-Tau. Scale bar 5 $\mu \mathrm{m}$. (B) Immunoblots showing the protein levels in cell lysates and in the cell media of the different cell lines. Quantifications were normalized to total protein levels using MemCode. (C) Representative images of primary cortical neurons infected with lentiviral vectors encoding 25QHtt-EGFP, 103QHtt-EGFP, aSyn-EGFP or EGFP-Tau, and immunostained for synaptophysin (red) and MAP2 (grey). Neurons were infected at DIV14 and cultured until DIV19. They were then 
fixed for imaging and the media was collected for further analyses. Scale bar $5 \mu \mathrm{m}$. (D) Dot blot analyses of the protein levels released to the cell media of cells expressing the different proteins. Quantifications were normalized to total protein levels using Memcode. (E) Immunoblots showing the EGFP levels in whole-cell lysates. Quantifications were normalized to $\beta$-actin. Data from at least three independent experiments for each condition. Significant differences were assessed by one-way ANOVA followed by multiple comparisons with significance between groups followed by Bonferroni correction. Differences were considered to be significant for values of $\mathrm{p}<0.05$ and are expressed as mean $\pm \mathrm{SD},{ }^{*} \mathrm{p}<0.05,{ }^{* *} \mathrm{p}<0.01,{ }^{* * *} \mathrm{p}<0.001,{ }^{* * * *} \mathrm{p}<0.0001$. See also Supplementary Figure 1.

In addition, we evaluated the release of aSyn, Tau and $\mathrm{Htt}$ in primary neuronal cultures (Figure 1C-E). Cortical neurons were infected with lentiviral vectors encoding for the various proteins (DIV14), to ensure homogeneous transduction (Figure 1C). Cell media was collected at DIV19 and applied onto a dot blot system to assess the presence of the different proteins in the extracellular space (Figure 1D). We observed that 25QHtt-EGFP was released at higher levels than 103QHtt-EGFP. Overall, 25QHtt-EGFP and aSyn-EGFP were released at higher levels compared with the other proteins (Figure 1D), but these differences were not simply associated with the expression levels in the cells (Figure 1E).

To rule out that secretion was associated with the presence of the EGFP tag, we expressed untagged aSyn, Tau and Htt proteins in HEK cells (Supplementary Figure 1). Consistently with the previous data, we observed higher levels of 22QHtt, aSyn and Tau in the cell media, and lower levels of $72 \mathrm{QHtt}$ (Supplementary Figure 1B). Furthermore, cells did not exhibit signs of toxicity-induced permeabilization, as shown by the lactate dehydrogenase (LDH) cytotoxicity assay (Supplementary Figure 1C - E).

These results demonstrate that aSyn, Tau and Htt are released to the extracellular space at different levels, independently of the cell model used.

\section{USP19 promotes the secretion of disease-related proteins}

Recently, USP19 has been proposed to regulate protein secretion [288]. To investigate whether MAPS was involved in the release of aSyn, Tau or Htt, we co-expressed USP19 or the catalytic inactive form USP19 C506S with aSyn, Tau or Htt using the HEK stable cell lines we generated above (Figure 2). Interestingly, USP19 significantly increased the secretion of 25QHtt-EGFP after 24 hours (Figure 2A). We also observed a trend towards an increase in the secretion of 103QHtt-EGFP, aSyn-EGFP and EGFP-Tau, but this did not reach statistical significance (Figure 2A). No effects were observed when we co-expressed the catalytic inactive form USP19 C506S, confirming the effects observed were associated with the activity of USP19. Cells did not exhibit signs of toxicity-induced permeabilization (Figure 2B). 

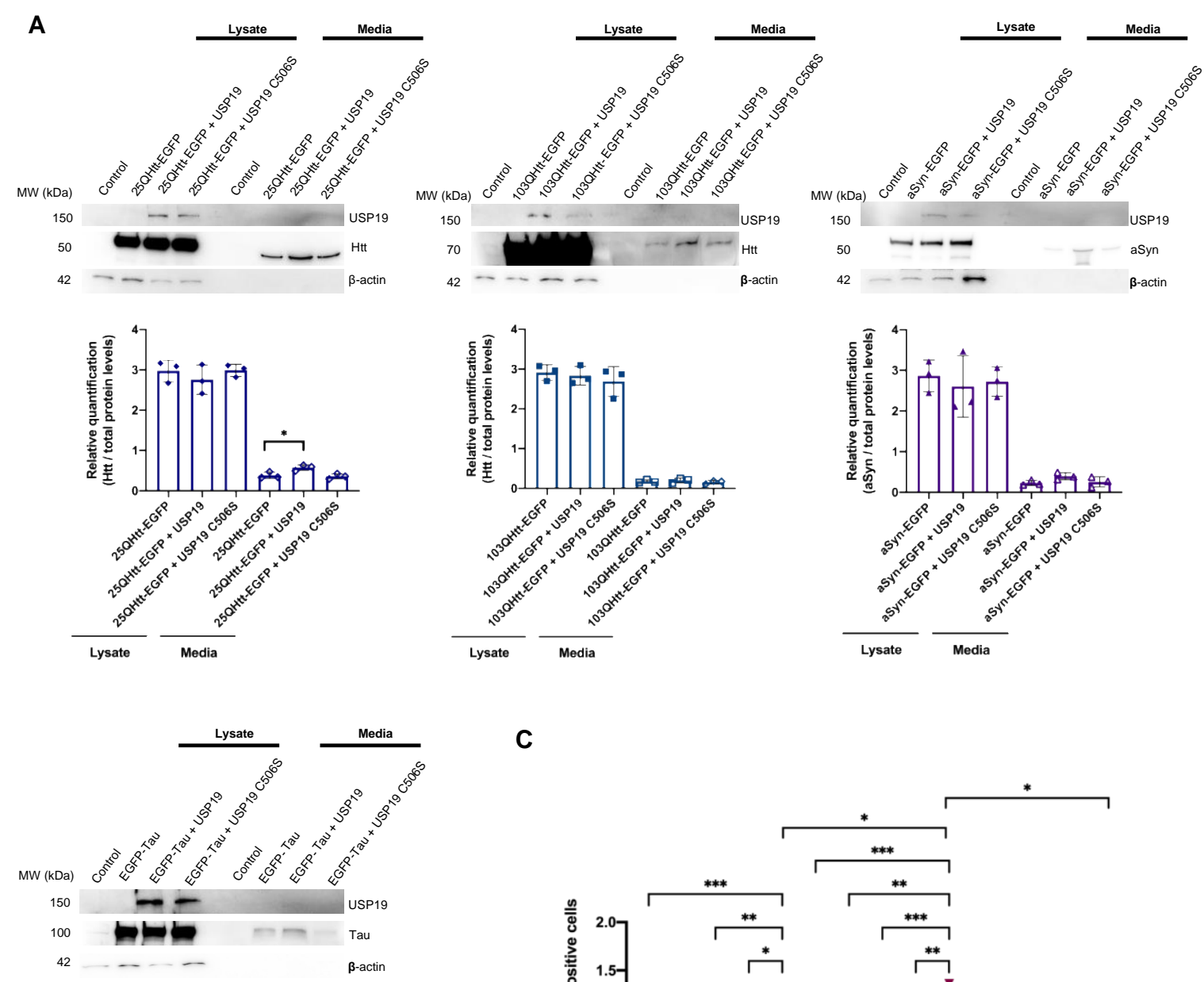

C
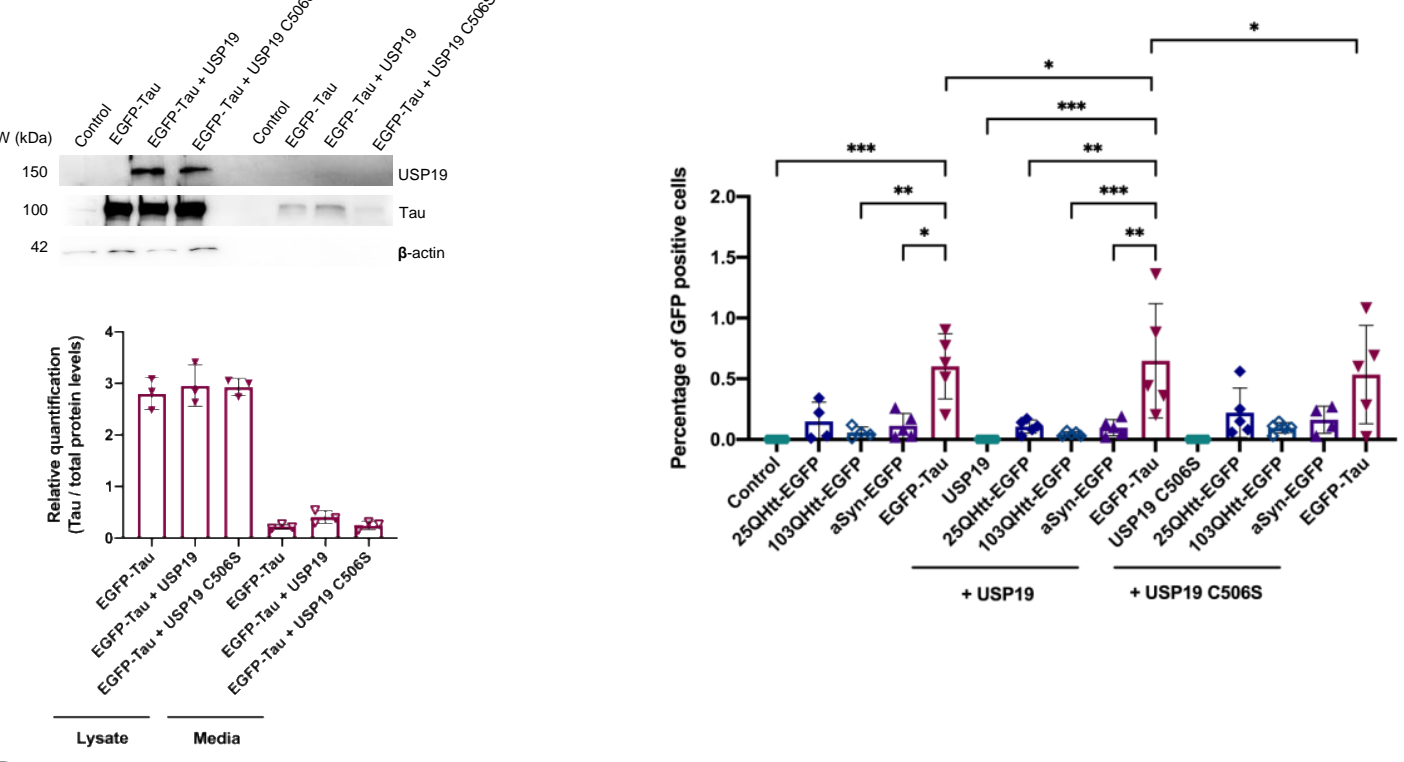

B

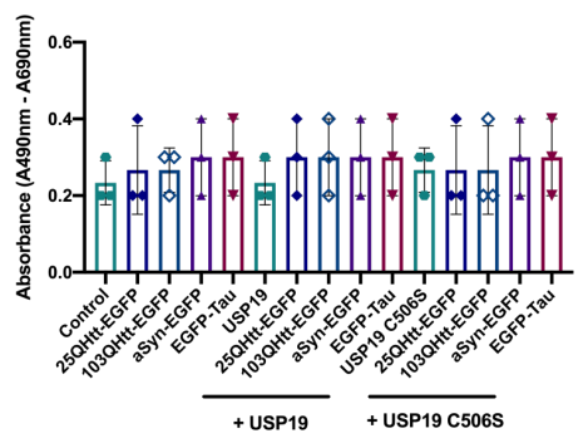

Figure 2. USP19 increases the release of aSyn, Tau or $\mathrm{Htt}$ to the extracellular space. (A) Immunoblots showing protein levels in cell lysates and released to the cell media of cells expressing the different proteins. HEK cell stably 
expressing 25QHtt-EGFP, 103QHtt-EGFP, aSyn-EGFP and EGFP-Tau were transfected with USP19 or with the catalytic inactive form USP19 C506S. Quantifications were normalized to total protein levels using MemCode. (B) LDH measurements confirm the absence of cell toxicity and cell death in the experiments. (C) Tau is more strongly internalized by naïve cells. Percentage of EGFP positive cells after incubation with media from cells co-expressing 25QHtt-EGFP, 103QHtt-EGFP, aSyn-EGFP or EGFP-Tau together with USP19 or USP19 C506S for 24 hours. Cell counting was performed using flow cytometry. Data from at least three independent experiments for each condition. Significant differences were assessed by one-way ANOVA followed by multiple comparisons with significance between groups corrected by Bonferroni procedure. Differences were considered to be significant for values of $p<0.05$ and are expressed as mean $\pm S D,{ }^{*} p<0.05,{ }^{* *} p<0.01,{ }^{* * *} p<0.001,{ }^{* * \star *} p<0.0001$.

Next, we tested whether the secreted proteins could be internalized by naïve cells. For this, the cell media from the different stable cell lines was collected after 24 hours, shortly centrifuged to deplete possible floating cells present in the media, and then added to naïve HEK cells for 72 hours. The number of EGFP-positive cells was analysed using flow cytometry, and we found a higher percentage of EGFP-Tau positive cells compared with the other conditions (Figure 2A, 2C). Strikingly, EGFP-Tau was not the protein released at higher levels to the cell media (Figure 2A). Interestingly, we also observed that incubation of naïve cells with media from cells co-expressing EGFP-Tau and USP19 resulted in a higher percentage of positive cells, indicating higher internalization levels when compared with the other proteins (Figure 2C).

Together, these results indicate that the levels of protein secretion are not directly correlated with the levels of internalization by receiving cells and may, instead, depend on intrinsic properties of the proteins and on the pathways involved in protein uptake.

\section{Disease-related proteins are present at higher levels in ectosomes}

Previous research indicates that aSyn, Tau and $\mathrm{Htt}$ can be secreted in association with EVs [293, 324]. To assess the contribution of ectosomes and exosomes towards the transfer of aSyn, Tau and Htt, we used an optimized differential ultracentrifugation protocol to purify these EVs from the cell media of HEK cells stably expressing the different proteins (Figure 3, Supplementary Figure 2) [562]. Staining of ectosomal and exosomal fractions showed a similar total protein profile that, as expected, was distinct from that of the whole-cell lysate (Supplementary Figure 2A). Electron microscopy (EM) imaging confirmed the greater diameter of ectosomes in comparison to exosomes, and their characteristic cup-shape derived from the ultracentrifugation protocol (Supplementary Figure 2B). In addition, the size distribution and concentration of the two EV types was further validated using Nanosight (Supplementary Figure 2C). While ectosomes presented a diameter of $\sim 140 \mathrm{~nm}$, the diameter of exosomes was $\sim 60 \mathrm{~nm}$ (Supplementary Figure $2 \mathrm{C}$ ). Conventional exosomal protein markers such as alix, 
flotillin-1 or TSG101 were clearly enriched in the exosomal fraction, whereas the ectosomal fraction was enriched in annexin-A2 and annexin-A5 (evaluated using immunoblot and mass spectrometry) (Figure 3A, Supplementary Figure 2D). The ER and Golgi markers calnexin and GM130, respectively, were not detected, confirming the high purity of the isolated EVs (Supplementary Figure 2D).

Interestingly, aSyn, Tau and $\mathrm{Htt}$ were detected at higher levels in ectosomes than in exosomes (EGFP levels were normalized to the total protein levels in the immunoblot using MemCode) (Figure 3A). These results were further confirmed using antibodies specific for aSyn, Tau or Htt, and by mass spectrometry analyses (Supplementary Figure 3). We also detected S129 phosphorylation of aSyn, a posttranslational modification (PTM) typically associated with pathology, in the lysates of aSyn-EGFP expressing cells, but not in the EV fractions (Supplementary Figure 3A). Surprisingly, ectosomes and exosomes containing 25QHtt-EGFP, 103QHtt-EGFP, aSyn-EGFP or EGFP-Tau presented similar proteomic signatures when compared with EVs purified from control cells (Supplementary Figure 3B-C).

Immuno-EM experiments demonstrated the presence of the different disease-related proteins in the cytoplasm, as expected, but also near to the plasma membrane, implying their availability to be incorporated in ectosomes (Figure 3B). Next, to assess the biochemical state of aSyn, Tau and $\mathrm{Htt}$ in the cell media and in EVs, the different samples were applied onto a native gel (Figure $3 \mathrm{C}$ ). Overall, cell media presented greater levels of high molecular species when compared with the EV fractions, possibly due to the higher levels of 25QHtt-EGFP, 103QHtt-EGFP, aSyn-EGFP or EGFP-Tau present in the cell media. Furthermore, ectosomes containing the disease-related proteins presented a stronger smear when compared with exosomes.

These results highlight the prominent role ectosomes, and not only exosomes, may play in the release of disease-related proteins to the extracellular space. 
A
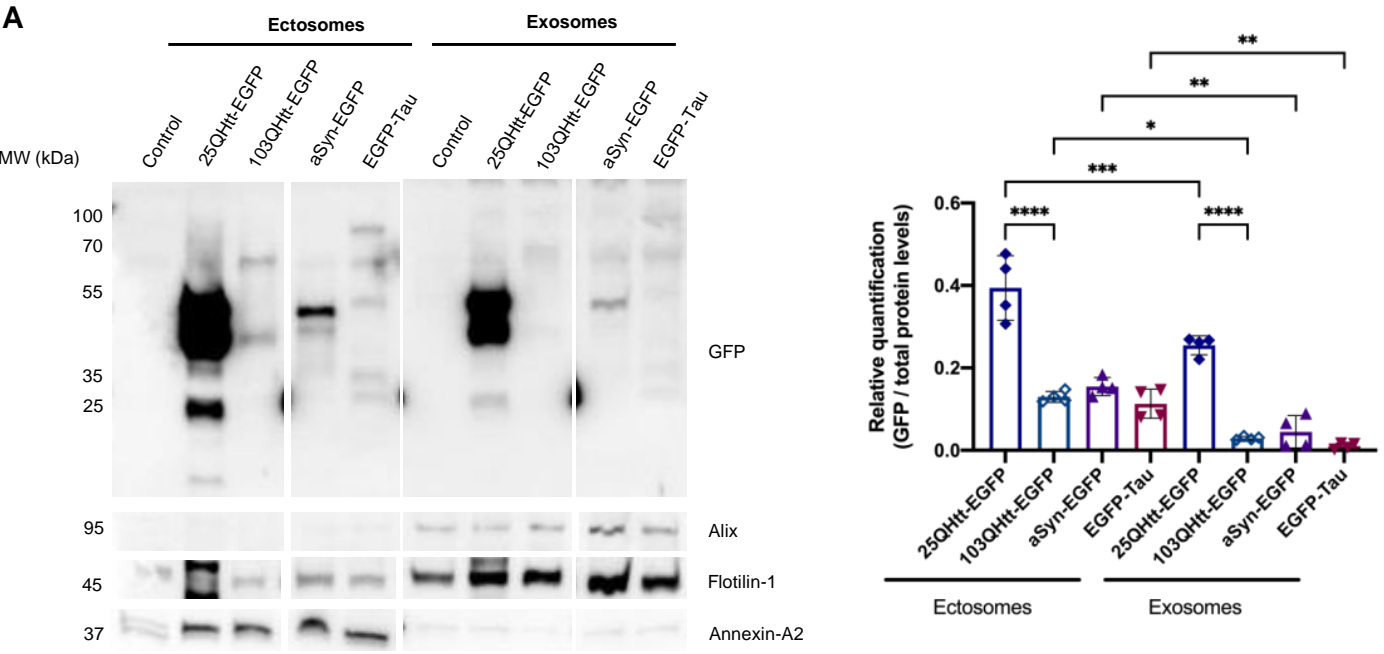

B

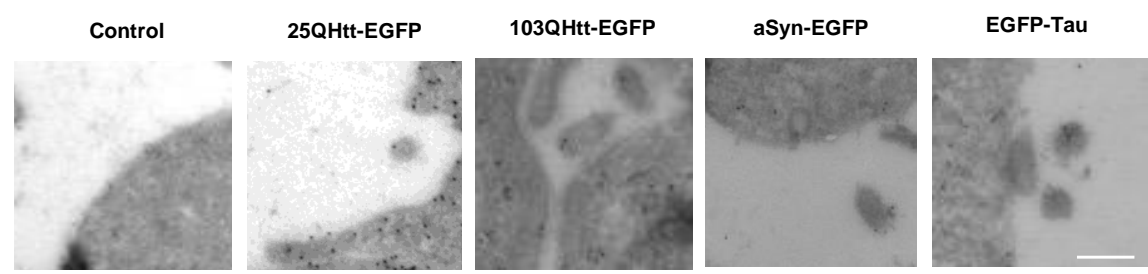

C
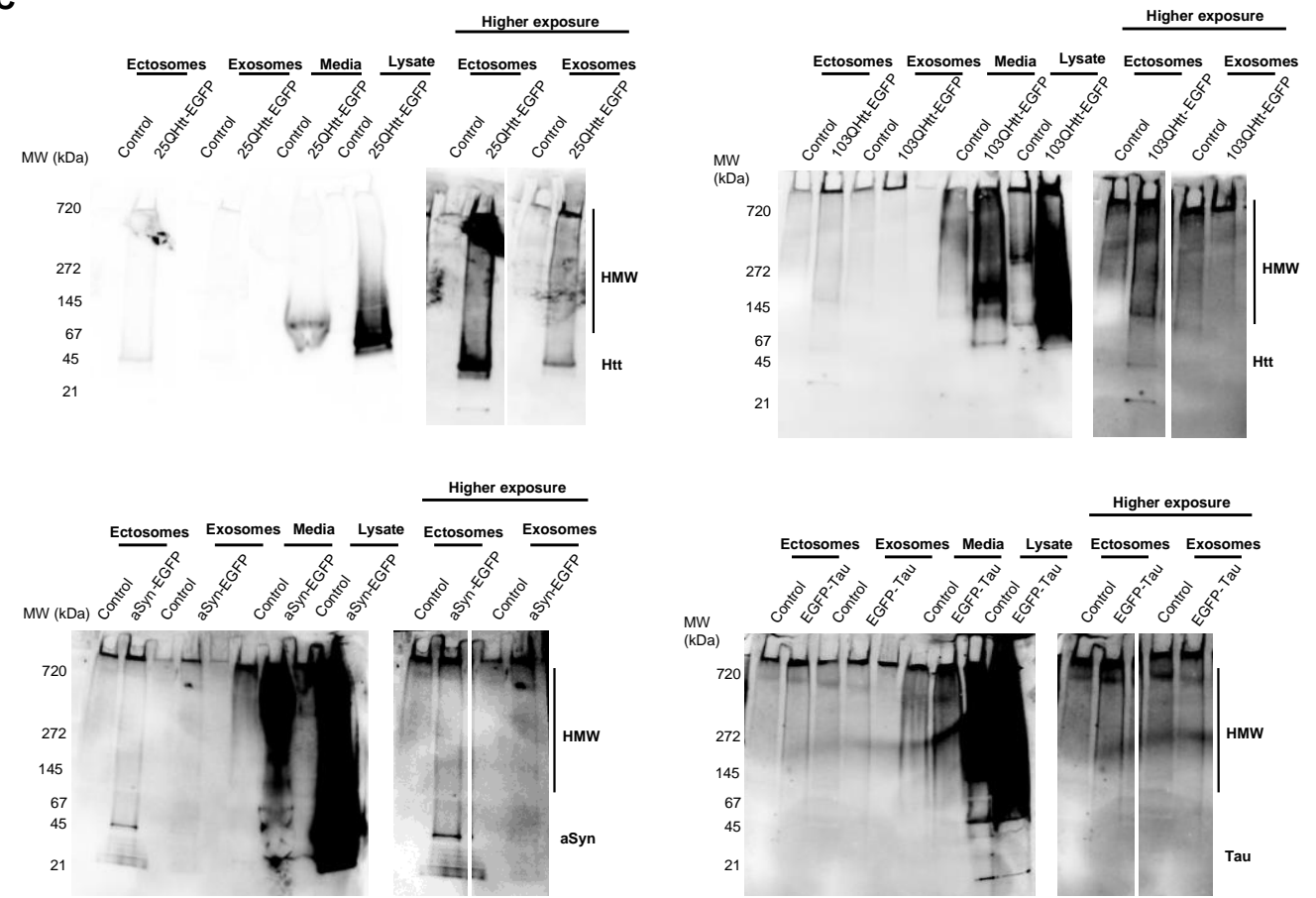

Figure 3. Disease-related proteins are enriched in ectosomes. (A) Immunoblots of ectosomal and exosomal fractions purified from the media of HEK cells stably expressing 25QHtt-EGFP, 103QHtt-EGFP, aSyn-EGFP or EGFP-Tau for 24 hours. Equal amounts of protein were separated on SDS-PAGE gels, and membranes were incubated with the indicated antibodies. Protein levels were normalized to total protein levels using Memcode. (B) Representative immunoelectron microscopy images of HEK cells stably expressing 25QHtt-EGFP, 103QHtt-EGFP, aSyn-EGFP or EGFP-Tau immunolabelled for EGFP, as a common marker (scale bar $200 \mathrm{~nm}$ ). (C) Ectosomes and exosomes containing disease-related proteins have high molecular weight (HMW) species. Ectosomal and exosomal fractions, cell media and whole-cell lysates of HEK cells stably expressing 25QHtt-EGFP, 103QHtt-EGFP, 
aSyn-EGFP or EGFP-Tau. Equal amounts of the proteins were separated on native gels, and membranes were incubated with the indicated antibodies. Data from at least three independent experiments for each condition. Significant differences were assessed by one-way ANOVA followed by multiple comparisons with significance between groups corrected by Bonferroni procedure. Differences were considered to be significant for values of $\mathrm{p}<0.05$ and are expressed as mean $\pm \mathrm{SD},{ }^{*} \mathrm{p}<0.05,{ }^{* *} \mathrm{p}<0.01,{ }^{\star \star *} \mathrm{p}<0.001,{ }^{\star \star * *} \mathrm{p}<0.0001$. See also Supplementary Figures 2-3.

\section{EVs containing aSyn, Tau and $\mathrm{Htt}$ are internalized by glial cells and induce the production of pro-inflammatory cytokines}

Microglial and astrocytic cells produce neuroinflammatory cytokines and appear to be involved in the spreading in neurodegenerative diseases [298, 566, 567]. Therefore, we then asked whether ectosomes and exosomes containing aSyn, Tau and Htt were internalized by different brain cell types, and whether the disease-associated protein present in the vesicles would alter the responses elicited (Figure 4).

Primary microglial cells were treated with $10 \mu \mathrm{g} / \mathrm{mL}$ of ectosomes and exosomes purified from the media of cells expressing 25QHtt-EGFP, 103QHtt-EGFP, aSyn-EGFP or EGFP-Tau (Figure 4, Supplementary Figure 4-5). Furthermore, cell were also exposed to the bacterial endotoxin lipopolysaccharide (LPS) as a pro-inflammatory stimulus [568]. After 24 hours of treatment, EGFP-labelled ectosomes and exosomes were found to colocalize with the microglial marker lba1 in the cell cytoplasm, indicating EVs were internalized (Figure 4A, Supplementary Figure 4A). Interestingly, EVs containing 103QHtt-EGFP were less internalized, or degraded more rapidly, when compared with EVs containing 25QHtt-EGFP (Figure 4B). Overall, the internalization ratio was similar for all ectosomes and exosomes tested (Figure 4B). Labelling of EVs with the thiol-based dye Alexa Fluor $633 \mathrm{C} 5$-maleimide further confirmed the previous results (Supplementary Figure 4B-C) [521]. EV internalization resulted in microglia activation, as demonstrated by an increase in cell area and elevated levels of the pro-inflammatory cytokines IL-6 and TNF $\alpha$ (Figure 5B-D). These results were independent of the presence of aSyn, Tau or Htt in the vesicles (Supplementary Figure 5). Furthermore, EV uptake resulted in an increase in autophagosomes in the cytoplasm (increase in LC3 puncta) and higher p62 levels, indicating autophagy activation [569] (Figure 4B-C). No differences in the levels of iNOS, Iba1, APG5L/ ATG5 or LC3 were observed, and no toxicity was observed after the treatment (Supplementary Figure 5). 
A

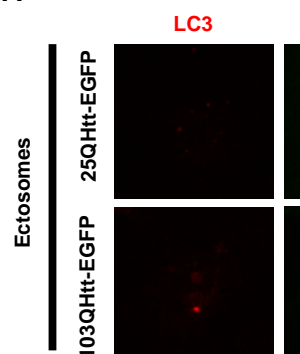

EGFP
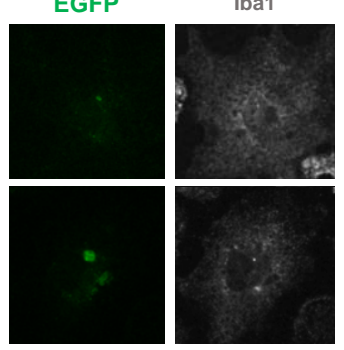

Merge
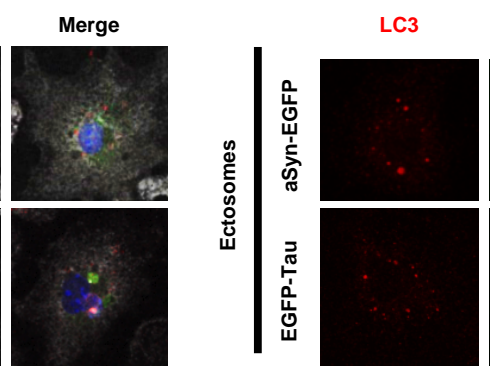

EGFP

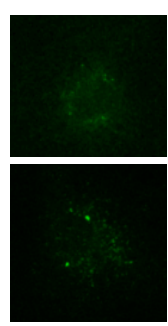

lba1

Merge
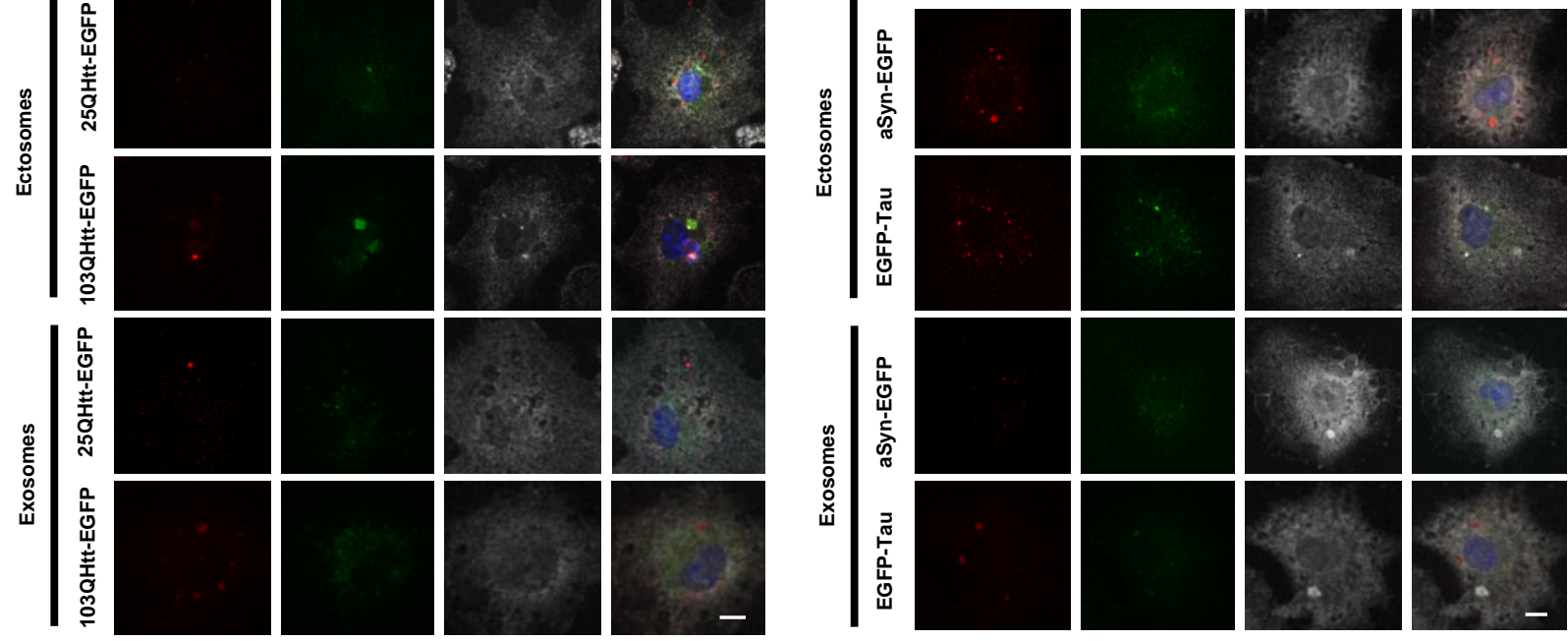

B
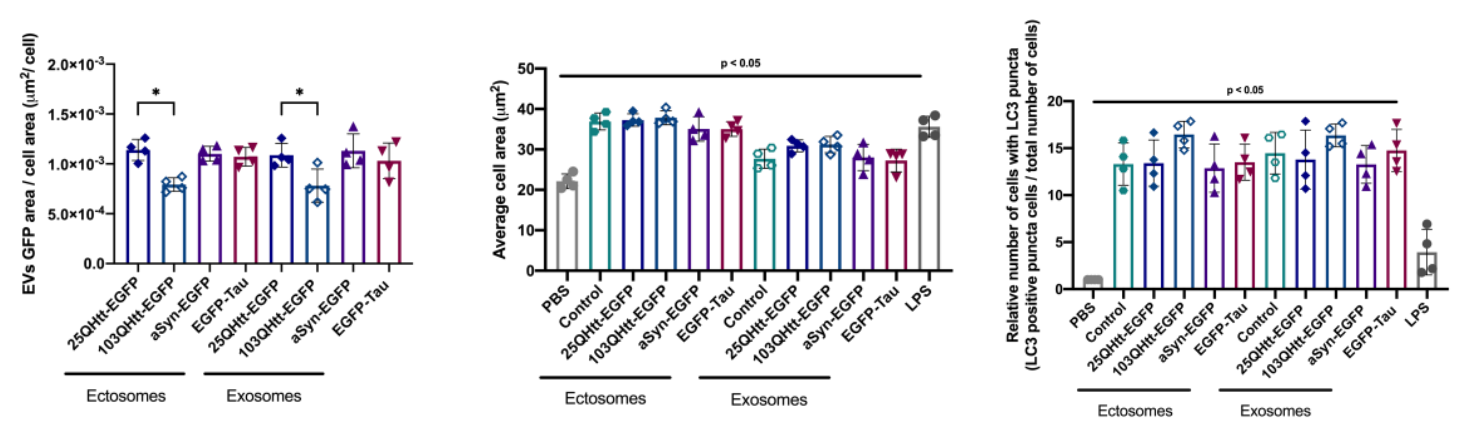

C

D
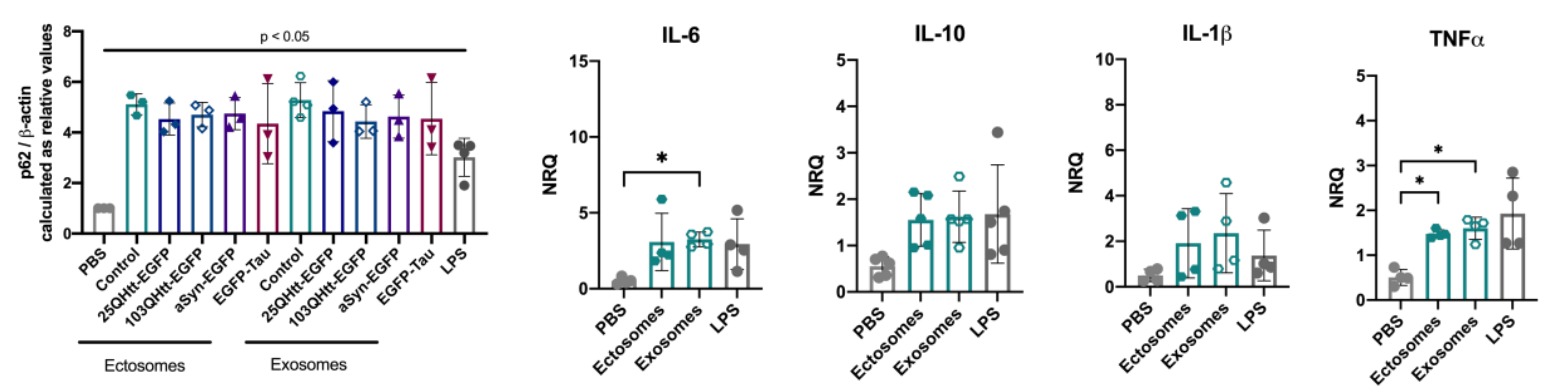

Figure 4. Ectosomes and exosomes containing disease-related proteins are internalized by microglial cells.

(A) Ectosomes and exosomes from cells expressing 25QHtt-EGFP, 103QHtt-EGFP, aSyn-EGFP or EGFP-Tau were applied to microglial cultures at a concentration of $10 \mu \mathrm{g} / \mathrm{mL}$ for 24 hours. Cells were immunoassayed for LC3 (red) and lba1 (grey). Scale bar $10 \mu \mathrm{m}$. (B) EV internalization was evaluated through imaging analysis by measuring EGFP signal and cell area. Microglial cells treated with EVs show an increase in average cell area and number of cells containing LC3-positive puncta. (C) Increase in p62 levels after 24hours of EV treatment. Quantifications were normalized to $\beta$-actin levels. (D) EV treatment resulted in the activation of the pro-inflammatory markers IL- 6 and TNFa in microglia cells after 24hours. Data from at least three independent experiments for each condition. Significant differences were assessed by one-way ANOVA followed by multiple comparisons with significance between groups corrected by Bonferroni procedure. Differences were considered to be significant for values of $p<0.05$ and are expressed as mean $\pm S D,{ }^{*} p<0.05,{ }^{* *} p<0.01$. See also Supplementary Figures $4-5$. 
To further explore the effect of EV internalization in glial cells, primary astrocytes were treated with $20 \mu \mathrm{g} / \mathrm{mL}$ of ectosomes and exosomes purified from cells expressing 25QHttEGFP, 103QHtt-EGFP, aSyn-EGFP or EGFP-Tau (Figure 5, Supplementary Figure 6-7). After 24 hours of treatment, we observed the colocalization of the astrocytic marker GFAP with EGFP-labelled ectosomes and exosomes, confirming internalization (Figure 5A, Supplementary Figure 6A). Interestingly, after internalization, the EGFP signal of the EVs was surrounded by LC3 signal, suggesting a possible engulfment and degradation of EVs in the cells (Figure 5A, Supplementary Figure 6A-C). Quantification of the internalization ratio indicated greater engulfment of ectosomes than exosomes, and these results were further confirmed using dye labelled EVs (Figure 5B, Supplementary Figure 6D). Internalization of ectosomes and exosomes caused an increase in pro-inflammatory cytokines, including IL-6, $\mathrm{IL}-1 \beta$ and TNF $\alpha$, without changing the average cell area (Figure 5B-C). These results were independent of the presence of aSyn, Tau or $\mathrm{Htt}$ in the vesicles (Supplementary Figure 7). No significant differences in the levels of iNOS, APG5L/ ATG5, p62, GFAP or LC3 were observed after EV treatment, and no cytotoxicity was detected (Supplementary Figure 7).

These results indicate that different types of EVs can be internalized in microglial and astrocytic cells, independently of the presence of aSyn, Tau and Htt in the vesicles. Furthermore, EV internalization elicited similar responses, with an increase in inflammatory markers and autophagy activation. 
A
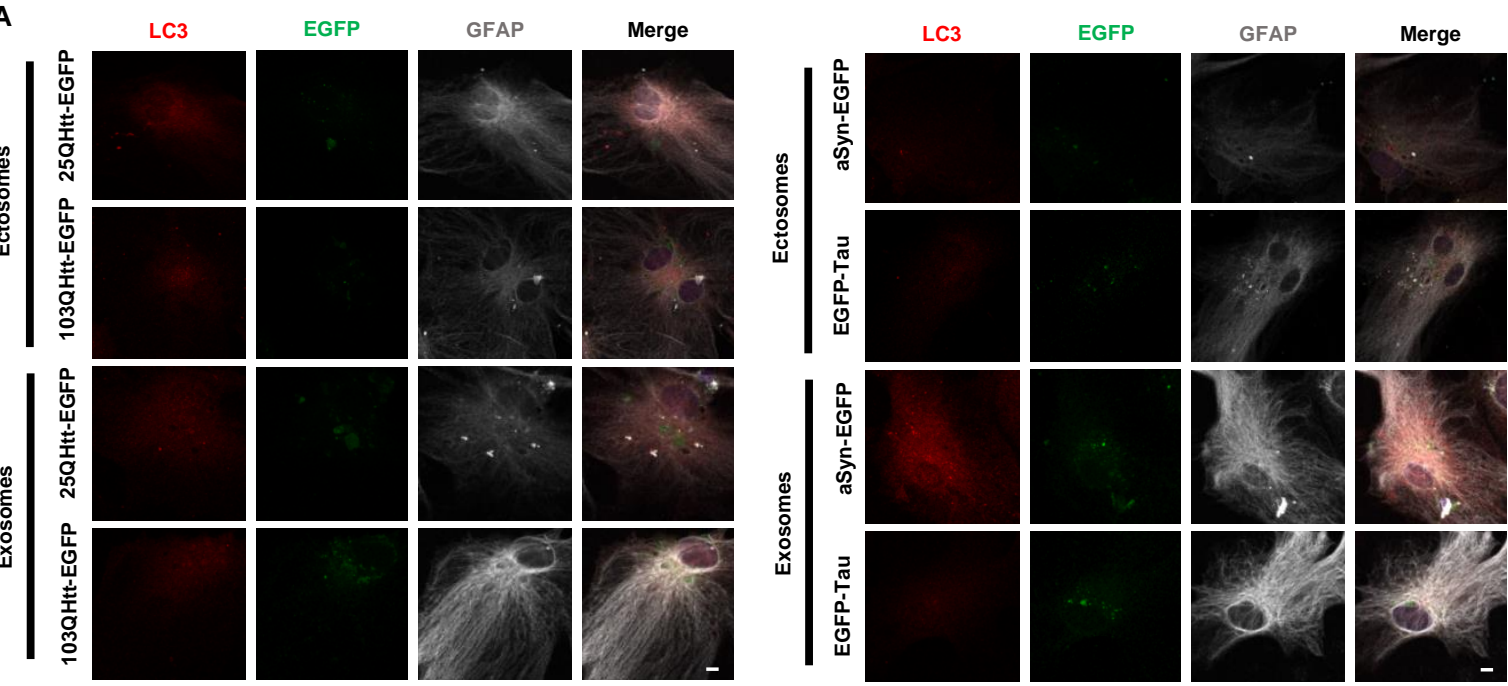

B

C

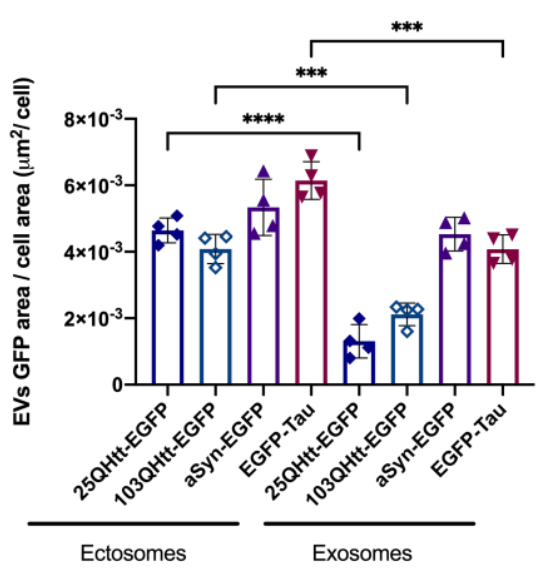

IL-6
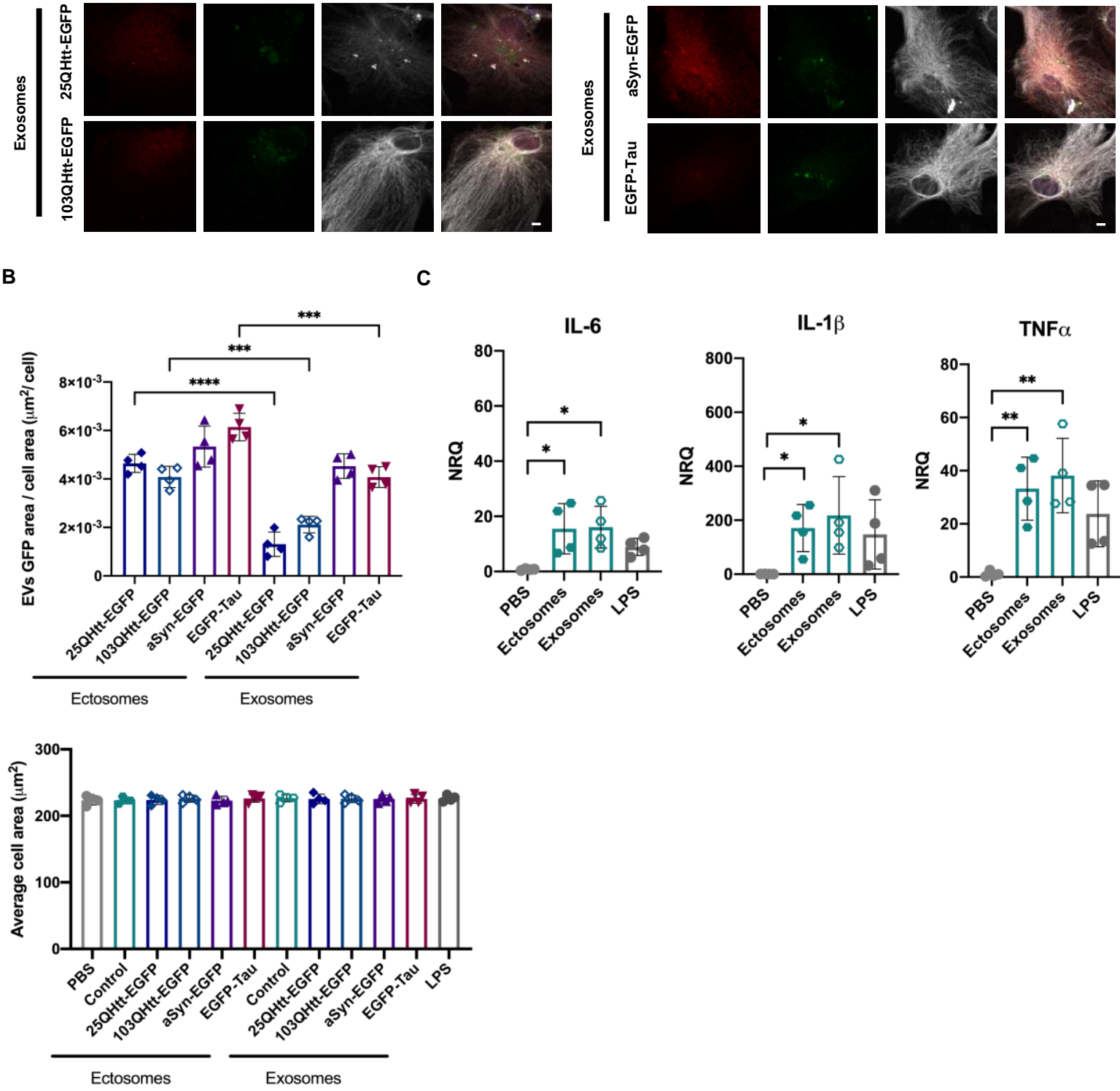

Figure 5. Ectosomes and exosomes containing disease-related proteins are internalized by astrocytic cells.

(A) Ectosomes and exosomes from cells expressing 25QHtt-EGFP, 103QHtt-EGFP, aSyn-EGFP or EGFP-Tau were applied to astrocytic cultures at a concentration of $20 \mu \mathrm{g} / \mathrm{mL}$ for 24 hours. Cells were immunoassayed for LC3 (red) and GFAP (grey). Scale bar $10 \mu \mathrm{m}$. (B) Astrocytic cells treated with EVs do not change average cell area. EVs internalization levels were evaluated through imaging analysis by measuring EGFP signal and cell area. (C) EV treatment resulted in the activation of the pro-inflammatory markers IL-6, IL- $\beta$ and TNF $\alpha$ in astrocytic cells after 24 hours. Data from at least three independent experiments for each condition. Significant differences were assessed by one-way ANOVA followed by multiple comparisons with significance between groups corrected by 
Bonferroni procedure. Differences were considered to be significant for values of $p<0.05$ and are expressed as mean $\pm S D,{ }^{*} p<0.05,{ }^{* *} p<0.01,{ }^{* * *} p<0.001$. See also Supplementary Figures $6-7$.

\section{Treatment with EVs containing aSyn, Tau and Htt modifies spontaneous neuronal activity in cortical neurons}

Since EVs are actively released to the extracellular space by different types of brain cells, we hypothesized that spontaneous neuronal activity might be influenced by the internalization of ectosomes and/or exosomes in neuronal cells [562]. To test this, we treated primary cortical neurons with $20 \mu \mathrm{g} / \mathrm{mL}$ of ectosomes and exosomes purified from cells expressing 25QHtt-EGFP, 103QHtt-EGFP, aSyn-EGFP or EGFP-Tau, at DIV14, for 24 hours to allow internalization of EVs (Figure 6, Supplementary Figure 8).

We observed neuronal cells internalized ectosomes and exosomes at similar levels, and that the EV signal was surrounded by LC3 staining, suggesting the targeting of the EVs for degradation after uptake (Figure 6A-C, Supplementary Figure 8A). The internalization of EVs did not alter the average cell area or caused toxicity and it did not induce significant differences in synaptic and autophagic protein levels (Figure 6D, Supplementary Figure 8CD).

Next, we assessed the effects induced by the EVs on neuronal activity using multielectrode arrays (MEAs) [562]. Primary cortical neurons were cultured in MEA chambers and treated with $20 \mu \mathrm{g} / \mathrm{mL}$ of EVs at DIV14. Firing activity was recorded 24 hours after treatment with ectosomes or exosomes purified from cells expressing 25QHtt-EGFP, 103QHtt-EGFP, aSyn-EGFP or EGFP-Tau (Figure 7). Representative raster plots showed the individual firing activity and bursts events in neuronal cultures for each treatment condition (Figure 7A). We observed a reduction in the mean firing rate after EV internalization, and a decrease in the average spike amplitude for the neurons treated with exosomes (Figure 7B). The assessment of bursting activity parameters showed that, upon treatment with EVs, spike bursts were more irregular and with longer duration (Figure 7C). Overall, exosome internalization resulted in longer inter-burst intervals with reduced intra-burst spiking frequency, and a reduced percentage of spikes within bursts, in contrast to what we observed in neurons treated with ectosomes (Figure 7C). Furthermore, these alterations were more strongly correlated with the EV subtype, and not with the presence of aSyn, Tau or Htt.

Altogether, our results indicate that, after exposure to EVs, the firing of cultured neurons is more irregular, highlighting the potential of ectosomes and exosomes in modifying important aspects of spontaneous neuronal activity. 
A
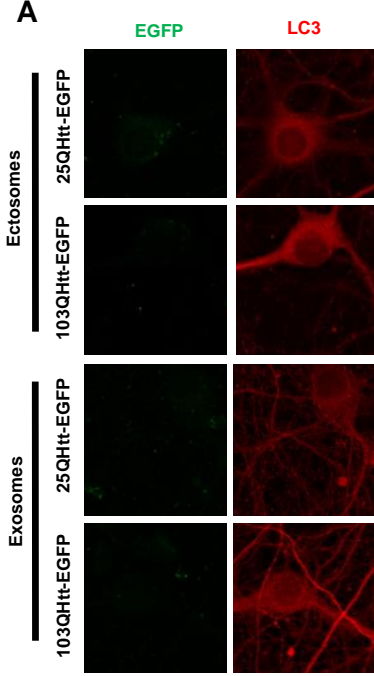

B
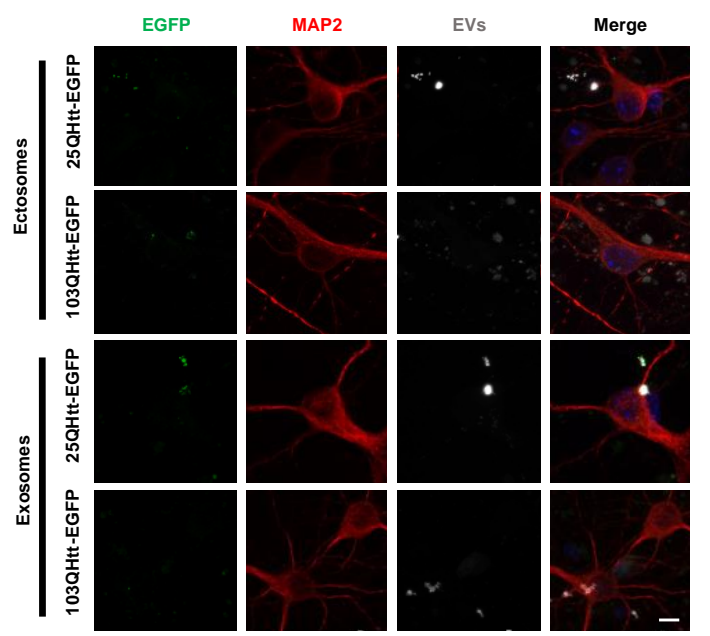

C

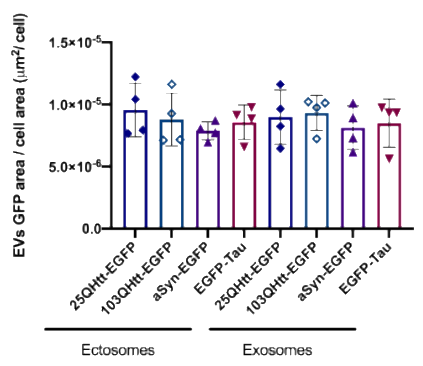

MAP2

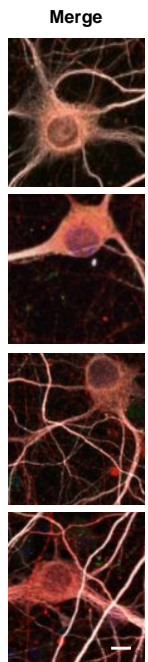

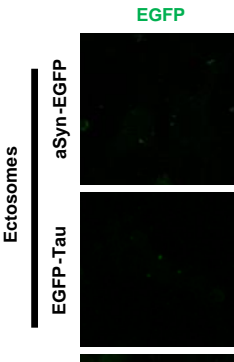
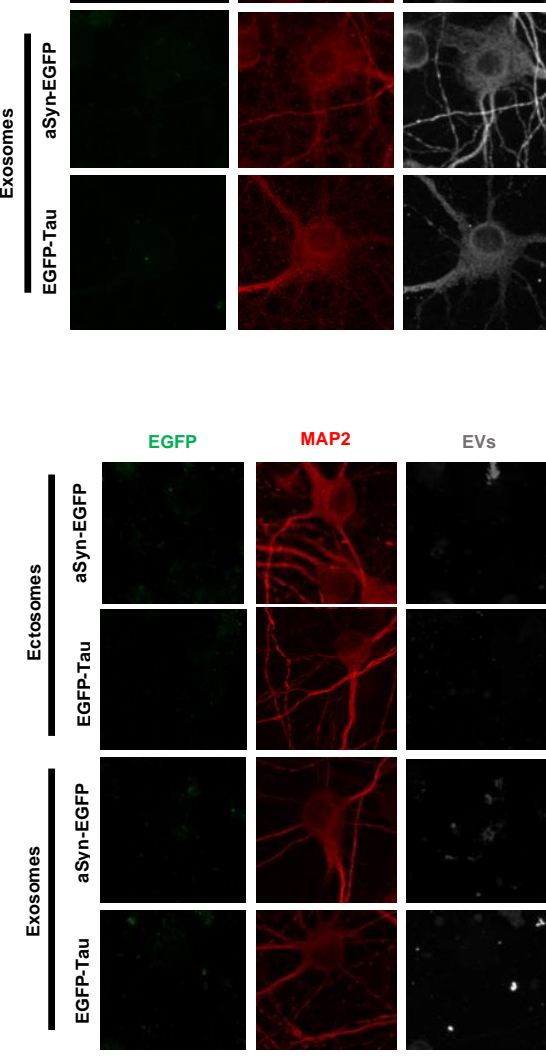

Merge

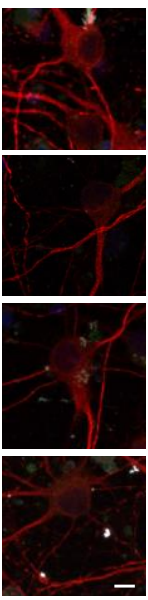

D

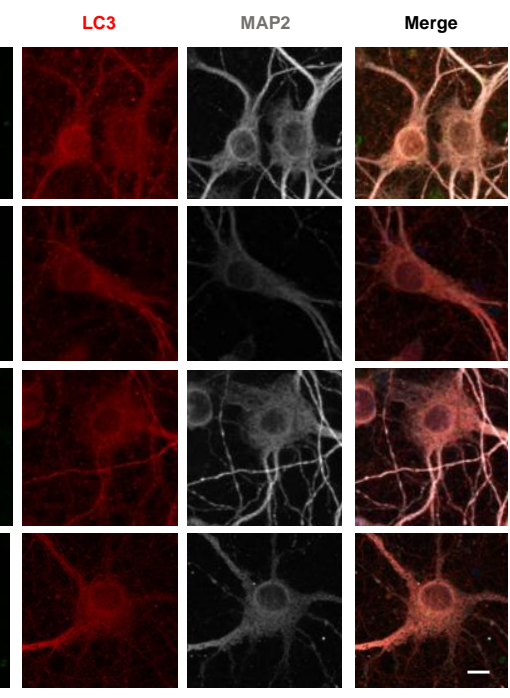

Figure 6. Ectosomes and exosomes containing disease-related proteins are internalized by primary cortical neurons. (A) Ectosomes and exosomes from cells expressing 25QHtt-EGFP, 103QHtt-EGFP, aSyn-EGFP or EGFP-Tau were applied to primary cortical neurons $20 \mu \mathrm{g} / \mathrm{mL}$ for 24 hours. Cells were immunoassayed for LC3 (red) and MAP2 (grey). Scale bar $5 \mu \mathrm{m}$. (B) Ectosomes and exosomes containing 25QHtt-EGFP, 103QHtt-EGFP, aSyn-EGFP or EGFP-Tau were labelled with Alexa Fluor 633 C5-maleimide (grey) and applied to cell cultures at a concentration of $20 \mu \mathrm{g} / \mathrm{mL}$ for 24 hours. Cells were immunostained for MAP2 (red). Scale bar $5 \mu \mathrm{m}$. (C) EV internalization was evaluated through imaging analysis by measuring EGFP signal, dye area, and cell area. (D) Neuronal cells treated with EVs do not change average cell area. Data from at least three independent experiments for each condition. Significant differences were assessed by one-way ANOVA followed by multiple comparisons 
with significance between groups corrected by Bonferroni procedure. Differences were considered to be significant for values of $p<0.05$ and are expressed as mean \pm SD. See also Supplementary Figures 8.

A

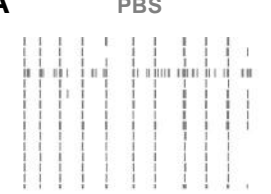

景

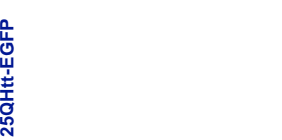

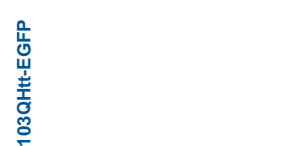

品
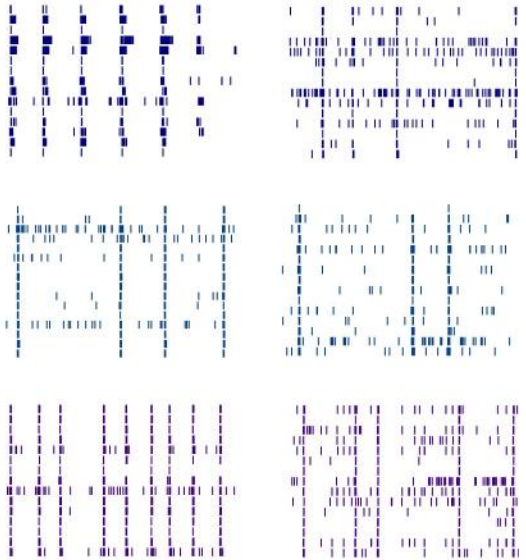

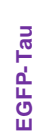
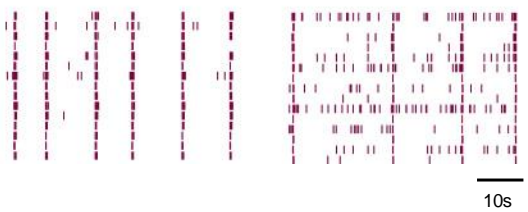

C
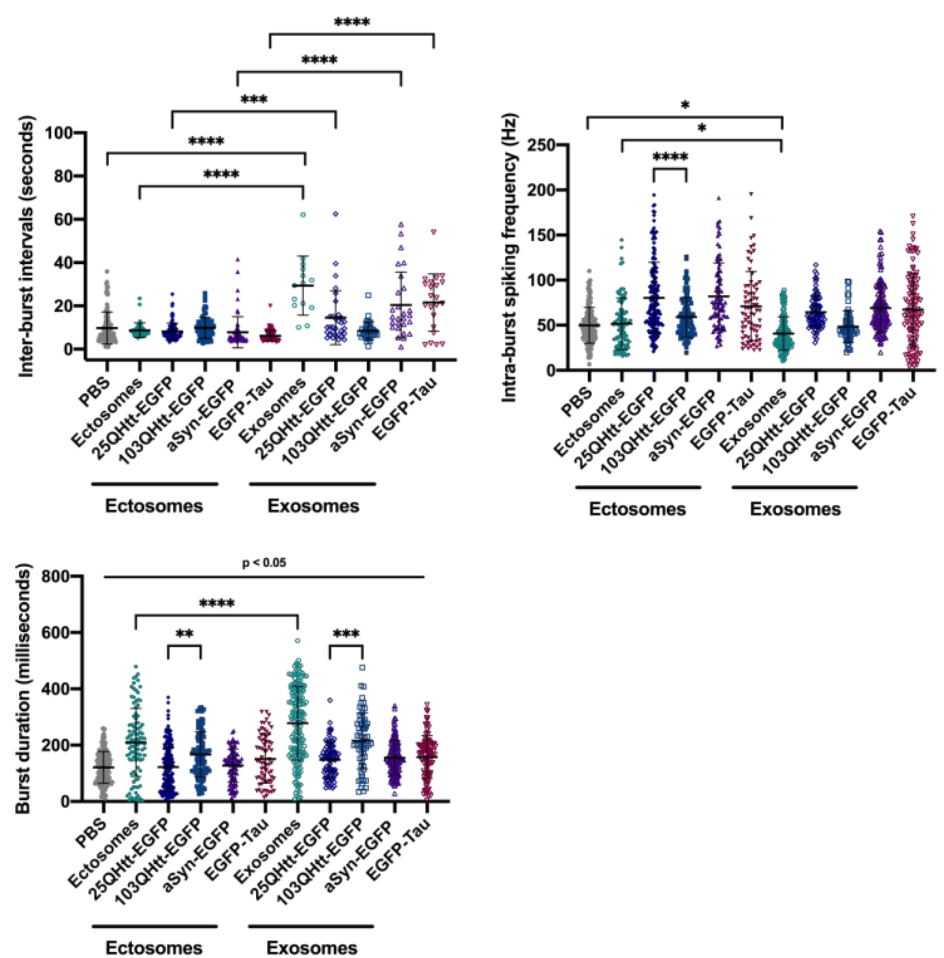

B
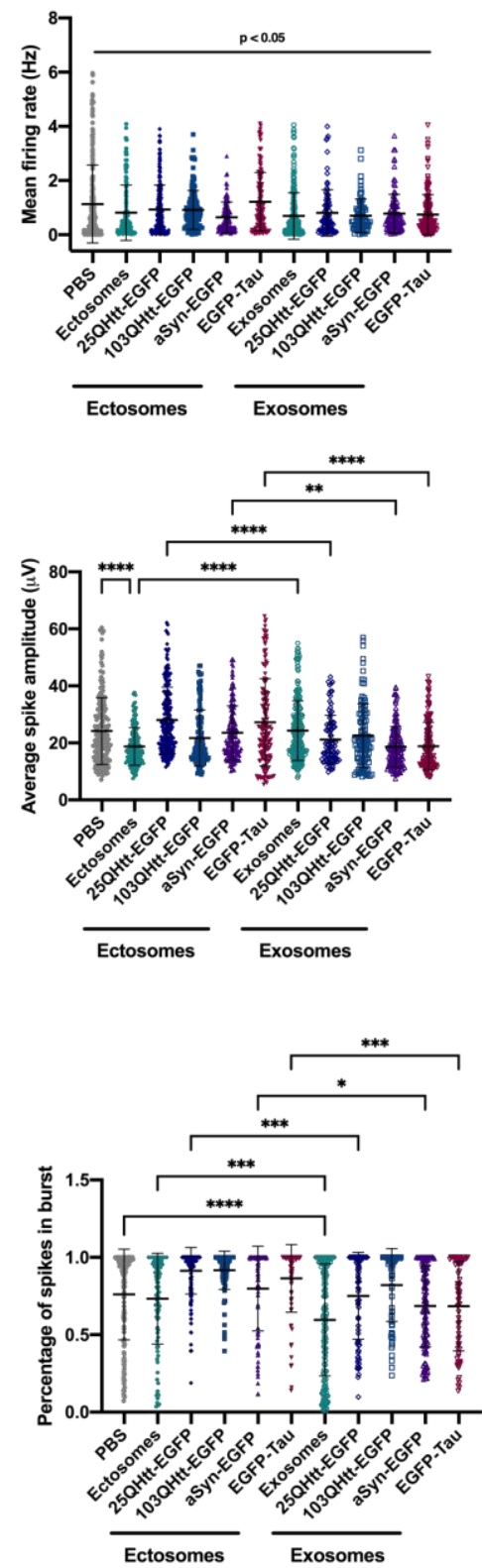

Figure 7. EVs containing disease-related proteins modulate spontaneous activity in primary cortical neurons. (A) Representative raster plots of the spontaneous firing activities recorded from cortical neurons after 
incubation with $20 \mu \mathrm{g} / \mathrm{mL}$ ectosomes and exosomes control or containing aSyn-EGFP, EGFP-Tau, 25QHtt-EGFP and 103QHtt-EGFP at DIV14 for 24 hours, recorded using 60-electrode MEAs. In every block, each row represents one single cell ( 15 cells shown) and each vertical line represents a single spike obtained on DIV15 [scale bar represents 10 seconds (s)]. (B) Quantification of the mean firing rate and average spike amplitude from primary cortical neurons incubated with PBS or disease-related proteins. (C) Bursting properties of the cortical neurons treated with PBS or EVs (inter-burst intervals, intra-burst spiking frequency, percentage of spikes in bursts and burst duration). Data from at least three independent experiments for each condition. Significant differences were assessed by one-way ANOVA followed by multiple comparisons with significance between groups corrected by Bonferroni procedure. Differences were considered to be significant for values of $p<0.05$ and are expressed as mean $\pm \mathrm{SD},{ }^{*} \mathrm{p}<0.05,{ }^{* *} \mathrm{p}<0.01,{ }^{* * *} \mathrm{p}<0.001,{ }^{* \star * *} \mathrm{p}<0.0001$.

\section{Neuronal network activity is differentially modulated by the internalization of monomeric aSyn, Tau or $\mathrm{Htt}$}

Our results indicate the release of wild-type and pathogenic forms of disease-related proteins to the extracellular space. Although these proteins are taken up by cells, it is still not known whether and how they modulate neuronal activity [391, 570-575]. To address the functional effects of free forms of aSyn, Tau and $\mathrm{Htt}$, we treated primary cortical neurons with $100 \mathrm{nM}$ of monomeric aSyn, Tau, $23 \mathrm{QHtt}$ or $43 \mathrm{QHtt}$ for 5 days, starting at DIV14 (this concentration was selected as it demonstrated no detrimental effects, which would be incompatible with long-term observations) (Figure 8) [562].

Representative raster plots and voltage traces show the firing activity and burst events in neuronal cultures treated with PBS (as a negative control), 23QHtt, 43QHtt, aSyn or fulllength Tau (Figure 8A). Overall, neurons treated with monomeric proteins showed a reduction in the mean firing rate. In addition, neurons treated with $\mathrm{Htt}$ exhibited a decrease in the average spike amplitude (Figure 8B). Remarkably, treatment of neuronal cultures with monomeric aSyn or Tau resulted in an increase in the average spike amplitude (Figure 8B). Furthermore, we observed that Tau led to condensed and more intense spike bursting, as demonstrated by bursts with shorter duration and higher intra-burst spike frequency. Also, the percentage of spikes in each burst increased, suggesting that neurons fire in a more regular and synchronized manner (Figure $8 \mathrm{C}$ ). Cells treated with recombinant aSyn exhibited slight alterations in their activity, with longer burst duration and larger intervals between them. Treatment with monomeric $23 \mathrm{QHtt}$ or $43 \mathrm{QH} t \mathrm{tt}$ resulted in bursts with longer intervals and a reduction in the number of spikes within the burst when compared with the control. Interestingly, 43QHtt induced a stronger alteration in the spontaneous neuronal activity with a slight reduction in burst rate and a decrease in the percentage of spikes per burst when compared with $23 \mathrm{QH} t \mathrm{tt}$ or PBS-treated cells. In addition, neurons displayed longer inter-burst 
intervals and higher intra-spike frequency, which was possibly correlated with toxic effects induced by the polyglutamine expansion.

A

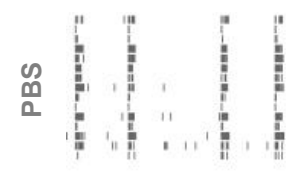

声

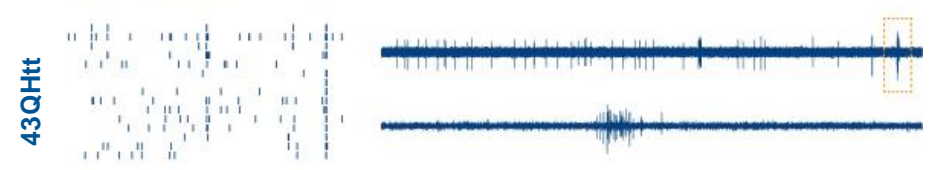

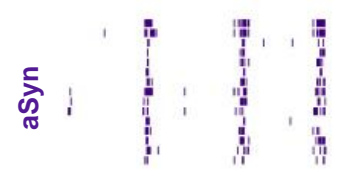

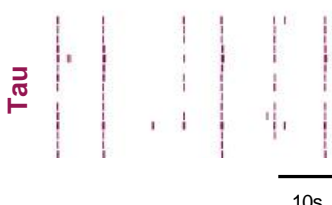

C
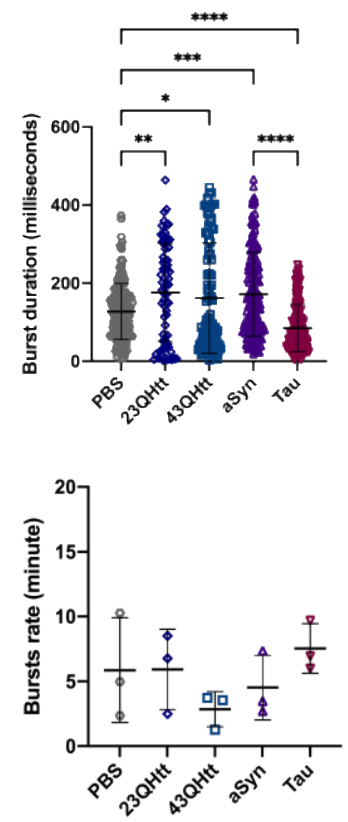

B

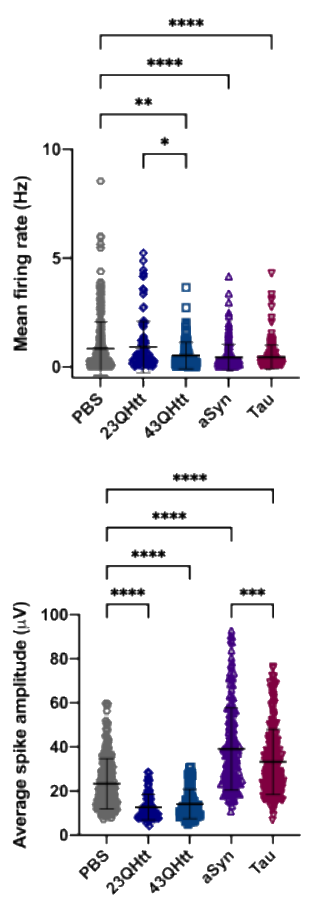

Figure 8. Free monomeric disease-related proteins modulate neuronal activity in primary cortical neurons.

(A) On the left, representative raster plots of the spontaneous firing activities recorded from cortical neurons after incubation with $100 \mathrm{nM}$ of recombinant protein at DIV14 for 5 days, recorded using 60-electrode MEAs. In every block, each row represents one single cell ( 15 cells shown) and each vertical line represents a single spike obtained on DIV19 [scale bar represents 10 seconds (s)]. On the right, representative voltage traces showing the typical firing activity and bursts events in neuronal cultures treated with PBS, monomeric 23Qhtt, 43QHtt, aSyn and Tau (upper 
traces, scale bars represent $60 \mu \mathrm{V}$ and $6 \mathrm{~s}$ ). Closeups of the dashed boxes represent the spikes occurring within a burst ([lower traces, scale bars represent $60 \mu \mathrm{V}$ and 100 milliseconds (ms)]. (B) Quantification of the mean firing rate and average spike amplitude from primary cortical neurons incubated with PBS or disease-related proteins. (C) Bursting properties of the cortical neurons treated with PBS or the disease-related proteins (burst duration, interburst intervals, intra-burst spiking frequency, percentage of spikes in bursts and burst rate). Data from at least three independent experiments for each condition. Significant differences were assessed by one-way ANOVA followed by multiple comparisons with significance between groups corrected by Bonferroni procedure. Differences were considered to be significant for values of $p<0.05$ and are expressed as mean $\pm S D,{ }^{*} p<0.05,{ }^{* *} p<0.01,{ }^{* * *} p<0.001$, ${ }^{\star \star \star *} \mathrm{p}<0.0001$.

Altogether, our results demonstrate that different disease-related proteins induce specific effects on spontaneous neuronal activity.

\section{Discussion}

Several neurodegenerative disease-related proteins appear to be transferred from cellto-cell, contributing for the spreading of pathology and disease progression [257, 282, 554]. However, the basic molecular mechanisms involved in the release of proteins which are, oftentimes, not typical secretory proteins, are still unclear. Likewise, the effect of such proteins once they are in the extracellular milieu, is also unclear. Therefore, deciphering the pathways through which brain cells release, sense, and respond to the extracellular presence of normal and pathological forms of disease-associated proteins is essential. Here, we conducted a systematic comparison of the basic molecular mechanisms involved in the release of three proteins associated with distinct neurodegenerative disorders, aSyn, Tau, and $\mathrm{Htt}$, and of general cell-autonomous responses in different brain cell types. Since these proteins accumulate in different types of brain cells, forming distinct proteinaceous inclusions, and spreading through different neuronal circuits, it is important to establish differences and similarities in the ways they are handled in order to identify specific therapeutic targets for each disease. In our study, exploiting simple, yet tractable, cell systems, we found that aSyn, Tau and $\mathrm{Htt}$ are transferred between cells at different levels, but using overlapping cellular pathways. Importantly, we report that the release of these proteins in a "free form", or in extracellular vesicles, elicits different molecular processes in neighbouring cells.

By taking advantage of stable cell lines expressing aSyn, Tau, 25QHtt and 103QHtt fused to EGFP, as a common denominator, we were able to study and compare different molecular mechanisms involved in the release, and uptake of the various in receptor cells. We demonstrate that 25QHtt-EGFP, aSyn-EGFP and EGFP-Tau are released to the extracellular space at higher levels than 103QHtt-EGFP. Importantly, the presence of the EGFP tag did not alter the release patterns of aSyn, Tau and $\mathrm{Htt}$. 
Our study further implicates the MAPS pathway in the release of a fraction of aSyn, Tau or Htt. In agreement with previous studies, USP19 expression led to a slight increase in the release of aSyn, Tau and Htt $[288,426]$. Interestingly, USP19 promoted a significant increase in the secretion of 25QHtt-EGFP, suggesting a relevant role for this pathway in HD, as it was previously described to interact and regulate mutant $\mathrm{Htt}$ protein levels and promote its aggregation [576, 577].

After release, aSyn, Tau or Htt were found to be internalized by naïve receptor cells. In particular, we observed a significant increase in the percentage of EGFP-positive cells after incubation of naïve cells with media collected from cells expressing EGFP-Tau. Although 25QHtt-EGFP and aSyn-EGFP were more abundant in the cell media than EGFP-Tau, we observed more internalization of this protein in cells. These results suggest that Tau might be more easily, or more rapidly, internalized by cells, or that different pathways might be involved in its uptake when compared with the other proteins. Strikingly, this observation also demonstrates that protein internalization is not a process solely dependent of the protein levels in the exterior space but determined by the type of protein and mechanisms involved in the cellular uptake.

In addition to the release of proteins in free form, several studies have also reported the secretion of disease-related proteins in ectosomes and exosomes [293, 578]. Exosomes are the most extensively studied type of EVs, and are also implicated in the secretion of pathological proteins [324]. However, Tau was also found to be present in ectosomes extracted from culture media from cell models and human cerebrospinal fluid [317, 318], highlighting the relevance of this EV type, and need for further research to address their role in neurodegenerative diseases. In our study, we found that ectosomes purified from the cell media of stable cell lines contained higher levels of aSyn, Tau and Htt than exosomes. Interestingly, we observed that aSyn, Tau and $\mathrm{Htt}$ are present near the plasma membrane, in agreement with their possible incorporation in ectosomes, and release via passive diffusion. Traditionally, ectosome characterization has been challenging due to the lack of specific protein markers. In our study, we highlight the enrichment of annexin-A2 in ectosomes, suggesting this protein as a specific marker for this EV type, as previously described [562].

We also found that incorporation of aSyn, Tau or Htt in EVs did not change the normal vesicle protein composition, suggesting these vesicles are not deregulated when they transport the disease-associated proteins we tested. However, future research will be necessary to determine whether the content of other biomolecules, such as lipids or nucleic acids, is altered, and this then inform on whether the uptake of the EVs by receptor cells may be altered. This is particularly relevant in the context of neurodegenerative diseases, as these vesicles may 
not only play a role in the transmission of proteins but also in signalling cellular alterations taking place during the disease process.

Microglia and astrocytes are two important cell types in the brain that mediate neuroinflammatory processes and, thereby, playing an important role in the pathogenesis of neurodegenerative diseases $[579,580]$. Our study shows that ectosomes and exosomes can be taken up by microglial and astrocytic cells, and that both EV types elicited an increase in pro-inflammatory cytokines (IL-6, IL-1 $\beta$ and TNF $\alpha$ ). Microglial cells adopted an activated phenotype and exhibited LC3 puncta in the cytoplasm and increase in p62 levels, indicating autophagy activation, possible for the clearance of the EVs. Astrocytes also displayed accumulation of EVs in the cytoplasm, with the EGFP signal being surrounded by LC3 staining. Interestingly, microglia cells were more sensitive to EV-treatment: while astrocytes tolerated $20 \mu \mathrm{g} / \mathrm{mL}$, microglia tolerated only up to $10 \mu \mathrm{g} / \mathrm{mL}$ of EV protein. Ectosomes and exosomes containing aSyn-EGFP, EGFP-Tau or 25QHtt-EGFP were taken up at similar levels in microglia, and these were higher than those observed with 103QHtt-EGFP. Indeed, both wildtype and mutant $\mathrm{Htt}$ can influence vesicle transport in the secretory and endocytic pathways through associations with clathrin-coated vesicles [581]. In contrast, astrocytes seemed to, in general, internalize more ectosomes, or degrade exosomes faster. Together, our findings demonstrate that EVs can be targeted by different types of glial cells, and that their uptake and effects are likely correlated with the EV type and content. These findings provide new insights into molecular mechanisms of intercellular communication.

We also observed that ectosomes and exosomes are taken up by primary cortical neurons, as previously described [562]. The internalization ratio was similar for EVs with aSyn, Tau or Htt, but was considerably lower than that observed with microglia and astrocytes. The potential effects of EVs on neuronal network activity are still unclear. In this context, we demonstrate that spontaneous neuronal function can be modulated by ectosomes and exosomes, and that EV internalization is associated with a disruption of the typical synchronized bursting activity, resulting mostly in lower and less organized spiking activity. Interestingly, these alterations are mainly correlated with the EV subtype, and not with the presence of aSyn, Tau or Htt, although slight differences could be perceived between 25QHttEGFP and 103QHtt-EGFP [562].

We also demonstrate that aSyn, Tau or Htt present in the cell media can modify the spontaneous activity in cortical neurons. The use of identical concentrations of monomeric protein allowed us to compare the consequences of aSyn, Tau and Htt internalization, at subcytotoxic concentrations. aSyn function has been associated with synaptic activity through the regulation of the vesicle pool $[36,582-584]$. Interestingly, incubation of cortical neurons with monomeric aSyn resulted in a reduction of the firing rate and in an increase in burst duration, 
without changing the coordinated network activity. These effects contrast with those reported with higher concentrations of extracellular aSyn or with aggregated assemblies that strongly reduce neuronal activity by disrupting synaptic transmission, thereby contributing to neuronal death $[585,586]$. Remarkably, treatment of neurons with monomeric Tau results in increased neuronal activity and in robust and synchronized bursting activity, suggesting that neurons fire in a more regular and synchronized manner. Consistently, full-length monomeric Tau was previously described to be rapidly and efficiently internalized in healthy neurons, implying this might be part of a physiological, and not pathological, process [574]. Treatment of neurons with $23 \mathrm{QH} t \mathrm{tt}$ or $43 \mathrm{QH} t \mathrm{tt}$ resulted in longer burst intervals and a reduction in the synchronized bursting activity. As expected, internalization of $43 \mathrm{QH} t \mathrm{tt}$ resulted in greater impairment in the coordinated network activity, correlated with the toxic effects of the polyglutamine expansion $[280,437,438]$. A detailed understanding of the mechanisms of internalization of monomeric and aggregated forms of aSyn, Tau and $\mathrm{Htt}$ will be invaluable for the development of potential therapies for preventing the interneuronal transfer of proteins, without interfering with the physiological transfer of non-pathogenic forms.

Overall, our systematic study compares the transfer of disease-related proteins through various cellular mechanisms, and between different cell types. In particular, we emphasize that protein release, either in a free form or in EVs, induces diverse effects in neighbouring receptor cells, and that great care is important when considering the development of therapeutic strategies to avoid interfering with normal physiological intercellular communication.

\section{Acknowledgments}

We thank Prof. Dr. Ray Truant (McMaster University, Ontario, Canada) for kindly providing the Htt antibody used in the immunoblot experiments [anti-N17 (1-8)], to Prof. Dr. Hilal Lashuel for kindly providing the recombinant 23QHtt and 43QHtt (Lausanne, Switzerland), and to Prof. Dr. Flaviano Giorgini (Leicester University, Leicester, United Kingdom) that kindly provided the Htt lentiviral vectors. We thank Sabine König and Uwe Plessmann from Max Planck Institute for Biophysical Chemistry (Göttingen, Germany) and Christof Lenz from the Core Facility Proteomics at University Medical Center Göttingen (Göttingen, Germany) for assisting with mass spectrometry analysis. We thank Dr. Nicolás Lemus (Göttingen, Germany) for support with flow cytometry experiments. 


\section{Funding}

TFO is supported by European Union's Horizon 2020 research and innovation program under grant agreement No. 721802 (SynDegen), and by the Deutsche Forschungsgemeinschaft (DFG, German Research Foundation) under Germany's Excellence Strategy - EXC 2067/1- 390729940, by SFB1286 (B8). TG is supported by the European Research Council (ERC) under the European Union's Horizon 2020 research and innovation programme (grant agreement number 724822). This work was partly supported by the Göttingen Graduate School for Neurosciences, Biophysics, and Molecular Biosciences (DFG grant GSC 226/4).

\section{Author contributions}

T.F.O. and I.C.B. conceived the study. I.C.B performed all the cell culture, molecular biology, and imaging experiments. M.H.K and I.C.B established the spike sorting framework, performed MEA experiments and data analysis. E.V prepared the microglia samples for qPCR, imaging and the recombinant aSyn. W.M performed the immuno-EM experiments in the stable cell lines. D.R performed the EM experiments in EVs. E.G performed the cloning and prepared the lentiviral vectors. C.F. prepared the lentiviral vectors. I.P and H.U performed the mass spectrometry experiments. M.Z. provided the recombinant full-length 2N4R Tau. T.G. provided methodology and resources for the MEA experiments. I.C.B analysed and interpreted the data. I.C.B generated the graphs and figures. I.C.B. and T.F.O. wrote the manuscript. T.F.O. supervised the work.

\section{Declaration of Interests}

The authors declare no competing interests.

\section{Materials and Methods}

\section{Ethics statement}

Mice (C57BL6/J\#00245 background) were bred and maintained under specific pathogen free conditions in the animal facility of the University Medical Center Göttingen (Göttingen, Germany). All animal procedures were performed in accordance with the European Community (Directive 2010/63/EU), institutional and national guidelines (license numbers 19.3213 and T 20.7). 


\section{Primary cultures}

\section{Neuronal cultures}

C57BL6/J\#00245 wild-type E15.5 embryos from the animal facility of the University Medical Center Göttingen (Göttingen, Germany) were used for the preparation of primary cortical neuronal cultures, as previously described [534]. In detail, pregnant animals were anesthetized using carbon dioxide intoxication and the embryos extracted from the uterus. After removal of the meninges, cortex was dissected under a stereomicroscope and the tissue was transferred to ice-cold $1 x$ Hanks' balanced salt solution $\left(\mathrm{CaCl}_{2}\right.$ and $\mathrm{MgCl}_{2}$ free) (HBSS; Gibco Invitrogen, CA, USA) supplemented with $0.5 \%$ sodium bicarbonate solution (SigmaAldrich, MO, USA). Trypsinization was performed at $37^{\circ} \mathrm{C}$ for 15 minutes $(\mathrm{min})(100 \mu \mathrm{L}$ of $0.25 \%$ trypsin; Gibco Invitrogen, CA, USA), and the reaction was stopped with the addition of $100 \mu \mathrm{L}$ fetal bovine serum (FBS; Anprotec, Bruckberg, Germany) and 100 $\mathrm{L}$ DNase I (0.5 $\mathrm{mg} / \mathrm{mL}$; Roche, Basel, Switzerland). After dissociation, the cell suspension was centrifuged at $300 \times \mathrm{g}$ for $5 \mathrm{~min}$ and then resuspended in pre-warmed neurobasal medium (Gibco Invitrogen, CA, USA) supplemented with $2 \%$ B27 supplement (Gibco Invitrogen, CA, USA), 0.25\% GlutaMAX (Gibco Invitrogen, CA, USA) and 1\% penicillin- streptomycin (PAN Biotech, Aidenbach, Germany). Cells were seeded on coverslips coated with poly-L-ornithine (0.1 $\mathrm{mg} / \mathrm{mL}$ in borate buffer) (PLO; Sigma-Aldrich, MO, USA) or culture plates (Corning, Merck, Darmstadt, Germany) for immunocytochemistry and western blot experiments. Cells were maintained at $37^{\circ} \mathrm{C}$ with $5 \% \mathrm{CO}^{2}$, and fresh medium was added every $3-4$ days.

\section{Microglial cultures}

Primary microglia were obtained from mixed glial cell cultures from C57BL6/J\#00245 wild-type P0 pups from the animal facility of the University Medical Center Göttingen (Göttingen, Germany), as previously described [587]. Briefly, meninges were removed from the isolated brains and the tissue was collected into ice cold 1x HBSS (Gibco Invitrogen, CA, USA). The supernatant was removed, the brains were washed 3 times with HBSS solution (without $\mathrm{Ca}^{2+}, \mathrm{Mg}^{2+}$ and phenol) (PAN Biotech, Aidenbach, Germany) and incubated with $0.05 \%$ trypsin-EDTA (PAN Biotech, Aidenbach, Germany) in a water bath at $37^{\circ} \mathrm{C}$ during 10 min. Trypsin was aspirated and the digestion was stopped by adding $0.5 \mathrm{mg} / \mathrm{mL}$ DNase I (Roche, Basel, Switzerland) in microglia medium [Dulbecco's modified Eagle's medium (DMEM; PAN Biotech, Aidenbach, Germany) supplemented with 0.5\% penicillin- streptomycin (PAN Biotech, Aidenbach, Germany) and 10\% FBS (Anprotec, Bruckberg, Germany)]. Tissue was shortly incubated for $2-3 \mathrm{~min}$ at $37^{\circ} \mathrm{C}$ in the water bath and carefully homogenized into single-cell suspensions with a glass pipette. Suspension was centrifuged for $10 \mathrm{~min}$ at $800 \mathrm{xg}$, the supernatant was discarded, and the pellet was resuspended in microglia medium. Cell 
suspension was plated into T75 flask (Corning, Merck, Darmstadt, Germany) and incubated overnight at $37^{\circ} \mathrm{C}$ and $5 \% \mathrm{CO}^{2}$. On the following day, 2 days in vitro (DIV2), the cells were washed 3 times with pre-warmed HBSS solution (PAN Biotech, Aidenbach, Germany), 1 time with microglia medium and incubated with new medium until the next day. On DIV3, cell medium was replaced once more. On DIV5, the culture was stimulated by substitution of one third of the medium with L929 medium previously prepared in aliquots (see description in the cell lines section). The first harvest was performed on DIV8 by mild shaking and collection of the floating microglial cells. Medium was then centrifuged for $10 \mathrm{~min}$ at $800 \mathrm{xg}$, the pellet resuspended in microglia medium, and cells were plated into culture plates previously coated with PLO $(0.1 \mathrm{mg} / \mathrm{mL}$ in borate buffer) (Sigma-Aldrich, MO, USA) for immunocytochemistry, western blot, and gene expression studies. The culture was re-stimulated with new medium containing one third of L929 medium and incubated during 2-3 days until the next harvest. Three harvests were performed for the same culture.

\section{Astrocytic cultures}

Primary astrocytic cultures were prepared from C57BL6/J\#00245 wild-type cerebral cortices of PO pups from the animal facility of the University Medical Center Göttingen (Göttingen, Germany), as previously described [588]. After decapitation, meninges were removed, and the cortex was isolated from the brains and kept in ice cold 1x HBSS (Gibco Invitrogen, CA, USA). Tissue was digested in a fresh prepared solution of $0.25 \%$ trypsin (Gibco Invitrogen, CA, USA), $0.5 \mathrm{mg} / \mathrm{mL}$ DNase I (Roche, Basel, Switzerland), 1mM EDTA (Carl Roth, Karlsruhe, Germany), 10mM HEPES (Gibco Invitrogen, CA, USA), 2mg/mL bovine serum albumin (BSA) (Sigma-Aldrich, MO, USA) in $1 \times$ HBSS (Gibco Invitrogen, CA, USA) at $37^{\circ} \mathrm{C}$ for $20 \mathrm{~min}$. The reaction was stopped by adding astrocytes medium containing DMEM (PAN Biotech, Aidenbach, Germany) supplemented with 10\% FBS (Anprotec, Bruckberg, Germany), $1 \%$ penicillin- streptomycin (PAN Biotech, Aidenbach, Germany) and 25mM HEPES (Gibco Invitrogen, CA, USA), followed by a short centrifugation at $800 \times g$ during $2 \mathrm{~min}$. After aspiration of the supernatant, the tissue was dissociated with astrocytes medium until obtain a cell homogenate. After centrifugation at $800 \mathrm{xg}$ during $5 \mathrm{~min}$, the pellet was resuspended in medium, and cells were plated in a T75 $\mathrm{cm}^{2}$ flask (Corning, Merck, Darmstadt, Germany). Mixed culture was incubated at $37^{\circ} \mathrm{C}$ with $5 \% \mathrm{CO}^{2}$ for 3 days. At DIV1 cells were washed with warm HBSS solution (PAN Biotech, Aidenbach, Germany) and fresh media was added to the culture. The mixed astrocytic culture was agitated at DIV7 in an orbital plate shaker at $200 \mathrm{rpm}$ for $40 \mathrm{~min}$ at $37^{\circ} \mathrm{C}$. Cell culture agitation was repeated at DIV 9 for 2.5 hours (h) at $250 \mathrm{rpm}$ $\left(37^{\circ} \mathrm{C}\right)$. A third shake was performed on DIV10 at $350 \mathrm{rpm}$, overnight at $37^{\circ} \mathrm{C}$. After each agitation step, the supernatant was aspirated and the remaining cells in the flask were washed 
1 time with warm HBSS solution (PAN Biotech, Aidenbach, Germany) and fresh media was added to the cells. Cells were detached at DIV11 using 0.25\% trypsin (Gibco Invitrogen, CA, USA) at $37^{\circ} \mathrm{C}$ for $5 \mathrm{~min}$. Culture medium was added to stopped trypsin action, and the cell suspension was collected and centrifuged at $800 \mathrm{xg}$ for $5 \mathrm{~min}$. Cell pellet was resuspended in medium and cells were seeded with fresh medium on plates or coverslips (Corning, Merck, Darmstadt, Germany) coated with PLO $(0.1 \mathrm{mg} / \mathrm{mL}$ in borate buffer) (Sigma-Aldrich, MO, USA) for immunocytochemistry, western blot and gene expression studies at the appropriate density.

\section{Cell lines}

\section{Mouse fibroblast L929 cells}

Generation of L929-conditioned medium as performed as previously described [587]. L929 mouse fibroblast cells (kindly provided by Prof. Dr Hannelore Ehrenreich, MPI-EM, Göttingen, Germany) were plated into a $T 175 \mathrm{~cm}^{2}$ cell culture flask (Corning, Merck, Darmstadt, Germany) with $100 \mathrm{~mL}$ culture medium for 7 days containing DMEM (PAN Biotech, Aidenbach, Germany) supplemented with 10\% FBS (Anprotec, Bruckberg, Germany) and 1\% penicillinstreptomycin (PAN Biotech, Aidenbach, Germany) at $37^{\circ} \mathrm{C}$ with $5 \% \mathrm{CO}^{2}$. The media was then collected, sterilized by filtration with $0.22 \mu \mathrm{m}$ filter (Sartorius, Göttingen, Germany) and stored at $-20^{\circ} \mathrm{C}$. The aliquots were freshly thawed when used for stimulation of the microglial cell culture.

\section{Human embryonic kidney cells}

Human embryonic kidney (HEK) 293 cells (ATTC, VA, USA) were maintained in DMEM medium (PAN Biotech, Aidenbach, Germany) supplemented with 10\% FBS (Anprotec, Bruckberg, Germany) and 1\% penicillin- streptomycin (PAN Biotech, Aidenbach, Germany) at $37^{\circ} \mathrm{C}$ in a $5 \% \mathrm{CO}^{2}$ atmosphere.

\section{Transfection protocol}

HEK 293 cells were seeded in cell culture plates (Corning, Merck, Darmstadt, Germany) in the day before at the appropriate density. Transfection protocol was performed using Metafectene (Biotex, TX, USA) according to the manufacturer's instructions and using equimolar amounts of the plasmids of interest. The following plasmids used for the transfection protocol: pcDNA 3.1, pcDNA 3.1-aSyn, pcDNA 3.1-Tau (4R2N), pcDNA 3.1-22QHtt exon 1 (190, CAG, ID CHDI-90000027, Coriell Institute), pcDNA 3.1-72QHtt exon 1 (1-90, CAG, ID CHDI-90001882-1, Coriell Institute), mCitrine-USP19 (Plasmid \#78593, Addgene), mCitrine- 
USP19 C506S (Plasmid \#78594, Addgene). After 4h of transfection, fresh medium was added to the cells (as described above in the cell lines section). Cells were collected or stained for further western blot or immunocytochemistry analysis after $24 \mathrm{~h}$ or $72 \mathrm{~h}$ as described in each experiment section. USP19 plasmids were kindly provided by Prof. Dr. Yihong Ye through Addgene (Lee et al., 2016), and Htt plasmids were kindly provided by NIGMS Human Genetic Cell Repository at the Coriell Institute for Medical Research.

\section{Lentivirus production}

Production of FUGW-aSyn-EGFP, FUGW-EGFP-Tau, pRRL-CMV-25QHtt-EGFPPRE-SIN and pRRL-CMV-103QHtt-EGFP-PRE-SIN lentivirus was performed as previously described [535]. Htt lentiviral vectors were kindly provided by Prof. Dr. Flaviano Giorgini (Leicester University, Leicester, United Kingdom) [589]. HEK 293 cells were seeded in culture plates (Corning, Merck, Darmstadt, Germany) and incubated in DMEM (PAN Biotech, Aidenbach, Germany) supplemented with 10\% FBS (Anprotec, Bruckberg, Germany) and 1\% penicillin-streptomycin (PAN Biotech, Aidenbach, Germany) overnight at $37^{\circ} \mathrm{C}$ with $5 \% \mathrm{CO}^{2}$. On the following day, cells were incubated with DMEM with 2\% FBS (Anprotec, Bruckberg, Germany), transfection medium for $5 \mathrm{~h}$ before transfection. Cells were transfected using

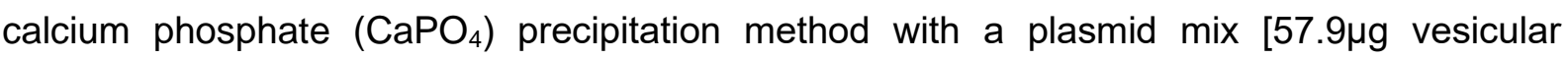
stomatitis virus glycoprotein (VSVG) packing virus, $144 \mu$ of Delta 8.9 packaging virus, and $160 \mu \mathrm{g}$ of the plasmid of interest]. The DNA mix was added to $6 \mathrm{~mL}$ of $1 \times$ BBS (50 mM BES, $\left.280 \mathrm{mM} \mathrm{NaCl}, 1.5 \mathrm{mM} \mathrm{Na}_{2} \mathrm{HPO}_{4}\right)$ and $0.36 \mathrm{~mL} \mathrm{CaCl} 2\left(2.5 \mathrm{M} \mathrm{CaCl}_{2}\right)$ was added to the mixture in a vortex shaker in the dark under sterile conditions. Before adding the mixture to the cells, solution was incubated $20 \mathrm{~min}$ in the dark. On the following day, cells were incubated with Panserin (PAN Biotech, Aidenbach, Germany) supplemented with $1 \%$ of non-essential amino acids (MEM, Gibco Invitrogen, CA, USA) and 1\% penicillin- streptomycin (PAN Biotech, Aidenbach, Germany). Viruses were harvested $72 \mathrm{~h}$ after transfection and centrifuged at $3000 \mathrm{xg}$ for $15 \mathrm{~min}$ at $4^{\circ} \mathrm{C}$. The supernatant was filtrated through a $0.45 \mu \mathrm{m}$ filter (Sartorius, Göttingen, Germany) and incubated with 1x PEG solution (SBI System Bioscience, CA, USA) to pellet the viruses. Viruses were spin down by centrifugation at $1500 \mathrm{xg}$ during $30 \mathrm{~min}\left(4^{\circ} \mathrm{C}\right)$ after 2 days of incubation at $4^{\circ} \mathrm{C}$. The pellet was resuspended in $100 \mu \mathrm{L}$ Panserin (PAN Biotech, Aidenbach, Germany).

\section{Lentiviral infections}

For neuronal cortical cultures, cells were infected at DIV14 with FUGW-aSyn-EGFP, FUGW-EGFP-Tau, pRRL-CMV-25QHtt-EGFP-PRE-SIN and pRRL-CMV-103QHtt-EGFP- 
PRE-SIN and collected for further analyses at DIV19. Culturing conditions were the same as specified above (primary culture section).

Stable cell lines expressing alpha-synuclein (aSyn), Tau and huntingtin ( $\mathrm{Htt}$ ) were established by lentiviral infection of HEK 293 cells with FUGW-aSyn-EGFP, FUGW-EGFPTau, pRRL-CMV-25QHtt-EGFP-PRE-SIN and pRRL-CMV-103QHtt-EGFP-PRE-SIN. Cells were incubated during 5 days with the viruses and after 3 passages the infection rate was confirmed by microscopy (more than $90 \%$ of positive cells).

\section{Lactate dehydrogenase assay}

Cytotoxicity in the cell cultures (primary cortical neurons and cell lines) was assessed using the cytotoxicity lactate dehydrogenase $(\mathrm{LDH})$ detection kit according to the manufacturer's instructions (Roche, Basel, Switzerland). Furthermore, culture medium was centrifuged at $500 \mathrm{xg}$ for $5 \mathrm{~min}$ to pellet cell debris before used in the experiments.

\section{Immunocytochemistry experiments}

Primary cortical neurons and cell lines cultures after the treatments were washed with 1x PBS (PAN Biotech, Aidenbach, Germany) and fixed with $4 \%$ of paraformaldehyde solution (PFA) for $20 \mathrm{~min}$ (home-made) at room temperature (RT). PFA autofluorescence was quenched by incubation with $50 \mathrm{mM}$ of ammonium chloride $\left(\mathrm{NH}_{4} \mathrm{Cl}\right)$ solution for $30 \mathrm{~min}$ at RT. Cells were permeabilized with $0.1 \%$ Triton X-100 (Sigma-Aldrich, MO, USA) for 10 min at RT, and then incubated with $2 \%$ BSA in PBS (NZYTech) blocking solution for $1 \mathrm{~h}$ at RT. Incubation with primary antibody was performed overnight at $4^{\circ} \mathrm{C}$ [aSyn $(1: 1000,610787, \mathrm{BD}$ Biosciences), aSyn (phospho S129) (1:500, ab51253, Abcam), $\alpha$-tubulin (1:1000, 302217, Synaptic Systems), Glial Fibrillary Acidic Protein (GFAP) (1:1000, Z0334, Dako), Htt (1:500, MAB5374, Merck Millipore), Iba1 (1:1000, ab5076, Abcam), LC3 (1:500, PM036, MBL International), MAP2 (1:1000, ab11267, Abcam), Synaptophysin (1:500, 101002, Synaptic Systems), Tau (1:1000, MN1000, Thermo Fisher Scientific)]. Subsequently, the cells were washed with 1x PBS (PAN Biotech, Aidenbach, Germany) and then incubated with fluorescence conjugated secondary antibodies for $2 \mathrm{~h}$ at RT [Alexa Fluor 488 donkey (1:1000, A21206, A21202, A11055, A21208, Invitrogen), Alexa Fluor 555 donkey (1:1000, A31572, A31570, A21432, Invitrogen), Alexa Fluor 633 goat (1:1000, A21050, Invitrogen), Alexa Fluor 633 donkey (1:1000, A21082, Invitrogen) and Alexa Fluor 680 donkey (1:1000, A10043, Invitrogen)]. Lastly, nuclei were counter-stained with DAPI (Carl Roth, Karlsruhe, Germany) and mounted with mowiol (home-made) for imaging experiments. 


\section{Western blots}

Primary cortical neurons and cell lines cultures after the treatments were washed with 1x PBS (PAN Biotech, Aidenbach, Germany) and lysed in RIPA buffer [50mM Tris, pH 8.0, $0.15 \mathrm{M} \mathrm{NaCl}, 0.1 \%$ SDS, $1.0 \%$ NP-40, 0.5\% Na-Deoxycholate, 2mM EDTA, supplemented with protease and phosphatase inhibitors cocktail [(cOmplete ${ }^{\mathrm{TM}}$ protease inhibitor and PhosSTOP ${ }^{\mathrm{TM}}$ phosphatase inhibitor) (Roche, Basel, Switzerland)]. Lysate protein concentrations were determined using the Bradford protein assay (Bio-Rad, CA, USA). Samples containing $30 \mu \mathrm{g}$ of protein were denaturated for $5 \mathrm{~min}$ at $95^{\circ} \mathrm{C}$, loaded into $12 \%$ SDSPAGE gels and transferred to nitrocellulose membranes using iBlot 2 (Invitrogen, CA, USA). Membranes were incubated in blocking solution containing 5\% skim milk (Sigma-Aldrich, MO, USA) in tris-buffered saline ( $\mathrm{pH} 8$ ) with $0.05 \%$ tween 20 (TBS-T). Primary antibody incubation was performed overnight at $4^{\circ} \mathrm{C}$ diluted in $5 \%$ BSA (Sigma-Aldrich, MO, USA) in TBS-T. The following primary antibodies were used in this study: Alix (1:1000, ab117600, Abcam), aSyn (1:3000, 610787, BD Biosciences), aSyn (phospho S129) (1:1000, ab51253, Abcam), $\alpha-$ tubulin (1:1000, 302217, Synaptic Systems), $\beta$-Actin (1:10000, A5441, Sigma-Aldrich), Annexin-A2 (1:1000, ab178677, Abcam), APG5L/ATG5 (1:1000, ab23722, Abcam), Flotilin-1 (1:1000, \#18634, Cell Signaling), GFP (1:1000, 11814460001, Roche), Glial Fibrillary Acidic Protein (GFAP) (1:1000, Z0334, Dako), Huntingtin anti-N17 (1-8) [1:5000, kindly provided by Prof. Dr. Ray Truant (McMaster University, Ontario, Canada)], iNOS / NOS II (1:1000, 06-573, Upstate), Iba1 (1:1000, ab178846, Abcam), LC3 (1:1000, PM036, MBL International), MAP2 (1:1000, ab11267, Abcam), p62/SQSTM1 (1:1000, ab91526, Abcam), Synaptophysin (1:1000, 101002, Synaptic Systems), Tau (1:5000, A0024, Dako), USP19 (1:1000, A301-587A-M, Biomol). On the next day, the membranes were washed with TBS-T and incubated for $2 \mathrm{~h}$ with horseradish peroxidase (HRP) conjugated secondary antibodies [ECL ${ }^{\mathrm{TM}}$ Rabbit or Mouse IgG, HRP-linked whole antibody (1:10000, NA934V or NXA931, Amersham)]. Subsequently, membranes were washed with TBS-T and incubated with a chemiluminescent HRP substrate (Millipore, MA, USA) for bands visualization in a chemiluminescence system (Fusion FX Vilber Lourmat, Vilber, France). Intensities of specific bands were normalized to a protein loading control or to the total protein levels marked using MemCode ${ }^{\mathrm{TM}}$ Reversible Protein (Thermo Fisher Scientific, MA, USA).

\section{Native-PAGE}

For native gel electrophoresis, samples were mixed with protein sample buffer $(0.31 \mathrm{M}$ Tris $\mathrm{HCl} \mathrm{pH} \mathrm{6.8,50 \%} \mathrm{Glycerol,} \mathrm{0.4 \%} \mathrm{Bromophenol} \mathrm{blue).} \mathrm{Samples} \mathrm{were} \mathrm{loaded} \mathrm{on} \mathrm{pre-cast}$ vertical Serva gels 4-16\% (Serva, Heidelberg, Germany) and ran according to the manufacturer's instructions using the appropriate buffers $[50 \mathrm{mM}$ BisTris- $\mathrm{HCl} \mathrm{pH} 7.0$ for the 
anode buffer; $50 \mathrm{mM}$ Tricin, $15 \mathrm{mM}$ BisTris- $\mathrm{HCl} \mathrm{pH} 7.0$ for the cathode buffer] (Serva, Heidelberg, Germany). Proteins were transferred to nitrocellulose membranes using iBlot 2 (Invitrogen) and immunoblotting was performed as previously described in the western blot section.

\section{Isolation of extracellular vesicles}

Isolation of ectosomes and exosomes was performed as previously described [562]. HEK cells expressing the proteins of interest were grown in conditioned medium (depleted of FBS-derived exosomes). Briefly, DMEM (PAN Biotech, Aidenbach, Germany) supplemented with $20 \%$ FBS (Anprotec, Bruckberg, Germany) and $2 \%$ penicillin- streptomycin (PAN Biotech, Aidenbach, Germany) was centrifuged in polypropylene tubes (Optiseal; Beckman Coulter, CA, USA) in a fixed rotor (type $70 \mathrm{Ti}$, Beckman) during $16 \mathrm{~h}$ at $100000 \times \mathrm{xg}\left(4^{\circ} \mathrm{C}\right)$, as previously described [497]. The medium was subsequently diluted with DMEM medium (PAN Biotech, Aidenbach, Germany) for a final concentration of $10 \%$ FBS (Anprotec, Bruckberg, Germany) and $1 \%$ penicillin- streptomycin (PAN Biotech, Aidenbach, Germany). Cells were seeded in T75 $\mathrm{cm}^{2}$ flasks (Corning, Merck, Darmstadt, Germany) and incubated with fresh conditioned media during $24 \mathrm{~h}$ at $80 \%$ confluency. The media was collected, and protease and phosphatase inhibitors [(cOmplete ${ }^{\mathrm{TM}}$ protease inhibitor and PhosSTOP ${ }^{\mathrm{TM}}$ phosphatase inhibitor) (Roche, Basel, Switzerland)] were added before centrifuging for $10 \mathrm{~min}$ at $300 \mathrm{xg}\left(4^{\circ} \mathrm{C}\right)$. A second centrifugation was performed at $2000 \times \mathrm{g}$ for $20 \mathrm{~min}\left(4^{\circ} \mathrm{C}\right)$. Supernatant was transferred into ultra-clear tubes (Beckman Coulter, CA, USA) and ultracentrifuged in a swing rotor (TH-641 Sorvall; Thermo Fisher Scientific, MA, USA) during 90 min at $20000 \times g\left(4^{\circ} \mathrm{C}\right)$. Supernatant was carefully transferred into a new centrifuge tube and the pellet containing ectosomes was resuspended in ice cold PBS with protease and phosphatase inhibitors (PAN Biotech, Aidenbach, Germany). The medium was centrifuged in a swing rotor (TH-641 Sorvall; Thermo Fisher Scientific, MA, USA) during $90 \mathrm{~min}$ at $100000 \mathrm{xg}\left(4^{\circ} \mathrm{C}\right)$ to purify exosomes. The pellet was resuspended in ice cold PBS with protease and phosphatase inhibitors (PAN Biotech, Aidenbach, Germany). Ectosomal and exosomal pellets were diluted in PBS (PAN Biotech, Aidenbach, Germany) and centrifuged at the correspondent velocities to wash and concentrate the vesicles fractions. The pellets were resuspended in 100 $\mu$ l of ice cold 1x PBS with protease and phosphatase inhibitors (PAN Biotech, Aidenbach, Germany). Protein concentrations were determined by the BCA assay following the manufacturer's instructions (Thermo Fisher Scientific, MA, USA). 


\section{Labelling of extracellular vesicles}

Extracellular vesicles were labelled with a fluorescent dye following a protocol previously described [521, 562]. Briefly, extracellular vesicles $(60-100 \mu \mathrm{g}$ of total protein in $50 \mu \mathrm{L}$ PBS) were incubated with Alexa Fluor 633 C5-maleimide dye $(200 \mu \mathrm{g} / \mathrm{mL}$; Invitrogen, Carlsbad, California, CA, USA) for $1 \mathrm{~h}$ in the dark at RT. Samples were resuspended in $10 \mathrm{~mL}$ PBS (PAN Biotech, Aidenbach, Germany) and centrifugated during $90 \mathrm{~min}$ at $20000 \times \mathrm{xg}\left(4^{\circ} \mathrm{C}\right)$ for ectosomes and 100000xg for 90 min for exosomes $\left(4^{\circ} \mathrm{C}\right)$ in a swing rotor (TH-641 Sorvall; Thermo Fisher Scientific, MA, USA). As a control, PBS was similarly incubated with the dye to confirm the removal of the dye excess.

\section{NTA analysis}

Extracellular vesicles size distribution and particle number were measured using nanoparticle tracking analysis (NTA) with NanoSight LM10 instrument (LM14 viewing unit equipped with a $532 \mathrm{~nm}$ laser) (NanoSight, Salisbury, UK). For each replicate, ectosomes and exosomes fractions were diluted in 1x PBS (PAN Biotech, Aidenbach, Germany) to a final volume of $400 \mathrm{~mL}$ prior to analysis, according to the manufacturer's recommendations (NanoSight, Salisbury, UK). Measurements were recorded using NTA 2.3 software with a detection threshold of 5 , captured with a camera level of 16 at $25^{\circ} \mathrm{C}$, in videos of $5 \times 60 \mathrm{~s}$ repeated 4 times.

\section{Electron microscopy (EM)}

Electron microscopy (EM) images from extracellular vesicles was performed following a protocol previously described [562]. Isolated ectosomes and exosomes were bound to a glow discharged carbon foil covered grids. Samples were sained with $1 \%$ uranyl acetate (aq.) and then evaluated at RT using Talos L120C transmission electron microscope (Thermo Fisher Scientific, MA, USA).

\section{Immuno-EM}

Immuno-EM images from HEK 293 cells expressing FUGW-aSyn-EGFP, FUGWEGFP-Tau, pRRL-CMV-25QHtt-EGFP-PRE-SIN and pRRL-CMV-103QHtt-EGFP-PRE-SIN were performed following a protocol previously described [590]. Briefly, cells were fixed with $4 \%$ formaldehyde and $0.2 \%$ glutaraldehyde in $0.1 \mathrm{M}$ phosphate buffer. The cells were washed and then scraped from the dish in $0.1 \mathrm{M}$ phosphate buffer containing $1 \%$ gelatine, spun down, and resuspended in $10 \%$ gelatine in $0.1 \mathrm{M}$ phosphate buffer at $37^{\circ} \mathrm{C}$. After spinning down again, the resulting pellets in gelatine were cooled on ice, removed from the tubes, and cut in small 
blocks. These blocks were infiltrated in 2.3M sucrose in $0.1 \mathrm{M}$ phosphate buffer, mounted onto aluminium pins for cryo-ultramicrotomy (Leica Microsystems, Vienna, Austria) and frozen in liquid nitrogen. Ultrathin cryosections were cut with a cryo-immuno diamond knife (Diatome, Biel, Switzerland) using a UC6 cryo-ultramicrotome (Leica Microsystems, Vienna, Austria) and picked up in a $1: 1$ mixture of $2 \%$ methylcellulose and $2.3 \mathrm{M}$ sucrose. For immuno-labelling, sections were incubated with 3H9 GFP primary antibody (\#029762, ChromoTek, PlaneggMartinsried, Germany), followed by the secondary antibody (\#112-4102, Rockland Immunochemicals, PA, USA) and then protein A-gold (10nm) (Cell Microscopy Core, Utrecht, Netherlands). Sections were analysed with a LEO EM912 Omega (Zeiss, Oberkochen, Germany) and digital micrographs were obtained with an on-axis 2048x2048-CCD camera (TRS).

\section{Proteomic analyses of extracellular vesicles}

For the proteomic analyses, ectosomes and exosomes samples were resuspended in Laemmli sample buffer and separated in SDS-PAGE gel, as previously described [562]. Briefly, the entire lane was cut in 23 gel pieces and tryptically digested [539]. Extracted peptides were analysed in technical replicates by liquid chromatography coupled to mass spectrometry (LCMS) on a Dionex UltiMate 3000 RSLCnano system (Thermo Fisher Scientific, Waltham, USA) connected to an Orbitrap Fusion mass spectrometer (Thermo Fisher Scientific, Waltham, USA). A 43 min gradient ranging from $8 \%$ to $37 \%$ acetonitrile on an in-house packed $\mathrm{C} 18$ column was used to separate the peptides $(75 \mu \mathrm{m}$ x $30 \mathrm{~cm}$, Reprosil-Pur 120C18-AQ, $1.9 \mu \mathrm{m}$, Dr. Maisch $\mathrm{GmbH}$, Ammerbuch, Germany) at $300 \mathrm{nl} / \mathrm{min}$ flow rate. MS1 spectra were acquired with 120000 resolution (full width at half maximum, FWHM) and a scan range from 350 to 1600 $\mathrm{m} / \mathrm{z}$. Within a cycle time of $3 \mathrm{~s}$, precursor ions with a charge state between +2 and +7 were selected individually with a $1.6 \mathrm{~m} / \mathrm{z}$ isolation window and were fragmented with a normalized collision energy of 35 by higher energy collisional dissociation (HCD). MS2 spectra were acquired in the ion trap with $20 \%$ normalized AGC and dynamic injection time. Once selected precursors were excluded from another fragmentation event for $30 \mathrm{~s}$. Raw acquisition files were subjected to database search with Maxquant (version 1.6.2.10) [540] against the reference proteome of Homo sapiens (downloaded on 02/19/2019) and the GFP fusion proteins. Default settings were used unless stated differently below. Fractions were defined according to the cutting of the gel lanes and experiments were defined on the level of technical replicates. Unique and razor peptides were used for label-free quantification except for the GFP fusion constructs where all peptides were used due to the difficulty of peptide assignment and protein grouping related to the EGFP tag. 
All the proteomic data analysis were performed with Perseus (version and 1.6.15.0) [541]. The initial data was filtered for reverse hits, potential contaminants and hits only identified by site. Quantitative values were averaged across technical replicates ignoring missing values. A two-sample t-test was performed on biological replicates of the samples with an artificial within groups variance (s0) of 0.1 and a permutation-based FDR of 0.1 for multiple testing correction. These results were visualized in volcano plots.

\section{Secretion and internalization assays}

The collection of the cell media and evaluation of proteins secretion was performed using an adapted protocol from a previous study [455]. Briefly, $1 \mathrm{~mL}$ of medium from HEK cells expressing FUGW-aSyn-EGFP, FUGW-EGFP-Tau, pRRL-CMV-25QHtt-EGFP-PRE-SIN or pRRL-CMV-103QHtt-EGFP-PRE-SIN was collected after $24 \mathrm{~h}$ and centrifuged for $5 \mathrm{~min}$ at $500 \times g$ at $4{ }^{\circ} \mathrm{C}$ to pellet cell debris. Supernatants were concentrated 10 times in an Amicon ultra $10 \mathrm{~K}$ centrifugal filters (Millipore, MA, USA) following the manufacturer's instructions. Only $30 \mu \mathrm{L}$ of the $100 \mu \mathrm{L}$ final volume was analysed by western blot.

For the neuronal cell media, the DotBlot system was used to exclude the possibility that the proteins of interest could be retained in the centrifugal filters and change the results observed in the experiments. Briefly, $1 \mathrm{~mL}$ of the neuronal cell media was collected from primary cortical neurons expressing FUGW-aSyn-EGFP, FUGW-EGFP-Tau, pRRL-CMV25QHtt-EGFP-PRE-SIN or pRRL-CMV-103QHtt-EGFP-PRE-SIN and centrifuged for 5 min at $500 \times g\left(4^{\circ} \mathrm{C}\right)$. Only $300 \mu \mathrm{L}$ of the $1 \mathrm{~mL}$ final volume was directly applied into the system in a nitrocellulose membrane (Bio-Rad, CA, USA).

For the internalization experiments, $1 \mathrm{~mL}$ of medium from HEK cells expressing FUGWaSyn-EGFP, FUGW-EGFP-Tau, pRRL-CMV-25QHtt-EGFP-PRE-SIN or pRRL-CMV103QHtt-EGFP-PRE-SIN was collected after $24 \mathrm{~h}$ and centrifuged for $5 \mathrm{~min}$ at $500 \times \mathrm{g}\left(4^{\circ} \mathrm{C}\right)$. Afterwards, the media was added to naïve HEK cells for $72 \mathrm{~h}$.

\section{Flow cytometry experiments}

As described in the previous section, naïve HEK cells were incubated during $72 \mathrm{~h}$ with $1 \mathrm{~mL}$ of cell media from HEK cells expressing FUGW-aSyn-EGFP, FUGW-EGFP-Tau, pRRLCMV-25QHtt-EGFP-PRE-SIN or pRRL-CMV-103QHtt-EGFP-PRE-SIN. Subsequently, HEK cells were washed with 1x PBS and trypsinized (PAN Biotech, Aidenbach, Germany). Cell suspension was centrifuged during $5 \mathrm{~min}$ at $300 \mathrm{xg}\left(4^{\circ} \mathrm{C}\right)$. Cell pellet was resuspended in $1 \mathrm{x}$ PBS (PAN Biotech, Aidenbach, Germany) to remove residual cell media and centrifuged for 5 $\min$ at $300 \times g\left(4^{\circ} \mathrm{C}\right)$. The supernatant was removed by aspiration and the cell pellet was 
resuspended in $1 \mathrm{~mL}$ of ice cold 1x PBS (PAN Biotech, Aidenbach, Germany) with $0.1 \%$ of propidium iodide (Sigma-Aldrich, MO, USA). Cells without EGFP expression, treated with $0.1 \%$ triton-X (Sigma-Aldrich, MO, USA) or PBS alone were used as negative controls. 10000 events were acquired on a FACSAria II flow cytometer (BD Biosciences, NJ, USA). Flow cytometric data were analysed with FlowJo Analysis Software (BD Biosciences, NJ, USA).

\section{Treatment of cells with extracellular vesicles}

Primary cortical neurons and astrocytes were treated with $20 \mu \mathrm{g} / \mathrm{mL}$ of ectosomes or exosomes resuspended in 1x PBS (PAN Biotech, Aidenbach, Germany) with protease and phosphatase inhibitors [(cOmplete ${ }^{\mathrm{TM}}$ protease inhibitor and PhosSTOP ${ }^{\mathrm{TM}}$ phosphatase inhibitor) (Roche, Basel, Switzerland)]. Due to high toxicity, microglial cells were treated only with $10 \mu \mathrm{g} / \mathrm{mL}$ of extracellular vesicles. Treatment was performed in cortical neurons at DIV14, and cells were fixed or collected for further analyses at DIV15 (24h treatment). Microglial and astrocytic cell cultures were treated in the day after their plating for $24 \mathrm{~h}$, and then fixed or collected for further analyses. Pro-inflammatory stimulation was evaluated by the exposition to lipopolysaccharide (LPS; Thermo Fisher Scientific, MA, USA). Microglia cells were treated with $50 \mathrm{ng} / \mathrm{mL}$ and astrocytes with $100 \mathrm{ng} / \mathrm{mL}$ of LPS (Thermo Fisher Scientific, MA, USA). Culturing conditions were the same as specified above in the primary culture section.

\section{Gene expression studies - RNA isolation and Quantitative real-time PCR}

Total RNA was extracted from microglial and astrocytic cultures $24 \mathrm{~h}$ after treatment using TRIzol Reagent according to the manufacturer's instructions (Invitrogen, CA, USA). Reverse transcription of RNA to produce cDNA was performed using QuantiTect Reverse Transcription kit (Qiagen, MD, USA) following the protocol provided by the manufacturers. Quantitative real-time PCR (qPCR) analysis was performed on an Applied Biosystems Real-Time PCR Systems using SYBR Green Master Mix (Qiagen, MD, USA). The thermal cycler conditions were as following: $95^{\circ} \mathrm{C}$ for $10 \mathrm{~min}$, then 40 cycles at $95^{\circ} \mathrm{C}$ for $15 \mathrm{~s}$ and $60^{\circ} \mathrm{C}$ for $25 \mathrm{~s}$. Primers used in the quantitative real-time PCR experiments to evaluate the inflammatory markers were (mouse, sequence $5^{\prime}$ to $3^{\prime}$ ): IL-6 Forward- ATCCAGTTGCCTTCTTGGGACTGA, IL-6 ReverseTAAGCCTCCGACTTGTGAAGTGGT, IL-10 Forward- GGTTGCCAAGCCTTATCGGA, IL-10 Reverse- CACTCTTCACCTGCTCCACT, IL-1 $\beta$ Forward- TCATTGTGGCTGTGGAGAAG, IL-1 $\beta$ Reverse - AGGCCACAGGTATTTTGTCG, TNFa Forward- CCCTCTCATCAGTTCTATGG, TNFa Reverse- $\quad$ GGAGTAGACAAGGTACAACC, $\beta$-actin ForwardGCGAGAAGATGACCCAGATC and $\beta$-actin Reverse- CCAGTGGTACGGCCAGAGG. Fold change expressions were calculated using the $2^{-\triangle \triangle C T}$ method, with $\beta$-actin as a reference gene 
[591]. Quantification in the graphs show the normalized relative quantity (NRQ) values compared with the control.

\section{Treatment with recombinant monomeric protein}

Primary cortical neurons were treated at DIV14 with recombinant monomeric protein until DIV19 (5 days). Final concentration of protein in the cultures was 100nM and PBS was employed as negative control. Recombinant aSyn, full-length 2N4R Tau, 23QHtt and 43QHtt were prepared as previously described [592-594]. Culturing conditions were the same as specified above (primary culture section).

\section{Multielectrode array experiments}

Multi-electrode array (MEA) experiments were performed following standard protocols, as previously described $[543,562]$. Primary cortical neuronal cultures were plated directly on 60MEA200/30iR-Ti-gr planar arrays (60 electrodes, 30 $\mu \mathrm{m}$ electrode diameter, $200 \mu \mathrm{m}$ electrode spacing) (MultiChannel Systems, Reutlingen, Germany). The arrays were coated with poly-L-lysine overnight at $4^{\circ} \mathrm{C}(500 \mu \mathrm{g} / \mathrm{mL}$ in borate buffer; PLL) (Sigma-Aldrich, MO, USA) and the next day with laminin for $1 \mathrm{~h}$ at $\mathrm{RT}(5 \mu \mathrm{g} / \mathrm{mL}$ in distilled water) (Sigma-Aldrich, MO, USA). Neuronal cells were plated directly on top of the electrodes and treated with EVs or recombinant proteins. For the EVs recordings, cells were incubated with $20 \mu \mathrm{g} / \mathrm{mL}$ of ectosomes or exosomes containing the different disease-related proteins at DIV14 and recorded at DIV15, 24h after the treatment. In the recombinant monomeric protein experiments, cells were incubated with 100nM of aSyn, full Tau, 23QHtt exon 1 or 43QHtt exon 1 at DIV14 and recorded at DIV19, 5 days after the treatment. The neuronal activity was recorded using the MultiChannel MEA2100 system (MultiChannel Systems, Reutlingen, Germany) with temperature maintained at $35-37^{\circ} \mathrm{C}$. Recordings started 10 min after translocation of the arrays from the incubator to the recording stage to avoid movementinduced artifacts, and the spontaneous activity was recorded for 5-10 min. The electrode signals were amplified, band-pass filtered $(200 \mathrm{~Hz}$ to $3 \mathrm{kHz})$ and recorded digitally at $25 \mathrm{kHz}$, using the MultiChannel Experimenter software (version 2.17.7.0) (MultiChannel Systems, Reutlingen, Germany).

Spike sorting was carried out using a modified version of the Kilosort automatic sorting software, as previously described [544, 545, 562] (available at: https://github.com/MouseLand/Kilosort and https://github.com/dimokaramanlis/KiloSortMEA). Kilosort output was visually inspected and manually curated with the Phy2 software (https://github.com/cortex-lab/phy). Only spike clusters ("units") with a well-separated spike 
waveform and a clear refractory period were included in the data analysis and considered as originated from individual neuronal cells. The spike clusters were pre-processed and analysed using custom-made MATLAB scripts (Version: 9.7.0, R2019b; Mathworks, MA, USA). The raster plots, voltage traces, average firing rate and spike amplitude were measured from the spontaneous activity of each recorded cell. In all the recordings, we observed the occurrence of frequent bursts [groups of spikes occurring rapidly and consecutively with short inter-spike intervals, less than few tens of milliseconds (ms)], followed by quiescent periods longer than normal inter-spike intervals [generally several seconds (s) in our recordings]. The burst activity usually occurred synchronously for multiple cells over the array and in our analysis, and we focused on this population-wide synchronized bursts for further data analysis. To detect the concurrent bursts, the population firing rate was computed as a histogram (100ms bin size) of array-wide spiking activity. The peaks of the firing rate histogram were used to detect synchronous, array-wide bursts with at least 500 ms distance between two consecutive peaks. The peaks that were smaller than $1 / 5$ of the largest peak were excluded as they do not correspond to array-wide synchronous activity. A time window of $650 \mathrm{~ms}$ around each peak (150 ms before to $500 \mathrm{~ms}$ after) was defined as the burst window (onset and offset of each burst). For each recorded cell, the spikes belonging to bursts were measured during the defined burst windows, and cells with fewer than six spikes across all their detected bursts were excluded from this analysis. From the detected bursts, the following parameters were calculated: (1) burst rate of the culture as the number of bursts per time over the duration of each recording; (2) inter-burst-interval as the time between the measured offset of a burst and the onset of the following burst, calculated for each pair of successive bursts in a recording; (3) burst duration for each cell as the time between the cell's first and last spike during the burst window; (4) intra-burst-frequency as the rate of spikes occurring within a burst, averaged over all the detected bursts for each cell; and (5) percentage of spikes in bursts as the ratio of spikes occurring during bursts relative to the total number of spikes for each cell.

\section{Confocal microscopy imaging}

Imaging was performed on a Leica SP5 confocal laser scanning microscope equipped with hybrid detectors using Application Suite $X$ software with 100x immersion objective lenses (Leica Biosystems, Wetzlar, Germany). Samples were excited using 405 Diode, argon and helium-neon 633 lasers, pinhole $=1,0.2 \mu \mathrm{m}$ thickness $Z$ stacks and 2 averaging line-by-line. The acquisition settings were optimized to avoid underexposure and oversaturation effects and kept equal throughout image acquisition of the samples. 


\section{Quantifications and Statistical Analyses}

Analyses of the images was performed using ImageJ software (National Institutes of Health) [546]. The EVs uptake in neuronal cells was measured by the ration of the EVs signal area and cell area from different isolated areas chosen randomly within regions containing EVs signal. All data are presented as mean \pm SD. Data from at least three independent experiments and each replicate represents one independent experiment. To assess differences between two groups, two-tailed unpaired student t-test was performed using GraphPad Prism 9 software (GraphPad, CA, USA). To assess differences between more than two groups, significant differences were assessed by one-way ANOVA followed by multiple comparisons with significance between groups corrected by Bonferroni procedure using GraphPad Prism 9 software (GraphPad, CA, USA). Differences were considered to be significant for values of $p<0.05$ and are expressed as mean \pm SD. For mass spectrometry, the spectral count differences between samples were considered to be significant for FDR values $<0.1$ (see proteomic analyses section). 


\section{Supplementary Figures}

A

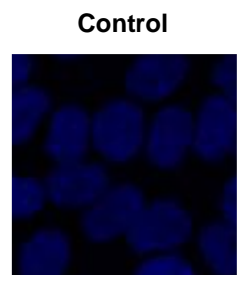

22QHtt

72QHtt

aSyn

Tau
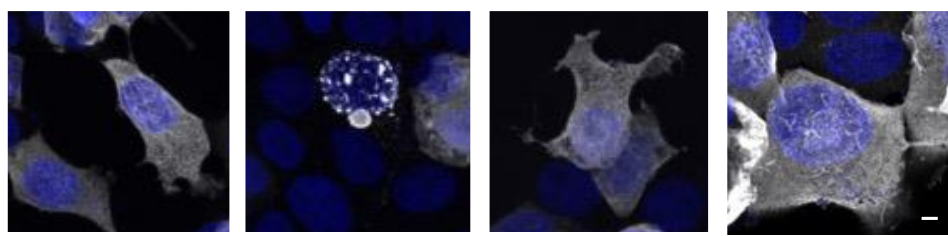

B
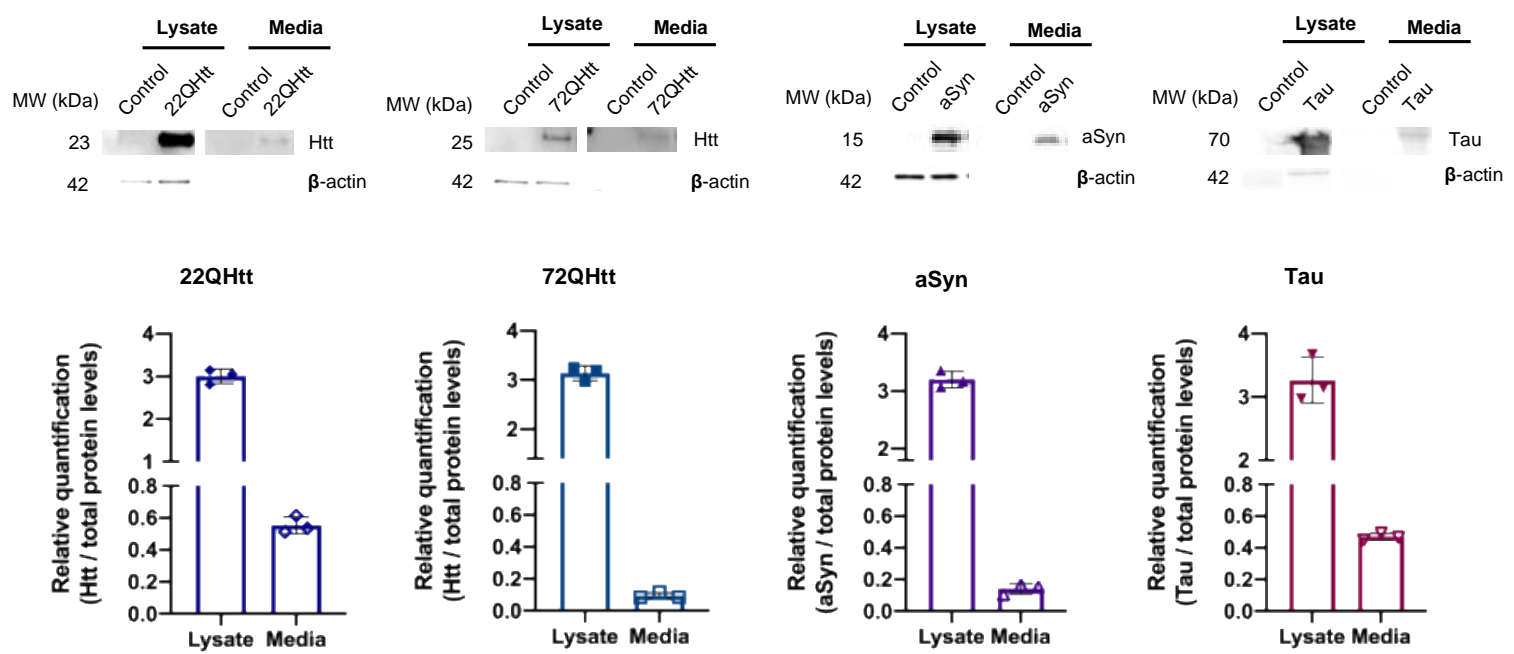

C

D
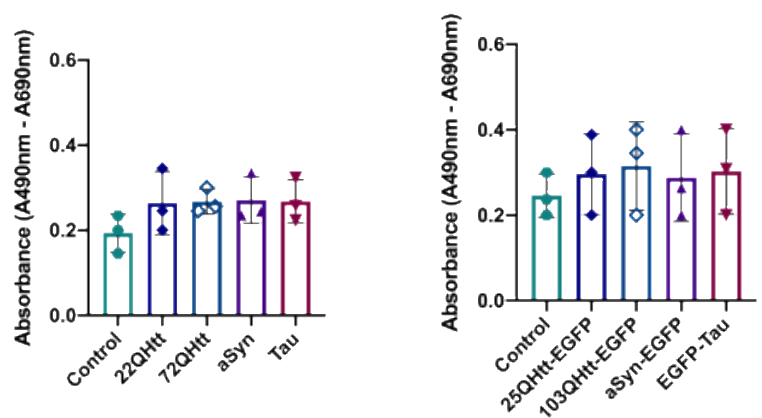

E

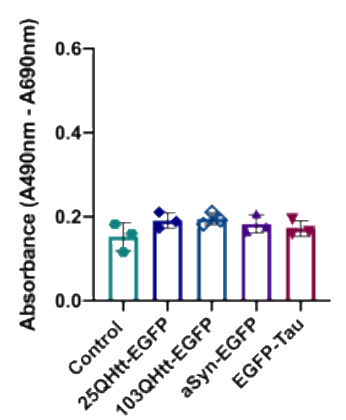

Supplementary Figure 1. Different disease-related proteins are secreted to the extracellular space. (A) Representative images of HEK cells transfected with plasmids encoding untagged $22 \mathrm{QHtt}, 72 \mathrm{QHtt}$, aSyn or Tau. Control cells were transfected with an empty plasmid. Scale bar $5 \mu \mathrm{m}$. (B) Western blots showing the protein levels in the lysates and released to the cell media of the different cells. Quantifications were normalized to total protein levels using MemCode. (C-E) LDH measurements confirm the absence of cell toxicity and cell death in (C) cells transfected with disease-relate proteins without tag, (D) in HEK cells stably expressing 25QHtt-EGFP, 103QHttEGFP, aSyn-EGFP or EGFP-Tau, and (E) in primary cortical neurons expressing 25QHtt-EGFP, 103QHtt-EGFP, aSyn-EGFP or EGFP-Tau. Data from at least three independent experiments for each condition. Data from at least three independent experiments for each condition. Significant differences were assessed by one-way ANOVA followed by multiple comparisons with significance between groups corrected by Bonferroni procedure. 
A
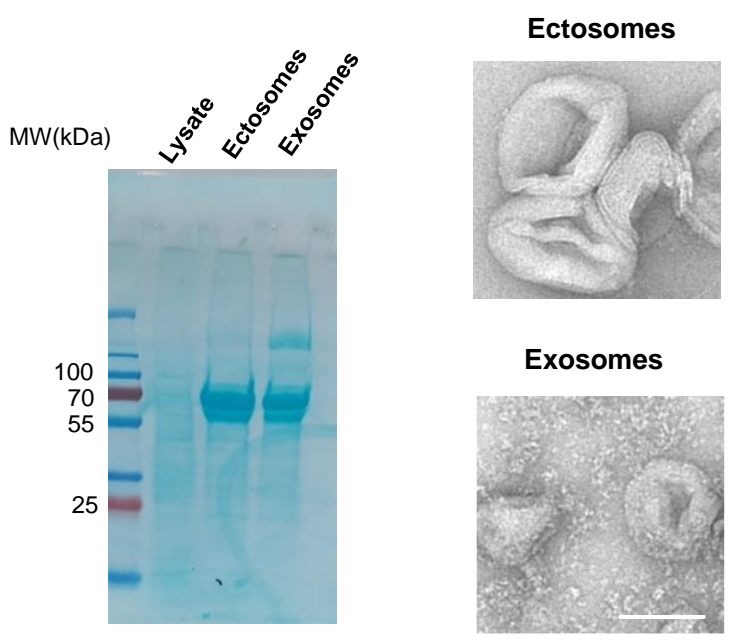

Exosomes

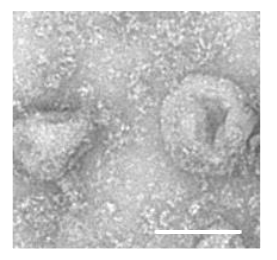

C

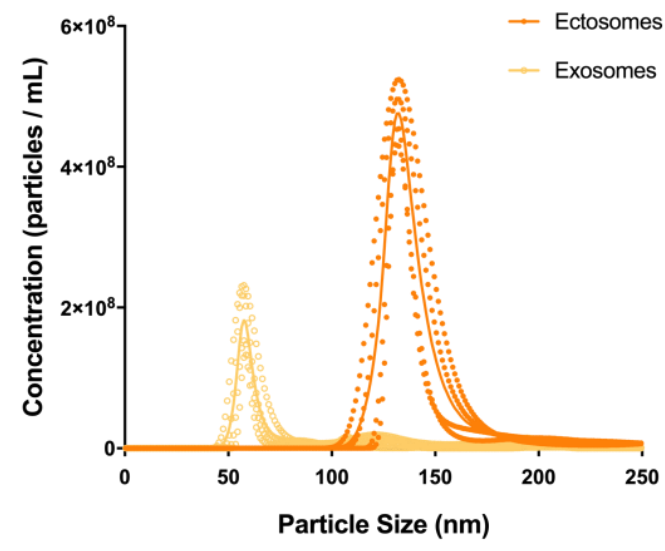

D

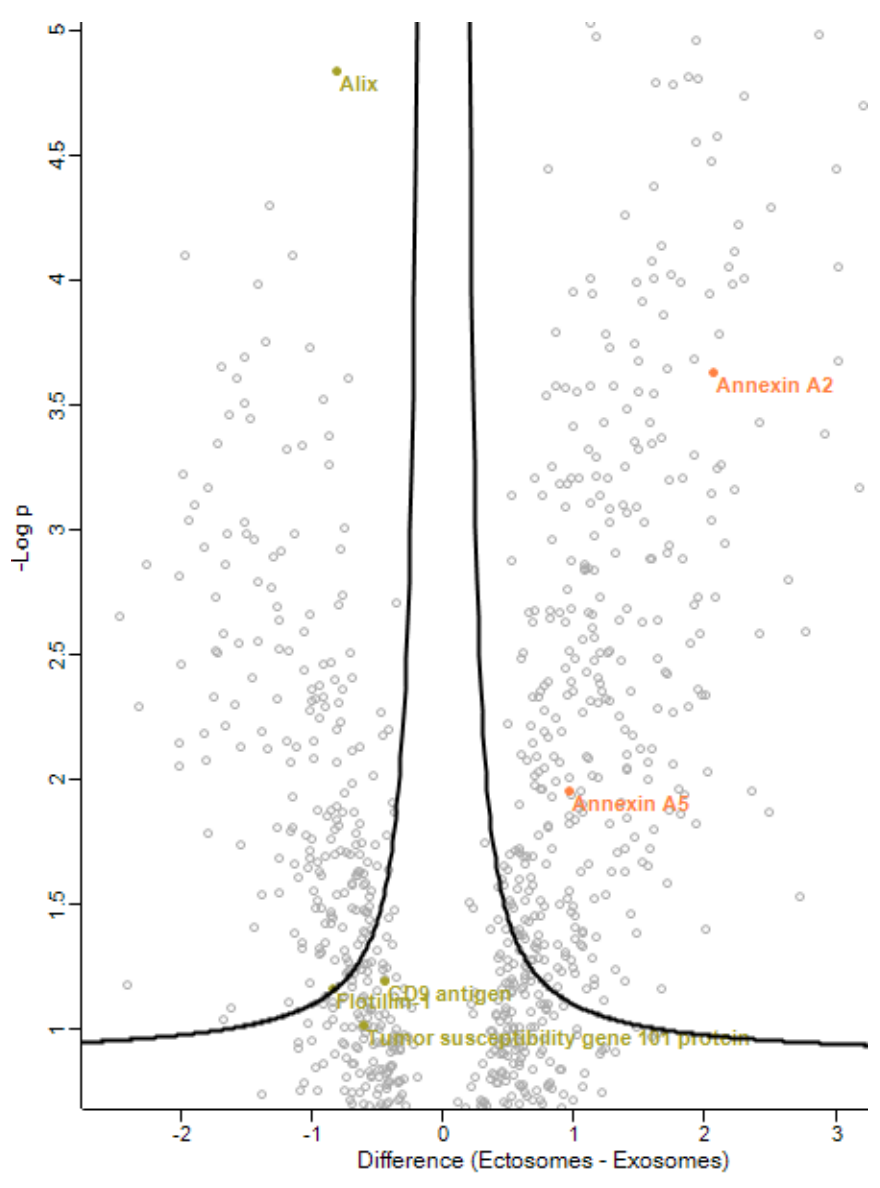

\section{Supplementary Figure 2. Purification and characterization of secreted EVs using differential centrifugation.}

(A) MemCode staining demonstrates the total protein levels present in each fraction. Exosome-depleted cell media was collected from HEK cells after 24 hours and subsequently centrifuged at different speed. (B) Whole-mount electron microscopy analysis of each pellet showing representative images of ectosomes and exosomes (scale bar $100 \mathrm{~nm}$ ). (C) Nanoparticle tracking analysis (NTA) measurements of particle concentrations and average size distributions of ectosomes and exosomes. Average is represented with the filled line while each dotted line represents one biological replicate. Yellow dots represent exosomes measurements, while orange dots represent ectosomes measurements. Data from at least three independent experiments for each condition. (D) Proteomic analyses of ectosomes and exosomes using label-free quantitative mass spectrometry demonstrates the enrichment of specific protein markers in each fraction. Yellow dots represent the proteins enrichment in exosomes, while orange dots represent enrichment in ectosomes. Dots above the volcano plot line represent proteins for which differences were significant (false discovery rate $[F D R]<0.1$ ). Data represented in "t-test Difference (Ectosomes Exosomes)" vs. "-Log t-test p-value" from 5 independent samples for each group. Data analyses were performed using Perseus software. 

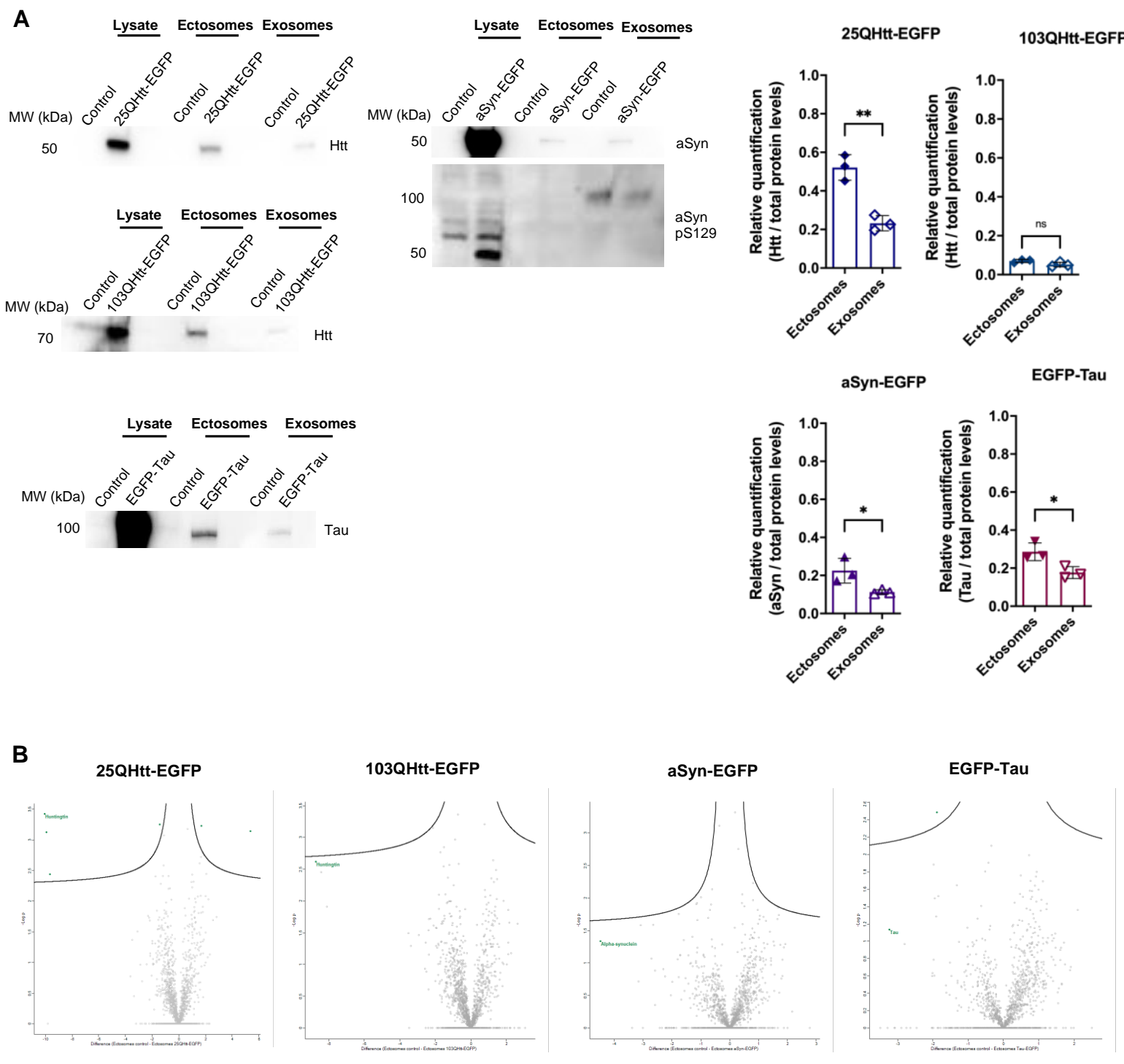

C
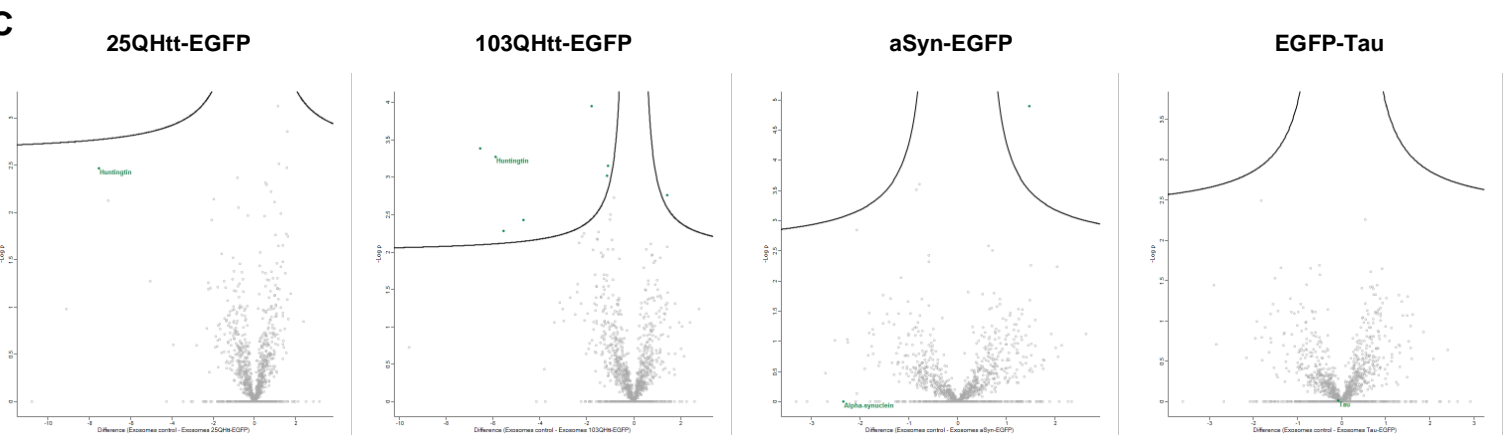

Supplementary Figure 3. Disease-related proteins are enriched in ectosomes. (A) Immunoblots of ectosomes and exosomes purified from the media of HEK cells stably expressing 25QHtt-EGFP, 103QHtt-EGFP, aSyn-EGFP or EGFP-Tau for 24 hours. Equal quantities of protein were separated on SDS-PAGE gels, and membranes were blotted with the indicated antibodies. Protein levels were normalized to total protein levels using Memcode. Data from at least three independent experiments for each condition. Significant differences were assessed by two-tailed unpaired $t$ test comparison and are expressed as mean $\pm S D,{ }^{*} p<0.05$, ${ }^{* *} p<0.01$. (B-C) Proteomic analyses of ectosomes and exosomes using label-free quantitative mass spectrometry demonstrates the enrichment of 25QHttEGFP, 103QHtt-EGFP, aSyn-EGFP and EGFP-Tau in ectosomes (B) and exosomes (C) compared with the control 
(proteins are identified in green). (B) Data represented in "t-test Difference (Ectosomes - Ectosomes 25QHtt-EGFP/ 103QHtt-EGFP/ aSyn-EGFP/ EGFP-Tau)” vs. "-Log t-test p-value” from 3 independent samples for each group. (C) Data represented in "t-test Difference (Exosomes - Exosomes 25QHtt-EGFP/ 103QHtt-EGFP/ aSyn-EGFP/ EGFPTau)" vs. "-Log t-test p-value" from 3 independent samples for each group. Dots above the volcano plot line represent proteins for which differences were significant (false discovery rate $[F D R]<0.1$ ). Data analyses were performed using Perseus software.

A

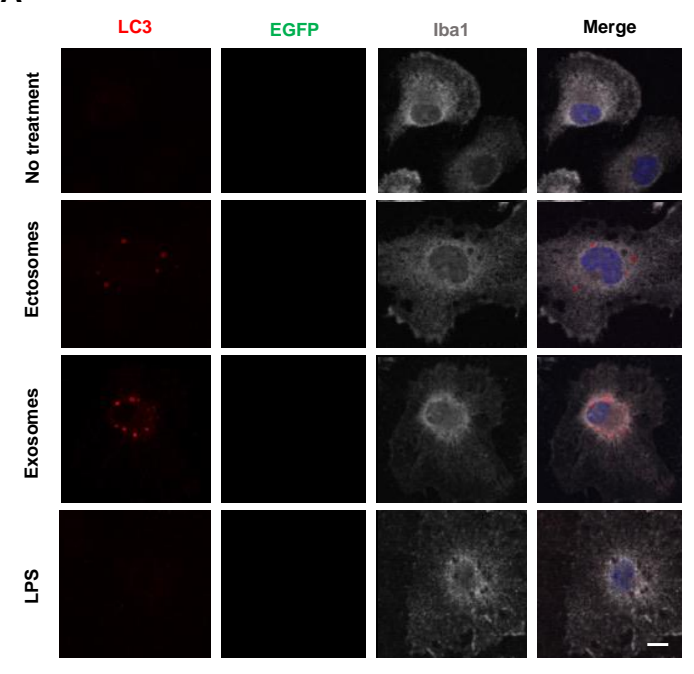

C
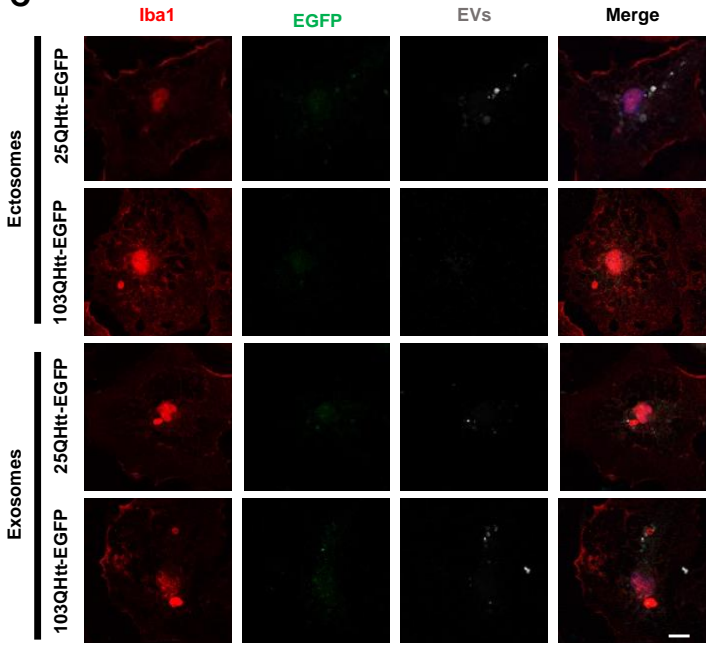

B
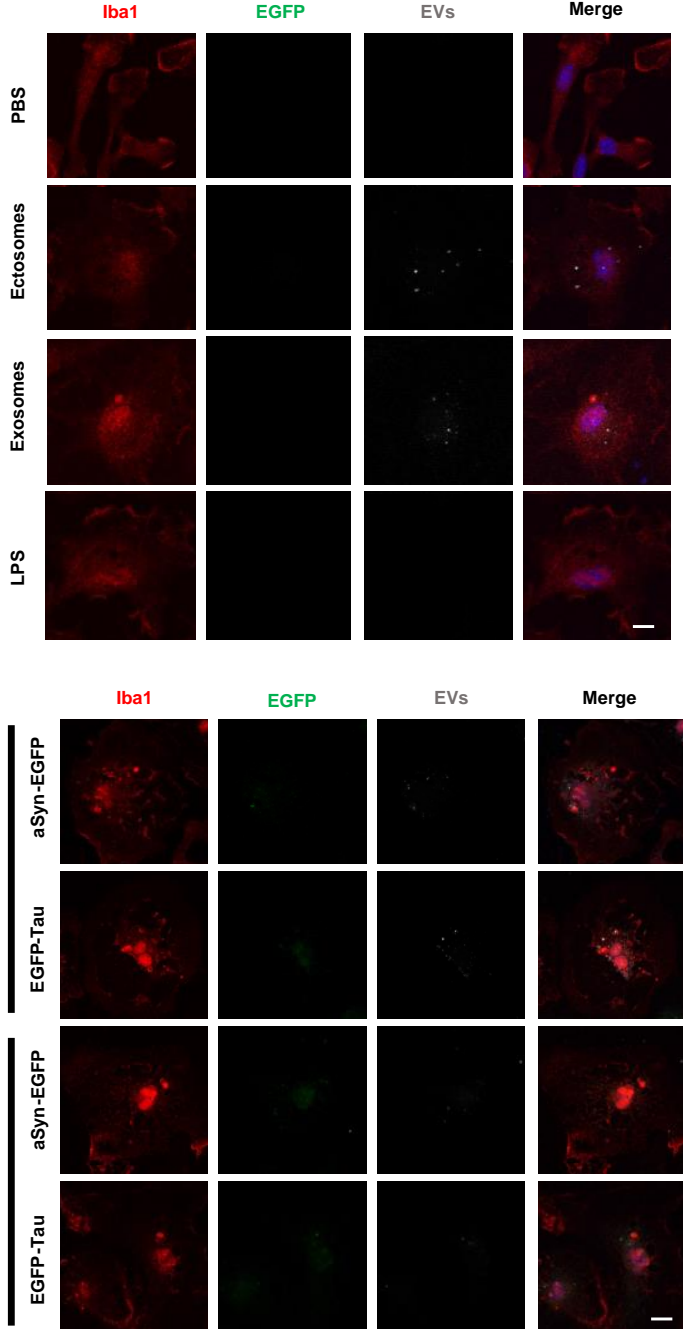

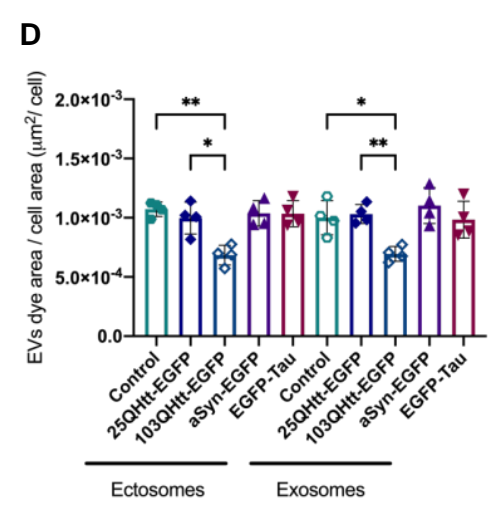

Supplementary Figure 4. Ectosomes and exosomes containing disease-related proteins are internalized by microglial cells. (A) Ectosomes and exosomes were applied to microglial cultures at a concentration of $10 \mu \mathrm{g} / \mathrm{mL}$ 
for 24 hours. Cells were immunostained for LC3 (red) and lba1 (grey). Scale bar $10 \mu \mathrm{m}$. (B) Ectosomes and exosomes were labelled with Alexa Fluor 633 C5-maleimide (grey) and applied to microglial cultures at a concentration of $10 \mu \mathrm{g} / \mathrm{mL}$ for 24 hours. Cells were immunostained for lba1 (red). Scale bar $10 \mu \mathrm{m}$. (C) Ectosomes and exosomes containing 25QHtt-EGFP, 103QHtt-EGFP, aSyn-EGFP or EGFP-Tau were labelled with Alexa Fluor 633 C5-maleimide (grey) and applied to microglial cultures at a concentration of $10 \mu \mathrm{g} / \mathrm{mL}$ for 24 hours. Cells were immunostained for lba1 (red). Scale bar $10 \mu \mathrm{m}$. (D) EV internalization was evaluated through imaging analysis by measuring fluorescence intensity and cell area. Data from at least three independent experiments for each condition. Significant differences were assessed by one-way ANOVA followed by multiple comparisons with significance between groups corrected by Bonferroni procedure. Differences were considered to be significant for values of $p<0.05$ and are expressed as mean $\pm S D,{ }^{*} p<0.05,{ }^{* *} p<0.01$.
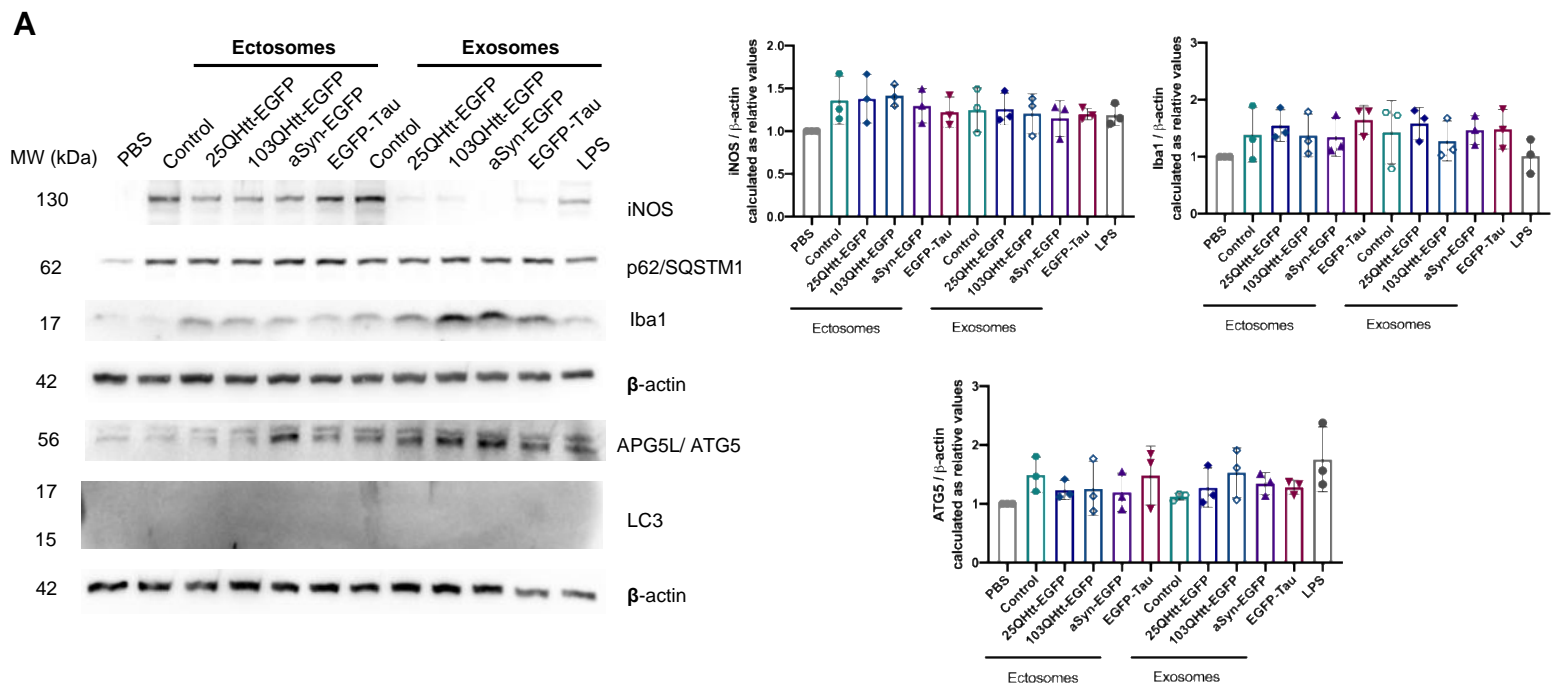

B

IL-10

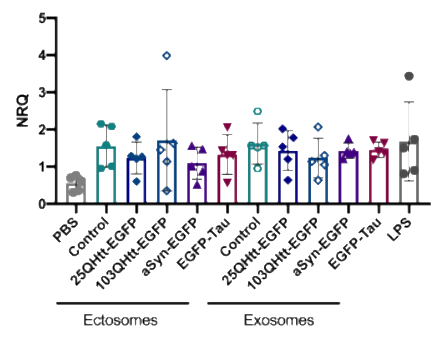

TNFa

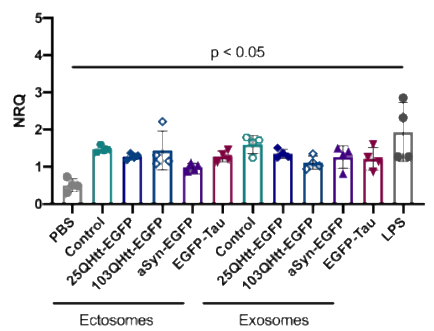

IL-6

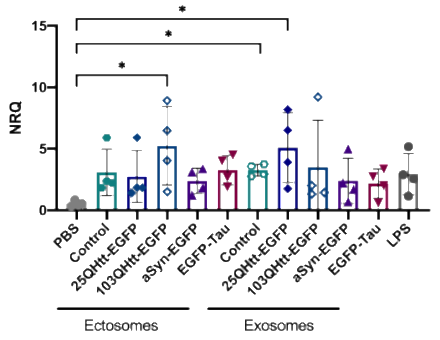

C
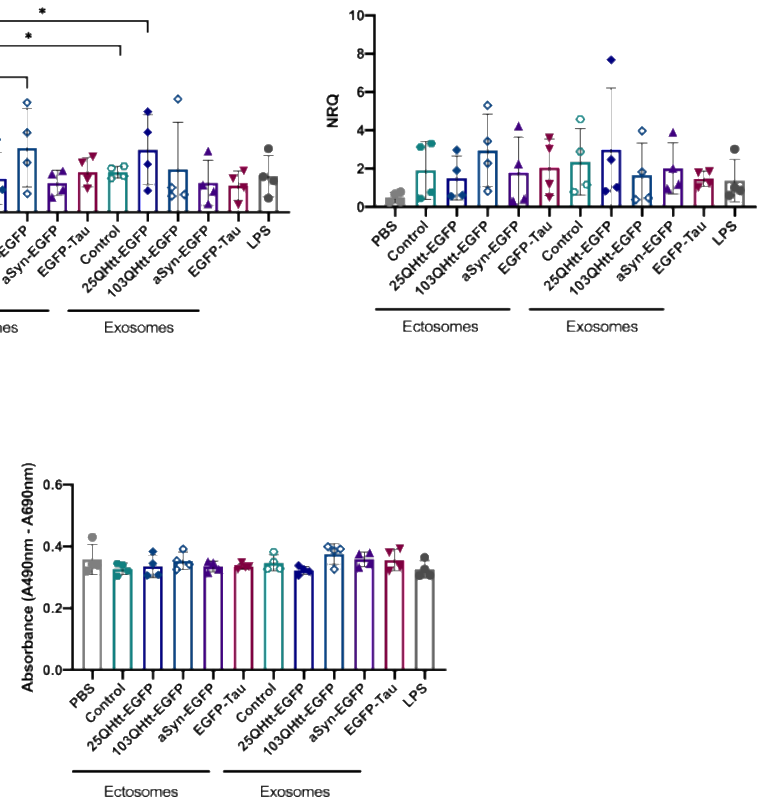

Supplementary Figure 5. Ectosomes and exosomes containing disease-related proteins are internalized by microglial cells. (A) Ectosomes and exosomes containing 25QHtt-EGFP, 103QHtt-EGFP, aSyn-EGFP or EGFPTau were applied to microglial cultures at a concentration of $10 \mu \mathrm{g} / \mathrm{mL}$ for 24 hours. Immunoblot and protein 
quantifications of iNOS, Iba1 and APG5L/ ATG5. (B) EV treatment leads to the activation of the pro-inflammatory markers IL-6 and TNFa in microglia cells after 24hours. (C) LDH measurements confirm the absence of cell toxicity and cell death in the experiments. Data from at least three independent experiments for each condition. Significant differences were assessed by one-way ANOVA followed by multiple comparisons with significance between groups corrected by Bonferroni procedure. Differences were considered to be significant for values of $p<0.05$ and are expressed as mean $\pm S D,{ }^{*} p<0.05$.

A

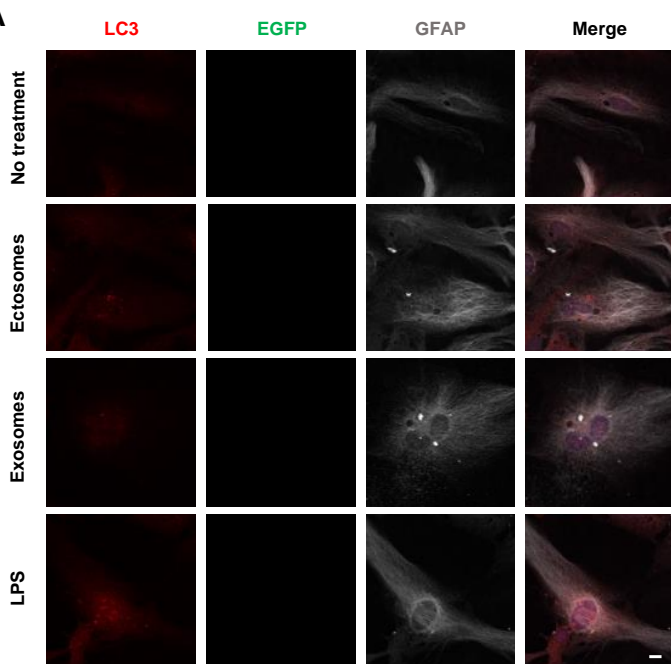

C
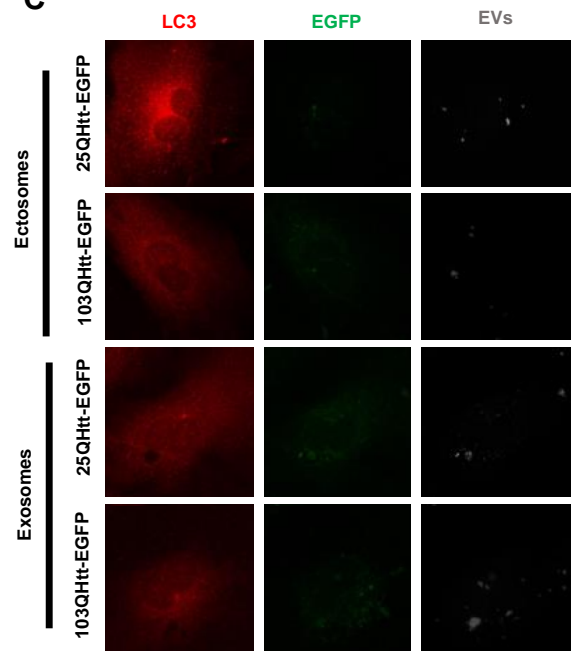

Merge

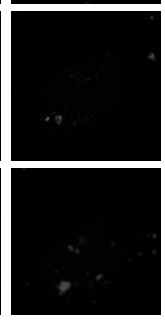

B

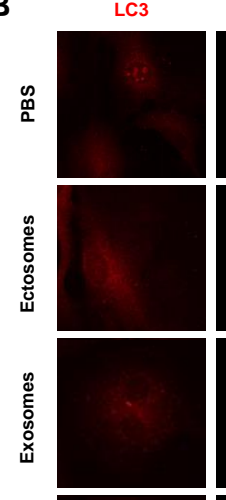

$\stackrel{\infty}{3}$

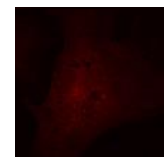

LC3
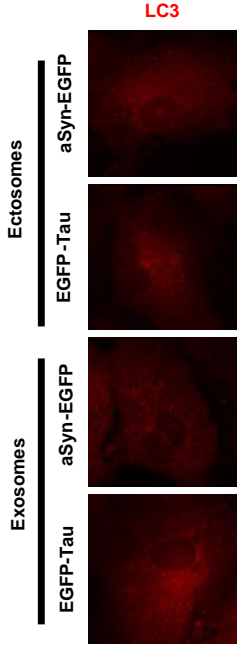

EGFP
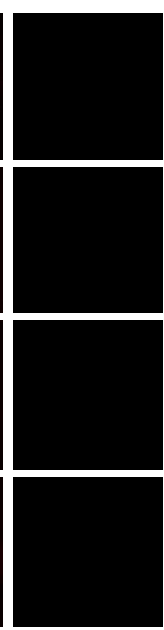

EGFP
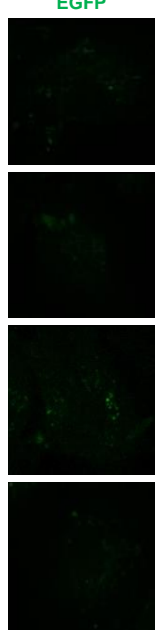

EVs
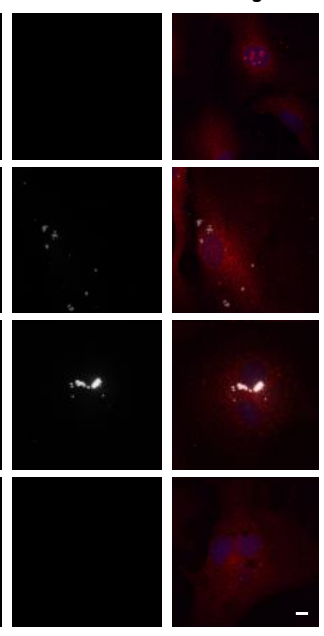

me
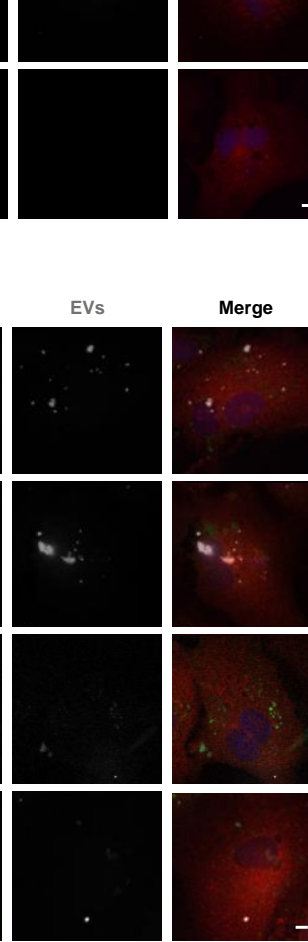

Merge
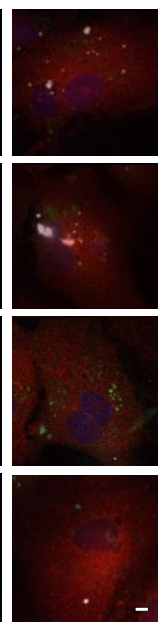

D

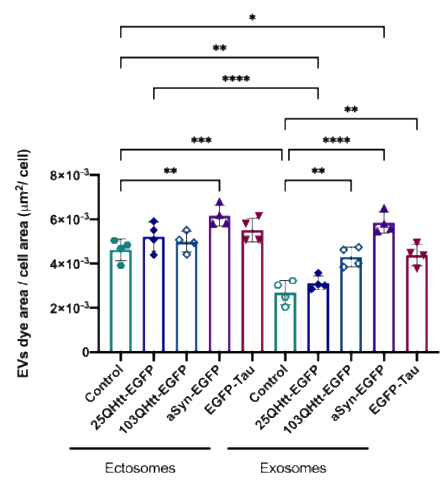

Supplementary Figure 6. Ectosomes and exosomes containing disease-related proteins are internalized by astrocytic cells. (A) Ectosomes and exosomes were applied to astrocytic cultures at a concentration of $20 \mu \mathrm{g} / \mathrm{mL}$ 
for 24 hours. Cells were immunostained for LC3 (red) and GFAP (grey). Scale bar $10 \mu \mathrm{m}$. (B) Ectosomes and exosomes were labelled with Alexa Fluor 633 C5-maleimide (grey) and applied to astrocytic cultures at a concentration of $20 \mu \mathrm{g} / \mathrm{mL}$ for 24 hours. Cells were immunostained for lba1 (red). Scale bar $10 \mu \mathrm{m}$. (C) Ectosomes and exosomes containing 25QHtt-EGFP, 103QHtt-EGFP, aSyn-EGFP or EGFP-Tau were labelled with Alexa Fluor 633 C5-maleimide (grey) and applied to cell cultures at a concentration of $20 \mu \mathrm{g} / \mathrm{mL}$ for 24 hours. Cells were immunostained for lba1 (red). Scale bar $10 \mu \mathrm{m}$. (D) EV internalization levels were evaluated by image analyses measuring fluorescence intensity and cell area. Data from at least three independent experiments for each condition. Significant differences were assessed by one-way ANOVA followed by multiple comparisons with significance between groups corrected by Bonferroni procedure. Differences were considered to be significant for values of $p<0.05$ and are expressed as mean $\pm S D,{ }^{*} p<0.05,{ }^{* *} p<0.01,{ }^{* *} p<0.001,{ }^{* \star * *} p<0.0001$. 

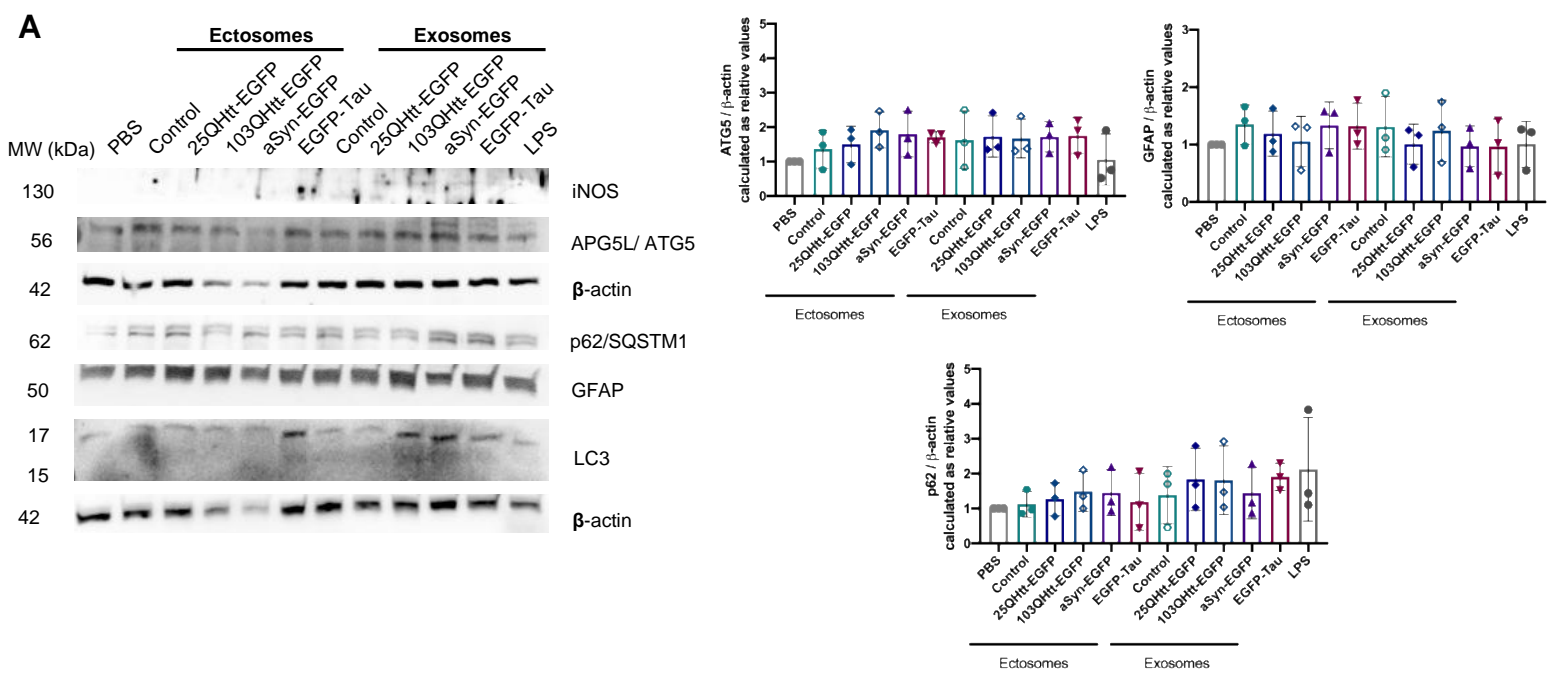

B

IL- $\beta$

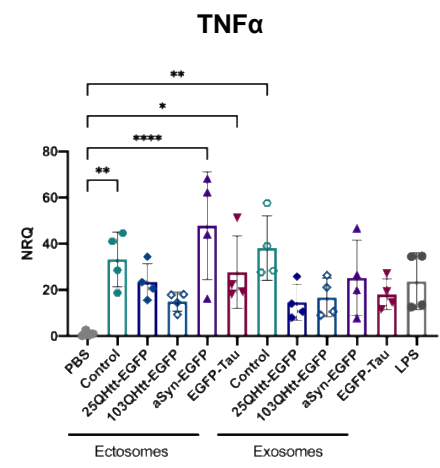

C
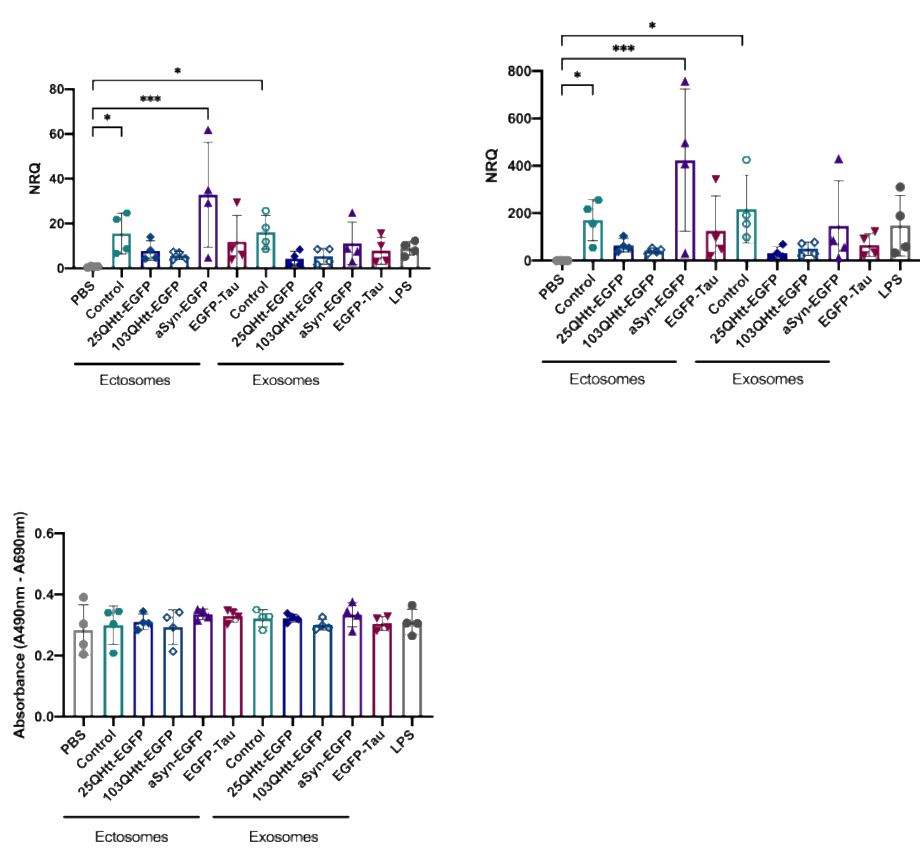

Supplementary Figure 7. Ectosomes and exosomes containing disease-related proteins are internalized by astrocytic cells. (A) Ectosomes and exosomes containing 25QHtt-EGFP, 103QHtt-EGFP, aSyn-EGFP or EGFPTau were applied into astrocytic cultures at a concentration of $20 \mu \mathrm{g} / \mathrm{mL}$ for 24 hours. Immunoblot and protein quantifications of iNOS, APG5L/ ATG5, p62, GFAP and LC3. (B) EV treatment leads to the activation of the proinflammatory markers IL-6, IL- $\beta$ and TNFa in astrocytic cells after 24hours. (C) LDH measurements confirm the absence of cell toxicity and cell death in the experiments. Data from at least three independent experiments for each condition. Significant differences were assessed by one-way ANOVA followed by multiple comparisons with significance between groups corrected by Bonferroni procedure. Differences were considered to be significant for values of $p<0.05$ and are expressed as mean $\pm S D,{ }^{*} p<0.05,{ }^{* *} p<0.01,{ }^{* * *} p<0.001,{ }^{* * * *} p<0.0001$. 

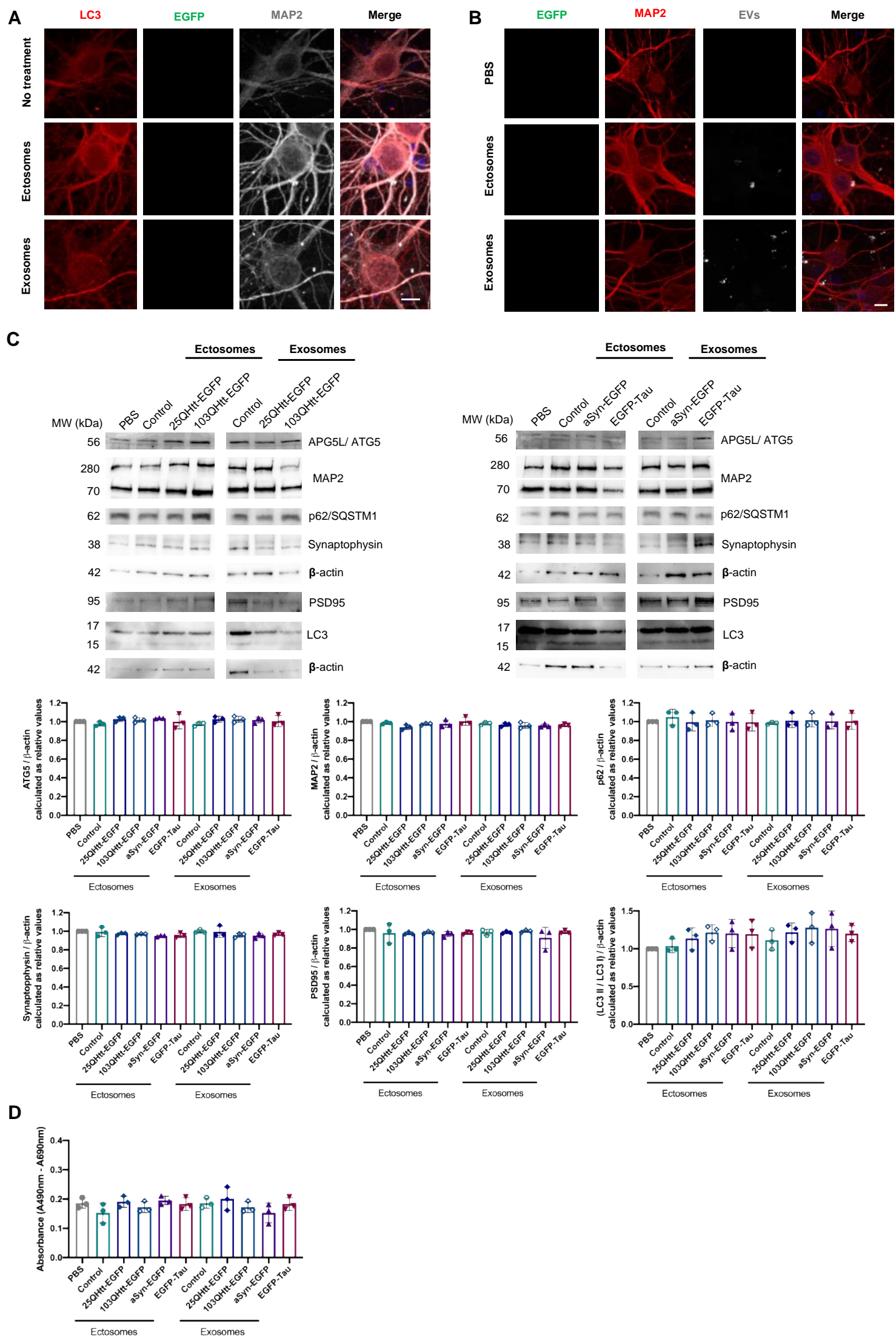

Supplementary Figure 8. Ectosomes and exosomes containing disease-related proteins are internalized by primary cortical neurons. (A) Ectosomes and exosomes were applied to primary cortical neurons $20 \mu \mathrm{g} / \mathrm{mL}$ for 24 hours. Cells were immunostained for LC3 (red) and GFAP (grey). Scale bar $5 \mu \mathrm{m}$. (B) Ectosomes and exosomes 
were labelled with Alexa Fluor 633 C5-maleimide (grey) and applied to neuronal cultures at a concentration of $20 \mu \mathrm{g} / \mathrm{mL}$ for 24 hours. Cells were immunostained for MAP2 (red). Scale bar $5 \mu \mathrm{m}$. (C) Ectosomes and exosomes containing 25QHtt-EGFP, 103QHtt-EGFP, aSyn-EGFP or EGFP-Tau were applied to primary cortical cultures at a concentration of $20 \mu \mathrm{g} / \mathrm{mL}$ for 24 hours. Immunoblot and protein quantifications of PSD95, synaptophysin, MAP2, APG5L/ ATG5, p62, and LC3. (D) LDH measurements confirm the absence of cell toxicity and cell death in the experiments. Data from at least three independent experiments for each condition. Significant differences were assessed by one-way ANOVA followed by multiple comparisons with significance between groups corrected by Bonferroni procedure. Differences were considered to be significant for values of $p<0.05$ and are expressed as mean \pm SD. 


\section{Discussion}

Neurodegenerative disorders are characterized by progressive loss of specific vulnerable neuronal populations, and are classified according to clinical features, anatomic distribution of pathology, and molecular mechanisms involved in the disease progression. The most common neurodegenerative disorders are amyloidosis, tauopathies, alphasynucleinopathies, and polyQ proteinopathies [4]. The accumulation of abnormal protein conformations in the brain is a common hallmark in these disorders. Recently, the specific anatomical pattern of disease progression in neurodegenerative diseases has been partially explained through the transmission of abnormal protein conformers along anatomically connected pathways in the brain. This includes aSyn in PD and other synucleinopathies, Tau in tauopathies and $\mathrm{mHtt}$ in HD. Diverse molecular mechanisms seem to be involved in this process, contributing for disease progression and neurodegeneration. However, their functional consequences and contribution for the development of the pathology are only partially understood. Therefore, it is important to establish differences and similarities in the pathways that aSyn, Tau and Htt are handled in order to identify specific therapeutic targets for each disease.

Protein secretion is often associated with intracellular accumulation of misfolded proteins. The cellular burden of aggregated proteins can induce the activation of several mechanisms that aim to reduce their levels in the cells and promote protein secretion to the extracellular milieu. However, as we present in our work, proteins can also be physiologically released from cells unrelated to protein aggregation. This hypothesis might explain the inconsistencies regarding protein spreading described in the literature. However, further research is necessary to evaluate the physiological role of aSyn, Tau and Htt secretion.

In our work, we demonstrate the release of distinct disease-related proteins through similar prion-like spreading mechanisms. Despite the diverse functional roles of aSyn, Tau and $\mathrm{Htt}$, cells direct these proteins to ER, MVBs and plasma membrane for their secretion. Overall, these results indicate that prion-like spreading mechanisms are common to several neurodegenerative diseases and may not be restricted to a specific protein or disease. Although, proteins can be sorted more favourably for one of these mechanisms since we observed differences between the secretion and internalization levels of aSyn, Tau and Htt. Additionally, cell-to-cell transmission can depend on the protein intrinsic properties, cellular function, and their potential role in the extracellular milieu.

Protein secretion involves non-vesicular and vesicular-mediated pathways. In our study, aSyn, Tau and $\mathrm{Htt}$ were present in the cell media both as free proteins and associated with EVs. In particular, release of aSyn, Tau and normal $\mathrm{Htt}$ in a free form occurred in higher 
levels compared with $\mathrm{mHtt}$. The size, distribution, and stability of $\mathrm{mHtt}$ might affect its secretion through the plasma membrane or using the MAPS pathway. Interestingly, the percentage of cells that internalized Tau were higher when compared with the other proteins, despite Tau not being the protein more exported to the extracellular space. This indicates that Tau might be more easily/ faster internalized in cells, or additional pathways might be involved in its uptake compared with the other proteins. It is also possible the existence of protein degradation in the extracellular space by proteases. Additionally, it demonstrates that protein internalization is not a process entirely dependent of the protein levels in the exterior space, but it might be determined by the type of protein, receptor cell, and mechanisms involved in the cellular uptake.

The role of EVs in health and age-associated diseases is far from being completely understood. These membrane-enclosed particles are released by several cell types in the brain and are central for intercellular communication. EVs are a selective pathway for the physiological transfer of nucleic acids, lipids and proteins, but also for the elimination of misfolded proteins. Recently, evidence implicated these vesicles as causative players in the spreading of neurodegenerative diseases but also as relevant biomarkers for these disorders. Cells release a variety of EVs that differ in size, morphology, composition, and mechanisms of biogenesis. However, the use of different EVs purification methods, absence of reliable markers and lack of comprehensive characterization resulted in numerous contradictory data and challenges in their study.

Numerous studies have been focusing on the characterization and biological function of exosomes. However, ectosomes have been described in the literature as important EVs in $A D$ and remain largely understudied. In our work, we provide a reproducible differential ultracentrifugation protocol optimized to efficiently isolate ectosomes and exosomes from diverse biofluids. Comprehensive proteomic and molecular analysis of the different EVs subtypes revealed singular proteomic profiles and displayed their individual biological and molecular signatures. Notably, we have identified several membrane-associated proteins annexins as novel markers for ectosomes, in particular annexin-A2. We also demonstrate that ectosomes and exosomes are internalized in similar quantities in cortical neurons and can be transported through the axons to other cells. Still, other EVs content may be altered as lipids and nucleic acids. We further demonstrate that spontaneous neuronal function can be differently modulated by ectosomes and exosomes, and that EVs uptake is associated with a disruption of the typical synchronized bursting neuronal activity. Overall, these results indicate that ectosomes and exosomes not only have different biogenesis and protein content, but also specific functional effects in physiological conditions. 
Interestingly, ectosomes exhibited higher levels of aSyn, Tau and Htt compared with exosomes. Also, incorporation of these proteins in EVs did not change the vesicles protein composition. The internalization of disease-related proteins in EVs can be a protective mechanism for clearance of aggregation-prone proteins and possible be involved in the spreading of pathology in the brain. This is context, these vesicles may also play a role in signalling intracellular changes taking place during the disease process to the neighbouring cells.

Once in the extracellular space, aSyn, Tau and $\mathrm{Htt}$ in a free form or in EVs have distinct functional consequences in cortical neurons. Treatment with monomeric aSyn, Tau and $\mathrm{Htt}$ resulted in the modulation of the spontaneous neuronal activity depending on the protein added to the culture, in contrast to EVs where the alterations were mainly correlated with the EVs type. This might indicate different functional effects of aSyn, Tau and $\mathrm{Htt}$ in neuronal cells when present in a free form and in EVs. However, aSyn, Tau and Htt were present in lower levels in EVs compared with the total levels in the cell media, potentially explaining the distinct functional effects observed. Indeed, we observed sight differences in the spontaneous neuronal activity in cells treated with ectosomes and exosomes containing $25 \mathrm{QH} t \mathrm{tt}$ and 103QHtt. Also, different mechanisms may be involved in the internalization of these proteins in a free form or in EVs, possibly leading to distinctive functional effects.

Protein secretion in EVs might contribute to a variety of detrimental effects in receiving cells, not only in the spontaneous activity in neuronal cells, but also in the activation of autophagic and inflammatory processes in microglial and astrocytic cells. It is also conceivable the existence of specificity between the EVs type and the receptor cells, since we observed different internalization levels for EVs in neurons, microglia and astrocytes. Altogether, our results emphasize the potential distinct effects of ectosomes and exosomes in physiological and pathological conditions.

Many studies suggest that aSyn, Tau and Htt have prion-like properties, however there are several topics that need further clarification. One of the main arguments against this hypothesis is the lack of studies demonstrating protein infectivity between individuals, and evidence regarding endogenous protein transmission cell-to-cell. Also, PrP, aSyn, Tau and $\mathrm{Htt}$ have different protein functions and distribution in cell types and in the brain. Although the passive release of misfolded proteins from damaged or dead neurons is considered insignificant, these levels may be enough for the development of pathology prior to the manifestation of the typical motor features. Lastly, the proposed prion-like spreading mechanisms already exist in physiological conditions, implying that they do not have a role only in pathology progression. 
Our current understanding suggests that prion-like mechanisms may not be the main driving factor in neurodegenerative diseases, but that they might contribute to the acceleration of disease progression. Also, it is unknow the factors promoting progressive cell-to-cell transfer of proteins, and if this is a physiological or pathological process. Our work highlights the involvement of common molecular mechanisms in the transmission of aSyn, Tau and $\mathrm{Htt}$ in neurodegenerative diseases, and emphasizes potential biomarkers and targets for the development of novel therapeutic strategies. 


\section{Conclusions}

Herein, we provide evidence that disease-related proteins found to be important in several neurodegenerative diseases may transfer from cell-to-cell via similar mechanisms, and therefore negatively impacting on the neighbouring cells and contributing for disease progression. Although each neurogenerative disease is usually presented as a distinct entity, they often overlap in several features.

The functional and toxic consequences of aSyn, Tau and $\mathrm{Htt}$ in the extracellular space are dependent not only on each of their properties, but also in the mechanisms involved in their release. Particularly, we emphasize that protein release in a free form or in EVs might contribute to a variety of detrimental effects in receptor cells and to pathology progression. However, protein secretion may also constitute an important physiological process in the brain.

Understanding the function of EVs under physiological and pathological conditions will unravel the molecular mechanisms underlying dementias and assist in the development of disease-modifying strategies. Also, it is important when considering the development of therapeutic strategies to avoid interfering with the normal physiological intercellular communication. Studies focusing in EVs are essential for opening new avenues for their clinical use as biomarkers, and as vehicles for the targeted delivery of compounds and other therapies.

An immense advance has been achieved in the field over the last years regarding the understanding of the molecular and cellular mechanisms that regulate protein release and internalization, and whether and how these contribute to the pathology transmission in neurodegenerative diseases. Despite several important questions remain unanswered, these are exciting times in research, as today we now more than when the prion-like hypothesis was proposed. 


\section{References}

1. Heemels, M.T., Neurodegenerative diseases. Nature, 2016. 539(7628): p. 179.

2. Hinz, F.I. and D.H. Geschwind, Molecular Genetics of Neurodegenerative Dementias. Cold Spring Harb Perspect Biol, 2017. 9(4).

3. Eisenberg, D. and M. Jucker, The amyloid state of proteins in human diseases. Cell, 2012. 148(6): p. 1188-203.

4. Dugger, B.N. and D.W. Dickson, Pathology of Neurodegenerative Diseases. Cold Spring Harb Perspect Biol, 2017. 9(7).

5. Dugger, B.N., et al., Clinicopathological outcomes of prospectively followed normal elderly brain bank volunteers. J Neuropathol Exp Neurol, 2014. 73(3): p. 244-52.

6. Dugger, B.N., et al., Concomitant pathologies among a spectrum of parkinsonian disorders. Parkinsonism Relat Disord, 2014. 20(5): p. 525-9.

7. Chiti, F. and C.M. Dobson, Protein Misfolding, Amyloid Formation, and Human Disease: A Summary of Progress Over the Last Decade. Annu Rev Biochem, 2017. 86: p. 27-68.

8. Braak, H. and E. Braak, Neuropathological stageing of Alzheimer-related changes. Acta Neuropathol, 1991. 82(4): p. 239-59.

9. Braak, H. and E. Braak, Staging of Alzheimer's disease-related neurofibrillary changes. Neurobiol Aging, 1995. 16(3): p. 271-8; discussion 278-84.

10. Braak, H., et al., Staging of brain pathology related to sporadic Parkinson's disease. Neurobiol Aging, 2003. 24(2): p. 197-211.

11. Braak, H., et al., Stages in the development of Parkinson's disease-related pathology. Cell Tissue Res, 2004. 318(1): p. 121-34.

12. Vonsattel, J.P., et al., Neuropathological classification of Huntington's disease. J Neuropathol Exp Neurol, 1985. 44(6): p. 559-77.

13. Spillantini, M.G. and M. Goedert, Synucleinopathies: past, present and future. Neuropathol Appl Neurobiol, 2016. 42(1): p. 3-5.

14. Bras, I.C., M. Xylaki, and T.F. Outeiro, Mechanisms of alpha-synuclein toxicity: An update and outlook. Prog Brain Res, 2020. 252: p. 91-129.

15. Spillantini, M.G., et al., Alpha-synuclein in Lewy bodies. Nature, 1997. 388(6645): p. 839-40.

16. Wakabayashi, $\mathrm{K}$., et al., NACP, a presynaptic protein, immunoreactivity in Lewy bodies in Parkinson's disease. Neurosci Lett, 1997. 239(1): p. 45-8.

17. Arawaka, S., et al., Lewy body in neurodegeneration with brain iron accumulation type 1 is immunoreactive for alpha-synuclein. Neurology, 1998. 51(3): p. 887-9.

18. Gai, W.P., et al., Multiple-system atrophy: a new alpha-synuclein disease? Lancet, 1998. 352(9127): p. 547-8.

19. Parkinson, J., An essay on the shaking palsy. 1817. J Neuropsychiatry Clin Neurosci, 2002. 14(2): p. 223-36; discussion 222.

20. Jenner, P., et al., Parkinson's disease--the debate on the clinical phenomenology, aetiology, pathology and pathogenesis. J Parkinsons Dis, 2013. 3(1): p. 1-11.

21. Postuma, R.B., et al., MDS clinical diagnostic criteria for Parkinson's disease. Mov Disord, 2015. 30(12): p. 1591-601.

22. Dauer, W. and S. Przedborski, Parkinson's disease: mechanisms and models. Neuron, 2003. 39(6): p. 889-909. 
23. Gibb, W.R. and A.J. Lees, The relevance of the Lewy body to the pathogenesis of idiopathic Parkinson's disease. J Neurol Neurosurg Psychiatry, 1988. 51(6): p. 745-52.

24. Poewe, W., et al., Parkinson disease. Nat Rev Dis Primers, 2017. 3: p. 17013.

25. Lewy, F., Paralysis agitans., in Handbuch der Neurologie, A.G. Lewandowsky M, Editor. 1912, Springer Verlag: Berlin. p. 920-933.

26. Spillantini, M.G., et al., alpha-Synuclein in filamentous inclusions of Lewy bodies from Parkinson's disease and dementia with lewy bodies. Proc Natl Acad Sci U S A, 1998. 95(11): p. 6469-73.

27. Lavedan, C., The synuclein family. Genome Res, 1998. 8(9): p. 871-80.

28. Clayton, D.F. and J.M. George, The synucleins: a family of proteins involved in synaptic function, plasticity, neurodegeneration and disease. Trends Neurosci, 1998. 21(6): p. 249-54.

29. Jakes, R., M.G. Spillantini, and M. Goedert, Identification of two distinct synucleins from human brain. FEBS Lett, 1994. 345(1): p. 27-32.

30. Nakajo, S., et al., Purification and characterization of a novel brain-specific 14-kDa protein. J Neurochem, 1990. 55(6): p. 2031-8.

31. Abeliovich, A., et al., Mice lacking alpha-synuclein display functional deficits in the nigrostriatal dopamine system. Neuron, 2000. 25(1): p. 239-52.

32. Chandra, S., et al., Double-knockout mice for alpha- and beta-synucleins: effect on synaptic functions. Proc Natl Acad Sci U S A, 2004. 101(41): p. 14966-71.

33. Anwar, S., et al., Functional alterations to the nigrostriatal system in mice lacking all three members of the synuclein family. J Neurosci, 2011. 31(20): p. 7264-74.

34. Greten-Harrison, B., et al., alphabetagamma-Synuclein triple knockout mice reveal age-dependent neuronal dysfunction. Proc Natl Acad Sci U S A, 2010. 107(45): p. 19573-8.

35. Burre, J., et al., Alpha-synuclein promotes SNARE-complex assembly in vivo and in vitro. Science, 2010. 329(5999): p. 1663-7.

36. Maroteaux, L., J.T. Campanelli, and R.H. Scheller, Synuclein: a neuron-specific protein localized to the nucleus and presynaptic nerve terminal. J Neurosci, 1988. 8(8): p. 280415.

37. Withers, G.S., et al., Delayed localization of synelfin (synuclein, NACP) to presynaptic terminals in cultured rat hippocampal neurons. Brain Res Dev Brain Res, 1997. 99(1): p. 87-94.

38. Hsu, L.J., et al., Expression pattern of synucleins (non-Abeta component of Alzheimer's disease amyloid precursor protein/alpha-synuclein) during murine brain development. J Neurochem, 1998. 71(1): p. 338-44.

39. Bayer, T.A., et al., Neural expression profile of alpha-synuclein in developing human cortex. Neuroreport, 1999. 10(13): p. 2799-803.

40. Galvin, J.E., et al., Differential expression and distribution of alpha-, beta-, and gammasynuclein in the developing human substantia nigra. Exp Neurol, 2001. 168(2): p. 34755.

41. Chu, Y. and J.H. Kordower, Age-associated increases of alpha-synuclein in monkeys and humans are associated with nigrostriatal dopamine depletion: Is this the target for Parkinson's disease? Neurobiol Dis, 2007. 25(1): p. 134-49.

42. Collier, T.J., N.M. Kanaan, and J.H. Kordower, Aging and Parkinson's disease: Different sides of the same coin? Mov Disord, 2017. 32(7): p. 983-990. 
43. Hasegawa, M., T. Nonaka, and M. Masuda-Suzukake, alpha-Synuclein: Experimental Pathology. Cold Spring Harb Perspect Med, 2016. 6(9).

44. Burre, J., The Synaptic Function of alpha-Synuclein. J Parkinsons Dis, 2015. 5(4): p. 699713.

45. Chen, $\mathrm{X}$., et al., The human NACP/alpha-synuclein gene: chromosome assignment to 4q21.3-q22 and TaqI RFLP analysis. Genomics, 1995. 26(2): p. 425-7.

46. Polymeropoulos, M.H., et al., Mutation in the alpha-synuclein gene identified in families with Parkinson's disease. Science, 1997. 276(5321): p. 2045-7.

47. Kruger, R., et al., Ala30Pro mutation in the gene encoding alpha-synuclein in Parkinson's disease. Nat Genet, 1998. 18(2): p. 106-8.

48. Zarranz, J.J., et al., The new mutation, E46K, of alpha-synuclein causes Parkinson and Lewy body dementia. Ann Neurol, 2004. 55(2): p. 164-73.

49. Kiely, A.P., et al., alpha-Synucleinopathy associated with G51D SNCA mutation: a link between Parkinson's disease and multiple system atrophy? Acta Neuropathol, 2013. 125(5): p. 753-69.

50. Proukakis, C., et al., A novel alpha-synuclein missense mutation in Parkinson disease. Neurology, 2013. 80(11): p. 1062-4.

51. Pasanen, P., et al., Novel alpha-synuclein mutation A53E associated with atypical multiple system atrophy and Parkinson's disease-type pathology. Neurobiol Aging, 2014. 35(9): p. 2180 e1-5.

52. Liu, H., et al., A Novel SNCA A30G Mutation Causes Familial Parkinson's Disease. Mov Disord, 2021.

53. Singleton, A.B., et al., alpha-Synuclein locus triplication causes Parkinson's disease. Science, 2003. 302(5646): p. 841.

54. Ibanez, P., et al., Causal relation between alpha-synuclein gene duplication and familial Parkinson's disease. Lancet, 2004. 364(9440): p. 1169-71.

55. Ferese, R., et al., Four Copies of SNCA Responsible for Autosomal Dominant Parkinson's Disease in Two Italian Siblings. Parkinsons Dis, 2015. 2015: p. 546462.

56. Maraganore, D.M., et al., Collaborative analysis of alpha-synuclein gene promoter variability and Parkinson disease. JAMA, 2006. 296(6): p. 661-70.

57. Jo, E., et al., Defective membrane interactions of familial Parkinson's disease mutant A30P alpha-synuclein. J Mol Biol, 2002. 315(4): p. 799-807.

58. Fares, M.B., et al., The novel Parkinson's disease linked mutation G51D attenuates in vitro aggregation and membrane binding of alpha-synuclein, and enhances its secretion and nuclear localization in cells. Hum Mol Genet, 2014. 23(17): p. 4491-509.

59. Ghosh, D., et al., The newly discovered Parkinson's disease associated Finnish mutation (A53E) attenuates alpha-synuclein aggregation and membrane binding. Biochemistry, 2014. 53(41): p. 6419-21.

60. Ueda, K., et al., Molecular cloning of cDNA encoding an unrecognized component of amyloid in Alzheimer disease. Proc Natl Acad Sci U S A, 1993. 90(23): p. 11282-6.

61. Brown, D.R., Interactions between metals and alpha-synuclein--function or artefact? FEBS J, 2007. 274(15): p. 3766-74.

62. Nielsen, M.S., et al., Ca2+ binding to alpha-synuclein regulates ligand binding and oligomerization. J Biol Chem, 2001. 276(25): p. 22680-4.

63. Paik, S.R., et al., Copper(II)-induced self-oligomerization of alpha-synuclein. Biochem J, 1999. 340 ( Pt 3): p. 821-8. 
64. Wu, K.P., et al., Characterization of conformational and dynamic properties of natively unfolded human and mouse alpha-synuclein ensembles by NMR: implication for aggregation. J Mol Biol, 2008. 378(5): p. 1104-15.

65. Park, S.M., et al., Stress-induced aggregation profiles of GST-alpha-synuclein fusion proteins: role of the C-terminal acidic tail of alpha-synuclein in protein thermosolubility and stability. Biochemistry, 2002. 41(12): p. 4137-46.

66. Crowther, R.A., et al., Synthetic filaments assembled from C-terminally truncated alpha-synuclein. FEBS Lett, 1998. 436(3): p. 309-12.

67. Zhang, J., X. Li, and J.D. Li, The Roles of Post-translational Modifications on alphaSynuclein in the Pathogenesis of Parkinson's Diseases. Front Neurosci, 2019. 13: p. 381.

68. Okochi, M., et al., Constitutive phosphorylation of the Parkinson's disease associated alpha-synuclein. J Biol Chem, 2000. 275(1): p. 390-7.

69. Pronin, A.N., et al., Synucleins are a novel class of substrates for $G$ protein-coupled receptor kinases. J Biol Chem, 2000. 275(34): p. 26515-22.

70. Chen, L. and M.B. Feany, Alpha-synuclein phosphorylation controls neurotoxicity and inclusion formation in a Drosophila model of Parkinson disease. Nat Neurosci, 2005. 8(5): p. 657-63.

71. Ishii, A., et al., Casein kinase 2 is the major enzyme in brain that phosphorylates Ser129 of human alpha-synuclein: Implication for alpha-synucleinopathies. FEBS Lett, 2007. 581(24): p. 4711-7.

72. Paleologou, K.E., et al., Phosphorylation at 587 is enhanced in synucleinopathies, inhibits alpha-synuclein oligomerization, and influences synuclein-membrane interactions. J Neurosci, 2010. 30(9): p. 3184-98.

73. Kim, E.J., et al., Dyrk1A phosphorylates alpha-synuclein and enhances intracellular inclusion formation. J Biol Chem, 2006. 281(44): p. 33250-7.

74. Fujiwara, H., et al., alpha-Synuclein is phosphorylated in synucleinopathy lesions. Nat Cell Biol, 2002. 4(2): p. 160-4.

75. Hasegawa, M., et al., Phosphorylated alpha-synuclein is ubiquitinated in alphasynucleinopathy lesions. J Biol Chem, 2002. 277(50): p. 49071-6.

76. Anderson JP, W.D., Goldstein JM, de Laat R, Banducci K, Caccavello RJ, Barbour R, Huang J, Kling K, Lee M, Diep L, Keim PS, Shen X, Chataway T, Schlossmacher MG, Seubert P, Schenk D, Sinha S, Gai WP, Chilcote TJ., Phosphorylation of Ser-129 is the dominant pathological modification of alpha-synuclein in familial and sporadic Lewy body disease. J Biol Chem., 2006. 281(40): p. 29739-52.

77. Weinreb, P.H., et al., NACP, a protein implicated in Alzheimer's disease and learning, is natively unfolded. Biochemistry, 1996. 35(43): p. 13709-15.

78. Fauvet, B., et al., alpha-Synuclein in central nervous system and from erythrocytes, mammalian cells, and Escherichia coli exists predominantly as disordered monomer. J Biol Chem, 2012. 287(19): p. 15345-64.

79. Burre, J., et al., Properties of native brain alpha-synuclein. Nature, 2013. 498(7453): p. E4-6; discussion E6-7.

80. Bussell, R., Jr., T.F. Ramlall, and D. Eliezer, Helix periodicity, topology, and dynamics of membrane-associated alpha-synuclein. Protein Sci, 2005. 14(4): p. 862-72.

81. Davidson, W.S., et al., Stabilization of alpha-synuclein secondary structure upon binding to synthetic membranes. J Biol Chem, 1998. 273(16): p. 9443-9.

82. Eliezer, D., et al., Conformational properties of alpha-synuclein in its free and lipidassociated states. J Mol Biol, 2001. 307(4): p. 1061-73. 
83. Perrin, R.J., et al., Interaction of human alpha-Synuclein and Parkinson's disease variants with phospholipids. Structural analysis using site-directed mutagenesis. J Biol Chem, 2000. 275(44): p. 34393-8.

84. Bartels, T., J.G. Choi, and D.J. Selkoe, alpha-Synuclein occurs physiologically as a helically folded tetramer that resists aggregation. Nature, 2011. 477(7362): p. 107-10.

85. Wang, W., et al., A soluble alpha-synuclein construct forms a dynamic tetramer. Proc Natl Acad Sci U S A, 2011. 108(43): p. 17797-802.

86. Theillet, F.X., et al., Structural disorder of monomeric alpha-synuclein persists in mammalian cells. Nature, 2016. 530(7588): p. 45-50.

87. Burre, J., M. Sharma, and T.C. Sudhof, alpha-Synuclein assembles into higher-order multimers upon membrane binding to promote SNARE complex formation. Proc Natl Acad Sci U S A, 2014. 111(40): p. E4274-83.

88. Wang, L., et al., alpha-synuclein multimers cluster synaptic vesicles and attenuate recycling. Curr Biol, 2014. 24(19): p. 2319-26.

89. Conway, K.A., et al., Acceleration of oligomerization, not fibrillization, is a shared property of both alpha-synuclein mutations linked to early-onset Parkinson's disease: implications for pathogenesis and therapy. Proc Natl Acad Sci U S A, 2000. 97(2): p. 571-6.

90. El-Agnaf, O.M., et al., Aggregates from mutant and wild-type alpha-synuclein proteins and NAC peptide induce apoptotic cell death in human neuroblastoma cells by formation of beta-sheet and amyloid-like filaments. FEBS Lett, 1998. 440(1-2): p. 71-5.

91. Ding, T.T., et al., Annular alpha-synuclein protofibrils are produced when spherical protofibrils are incubated in solution or bound to brain-derived membranes. Biochemistry, 2002. 41(32): p. 10209-17.

92. Lashuel, H.A., et al., Alpha-synuclein, especially the Parkinson's disease-associated mutants, forms pore-like annular and tubular protofibrils. J Mol Biol, 2002. 322(5): p. 1089-102.

93. Uversky, V.N., Neuropathology, biochemistry, and biophysics of alpha-synuclein aggregation. J Neurochem, 2007. 103(1): p. 17-37.

94. Wong, Y.C. and D. Krainc, alpha-synuclein toxicity in neurodegeneration: mechanism and therapeutic strategies. Nat Med, 2017. 23(2): p. 1-13.

95. Alzheimer, A., et al., An English translation of Alzheimer's 1907 paper, "Uber eine eigenartige Erkankung der Hirnrinde". Clin Anat, 1995. 8(6): p. 429-31.

96. Alzheimer, A., Über eigenartige Krankheitsfälle des späteren Alters. Zeitschrift für die gesamte Neurologie und Psychiatrie, 1911. 4(1): p. 356.

97. Alzheimer, A., Uber eine eigenartige Erkrankung der Hirnrinde. Zentralbl. Nervenh. Psych., 1907. 18: p. 177-179.

98. Fischer, O., Miliare Nekrose mit drusigen Wucherungen der Neurofibrillen, eine regelmässige Veränderung der Hirnrinde bei seniler Demenz. Monatsschrift Psychiatr. Neurol., 1907. 22:361-72.

99. Goedert, M., Oskar Fischer and the study of dementia. Brain, 2009. 132(Pt 4): p. 110211.

100. Grundke-Iqbal, I., et al., Abnormal phosphorylation of the microtubule-associated protein tau (tau) in Alzheimer cytoskeletal pathology. Proc Natl Acad Sci U S A, 1986. 83(13): p. 4913-7.

101. Grundke-lqbal, I., et al., Microtubule-associated protein tau. A component of Alzheimer paired helical filaments. J Biol Chem, 1986. 261(13): p. 6084-9. 
102. Kidd, M., Paired helical filaments in electron microscopy of Alzheimer's disease. Nature, 1963. 197: p. 192-3.

103. Arendt, T., J.T. Stieler, and M. Holzer, Tau and tauopathies. Brain Res Bull, 2016. 126(Pt 3): p. 238-292.

104. Gotz, J., G. Halliday, and R.M. Nisbet, Molecular Pathogenesis of the Tauopathies. Annu Rev Pathol, 2019. 14: p. 239-261.

105. Mackenzie, I.R. and M. Neumann, Molecular neuropathology of frontotemporal dementia: insights into disease mechanisms from postmortem studies. J Neurochem, 2016. 138 Suppl 1: p. 54-70.

106. Odawara, T., et al., Investigation of tau-2 positive microglia-like cells in the subcortical nuclei of human neurodegenerative disorders. Neurosci Lett, 1995. 192(3): p. 145-8.

107. Komori, T., Tau-positive glial inclusions in progressive supranuclear palsy, corticobasal degeneration and Pick's disease. Brain Pathol, 1999. 9(4): p. 663-79.

108. Zanier, E.R., et al., Induction of a transmissible tau pathology by traumatic brain injury. Brain, 2018. 141(9): p. 2685-2699.

109. Wallin, A.K., et al., CSF biomarkers predict a more malignant outcome in Alzheimer disease. Neurology, 2010. 74(19): p. 1531-7.

110. Kester, M.I., et al., CSF biomarkers predict rate of cognitive decline in Alzheimer disease. Neurology, 2009. 73(17): p. 1353-8.

111. Nelson, P.T., et al., Correlation of Alzheimer disease neuropathologic changes with cognitive status: a review of the literature. J Neuropathol Exp Neurol, 2012. 71(5): p. 362-81.

112. Scholl, M., et al., PET Imaging of Tau Deposition in the Aging Human Brain. Neuron, 2016. 89(5): p. 971-982.

113. Goedert, M., et al., Multiple isoforms of human microtubule-associated protein tau: sequences and localization in neurofibrillary tangles of Alzheimer's disease. Neuron, 1989. 3(4): p. 519-26.

114. Guo, T., W. Noble, and D.P. Hanger, Roles of tau protein in health and disease. Acta Neuropathol, 2017. 133(5): p. 665-704.

115. Simic, G., A. Diana, and P.R. Hof, Phosphorylation pattern of tau associated with distinct changes of the growth cone cytoskeleton. Prog Mol Subcell Biol, 2003. 32: p. 33-48.

116. Taniguchi-Watanabe, S., et al., Biochemical classification of tauopathies by immunoblot, protein sequence and mass spectrometric analyses of sarkosyl-insoluble and trypsin-resistant tau. Acta Neuropathol, 2016. 131(2): p. 267-280.

117. Arai, T., et al., Identification of amino-terminally cleaved tau fragments that distinguish progressive supranuclear palsy from corticobasal degeneration. Ann Neurol, 2004. 55(1): p. 72-9.

118. Zhong, Q., et al., Tau isoform composition influences rate and extent of filament formation. J Biol Chem, 2012. 287(24): p. 20711-9.

119. von Bergen, M., et al., Assembly of tau protein into Alzheimer paired helical filaments depends on a local sequence motif ((306)VQIVYK(311)) forming beta structure. Proc Natl Acad Sci U S A, 2000. 97(10): p. 5129-34.

120. Silva, M.C. and S.J. Haggarty, Tauopathies: Deciphering Disease Mechanisms to Develop Effective Therapies. Int J Mol Sci, 2020. 21(23). 
121. Matsumoto, S.E., et al., The twenty-four KDa C-terminal tau fragment increases with aging in tauopathy mice: implications of prion-like properties. Hum Mol Genet, 2015. 24(22): p. 6403-16.

122. Paholikova, K., et al., $\mathrm{N}$-terminal truncation of microtubule associated protein tau dysregulates its cellular localization. J Alzheimers Dis, 2015. 43(3): p. 915-26.

123. Gauthier-Kemper, A., et al., The frontotemporal dementia mutation R406W blocks tau's interaction with the membrane in an annexin A2-dependent manner. J Cell Biol, 2011. 192(4): p. 647-61.

124. Morris, M., et al., The many faces of tau. Neuron, 2011. 70(3): p. 410-26.

125. Wang, X., et al., The proline-rich domain and the microtubule binding domain of protein tau acting as RNA binding domains. Protein Pept Lett, 2006. 13(7): p. 679-85.

126. Quintanilla, R.A., et al., Caspase-cleaved tau expression induces mitochondrial dysfunction in immortalized cortical neurons: implications for the pathogenesis of Alzheimer disease. J Biol Chem, 2009. 284(28): p. 18754-66.

127. Goedert, M. and M.G. Spillantini, Propagation of Tau aggregates. Mol Brain, 2017. 10(1): p. 18.

128. Abraha, A., et al., C-terminal inhibition of tau assembly in vitro and in Alzheimer's disease. J Cell Sci, 2000. 113 Pt 21: p. 3737-45.

129. Flores-Rodriguez, P., et al., The relationship between truncation and phosphorylation at the C-terminus of tau protein in the paired helical filaments of Alzheimer's disease. Front Neurosci, 2015. 9: p. 33.

130. Kosik, K.S. and E.A. Finch, MAP2 and tau segregate into dendritic and axonal domains after the elaboration of morphologically distinct neurites: an immunocytochemical study of cultured rat cerebrum. J Neurosci, 1987. 7(10): p. 3142-53.

131. Camero, S., et al., Tau protein provides DNA with thermodynamic and structural features which are similar to those found in histone-DNA complex. J Alzheimers Dis, 2014. 39(3): p. 649-60.

132. Li, X.C., et al., Human wild-type full-length tau accumulation disrupts mitochondrial dynamics and the functions via increasing mitofusins. Sci Rep, 2016. 6: p. 24756.

133. Ittner, L.M., et al., Dendritic function of tau mediates amyloid-beta toxicity in Alzheimer's disease mouse models. Cell, 2010. 142(3): p. 387-97.

134. Kimura, T., et al., Microtubule-associated protein tau is essential for long-term depression in the hippocampus. Philos Trans R Soc Lond B Biol Sci, 2014. 369(1633): p. 20130144.

135. Brandt, R., J. Leger, and G. Lee, Interaction of tau with the neural plasma membrane mediated by tau's amino-terminal projection domain. J Cell Biol, 1995. 131(5): p. 132740.

136. Aamodt, E.J. and R.C. Williams, Jr., Microtubule-associated proteins connect microtubules and neurofilaments in vitro. Biochemistry, 1984. 23(25): p. 6023-31.

137. Bertrand, A., et al., Non-invasive, in vivo monitoring of neuronal transport impairment in a mouse model of tauopathy using MEMRI. Neuroimage, 2013. 64: p. 693-702.

138. Ebneth, A., et al., Overexpression of tau protein inhibits kinesin-dependent trafficking of vesicles, mitochondria, and endoplasmic reticulum: implications for Alzheimer's disease. J Cell Biol, 1998. 143(3): p. 777-94.

139. Zhang, B., et al., Retarded axonal transport of R406W mutant tau in transgenic mice with a neurodegenerative tauopathy. J Neurosci, 2004. 24(19): p. 4657-67. 
140. Dou, F., et al., Chaperones increase association of tau protein with microtubules. Proc Natl Acad Sci U S A, 2003. 100(2): p. 721-6.

141. Georgieva, E.R., et al., Tau binds to lipid membrane surfaces via short amphipathic helices located in its microtubule-binding repeats. Biophys J, 2014. 107(6): p. 1441-52.

142. Klein, C., et al., Process outgrowth of oligodendrocytes is promoted by interaction of fyn kinase with the cytoskeletal protein tau. J Neurosci, 2002. 22(3): p. 698-707.

143. Cardona-Gomez, G.P., et al., Estrogen dissociates Tau and alpha-amino-3-hydroxy-5methylisoxazole-4-propionic acid receptor subunit in postischemic hippocampus. Neuroreport, 2006. 17(12): p. 1337-41.

144. Gomez-Ramos, A., et al., Extracellular tau promotes intracellular calcium increase through M1 and M3 muscarinic receptors in neuronal cells. Mol Cell Neurosci, 2008. 37(4): p. 673-81.

145. Pallas-Bazarra, N., et al., Novel function of Tau in regulating the effects of external stimuli on adult hippocampal neurogenesis. EMBO J, 2016. 35(13): p. 1417-36.

146. Dalby, N.O., et al., Altered function of hippocampal CA1 pyramidal neurons in the rTg4510 mouse model of tauopathy. J Alzheimers Dis, 2014. 40(2): p. 429-42.

147. Kopeikina, K.J., et al., Tau causes synapse loss without disrupting calcium homeostasis in the rTg4510 model of tauopathy. PLoS One, 2013. 8(11): p. e80834.

148. Hoover, B.R., et al., Tau mislocalization to dendritic spines mediates synaptic dysfunction independently of neurodegeneration. Neuron, 2010. 68(6): p. 1067-81.

149. Roberson, E.D., et al., Reducing endogenous tau ameliorates amyloid beta-induced deficits in an Alzheimer's disease mouse model. Science, 2007. 316(5825): p. 750-4.

150. DeVos, S.L., et al., Antisense reduction of tau in adult mice protects against seizures. J Neurosci, 2013. 33(31): p. 12887-97.

151. Hatch, R.J., et al., Hyperphosphorylated tau causes reduced hippocampal CA1 excitability by relocating the axon initial segment. Acta Neuropathol, 2017. 133(5): p. 717-730.

152. Crimins, J.L., A.B. Rocher, and J.I. Luebke, Electrophysiological changes precede morphological changes to frontal cortical pyramidal neurons in the rTg4510 mouse model of progressive tauopathy. Acta Neuropathol, 2012. 124(6): p. 777-95.

153. Goedert, M. and M.G. Spillantini, Tau gene mutations and neurodegeneration. Biochem Soc Symp, 2001(67): p. 59-71.

154. Hutton, M., et al., Association of missense and $5^{\prime}$-splice-site mutations in tau with the inherited dementia FTDP-17. Nature, 1998. 393(6686): p. 702-5.

155. Spillantini, M.G., et al., Mutation in the tau gene in familial multiple system tauopathy with presenile dementia. Proc Natl Acad Sci U S A, 1998. 95(13): p. 7737-41.

156. Ghetti, B., et al., Invited review: Frontotemporal dementia caused by microtubuleassociated protein tau gene (MAPT) mutations: a chameleon for neuropathology and neuroimaging. Neuropathol Appl Neurobiol, 2015. 41(1): p. 24-46.

157. Sposito, T., et al., Developmental regulation of tau splicing is disrupted in stem cellderived neurons from frontotemporal dementia patients with the $10+16$ splice-site mutation in MAPT. Hum Mol Genet, 2015. 24(18): p. 5260-9.

158. Liu, F. and C.X. Gong, Tau exon 10 alternative splicing and tauopathies. Mol Neurodegener, 2008. 3: p. 8.

159. Cleveland, D.W., S.Y. Hwo, and M.W. Kirschner, Physical and chemical properties of purified tau factor and the role of tau in microtubule assembly. J Mol Biol, 1977. 116(2): p. 227-47. 
160. Avila, J., et al., Tau Structures. Front Aging Neurosci, 2016. 8: p. 262.

161. Polanco, J.C., et al., Amyloid-beta and tau complexity - towards improved biomarkers and targeted therapies. Nat Rev Neurol, 2018. 14(1): p. 22-39.

162. Fitzpatrick, A.W.P., et al., Cryo-EM structures of tau filaments from Alzheimer's disease. Nature, 2017. 547(7662): p. 185-190.

163. Morris, M., et al., Tau post-translational modifications in wild-type and human amyloid precursor protein transgenic mice. Nat Neurosci, 2015. 18(8): p. 1183-9.

164. Li, C. and J. Gotz, Tau-based therapies in neurodegeneration: opportunities and challenges. Nat Rev Drug Discov, 2017. 16(12): p. 863-883.

165. Goedert, M. and R. Jakes, Expression of separate isoforms of human tau protein: correlation with the tau pattern in brain and effects on tubulin polymerization. EMBO J, 1990. 9(13): p. 4225-30.

166. Ksiezak-Reding, H., W.K. Liu, and S.H. Yen, Phosphate analysis and dephosphorylation of modified tau associated with paired helical filaments. Brain Res, 1992. 597(2): p. 209-19.

167. Hanger, D.P., B.H. Anderton, and W. Noble, Tau phosphorylation: the therapeutic challenge for neurodegenerative disease. Trends Mol Med, 2009. 15(3): p. 112-9.

168. Zoghbi, H.Y. and H.T. Orr, Glutamine repeats and neurodegeneration. Annu Rev Neurosci, 2000. 23: p. 217-47.

169. Lieberman, A.P., V.G. Shakkottai, and R.L. Albin, Polyglutamine Repeats in Neurodegenerative Diseases. Annu Rev Pathol, 2019. 14: p. 1-27.

170. MacDonald, M.E., et al., A novel gene containing a trinucleotide repeat that is expanded and unstable on Huntington's disease chromosomes. Cell, 1993. 72(6): p. 971-983.

171. Koide, R., et al., Unstable expansion of CAG repeat in hereditary dentatorubralpallidoluysian atrophy (DRPLA). Nat Genet, 1994. 6(1): p. 9-13.

172. Nagafuchi, S., et al., Dentatorubral and pallidoluysian atrophy expansion of an unstable CAG trinucleotide on chromosome 12p. Nat Genet, 1994. 6(1): p. 14-8.

173. David, G., et al., Molecular and clinical correlations in autosomal dominant cerebellar ataxia with progressive macular dystrophy (SCA7). Hum Mol Genet, 1998. 7(2): p. 16570.

174. Kawaguchi, Y., et al., CAG expansions in a novel gene for Machado-Joseph disease at chromosome 14q32.1. Nat Genet, 1994. 8(3): p. 221-8.

175. Nakamura, K., et al., SCA17, a novel autosomal dominant cerebellar ataxia caused by an expanded polyglutamine in TATA-binding protein. Hum Mol Genet, 2001. 10(14): p. 1441-8.

176. Nechiporuk, A., et al., Genetic mapping of the spinocerebellar ataxia type 2 gene on human chromosome 12. Neurology, 1996. 46(6): p. 1731-5.

177. Orr, H.T., et al., Expansion of an unstable trinucleotide CAG repeat in spinocerebellar ataxia type 1. Nat Genet, 1993. 4(3): p. 221-6.

178. Zhuchenko, O., et al., Autosomal dominant cerebellar ataxia (SCA6) associated with small polyglutamine expansions in the alpha $1 A$-voltage-dependent calcium channel. Nat Genet, 1997. 15(1): p. 62-9.

179. La Spada, A.R., et al., Androgen receptor gene mutations in X-linked spinal and bulbar muscular atrophy. Nature, 1991. 352(6330): p. 77-9.

180. Gatchel, J.R. and H.Y. Zoghbi, Diseases of unstable repeat expansion: mechanisms and common principles. Nat Rev Genet, 2005. 6(10): p. 743-55. 
181. Silva, A., A.V. de Almeida, and S. Macedo-Ribeiro, Polyglutamine expansion diseases: More than simple repeats. J Struct Biol, 2018. 201(2): p. 139-154.

182. Havel, L.S., S. Li, and X.J. Li, Nuclear accumulation of polyglutamine disease proteins and neuropathology. Mol Brain, 2009. 2: p. 21.

183. Paulson, H.L., et al., Intranuclear inclusions of expanded polyglutamine protein in spinocerebellar ataxia type 3. Neuron, 1997. 19(2): p. 333-44.

184. Wexler, A., E.J. Wild, and S.J. Tabrizi, George Huntington: a legacy of inquiry, empathy and hope. Brain, 2016. 139(Pt 8): p. 2326-33.

185. Tabrizi, S.J., et al., Predictors of phenotypic progression and disease onset in premanifest and early-stage Huntington's disease in the TRACK-HD study: analysis of 36-month observational data. Lancet Neurol, 2013. 12(7): p. 637-49.

186. Bates, G.P., et al., Huntington disease. Nat Rev Dis Primers, 2015. 1: p. 15005.

187. Vonsattel, J.P., Huntington disease models and human neuropathology: similarities and differences. Acta Neuropathol, 2008. 115(1): p. 55-69.

188. Reiner, A., I. Dragatsis, and P. Dietrich, Genetics and neuropathology of Huntington's disease. Int Rev Neurobiol, 2011. 98: p. 325-72.

189. Rosas, H.D., et al., Cerebral cortex and the clinical expression of Huntington's disease: complexity and heterogeneity. Brain, 2008. 131(Pt 4): p. 1057-68.

190. Han, I., et al., Differential vulnerability of neurons in Huntington's disease: the role of cell type-specific features. J Neurochem, 2010. 113(5): p. 1073-91.

191. Tang, C. and A. Feigin, Monitoring Huntington's disease progression through preclinical and early stages. Neurodegener Dis Manag, 2012. 2(4): p. 421-435.

192. Ha, A.D. and V.S. Fung, Huntington's disease. Curr Opin Neurol, 2012. 25(4): p. 491-8.

193. Zheng, Z. and M.I. Diamond, Huntington disease and the huntingtin protein. Prog Mol Biol Transl Sci, 2012. 107: p. 189-214.

194. McColgan, P. and S.J. Tabrizi, Huntington's disease: a clinical review. Eur J Neurol, 2018. 25(1): p. 24-34.

195. Carroll, J.B., et al., Treating the whole body in Huntington's disease. Lancet Neurol, 2015. 14(11): p. 1135-42.

196. A novel gene containing a trinucleotide repeat that is expanded and unstable on Huntington's disease chromosomes. The Huntington's Disease Collaborative Research Group. Cell, 1993. 72(6): p. 971-83.

197. Squitieri, F. and J. Jankovic, Huntington's disease: how intermediate are intermediate repeat lengths? Mov Disord, 2012. 27(14): p. 1714-7.

198. Telenius, H., et al., Somatic and gonadal mosaicism of the Huntington disease gene CAG repeat in brain and sperm. Nat Genet, 1994. 6(4): p. 409-14.

199. Bates, G., Huntingtin aggregation and toxicity in Huntington's disease. Lancet, 2003. 361(9369): p. 1642-4.

200. Gafni, J., et al., Caspase-6 activity in a BACHD mouse modulates steady-state levels of mutant huntingtin protein but is not necessary for production of a 586 amino acid proteolytic fragment. J Neurosci, 2012. 32(22): p. 7454-65.

201. Goldberg, Y.P., et al., Cleavage of huntingtin by apopain, a proapoptotic cysteine protease, is modulated by the polyglutamine tract. Nat Genet, 1996. 13(4): p. 442-9.

202. Kim, Y.J., et al., Caspase 3-cleaved $\mathrm{N}$-terminal fragments of wild-type and mutant huntingtin are present in normal and Huntington's disease brains, associate with membranes, and undergo calpain-dependent proteolysis. Proc Natl Acad Sci U S A, 2001. 98(22): p. 12784-9. 
203. Miller, J.P., et al., Matrix metalloproteinases are modifiers of huntingtin proteolysis and toxicity in Huntington's disease. Neuron, 2010. 67(2): p. 199-212.

204. Saudou, F. and S. Humbert, The Biology of Huntingtin. Neuron, 2016. 89(5): p. 910-26.

205. Atwal, R.S., et al., Huntingtin has a membrane association signal that can modulate huntingtin aggregation, nuclear entry and toxicity. Hum Mol Genet, 2007. 16(21): p. 2600-15.

206. Rockabrand, E., et al., The first 17 amino acids of Huntingtin modulate its sub-cellular localization, aggregation and effects on calcium homeostasis. Hum Mol Genet, 2007. 16(1): p. 61-77.

207. Maiuri, T., et al., The huntingtin N17 domain is a multifunctional CRM1 and Randependent nuclear and cilial export signal. Hum Mol Genet, 2013. 22(7): p. 1383-94.

208. Steffan, J.S., et al., SUMO modification of Huntingtin and Huntington's disease pathology. Science, 2004. 304(5667): p. 100-4.

209. Thompson, L.M., et al., IKK phosphorylates Huntingtin and targets it for degradation by the proteasome and lysosome. J Cell Biol, 2009. 187(7): p. 1083-99.

210. Neveklovska, M., et al., Deletion of the huntingtin proline-rich region does not significantly affect normal huntingtin function in mice. J Huntingtons Dis, 2012. 1(1): p. 71-87.

211. Palidwor, G.A., et al., Detection of alpha-rod protein repeats using a neural network and application to huntingtin. PLoS Comput Biol, 2009. 5(3): p. e1000304.

212. Andrade, M.A. and P. Bork, HEAT repeats in the Huntington's disease protein. Nat Genet, 1995. 11(2): p. 115-6.

213. Ratovitski, T., et al., Post-Translational Modifications (PTMs), Identified on Endogenous Huntingtin, Cluster within Proteolytic Domains between HEAT Repeats. J Proteome Res, 2017. 16(8): p. 2692-2708.

214. Xia, J., et al., Huntingtin contains a highly conserved nuclear export signal. Hum Mol Genet, 2003. 12(12): p. 1393-403.

215. Bessert, D.A., et al., The identification of a functional nuclear localization signal in the Huntington disease protein. Brain Res Mol Brain Res, 1995. 33(1): p. 165-73.

216. Zheng, Z., et al., An N-terminal nuclear export signal regulates trafficking and aggregation of Huntingtin (Htt) protein exon 1. J Biol Chem, 2013. 288(9): p. 6063-71.

217. Nasir, J., et al., Targeted disruption of the Huntington's disease gene results in embryonic lethality and behavioral and morphological changes in heterozygotes. Cell, 1995. 81(5): p. 811-23.

218. Zeitlin, S., et al., Increased apoptosis and early embryonic lethality in mice nullizygous for the Huntington's disease gene homologue. Nat Genet, 1995. 11(2): p. 155-63.

219. Schulte, J. and J.T. Littleton, The biological function of the Huntingtin protein and its relevance to Huntington's Disease pathology. Curr Trends Neurol, 2011. 5: p. 65-78.

220. Sassone, J., et al., Huntington's disease: the current state of research with peripheral tissues. Exp Neurol, 2009. 219(2): p. 385-97.

221. Ehrnhoefer, D.E., L. Sutton, and M.R. Hayden, Small changes, big impact: posttranslational modifications and function of huntingtin in Huntington disease. Neuroscientist, 2011. 17(5): p. 475-92.

222. Hodges, A., et al., Regional and cellular gene expression changes in human Huntington's disease brain. Hum Mol Genet, 2006. 15(6): p. 965-77.

223. Tabrizi, S.J., et al., Huntington disease: new insights into molecular pathogenesis and therapeutic opportunities. Nat Rev Neurol, 2020. 16(10): p. 529-546. 
224. Difiglia, M., et al., Aggregation of huntingtin in neuronal intranuclear inclusions and dystrophic neurites in brain. Science, 1997. 277(5334): p. 1990-3.

225. Hoffner, G., M.L. Island, and P. Djian, Purification of neuronal inclusions of patients with Huntington's disease reveals a broad range of $N$-terminal fragments of expanded huntingtin and insoluble polymers. J Neurochem, 2005. 95(1): p. 125-36.

226. McGowan, D., et al., Amyloid-like inclusions in Huntington's disease. Neuroscience, 2000. 100(4): p. 677-680.

227. Huang, C.C., et al., Amyloid formation by mutant huntingtin: threshold, progressivity and recruitment of normal polyglutamine proteins. Somat Cell Mol Genet, 1998. 24(4): p. 217-33.

228. Hoffner, G. and P. Djian, Polyglutamine Aggregation in Huntington Disease: Does Structure Determine Toxicity? Mol Neurobiol, 2015. 52(3): p. 1297-1314.

229. Arrasate, M., et al., Inclusion body formation reduces levels of mutant huntingtin and the risk of neuronal death. Nature, 2004. 431(7010): p. 805-10.

230. Nucifora, L.G., et al., Identification of novel potentially toxic oligomers formed in vitro from mammalian-derived expanded huntingtin exon-1 protein. J Biol Chem, 2012. 287(19): p. 16017-28.

231. Nagai, Y., et al., A toxic monomeric conformer of the polyglutamine protein. Nat Struct Mol Biol, 2007. 14(4): p. 332-40.

232. Saudou, F., et al., Huntingtin acts in the nucleus to induce apoptosis but death does not correlate with the formation of intranuclear inclusions. Cell, 1998. 95(1): p. 55-66.

233. Slow, E.J., et al., Absence of behavioral abnormalities and neurodegeneration in vivo despite widespread neuronal huntingtin inclusions. Proc Natl Acad Sci U S A, 2005. 102(32): p. 11402-7.

234. Sathasivam, K., et al., Aberrant splicing of HTT generates the pathogenic exon 1 protein in Huntington disease. Proc Natl Acad Sci U S A, 2013. 110(6): p. 2366-70.

235. Sieradzan, K.A., et al., Huntington's disease intranuclear inclusions contain truncated, ubiquitinated huntingtin protein. Exp Neurol, 1999. 156(1): p. 92-9.

236. Consortium, H.D.i., Induced pluripotent stem cells from patients with Huntington's disease show CAG-repeat-expansion-associated phenotypes. Cell Stem Cell, 2012. 11(2): p. 264-78.

237. Prusiner, S.B., Novel proteinaceous infectious particles cause scrapie. Science, 1982. 216(4542): p. 136-44.

238. Aguzzi, A. and A.K.K. Lakkaraju, Cell Biology of Prions and Prionoids: A Status Report. Trends Cell Biol, 2016. 26(1): p. 40-51.

239. Prusiner, S.B., Molecular biology of prion diseases. Science, 1991. 252(5012): p. 151522.

240. Imran, M. and S. Mahmood, An overview of human prion diseases. Virol J, 2011. 8: p. 559.

241. Scialo, C., et al., Prion and Prion-Like Protein Strains: Deciphering the Molecular Basis of Heterogeneity in Neurodegeneration. Viruses, 2019. 11(3).

242. Parchi, P., et al., Classification of sporadic Creutzfeldt-Jakob disease based on molecular and phenotypic analysis of 300 subjects. Ann Neurol, 1999. 46(2): p. 22433.

243. DeArmond, S.J. and S.B. Prusiner, Etiology and pathogenesis of prion diseases. Am J Pathol, 1995. 146(4): p. 785-811. 
244. Takada, L.T. and M.D. Geschwind, Prion diseases. Semin Neurol, 2013. 33(4): p. 34856.

245. Mayor, S. and H. Riezman, Sorting GPI-anchored proteins. Nat Rev Mol Cell Biol, 2004. 5(2): p. 110-20.

246. Riek, R., et al., NMR structure of the mouse prion protein domain PrP(121-231). Nature, 1996. 382(6587): p. 180-2.

247. Shyng, S.L., J.E. Heuser, and D.A. Harris, A glycolipid-anchored prion protein is endocytosed via clathrin-coated pits. J Cell Biol, 1994. 125(6): p. 1239-50.

248. Linsenmeier, L., et al., Diverse functions of the prion protein - Does proteolytic processing hold the key? Biochim Biophys Acta Mol Cell Res, 2017. 1864(11 Pt B): p. 2128-2137.

249. Steele, A.D., et al., Prion protein (PrPc) positively regulates neural precursor proliferation during developmental and adult mammalian neurogenesis. Proc Natl Acad Sci U S A, 2006. 103(9): p. 3416-21.

250. Santuccione, A., et al., Prion protein recruits its neuronal receptor NCAM to lipid rafts to activate p59fyn and to enhance neurite outgrowth. J Cell Biol, 2005. 169(2): p. 34154.

251. Bremer, J., et al., Axonal prion protein is required for peripheral myelin maintenance. Nat Neurosci, 2010. 13(3): p. 310-8.

252. Mouillet-Richard, S., et al., Signal transduction through prion protein. Science, 2000. 289(5486): p. 1925-8.

253. Mallucci, G.R., et al., Post-natal knockout of prion protein alters hippocampal CA1 properties, but does not result in neurodegeneration. EMBO J, 2002. 21(3): p. 202-10.

254. Marin-Moreno, A., et al., Transmission and Replication of Prions. Prog Mol Biol Transl Sci, 2017. 150: p. 181-201.

255. Brandner, S., et al., Normal host prion protein (PrPC) is required for scrapie spread within the central nervous system. Proc Natl Acad Sci U S A, 1996. 93(23): p. 13148-51.

256. Huang, F.P. and G.G. MacPherson, Dendritic cells and oral transmission of prion diseases. Adv Drug Deliv Rev, 2004. 56(6): p. 901-13.

257. Bras, I.C. and T.F. Outeiro, Alpha-Synuclein: Mechanisms of Release and Pathology Progression in Synucleinopathies. Cells, 2021. 10(2).

258. Prusiner, S.B., Biology and genetics of prions causing neurodegeneration. Annu Rev Genet, 2013. 47: p. 601-23.

259. Sigurdson, C.J., J.C. Bartz, and M. Glatzel, Cellular and Molecular Mechanisms of Prion Disease. Annu Rev Pathol, 2019. 14: p. 497-516.

260. Apostol, M.I., et al., Atomic structures suggest determinants of transmission barriers in mammalian prion disease. Biochemistry, 2011. 50(13): p. 2456-63.

261. Sawaya, M.R., et al., Atomic structures of amyloid cross-beta spines reveal varied steric zippers. Nature, 2007. 447(7143): p. 453-7.

262. Kurt, T.D., et al., Prion transmission prevented by modifying the beta2-alpha2 loop structure of host PrPC. J Neurosci, 2014. 34(3): p. 1022-7.

263. Kurt, T.D., et al., Human prion protein sequence elements impede cross-species chronic wasting disease transmission. J Clin Invest, 2015. 125(4): p. 1485-96.

264. Yim, Y.I., et al., The multivesicular body is the major internal site of prion conversion. J Cell Sci, 2015. 128(7): p. 1434-43.

265. Brettschneider, J., et al., Spreading of pathology in neurodegenerative diseases: a focus on human studies. Nat Rev Neurosci, 2015. 16(2): p. 109-20. 
266. Arellano-Anaya, Z.E., et al., Prion strains are differentially released through the exosomal pathway. Cell Mol Life Sci, 2015. 72(6): p. 1185-96.

267. Vella, L.J., et al., Packaging of prions into exosomes is associated with a novel pathway of PrP processing. J Pathol, 2007. 211(5): p. 582-590.

268. Coleman, B.M., et al., Prion-infected cells regulate the release of exosomes with distinct ultrastructural features. FASEB J, 2012. 26(10): p. 4160-73.

269. Gousset, K., et al., Prions hijack tunnelling nanotubes for intercellular spread. Nat Cell Biol, 2009. 11(3): p. 328-36.

270. Zhu, S., et al., Prion aggregates transfer through tunneling nanotubes in endocytic vesicles. Prion, 2015. 9(2): p. 125-35.

271. Prusiner, S.B., Prions and neurodegenerative diseases. N Engl J Med, 1987. 317(25): p. 1571-81.

272. Scheckel, C. and A. Aguzzi, Prions, prionoids and protein misfolding disorders. Nat Rev Genet, 2018. 19(7): p. 405-418.

273. Shahnawaz, M., et al., Discriminating alpha-synuclein strains in Parkinson's disease and multiple system atrophy. Nature, 2020. 578(7794): p. 273-277.

274. Lau, A., et al., alpha-Synuclein strains target distinct brain regions and cell types. Nat Neurosci, 2020. 23(1): p. 21-31.

275. Peng, C., et al., Cellular milieu imparts distinct pathological alpha-synuclein strains in alpha-synucleinopathies. Nature, 2018. 557(7706): p. 558-563.

276. Peng, C., R.J. Gathagan, and V.M. Lee, Distinct alpha-Synuclein strains and implications for heterogeneity among alpha-Synucleinopathies. Neurobiol Dis, 2018. 109(Pt B): p. 209-218.

277. Kaufman, S.K., et al., Tau Prion Strains Dictate Patterns of Cell Pathology, Progression Rate, and Regional Vulnerability In Vivo. Neuron, 2016. 92(4): p. 796-812.

278. Sanders, D.W., et al., Distinct tau prion strains propagate in cells and mice and define different tauopathies. Neuron, 2014. 82(6): p. 1271-88.

279. Masnata, M. and F. Cicchetti, The Evidence for the Spread and Seeding Capacities of the Mutant Huntingtin Protein in in Vitro Systems and Their Therapeutic Implications. Front Neurosci, 2017. 11: p. 647.

280. Masnata, M., et al., Demonstration of prion-like properties of mutant huntingtin fibrils in both in vitro and in vivo paradigms. Acta Neuropathol, 2019. 137(6): p. 981-1001.

281. Tamguney, G. and A.D. Korczyn, A critical review of the prion hypothesis of human synucleinopathies. Cell Tissue Res, 2018. 373(1): p. 213-220.

282. Alpaugh, M. and F. Cicchetti, Huntington's disease: lessons from prion disorders. J Neurol, 2021.

283. Brunello, C.A., et al., Mechanisms of secretion and spreading of pathological tau protein. Cell Mol Life Sci, 2020. 77(9): p. 1721-1744.

284. Jucker, M. and L.C. Walker, Self-propagation of pathogenic protein aggregates in neurodegenerative diseases. Nature, 2013. 501(7465): p. 45-51.

285. Walker, L.C. and M. Jucker, Neurodegenerative diseases: expanding the prion concept. Annu Rev Neurosci, 2015. 38: p. 87-103.

286. Jucker, M. and L.C. Walker, Propagation and spread of pathogenic protein assemblies in neurodegenerative diseases. Nat Neurosci, 2018. 21(10): p. 1341-1349.

287. Iturria-Medina, Y. and A.C. Evans, On the central role of brain connectivity in neurodegenerative disease progression. Front Aging Neurosci, 2015. 7: p. 90. 
288. Lee, J.G., et al., Unconventional secretion of misfolded proteins promotes adaptation to proteasome dysfunction in mammalian cells. Nat Cell Biol, 2016. 18(7): p. 765-76.

289. Ariazi, J., et al., Tunneling Nanotubes and Gap Junctions-Their Role in Long-Range Intercellular Communication during Development, Health, and Disease Conditions. Front Mol Neurosci, 2017. 10: p. 333.

290. Victoria, G.S. and C. Zurzolo, The spread of prion-like proteins by lysosomes and tunneling nanotubes: Implications for neurodegenerative diseases. J Cell Biol, 2017. 216(9): p. 2633-2644.

291. Abounit, S. and C. Zurzolo, Wiring through tunneling nanotubes--from electrical signals to organelle transfer. J Cell Sci, 2012. 125(Pt 5): p. 1089-98.

292. Gerdes, H.H., A. Rustom, and X. Wang, Tunneling nanotubes, an emerging intercellular communication route in development. Mech Dev, 2013. 130(6-8): p. 381-7.

293. You, Y. and T. Ikezu, Emerging roles of extracellular vesicles in neurodegenerative disorders. Neurobiol Dis, 2019. 130: p. 104512.

294. Meldolesi, J., Exosomes and Ectosomes in Intercellular Communication. Curr Biol, 2018. 28(8): p. R435-R444.

295. van Niel, G., G. D'Angelo, and G. Raposo, Shedding light on the cell biology of extracellular vesicles. Nat Rev Mol Cell Biol, 2018. 19(4): p. 213-228.

296. Mathieu, M., et al., Specificities of secretion and uptake of exosomes and other extracellular vesicles for cell-to-cell communication. Nat Cell Biol, 2019. 21(1): p. 9-17.

297. Chiasserini, D., et al., Proteomic analysis of cerebrospinal fluid extracellular vesicles: a comprehensive dataset. J Proteomics, 2014. 106: p. 191-204.

298. Coleman, B.M. and A.F. Hill, Extracellular vesicles--Their role in the packaging and spread of misfolded proteins associated with neurodegenerative diseases. Semin Cell Dev Biol, 2015. 40: p. 89-96.

299. Choi, D.S., et al., Proteomics, transcriptomics and lipidomics of exosomes and ectosomes. Proteomics, 2013. 13(10-11): p. 1554-71.

300. Bellingham, S.A., et al., Exosomes: vehicles for the transfer of toxic proteins associated with neurodegenerative diseases? Front Physiol, 2012. 3: p. 124.

301. Thompson, A.G., et al., Extracellular vesicles in neurodegenerative disease pathogenesis to biomarkers. Nat Rev Neurol, 2016. 12(6): p. 346-57.

302. Thery, C., et al., Minimal information for studies of extracellular vesicles 2018 (MISEV2018): a position statement of the International Society for Extracellular Vesicles and update of the MISEV2014 guidelines. J Extracell Vesicles, 2018. 7(1): p. 1535750.

303. Cocucci, E. and J. Meldolesi, Ectosomes and exosomes: shedding the confusion between extracellular vesicles. Trends Cell Biol, 2015. 25(6): p. 364-72.

304. Choi, D.S., et al., The protein interaction network of extracellular vesicles derived from human colorectal cancer cells. J Proteome Res, 2012. 11(2): p. 1144-51.

305. Mathivanan, S., H. Ji, and R.J. Simpson, Exosomes: extracellular organelles important in intercellular communication. J Proteomics, 2010. 73(10): p. 1907-20.

306. Willms, E., et al., Cells release subpopulations of exosomes with distinct molecular and biological properties. Sci Rep, 2016. 6: p. 22519.

307. Sadallah, S., C. Eken, and J.A. Schifferli, Ectosomes as modulators of inflammation and immunity. Clin Exp Immunol, 2011. 163(1): p. 26-32. 
308. Stein, J.M. and J.P. Luzio, Ectocytosis caused by sublytic autologous complement attack on human neutrophils. The sorting of endogenous plasma-membrane proteins and lipids into shed vesicles. Biochem J, 1991. 274 ( Pt 2): p. 381-6.

309. Choi, D.S., et al., Proteomics of extracellular vesicles: Exosomes and ectosomes. Mass Spectrom Rev, 2015. 34(4): p. 474-90.

310. Kalra, H., G.P. Drummen, and S. Mathivanan, Focus on Extracellular Vesicles: Introducing the Next Small Big Thing. Int J Mol Sci, 2016. 17(2): p. 170.

311. Hall, G.F. and S. Saman, Death or secretion? The demise of a plausible assumption about CSF-tau in Alzheimer Disease? Commun Integr Biol, 2012. 5(6): p. 623-6.

312. Boukouris, S. and S. Mathivanan, Exosomes in bodily fluids are a highly stable resource of disease biomarkers. Proteomics Clin Appl, 2015. 9(3-4): p. 358-67.

313. Kanninen, K.M., et al., Exosomes as new diagnostic tools in CNS diseases. Biochim Biophys Acta, 2016. 1862(3): p. 403-10.

314. Stewart, T., et al., Cerebrospinal fluid alpha-synuclein predicts cognitive decline in Parkinson disease progression in the DATATOP cohort. Am J Pathol, 2014. 184(4): p. 966-975.

315. Ning, H., et al., Baseline concentration of misfolded alpha-synuclein aggregates in cerebrospinal fluid predicts risk of cognitive decline in Parkinson's disease. Neuropathol Appl Neurobiol, 2019. 45(4): p. 398-409.

316. Sun, R., et al., Changes in the Morphology, Number, and Pathological Protein Levels of Plasma Exosomes May Help Diagnose Alzheimer's Disease. J Alzheimers Dis, 2020. 73(3): p. 909-917.

317. Dujardin, S., et al., Ectosomes: a new mechanism for non-exosomal secretion of tau protein. PLoS One, 2014. 9(6): p. e100760.

318. Spitzer, P., et al., Microvesicles from cerebrospinal fluid of patients with Alzheimer's disease display reduced concentrations of tau and APP protein. Sci Rep, 2019. 9(1): p. 7089.

319. Kumar, A., et al., Challenges in Biomaterial-Based Drug Delivery Approach for the Treatment of Neurodegenerative Diseases: Opportunities for Extracellular Vesicles. Int J Mol Sci, 2020. 22(1).

320. Muhammad, S.A., Are extracellular vesicles new hope in clinical drug delivery for neurological disorders? Neurochem Int, 2021. 144: p. 104955.

321. Yu, Y., et al., The role of exosomal microRNAs in central nervous system diseases. Mol Cell Biochem, 2021. 476(5): p. 2111-2124.

322. Didiot, M.C., et al., Exosome-mediated Delivery of Hydrophobically Modified siRNA for Huntingtin mRNA Silencing. Mol Ther, 2016. 24(10): p. 1836-1847.

323. Yang, J., et al., Exosome-mediated delivery of antisense oligonucleotides targeting alpha-synuclein ameliorates the pathology in a mouse model of Parkinson's disease. Neurobiol Dis, 2021. 148: p. 105218.

324. Beatriz, M., R. Vilaca, and C. Lopes, Exosomes: Innocent Bystanders or Critical Culprits in Neurodegenerative Diseases. Front Cell Dev Biol, 2021. 9: p. 635104.

325. Kordower, J.H., et al., Robust graft survival and normalized dopaminergic innervation do not obligate recovery in a Parkinson disease patient. Ann Neurol, 2017. 81(1): p. 46-57.

326. Kurowska, Z., et al., Signs of degeneration in 12-22-year old grafts of mesencephalic dopamine neurons in patients with Parkinson's disease. J Parkinsons Dis, 2011. 1(1): p. 83-92. 
327. Li, J.Y., et al., Lewy bodies in grafted neurons in subjects with Parkinson's disease suggest host-to-graft disease propagation. Nat Med, 2008. 14(5): p. 501-3.

328. Li, J.Y., et al., Characterization of Lewy body pathology in 12- and 16-year-old intrastriatal mesencephalic grafts surviving in a patient with Parkinson's disease. Mov Disord, 2010. 25(8): p. 1091-6.

329. Li, W., et al., Extensive graft-derived dopaminergic innervation is maintained 24 years after transplantation in the degenerating parkinsonian brain. Proc Natl Acad Sci U S A, 2016. 113(23): p. 6544-9.

330. Lindvall, O., et al., Evidence for long-term survival and function of dopaminergic grafts in progressive Parkinson's disease. Ann Neurol, 1994. 35(2): p. 172-80.

331. Olanow, C.W., et al., A double-blind controlled trial of bilateral fetal nigral transplantation in Parkinson's disease. Ann Neurol, 2003. 54(3): p. 403-14.

332. Kordower, J.H., et al., Lewy body-like pathology in long-term embryonic nigral transplants in Parkinson's disease. Nat Med, 2008. 14(5): p. 504-6.

333. Kordower, J.H., et al., Transplanted dopaminergic neurons develop PD pathologic changes: a second case report. Mov Disord, 2008. 23(16): p. 2303-6.

334. Saito, Y., et al., Accumulation of phosphorylated alpha-synuclein in aging human brain. J Neuropathol Exp Neurol, 2003. 62(6): p. 644-54.

335. Braak, H., et al., Pathology associated with sporadic Parkinson's disease--where does it end? J Neural Transm Suppl, 2006(70): p. 89-97.

336. Shannon, K.M., et al., Alpha-synuclein in colonic submucosa in early untreated Parkinson's disease. Mov Disord, 2012. 27(6): p. 709-15.

337. Braak, H., et al., Gastric alpha-synuclein immunoreactive inclusions in Meissner's and Auerbach's plexuses in cases staged for Parkinson's disease-related brain pathology. Neurosci Lett, 2006. 396(1): p. 67-72.

338. Desplats, P., et al., Inclusion formation and neuronal cell death through neuron-toneuron transmission of alpha-synuclein. Proc Natl Acad Sci U S A, 2009. 106(31): p. 13010-5.

339. Hansen, C., et al., alpha-Synuclein propagates from mouse brain to grafted dopaminergic neurons and seeds aggregation in cultured human cells. J Clin Invest, 2011. 121(2): p. 715-25.

340. Luk, K.C., et al., Pathological alpha-synuclein transmission initiates Parkinson-like neurodegeneration in nontransgenic mice. Science, 2012. 338(6109): p. 949-53.

341. Luk, K.C., et al., Intracerebral inoculation of pathological alpha-synuclein initiates a rapidly progressive neurodegenerative alpha-synucleinopathy in mice. J Exp Med, 2012. 209(5): p. 975-86.

342. Mougenot, A.L., et al., Prion-like acceleration of a synucleinopathy in a transgenic mouse model. Neurobiol Aging, 2012. 33(9): p. 2225-8.

343. Masuda-Suzukake, M., et al., Pathological alpha-synuclein propagates through neural networks. Acta Neuropathol Commun, 2014. 2: p. 88.

344. Recasens, A., et al., Lewy body extracts from Parkinson disease brains trigger alphasynuclein pathology and neurodegeneration in mice and monkeys. Ann Neurol, 2014. 75(3): p. 351-62.

345. Rey, N.L., et al., Widespread transneuronal propagation of alpha-synucleinopathy triggered in olfactory bulb mimics prodromal Parkinson's disease. J Exp Med, 2016. 213(9): p. 1759-78. 
346. Sacino, A.N., et al., Intramuscular injection of alpha-synuclein induces CNS alphasynuclein pathology and a rapid-onset motor phenotype in transgenic mice. Proc Natl Acad Sci U S A, 2014. 111(29): p. 10732-7.

347. Peelaerts, W., et al., alpha-Synuclein strains cause distinct synucleinopathies after local and systemic administration. Nature, 2015. 522(7556): p. 340-4.

348. Ayers, J.I., et al., Robust Central Nervous System Pathology in Transgenic Mice following Peripheral Injection of alpha-Synuclein Fibrils. J Virol, 2017. 91(2).

349. Lee, H.J., S. Patel, and S.J. Lee, Intravesicular localization and exocytosis of alphasynuclein and its aggregates. J Neurosci, 2005. 25(25): p. 6016-24.

350. Sung, J.Y., et al., Proteolytic cleavage of extracellular secreted \{alpha\}-synuclein via matrix metalloproteinases. J Biol Chem, 2005. 280(26): p. 25216-24.

351. El-Agnaf, O.M., et al., Detection of oligomeric forms of alpha-synuclein protein in human plasma as a potential biomarker for Parkinson's disease. FASEB J, 2006. 20(3): p. 419-25.

352. Mollenhauer, B., et al., Direct quantification of CSF alpha-synuclein by ELISA and first cross-sectional study in patients with neurodegeneration. Exp Neurol, 2008. 213(2): p. 315-25.

353. El-Agnaf, O.M., et al., Alpha-synuclein implicated in Parkinson's disease is present in extracellular biological fluids, including human plasma. FASEB J, 2003. 17(13): p. 19457.

354. Dehay, B., et al., Alpha-synuclein propagation: New insights from animal models. Mov Disord, 2016. 31(2): p. 161-8.

355. Ye, Y., Regulation of protein homeostasis by unconventional protein secretion in mammalian cells. Semin Cell Dev Biol, 2018. 83: p. 29-35.

356. Brundin, P. and R. Melki, Prying into the Prion Hypothesis for Parkinson's Disease. J Neurosci, 2017. 37(41): p. 9808-9818.

357. Auluck, P.K., G. Caraveo, and S. Lindquist, alpha-Synuclein: membrane interactions and toxicity in Parkinson's disease. Annu Rev Cell Dev Biol, 2010. 26: p. 211-33.

358. Ahn, K.J., et al., Amino acid sequence motifs and mechanistic features of the membrane translocation of alpha-synuclein. J Neurochem, 2006. 97(1): p. 265-79.

359. Lee, H.J., et al., Assembly-dependent endocytosis and clearance of extracellular alphasynuclein. Int J Biochem Cell Biol, 2008. 40(9): p. 1835-49.

360. Paillusson, S., et al., Activity-dependent secretion of alpha-synuclein by enteric neurons. J Neurochem, 2013. 125(4): p. 512-7.

361. Jang, A., et al., Non-classical exocytosis of alpha-synuclein is sensitive to folding states and promoted under stress conditions. J Neurochem, 2010. 113(5): p. 1263-74.

362. Emmanouilidou, E., et al., Cell-produced alpha-synuclein is secreted in a calciumdependent manner by exosomes and impacts neuronal survival. J Neurosci, 2010. 30(20): p. 6838-51.

363. Alvarez-Erviti, L., et al., Lysosomal dysfunction increases exosome-mediated alphasynuclein release and transmission. Neurobiol Dis, 2011. 42(3): p. 360-7.

364. Danzer, K.M., et al., Exosomal cell-to-cell transmission of alpha synuclein oligomers. Mol Neurodegener, 2012. 7: p. 42.

365. Abounit, S., et al., Tunneling nanotubes spread fibrillar alpha-synuclein by intercellular trafficking of lysosomes. EMBO J, 2016. 35(19): p. 2120-2138. 
366. Dieriks, B.V., et al., alpha-synuclein transfer through tunneling nanotubes occurs in SHSY5Y cells and primary brain pericytes from Parkinson's disease patients. Sci Rep, 2017. 7: p. 42984.

367. Lashuel, H.A. and P.T. Lansbury, Jr., Are amyloid diseases caused by protein aggregates that mimic bacterial pore-forming toxins? Q Rev Biophys, 2006. 39(2): p. 167-201.

368. Yang, Y., et al., Secretory carrier membrane protein 5 is an autophagy inhibitor that promotes the secretion of alpha-synuclein via exosome. PLoS One, 2017. 12(7): p. e0180892.

369. Mao, X., et al., Pathological alpha-synuclein transmission initiated by binding lymphocyte-activation gene 3. Science, 2016. 353(6307).

370. van Rooijen, B.D., M.M. Claessens, and V. Subramaniam, Lipid bilayer disruption by oligomeric alpha-synuclein depends on bilayer charge and accessibility of the hydrophobic core. Biochim Biophys Acta, 2009. 1788(6): p. 1271-8.

371. Hoogerheide, D.P., et al., Mechanism of alpha-synuclein translocation through a VDAC nanopore revealed by energy landscape modeling of escape time distributions. Nanoscale, 2017. 9(1): p. 183-192.

372. Grey, M., et al., Acceleration of alpha-synuclein aggregation by exosomes. J Biol Chem, 2015. 290(5): p. 2969-82.

373. Stuendl, A., et al., Induction of alpha-synuclein aggregate formation by CSF exosomes from patients with Parkinson's disease and dementia with Lewy bodies. Brain, 2016. 139(Pt 2): p. 481-94.

374. Han, J., et al., The impact of obesity on brain iron levels and alpha-synuclein expression is regionally dependent. Nutr Neurosci, 2019. 22(5): p. 335-343.

375. Delenclos, M., et al., Investigation of Endocytic Pathways for the Internalization of Exosome-Associated Oligomeric Alpha-Synuclein. Front Neurosci, 2017. 11: p. 172.

376. Tomlinson, P.R., et al., Identification of distinct circulating exosomes in Parkinson's disease. Ann Clin Transl Neurol, 2015. 2(4): p. 353-61.

377. Sun, T., et al., Blood Exosomes Have Neuroprotective Effects in a Mouse Model of Parkinson's Disease. Oxid Med Cell Longev, 2020. 2020: p. 3807476.

378. Shi, M., et al., Plasma exosomal alpha-synuclein is likely CNS-derived and increased in Parkinson's disease. Acta Neuropathol, 2014. 128(5): p. 639-650.

379. Peelaerts, W., et al., a-Synuclein strains and seeding in Parkinson's disease, incidental Lewy body disease, dementia with Lewy bodies and multiple system atrophy: similarities and differences. Cell Tissue Res, 2018. 373(1): p. 195-212.

380. Jellinger, K.A., Formation and development of Lewy pathology: a critical update. J Neurol, 2009. 256 Suppl 3: p. 270-9.

381. Halliday, G., A. Lees, and M. Stern, Milestones in Parkinson's disease--clinical and pathologic features. Mov Disord, 2011. 26(6): p. 1015-21.

382. Surmeier, D.J., J.A. Obeso, and G.M. Halliday, Parkinson's Disease Is Not Simply a Prion Disorder. J Neurosci, 2017. 37(41): p. 9799-9807.

383. Jellinger, K.A., A critical evaluation of current staging of alpha-synuclein pathology in Lewy body disorders. Biochim Biophys Acta, 2009. 1792(7): p. 730-40.

384. Dijkstra, A.A., et al., Stage-dependent nigral neuronal loss in incidental Lewy body and Parkinson's disease. Mov Disord, 2014. 29(10): p. 1244-51.

385. Berg, D., et al., Time to redefine PD? Introductory statement of the MDS Task Force on the definition of Parkinson's disease. Mov Disord, 2014. 29(4): p. 454-62. 
386. Mendez, I., et al., Dopamine neurons implanted into people with Parkinson's disease survive without pathology for 14 years. Nat Med, 2008. 14(5): p. 507-9.

387. Hallett, P.J., et al., Long-term health of dopaminergic neuron transplants in Parkinson's disease patients. Cell Rep, 2014. 7(6): p. 1755-61.

388. Surmeier, D.J., J.A. Obeso, and G.M. Halliday, Selective neuronal vulnerability in Parkinson disease. Nat Rev Neurosci, 2017. 18(2): p. 101-113.

389. Clavaguera, F., et al., Brain homogenates from human tauopathies induce tau inclusions in mouse brain. Proc Natl Acad Sci U S A, 2013. 110(23): p. 9535-40.

390. Clavaguera, F., et al., Transmission and spreading of tauopathy in transgenic mouse brain. Nat Cell Biol, 2009. 11(7): p. 909-13.

391. Wu, J.W., et al., Small misfolded Tau species are internalized via bulk endocytosis and anterogradely and retrogradely transported in neurons. J Biol Chem, 2013. 288(3): p. 1856-70.

392. de Calignon, A., et al., Propagation of tau pathology in a model of early Alzheimer's disease. Neuron, 2012. 73(4): p. 685-97.

393. Liu, L., et al., Trans-synaptic spread of tau pathology in vivo. PLoS One, 2012. 7(2): p. e31302.

394. Iba, M., et al., Synthetic tau fibrils mediate transmission of neurofibrillary tangles in a transgenic mouse model of Alzheimer's-like tauopathy. J Neurosci, 2013. 33(3): p. 1024-37.

395. Espuny-Camacho, I., et al., Hallmarks of Alzheimer's Disease in Stem-Cell-Derived Human Neurons Transplanted into Mouse Brain. Neuron, 2017. 93(5): p. 1066-1081 e8.

396. Duka, V., et al., Identification of the sites of tau hyperphosphorylation and activation of tau kinases in synucleinopathies and Alzheimer's diseases. PLoS One, 2013. 8(9): p. e75025.

397. Nonaka, T., et al., Seeded aggregation and toxicity of \{alpha\}-synuclein and tau: cellular models of neurodegenerative diseases. J Biol Chem, 2010. 285(45): p. 3488598.

398. Aoyagi, A., et al., Abeta and tau prion-like activities decline with longevity in the Alzheimer's disease human brain. Sci Transl Med, 2019. 11(490).

399. Narasimhan, S., et al., Human tau pathology transmits glial tau aggregates in the absence of neuronal tau. J Exp Med, 2020. 217(2).

400. Lasagna-Reeves, C.A., et al., Alzheimer brain-derived tau oligomers propagate pathology from endogenous tau. Sci Rep, 2012. 2: p. 700.

401. Guo, J.L., et al., Unique pathological tau conformers from Alzheimer's brains transmit tau pathology in nontransgenic mice. J Exp Med, 2016. 213(12): p. 2635-2654.

402. Iba, M., et al., Tau pathology spread in PS19 tau transgenic mice following locus coeruleus (LC) injections of synthetic tau fibrils is determined by the $L C$ 's afferent and efferent connections. Acta Neuropathol, 2015. 130(3): p. 349-62.

403. Ahmed, Z., et al., A novel in vivo model of tau propagation with rapid and progressive neurofibrillary tangle pathology: the pattern of spread is determined by connectivity, not proximity. Acta Neuropathol, 2014. 127(5): p. 667-83.

404. Stancu, I.C., et al., Templated misfolding of Tau by prion-like seeding along neuronal connections impairs neuronal network function and associated behavioral outcomes in Tau transgenic mice. Acta Neuropathol, 2015. 129(6): p. 875-94. 
405. Holmes, B.B., et al., Heparan sulfate proteoglycans mediate internalization and propagation of specific proteopathic seeds. Proc Natl Acad Sci U S A, 2013. 110(33): p. E3138-47.

406. Clavaguera, F., et al., Peripheral administration of tau aggregates triggers intracerebral tauopathy in transgenic mice. Acta Neuropathol, 2014. 127(2): p. 299301.

407. Jack, C.R., Jr., et al., Tracking pathophysiological processes in Alzheimer's disease: an updated hypothetical model of dynamic biomarkers. Lancet Neurol, 2013. 12(2): p. 207-16.

408. Dubois, B., et al., Preclinical Alzheimer's disease: Definition, natural history, and diagnostic criteria. Alzheimers Dement, 2016. 12(3): p. 292-323.

409. Saper, C.B., B.H. Wainer, and D.C. German, Axonal and transneuronal transport in the transmission of neurological disease: potential role in system degenerations, including Alzheimer's disease. Neuroscience, 1987. 23(2): p. 389-98.

410. Mirbaha, H., et al., Inert and seed-competent tau monomers suggest structural origins of aggregation. Elife, 2018. 7.

411. Sharma, A.M., et al., Tau monomer encodes strains. Elife, 2018. 7.

412. Takashima, A., Tauopathies and tau oligomers. J Alzheimers Dis, 2013. 37(3): p. 565-8.

413. Pooler, A.M., et al., Physiological release of endogenous tau is stimulated by neuronal activity. EMBO Rep, 2013. 14(4): p. 389-94.

414. Yamada, K., et al., Neuronal activity regulates extracellular tau in vivo. J Exp Med, 2014. 211(3): p. 387-93.

415. Arai, H., et al., Tau in cerebrospinal fluid: a potential diagnostic marker in Alzheimer's disease. Ann Neurol, 1995. 38(4): p. 649-52.

416. Hampel, H., et al., Total and phosphorylated tau protein as biological markers of Alzheimer's disease. Exp Gerontol, 2010. 45(1): p. 30-40.

417. Dujardin, S., et al., Neuron-to-neuron wild-type Tau protein transfer through a transsynaptic mechanism: relevance to sporadic tauopathies. Acta Neuropathol Commun, 2014. 2: p. 14.

418. Calafate, S., et al., Synaptic Contacts Enhance Cell-to-Cell Tau Pathology Propagation. Cell Rep, 2015. 11(8): p. 1176-83.

419. Wu, J.W., et al., Neuronal activity enhances tau propagation and tau pathology in vivo. Nat Neurosci, 2016. 19(8): p. 1085-92.

420. Katsinelos, T., et al., Unconventional Secretion Mediates the Trans-cellular Spreading of Tau. Cell Rep, 2018. 23(7): p. 2039-2055.

421. Merezhko, M., et al., Secretion of Tau via an Unconventional Non-vesicular Mechanism. Cell Rep, 2018. 25(8): p. 2027-2035 e4.

422. Gauthier-Kemper, A., et al., Annexins A2 and A6 interact with the extreme $N$ terminus of tau and thereby contribute to tau's axonal localization. J Biol Chem, 2018. 293(21): p. 8065-8076.

423. Lasagna-Reeves, C.A., et al., The formation of tau pore-like structures is prevalent and cell specific: possible implications for the disease phenotypes. Acta Neuropathol Commun, 2014. 2: p. 56.

424. Patel, N., et al., Ion Channel Formation by Tau Protein: Implications for Alzheimer's Disease and Tauopathies. Biochemistry, 2015. 54(50): p. 7320-5.

425. Tardivel, M., et al., Tunneling nanotube (TNT)-mediated neuron-to neuron transfer of pathological Tau protein assemblies. Acta Neuropathol Commun, 2016. 4(1): p. 117. 
426. $\mathrm{Xu}, \mathrm{Y}$. , et al., DNAJC5 facilitates USP19-dependent unconventional secretion of misfolded cytosolic proteins. Cell Discov, 2018. 4: p. 11.

427. Baker, S., J.C. Polanco, and J. Gotz, Extracellular Vesicles Containing P301L Mutant Tau Accelerate Pathological Tau Phosphorylation and Oligomer Formation but Do Not Seed Mature Neurofibrillary Tangles in ALZ17 Mice. J Alzheimers Dis, 2016. 54(3): p. 12071217.

428. Saman, S., et al., Exosome-associated tau is secreted in tauopathy models and is selectively phosphorylated in cerebrospinal fluid in early Alzheimer disease. J Biol Chem, 2012. 287(6): p. 3842-9.

429. Ruan, Z., et al., Alzheimer's disease brain-derived extracellular vesicles spread tau pathology in interneurons. Brain, 2021. 144(1): p. 288-309.

430. Crotti, A., et al., BIN1 favors the spreading of Tau via extracellular vesicles. Sci Rep, 2019. 9(1): p. 9477.

431. Guix, F.X., et al., Detection of Aggregation-Competent Tau in Neuron-Derived Extracellular Vesicles. Int J Mol Sci, 2018. 19(3).

432. Wang, Y., et al., The release and trans-synaptic transmission of Tau via exosomes. Mol Neurodegener, 2017. 12(1): p. 5.

433. Aulston, B., et al., Extracellular Vesicles Isolated from Familial Alzheimer's Disease Neuronal Cultures Induce Aberrant Tau Phosphorylation in the Wild-Type Mouse Brain. J Alzheimers Dis, 2019. 72(2): p. 575-585.

434. Winston, C.N., et al., Neuronal Exosome-Derived Human Tau is Toxic to Recipient Mouse Neurons in vivo. J Alzheimers Dis, 2019. 67(2): p. 541-553.

435. Rauch, J.N., et al., Tau Internalization is Regulated by 6-O Sulfation on Heparan Sulfate Proteoglycans (HSPGs). Sci Rep, 2018. 8(1): p. 6382.

436. Cicchetti, F., et al., Mutant huntingtin is present in neuronal grafts in Huntington disease patients. Ann Neurol, 2014. 76(1): p. 31-42.

437. Yang, W., et al., Aggregated polyglutamine peptides delivered to nuclei are toxic to mammalian cells. Hum Mol Genet, 2002. 11(23): p. 2905-17.

438. Ren, P.H., et al., Cytoplasmic penetration and persistent infection of mammalian cells by polyglutamine aggregates. Nat Cell Biol, 2009. 11(2): p. 219-25.

439. Pecho-Vrieseling, E., et al., Transneuronal propagation of mutant huntingtin contributes to non-cell autonomous pathology in neurons. Nat Neurosci, 2014. 17(8): p. 1064-72.

440. Babcock, D.T. and B. Ganetzky, Transcellular spreading of huntingtin aggregates in the Drosophila brain. Proc Natl Acad Sci U S A, 2015. 112(39): p. E5427-33.

441. Jeon, I., et al., Human-to-mouse prion-like propagation of mutant huntingtin protein. Acta Neuropathol, 2016. 132(4): p. 577-92.

442. Costanzo, M., et al., Transfer of polyglutamine aggregates in neuronal cells occurs in tunneling nanotubes. J Cell Sci, 2013. 126(Pt 16): p. 3678-85.

443. Herrera, F., et al., Visualization of cell-to-cell transmission of mutant huntingtin oligomers. PLoS Curr, 2011. 3: p. RRN1210.

444. Pearce, M.M.P., et al., Prion-like transmission of neuronal huntingtin aggregates to phagocytic glia in the Drosophila brain. Nat Commun, 2015. 6: p. 6768.

445. Gosset, P., et al., Evidence for the spread of human-derived mutant huntingtin protein in mice and non-human primates. Neurobiol Dis, 2020. 141: p. 104941.

446. Rieux, M., et al., Shedding a new light on Huntington's disease: how blood can both propagate and ameliorate disease pathology. Mol Psychiatry, 2020. 
447. Ast, A., et al., mHTT Seeding Activity: A Marker of Disease Progression and Neurotoxicity in Models of Huntington's Disease. Mol Cell, 2018. 71(5): p. 675-688 e6.

448. Kim, D.K., et al., Cell-to-cell Transmission of Polyglutamine Aggregates in C. elegans. Exp Neurobiol, 2017. 26(6): p. 321-328.

449. Nekooki-Machida, Y., et al., Distinct conformations of in vitro and in vivo amyloids of huntingtin-exon1 show different cytotoxicity. Proc Natl Acad Sci U S A, 2009. 106(24): p. 9679-84.

450. Gutekunst, C.A., et al., Nuclear and neuropil aggregates in Huntington's disease: relationship to neuropathology. J Neurosci, 1999. 19(7): p. 2522-34.

451. Liu, Y., et al., Intergeneration CAG expansion in a Wuhan juvenile-onset Huntington disease family. Neurosci Bull, 2007. 23(4): p. 198-202.

452. Wild, E.J., et al., Quantification of mutant huntingtin protein in cerebrospinal fluid from Huntington's disease patients. J Clin Invest, 2015. 125(5): p. 1979-86.

453. Gupta, S., S. Jie, and D.W. Colby, Protein misfolding detected early in pathogenesis of transgenic mouse model of Huntington disease using amyloid seeding assay. J Biol Chem, 2012. 287(13): p. 9982-9989.

454. Tan, Z., et al., Huntington's disease cerebrospinal fluid seeds aggregation of mutant huntingtin. Mol Psychiatry, 2015. 20(11): p. 1286-93.

455. Trajkovic, K., H. Jeong, and D. Krainc, Mutant Huntingtin Is Secreted via a Late Endosomal/Lysosomal Unconventional Secretory Pathway. J Neurosci, 2017. 37(37): p. 9000-9012.

456. Zhang, X., et al., Potential Transfer of Polyglutamine and CAG-Repeat RNA in Extracellular Vesicles in Huntington's Disease: Background and Evaluation in Cell Culture. Cell Mol Neurobiol, 2016. 36(3): p. 459-70.

457. Deng, J., et al., Neurons Export Extracellular Vesicles Enriched in Cysteine String Protein and Misfolded Protein Cargo. Sci Rep, 2017. 7(1): p. 956.

458. Diaz-Hidalgo, L., et al., Transglutaminase type 2-dependent selective recruitment of proteins into exosomes under stressful cellular conditions. Biochim Biophys Acta, 2016. 1863(8): p. 2084-92.

459. Ananbeh, H., P. Vodicka, and H. Kupcova Skalnikova, Emerging Roles of Exosomes in Huntington's Disease. Int J Mol Sci, 2021. 22(8).

460. Denis, H.L., et al., Platelet-derived extracellular vesicles in Huntington's disease. J Neurol, 2018. 265(11): p. 2704-2712.

461. Hong, Y., et al., Mutant Huntingtin Inhibits alphaB-Crystallin Expression and Impairs Exosome Secretion from Astrocytes. J Neurosci, 2017. 37(39): p. 9550-9563.

462. Lee, M., et al., Exosomes from adipose-derived stem cells ameliorate phenotype of Huntington's disease in vitro model. Eur J Neurosci, 2016. 44(4): p. 2114-9.

463. Chen, S., F.A. Ferrone, and R. Wetzel, Huntington's disease age-of-onset linked to polyglutamine aggregation nucleation. Proc Natl Acad Sci U S A, 2002. 99(18): p. 11884-9.

464. Genetic Modifiers of Huntington's Disease Consortium. Electronic address, g.h.m.h.e. and C. Genetic Modifiers of Huntington's Disease, CAG Repeat Not Polyglutamine Length Determines Timing of Huntington's Disease Onset. Cell, 2019. 178(4): p. 887900 e14.

465. Morrison, P.J. and J. Benito-Leon, Neurologic features in intermediate allele carriers of Huntington disease. Neurology, 2016. 87(6): p. 556-7. 
466. Menendez-Gonzalez, M., et al., HTT gene intermediate alleles in neurodegeneration: evidence for association with Alzheimer's disease. Neurobiol Aging, 2019. 76: p. 215 e9-215 e14.

467. Kotler, S.A., et al., Probing initial transient oligomerization events facilitating Huntingtin fibril nucleation at atomic resolution by relaxation-based NMR. Proc Natl Acad Sci U S A, 2019. 116(9): p. 3562-3571.

468. Gusella, J.F., et al., A polymorphic DNA marker genetically linked to Huntington's disease. Nature, 1983. 306(5940): p. 234-8.

469. Yan, X., R.L. Uronen, and H.J. Huttunen, The interaction of alpha-synuclein and Tau: $A$ molecular conspiracy in neurodegeneration? Semin Cell Dev Biol, 2020. 99: p. 55-64.

470. Bloom, G.S., Amyloid-beta and tau: the trigger and bullet in Alzheimer disease pathogenesis. JAMA Neurol, 2014. 71(4): p. 505-8.

471. Li, X., et al., Novel diffusion barrier for axonal retention of Tau in neurons and its failure in neurodegeneration. EMBO J, 2011. 30(23): p. 4825-37.

472. Hampel, H., et al., Measurement of phosphorylated tau epitopes in the differential diagnosis of Alzheimer disease: a comparative cerebrospinal fluid study. Arch Gen Psychiatry, 2004. 61(1): p. 95-102.

473. Olsson, A., et al., Simultaneous measurement of beta-amyloid(1-42), total tau, and phosphorylated tau (Thr181) in cerebrospinal fluid by the XMAP technology. Clin Chem, 2005. 51(2): p. 336-45.

474. Robinson, J.L., et al., Neurodegenerative disease concomitant proteinopathies are prevalent, age-related and APOE4-associated. Brain, 2018. 141(7): p. 2181-2193.

475. Irwin, D.J., et al., Evaluation of potential infectivity of Alzheimer and Parkinson disease proteins in recipients of cadaver-derived human growth hormone. JAMA Neurol, 2013. 70(4): p. 462-8.

476. Hamilton, R.L., Lewy bodies in Alzheimer's disease: a neuropathological review of 145 cases using alpha-synuclein immunohistochemistry. Brain Pathol, 2000. 10(3): p. 37884.

477. Colom-Cadena, M., et al., Confluence of alpha-synuclein, tau, and beta-amyloid pathologies in dementia with Lewy bodies. J Neuropathol Exp Neurol, 2013. 72(12): p. 1203-12.

478. Lashley, T., et al., Cortical alpha-synuclein load is associated with amyloid-beta plaque burden in a subset of Parkinson's disease patients. Acta Neuropathol, 2008. 115(4): p. 417-25.

479. Pletnikova, O., et al., Abeta deposition is associated with enhanced cortical alphasynuclein lesions in Lewy body diseases. Neurobiol Aging, 2005. 26(8): p. 1183-92.

480. Badiola, N., et al., Tau enhances alpha-synuclein aggregation and toxicity in cellular models of synucleinopathy. PLoS One, 2011. 6(10): p. e26609.

481. Sengupta, U., et al., Pathological interface between oligomeric alpha-synuclein and tau in synucleinopathies. Biol Psychiatry, 2015. 78(10): p. 672-83.

482. Ishizawa, T., et al., Colocalization of tau and alpha-synuclein epitopes in Lewy bodies. J Neuropathol Exp Neurol, 2003. 62(4): p. 389-97.

483. Kotzbauer, P.T., et al., Fibrillization of alpha-synuclein and tau in familial Parkinson's disease caused by the A53T alpha-synuclein mutation. Exp Neurol, 2004. 187(2): p. 279-88.

484. Arima, K., et al., NACP/alpha-synuclein and tau constitute two distinctive subsets of filaments in the same neuronal inclusions in brains from a family of parkinsonism and 
dementia with Lewy bodies: double-immunolabeling fluorescence and electron microscopic studies. Acta Neuropathol, 2000. 100(2): p. 115-21.

485. Wills, J., et al., Elevated tauopathy and alpha-synuclein pathology in postmortem Parkinson's disease brains with and without dementia. Exp Neurol, 2010. 225(1): p. 210-8.

486. Haggerty, T., et al., Hyperphosphorylated Tau in an alpha-synuclein-overexpressing transgenic model of Parkinson's disease. Eur J Neurosci, 2011. 33(9): p. 1598-610.

487. Jensen, P.H., et al., alpha-synuclein binds to Tau and stimulates the protein kinase Acatalyzed tau phosphorylation of serine residues 262 and 356. J Biol Chem, 1999. 274(36): p. 25481-9.

488. Qureshi, H.Y. and H.K. Paudel, Parkinsonian neurotoxin 1-methyl-4-phenyl-1,2,3,6tetrahydropyridine (MPTP) and alpha-synuclein mutations promote Tau protein phosphorylation at Ser262 and destabilize microtubule cytoskeleton in vitro. J Biol Chem, 2011. 286(7): p. 5055-68.

489. Giasson, B.I., et al., Initiation and synergistic fibrillization of tau and alpha-synuclein. Science, 2003. 300(5619): p. 636-40.

490. Hadi, F., et al., alpha-synuclein abnormalities trigger focal tau pathology, spreading to various brain areas in Parkinson disease. J Neurochem, 2020.

491. Guo, J.L., et al., Distinct alpha-synuclein strains differentially promote tau inclusions in neurons. Cell, 2013. 154(1): p. 103-17.

492. Cisbani, G., et al., Presence of tau pathology within foetal neural allografts in patients with Huntington's and Parkinson's disease. Brain, 2017. 140(11): p. 2982-2992.

493. Ornelas, A.S., et al., Co-Existence of tau and alpha-synuclein pathology in fetal graft tissue at autopsy: A case report. Parkinsonism Relat Disord, 2020. 71: p. 36-39.

494. Fernandez-Nogales, M., et al., Huntington's disease is a four-repeat tauopathy with tau nuclear rods. Nat Med, 2014. 20(8): p. 881-5.

495. Gratuze, M., et al., Tau hyperphosphorylation and deregulation of calcineurin in mouse models of Huntington's disease. Hum Mol Genet, 2015. 24(1): p. 86-99.

496. Jeppesen, D.K., et al., Reassessment of Exosome Composition. Cell, 2019. 177(2): p. 428-445 e18.

497. Thery, C., et al., Isolation and characterization of exosomes from cell culture supernatants and biological fluids. Curr Protoc Cell Biol, 2006. Chapter 3: p. Unit 322.

498. Shelke, G.V., et al., Importance of exosome depletion protocols to eliminate functional and RNA-containing extracellular vesicles from fetal bovine serum. J Extracell Vesicles, 2014. 3.

499. Kowal, J., et al., Proteomic comparison defines novel markers to characterize heterogeneous populations of extracellular vesicle subtypes. Proc Natl Acad Sci U S A, 2016. 113(8): p. E968-77.

500. Zhang, $\mathrm{H}$., et al., Identification of distinct nanoparticles and subsets of extracellular vesicles by asymmetric flow field-flow fractionation. Nat Cell Biol, 2018. 20(3): p. 332343.

501. Vietri, M., M. Radulovic, and H. Stenmark, The many functions of ESCRTs. Nat Rev Mol Cell Biol, 2020. 21(1): p. 25-42.

502. Andreu, Z. and M. Yanez-Mo, Tetraspanins in extracellular vesicle formation and function. Front Immunol, 2014. 5: p. 442.

503. Keerthikumar, S., et al., ExoCarta: $A$ Web-Based Compendium of Exosomal Cargo. J Mol Biol, 2016. 428(4): p. 688-692. 
504. Kalra, H., et al., Vesiclepedia: a compendium for extracellular vesicles with continuous community annotation. PLoS Biol, 2012. 10(12): p. e1001450.

505. Muralidharan-Chari, V., et al., ARF6-regulated shedding of tumor cell-derived plasma membrane microvesicles. Curr Biol, 2009. 19(22): p. 1875-85.

506. Popa, S.J., S.E. Stewart, and K. Moreau, Unconventional secretion of annexins and galectins. Semin Cell Dev Biol, 2018. 83: p. 42-50.

507. Hoshino, A., et al., Tumour exosome integrins determine organotropic metastasis. Nature, 2015. 527(7578): p. 329-35.

508. Keerthikumar, S., et al., Proteogenomic analysis reveals exosomes are more oncogenic than ectosomes. Oncotarget, 2015. 6(17): p. 15375-96.

509. Taraboletti, G., et al., Shedding of the matrix metalloproteinases MMP-2, MMP-9, and MT1-MMP as membrane vesicle-associated components by endothelial cells. Am J Pathol, 2002. 160(2): p. 673-80.

510. Pathan, M., et al., Vesiclepedia 2019: a compendium of RNA, proteins, lipids and metabolites in extracellular vesicles. Nucleic Acids Res, 2019. 47(D1): p. D516-D519.

511. Szklarczyk, D., et al., STRING v11: protein-protein association networks with increased coverage, supporting functional discovery in genome-wide experimental datasets. Nucleic Acids Res, 2019. 47(D1): p. D607-D613.

512. von Mering, C., et al., STRING: known and predicted protein-protein associations, integrated and transferred across organisms. Nucleic Acids Res, 2005. 33(Database issue): p. D433-7.

513. Colombo, M., G. Raposo, and C. Thery, Biogenesis, secretion, and intercellular interactions of exosomes and other extracellular vesicles. Annu Rev Cell Dev Biol, 2014. 30: p. 255-89.

514. Clayton, A., et al., Analysis of antigen presenting cell derived exosomes, based on immuno-magnetic isolation and flow cytometry. J Immunol Methods, 2001. 247(1-2): p. 163-74.

515. Lotvall, J., et al., Minimal experimental requirements for definition of extracellular vesicles and their functions: a position statement from the International Society for Extracellular Vesicles. J Extracell Vesicles, 2014. 3: p. 26913.

516. Escrevente, C., et al., Interaction and uptake of exosomes by ovarian cancer cells. BMC Cancer, 2011. 11: p. 108.

517. Tario, J.D., Jr., et al., Optimized staining and proliferation modeling methods for cell division monitoring using cell tracking dyes. J Vis Exp, 2012(70): p. e4287.

518. Temchura, V.V., et al., Enhancement of immunostimulatory properties of exosomal vaccines by incorporation of fusion-competent $G$ protein of vesicular stomatitis virus. Vaccine, 2008. 26(29-30): p. 3662-72.

519. Suetsugu, A., et al., Imaging exosome transfer from breast cancer cells to stroma at metastatic sites in orthotopic nude-mouse models. Adv Drug Deliv Rev, 2013. 65(3): p. 383-90.

520. Nakase, I., et al., Active macropinocytosis induction by stimulation of epidermal growth factor receptor and oncogenic Ras expression potentiates cellular uptake efficacy of exosomes. Sci Rep, 2015. 5: p. 10300.

521. Roberts-Dalton, H.D., et al., Fluorescence labelling of extracellular vesicles using a novel thiol-based strategy for quantitative analysis of cellular delivery and intracellular traffic. Nanoscale, 2017. 9(36): p. 13693-13706. 
522. Takeda, S., et al., Neuronal uptake and propagation of a rare phosphorylated highmolecular-weight tau derived from Alzheimer's disease brain. Nat Commun, 2015. 6: p. 8490.

523. Frohlich, D., et al., Multifaceted effects of oligodendroglial exosomes on neurons: impact on neuronal firing rate, signal transduction and gene regulation. Philos Trans R Soc Lond B Biol Sci, 2014. 369(1652).

524. You, Y., et al., Activated human astrocyte-derived extracellular vesicles modulate neuronal uptake, differentiation and firing. J Extracell Vesicles, 2020. 9(1): p. 1706801.

525. Sharma, P., et al., Exosomes regulate neurogenesis and circuit assembly. Proc Natl Acad Sci U S A, 2019. 116(32): p. 16086-16094.

526. Antonucci, F., et al., Microvesicles released from microglia stimulate synaptic activity via enhanced sphingolipid metabolism. EMBO J, 2012. 31(5): p. 1231-40.

527. Sun, J.J., W. Kilb, and H.J. Luhmann, Self-organization of repetitive spike patterns in developing neuronal networks in vitro. Eur J Neurosci, 2010. 32(8): p. 1289-99.

528. Basso, M. and V. Bonetto, Extracellular Vesicles and a Novel Form of Communication in the Brain. Front Neurosci, 2016. 10: p. 127.

529. Paolicelli, R.C., G. Bergamini, and L. Rajendran, Cell-to-cell Communication by Extracellular Vesicles: Focus on Microglia. Neuroscience, 2019. 405: p. 148-157.

530. Hessvik, N.P. and A. Llorente, Current knowledge on exosome biogenesis and release. Cell Mol Life Sci, 2018. 75(2): p. 193-208.

531. Raposo, G. and W. Stoorvogel, Extracellular vesicles: exosomes, microvesicles, and friends. J Cell Biol, 2013. 200(4): p. 373-83.

532. Gangoda, L., et al., Extracellular vesicles including exosomes are mediators of signal transduction: are they protective or pathogenic? Proteomics, 2015. 15(2-3): p. 260-71.

533. Mulcahy, L.A., R.C. Pink, and D.R. Carter, Routes and mechanisms of extracellular vesicle uptake. J Extracell Vesicles, 2014. 3.

534. Szego, E.M., et al., Cytosolic Trapping of a Mitochondrial Heat Shock Protein Is an Early Pathological Event in Synucleinopathies. Cell Rep, 2019. 28(1): p. 65-77 e6.

535. Tiscornia, G., O. Singer, and I.M. Verma, Production and purification of lentiviral vectors. Nat Protoc, 2006. 1(1): p. 241-5.

536. Harris, J., et al., Non-plasma bonding of PDMS for inexpensive fabrication of microfluidic devices. J Vis Exp, 2007(9): p. 410.

537. Freundt, E.C., et al., Neuron-to-neuron transmission of alpha-synuclein fibrils through axonal transport. Ann Neurol, 2012. 72(4): p. 517-24.

538. Chiou, N.-T.A., K. Mark, Improved exosome isolation by sucrose gradient fractionation of ultracentrifuged crude exosome pellets. protocolexchange, 2016.

539. Shevchenko, A., et al., In-gel digestion for mass spectrometric characterization of proteins and proteomes. Nat Protoc, 2006. 1(6): p. 2856-60.

540. Cox, J. and M. Mann, MaxQuant enables high peptide identification rates, individualized p.p.b.-range mass accuracies and proteome-wide protein quantification. Nat Biotechnol, 2008. 26(12): p. 1367-72.

541. Tyanova, S., et al., The Perseus computational platform for comprehensive analysis of (prote)omics data. Nat Methods, 2016. 13(9): p. 731-40.

542. Wagenaar, D.A., J. Pine, and S.M. Potter, Effective parameters for stimulation of dissociated cultures using multi-electrode arrays. J Neurosci Methods, 2004. 138(1-2): p. 27-37. 
543. Khani, M.H. and T. Gollisch, Linear and nonlinear chromatic integration in the mouse retina. Nat Commun, 2021. 12(1): p. 1900.

544. Pachitariu, M., et al. Fast and accurate spike sorting of high-channel count probes with KiloSort. in NIPS Proceedings. 2016. Neural Information Systems Foundation, Inc.

545. Pachitariu, M., et al., Kilosort: realtime spike-sorting for extracellular electrophysiology with hundreds of channels. BioRxiv, 2016: p. 061481.

546. Schindelin, J., et al., Fiji: an open-source platform for biological-image analysis. Nat Methods, 2012. 9(7): p. 676-82.

547. Gan, L., et al., Converging pathways in neurodegeneration, from genetics to mechanisms. Nat Neurosci, 2018. 21(10): p. 1300-1309.

548. Braak, H. and K. Del Tredici, Potential Pathways of Abnormal Tau and alpha-Synuclein Dissemination in Sporadic Alzheimer's and Parkinson's Diseases. Cold Spring Harb Perspect Biol, 2016. 8(11).

549. Braak, H., et al., Staging of the intracerebral inclusion body pathology associated with idiopathic Parkinson's disease (preclinical and clinical stages). J Neurol, 2002. 249 Suppl 3: p. III/1-5.

550. Spires-Jones, T.L., J. Attems, and D.R. Thal, Interactions of pathological proteins in neurodegenerative diseases. Acta Neuropathol, 2017. 134(2): p. 187-205.

551. Attems, J., The multi-morbid old brain. Acta Neuropathol, 2017. 134(2): p. 169-170.

552. Outeiro, T.F., Emerging concepts in synucleinopathies. Acta Neuropathol, 2021. 141(4): p. 469-470.

553. Goedert, M., D.S. Eisenberg, and R.A. Crowther, Propagation of Tau Aggregates and Neurodegeneration. Annu Rev Neurosci, 2017. 40: p. 189-210.

554. Peng, C., J.Q. Trojanowski, and V.M. Lee, Protein transmission in neurodegenerative disease. Nat Rev Neurol, 2020. 16(4): p. 199-212.

555. Andrews, N.W., Regulated secretion of conventional lysosomes. Trends Cell Biol, 2000. 10(8): p. 316-21.

556. Ponpuak, M., et al., Secretory autophagy. Curr Opin Cell Biol, 2015. 35: p. 106-16.

557. Zhang, M. and R. Schekman, Cell biology. Unconventional secretion, unconventional solutions. Science, 2013. 340(6132): p. 559-61.

558. Lee, M.C., et al., Bi-directional protein transport between the ER and Golgi. Annu Rev Cell Dev Biol, 2004. 20: p. 87-123.

559. Hill, A.F., Extracellular Vesicles and Neurodegenerative Diseases. J Neurosci, 2019. 39(47): p. 9269-9273.

560. Abounit, S., et al., Tunneling nanotubes: A possible highway in the spreading of tau and other prion-like proteins in neurodegenerative diseases. Prion, 2016. 10(5): p. 344351.

561. Fowler, C.D., NeuroEVs: Characterizing Extracellular Vesicles Generated in the Neural Domain. J Neurosci, 2019. 39(47): p. 9262-9268.

562. Bras, I.C., et al., Ectosomes and exosomes are distinct proteomic entities that modulate spontaneous activity in neuronal cells. bioRxiv, 2021.

563. Jaunmuktane, Z. and S. Brandner, Invited Review: The role of prion-like mechanisms in neurodegenerative diseases. Neuropathol Appl Neurobiol, 2020. 46(6): p. 522-545.

564. Sanders, D.W., et al., Prions and Protein Assemblies that Convey Biological Information in Health and Disease. Neuron, 2016. 89(3): p. 433-48.

565. Soto, C. and S. Pritzkow, Protein misfolding, aggregation, and conformational strains in neurodegenerative diseases. Nat Neurosci, 2018. 21(10): p. 1332-1340. 
566. Budnik, V., C. Ruiz-Canada, and F. Wendler, Extracellular vesicles round off communication in the nervous system. Nat Rev Neurosci, 2016. 17(3): p. 160-72.

567. Franklin, H., B.E. Clarke, and R. Patani, Astrocytes and microglia in neurodegenerative diseases: Lessons from human in vitro models. Prog Neurobiol, 2021. 200: p. 101973.

568. Lively, S. and L.C. Schlichter, Microglia Responses to Pro-inflammatory Stimuli (LPS, IFNgamma+TNFalpha) and Reprogramming by Resolving Cytokines (IL-4, IL-10). Front Cell Neurosci, 2018. 12: p. 215.

569. Hung, S.Y., et al., Autophagy protects neuron from Abeta-induced cytotoxicity. Autophagy, 2009. 5(4): p. 502-10.

570. Brahic, M., et al., Axonal transport and secretion of fibrillar forms of alpha-synuclein, Abeta42 peptide and HTTExon 1. Acta Neuropathol, 2016. 131(4): p. 539-48.

571. Monsellier, E., L. Bousset, and R. Melki, alpha-Synuclein and huntingtin exon 1 amyloid fibrils bind laterally to the cellular membrane. Sci Rep, 2016. 6: p. 19180.

572. Ruiz-Arlandis, G., et al., Binding, internalization and fate of Huntingtin Exon1 fibrillar assemblies in mitotic and nonmitotic neuroblastoma cells. Neuropathol Appl Neurobiol, 2016. 42(2): p. 137-52.

573. Pieri, L., et al., Fibrillar alpha-synuclein and huntingtin exon 1 assemblies are toxic to the cells. Biophys J, 2012. 102(12): p. 2894-905.

574. Evans, L.D., et al., Extracellular Monomeric and Aggregated Tau Efficiently Enter Human Neurons through Overlapping but Distinct Pathways. Cell Rep, 2018. 22(13): p. 3612-3624.

575. Frost, B., R.L. Jacks, and M.I. Diamond, Propagation of tau misfolding from the outside to the inside of a cell. J Biol Chem, 2009. 284(19): p. 12845-52.

576. He, W.T., et al., Cytoplasmic Ubiquitin-Specific Protease 19 (USP19) Modulates Aggregation of Polyglutamine-Expanded Ataxin-3 and Huntingtin through the HSP9O Chaperone. PLoS One, 2016. 11(1): p. e0147515.

577. He, W.T., et al., HSP9O recognizes the $N$-terminus of huntingtin involved in regulation of huntingtin aggregation by USP19. Sci Rep, 2017. 7(1): p. 14797.

578. Tang, B.L., Unconventional Secretion and Intercellular Transfer of Mutant Huntingtin. Cells, 2018. 7(6).

579. Kwon, H.S. and S.H. Koh, Neuroinflammation in neurodegenerative disorders: the roles of microglia and astrocytes. Transl Neurodegener, 2020. 9(1): p. 42.

580. Palpagama, T.H., et al., The Role of Microglia and Astrocytes in Huntington's Disease. Front Mol Neurosci, 2019. 12: p. 258.

581. Velier, J., et al., Wild-type and mutant huntingtins function in vesicle trafficking in the secretory and endocytic pathways. Exp Neurol, 1998. 152(1): p. 34-40.

582. Volpicelli-Daley, L.A., et al., Exogenous alpha-synuclein fibrils induce Lewy body pathology leading to synaptic dysfunction and neuron death. Neuron, 2011. 72(1): p. 57-71.

583. Fortin, D.L., et al., Lipid rafts mediate the synaptic localization of alpha-synuclein. J Neurosci, 2004. 24(30): p. 6715-23.

584. Busch, D.J., et al., Acute increase of alpha-synuclein inhibits synaptic vesicle recycling evoked during intense stimulation. Mol Biol Cell, 2014. 25(24): p. 3926-41.

585. Hassink, G.C., et al., Exogenous alpha-synuclein hinders synaptic communication in cultured cortical primary rat neurons. PLoS One, 2018. 13(3): p. e0193763.

586. Shrivastava, A.N., et al., Differential Membrane Binding and Seeding of Distinct alphaSynuclein Fibrillar Polymorphs. Biophys J, 2020. 118(6): p. 1301-1320. 
587. Garcia-Agudo, L.F., et al., Genetically induced brain inflammation by Cnp deletion transiently benefits from microglia depletion. FASEB J, 2019. 33(7): p. 8634-8647.

588. Paiva, I., et al., A2A R-induced transcriptional deregulation in astrocytes: An in vitro study. Glia, 2019. 67(12): p. 2329-2342.

589. Kwan, W., et al., Mutant huntingtin impairs immune cell migration in Huntington disease. J Clin Invest, 2012. 122(12): p. 4737-47.

590. Slot, J.W. and H.J. Geuze, Cryosectioning and immunolabeling. Nat Protoc, 2007. 2(10): p. 2480-91.

591. Livak, K.J. and T.D. Schmittgen, Analysis of relative gene expression data using realtime quantitative PCR and the 2(-Delta Delta C(T)) Method. Methods, 2001. 25(4): p. 402-8.

592. Dominguez-Meijide, A., et al., Effects of pharmacological modulators of alphasynuclein and tau aggregation and internalization. Sci Rep, 2020. 10(1): p. 12827.

593. Ukmar-Godec, T., et al., Proteasomal degradation of the intrinsically disordered protein tau at single-residue resolution. Sci Adv, 2020. 6(30): p. eaba3916.

594. Reif, A., et al., Generation of Native, Untagged Huntingtin Exon1 Monomer and Fibrils Using a SUMO Fusion Strategy. J Vis Exp, 2018(136). 